\title{
Acknowledgment to Reviewers of the International Journal of Molecular Sciences in 2020
}

\author{
International Journal of Molecular Sciences Editorial Office
}

Citation: International Journal of Molecular Sciences Editorial Office. Acknowledgment to Reviewers of the International Journal of Molecular Sciences in 2020. Int. J. Mol. Sci. 2021, 22, 1123. https://doi.org/10.3390/ ijms22031123

Published: 23 January 2021

Publisher's Note: MDPI stays neutral with regard to jurisdictional claims in published maps and institutional affiliations.

\section{(i)}

Copyright: (c) 2021 by the author. Licensee MDPI, Basel, Switzerland. This article is an open access article distributed under the terms and conditions of the Creative Commons Attribution (CC BY) license (http://creativecommons.org/licenses /by/4.0/).

MDPI AG, St. Alban-Anlage 66, 4052 Basel, Switzerland

Peer review is the driving force of journal development, and reviewers are gatekeepers who ensure that the International Journal of Molecular Sciences maintains its standards for the high quality of its published papers. Thanks to the cooperation of our reviewers, in 2020, the median time to first decision was 15 days and the median time to publication was 33 days. The editors would like to express their sincere gratitude to the following reviewers for their precious time and dedication, regardless of whether the papers were finally published:

Aaron, Jean E.

Aaseth, Jan

Abad, Catalina

Abadía, Javier

Abalde-Cela, Sara

Abashev, George G.

Abate, Giulia

Abba, Mohammed L.

Abballe, Annalisa

Abbate, Loredana

Abbina, Srinivas

Abd El Hakim, Yasmina

Abdallah, Ali

Abdallah, Mohamed F.

Abdelalim, Essam M.

Abdelhafiz, Ahmed $\mathrm{H}$

Abdelkarim, Hazem

Abdellatif, Ahmed

Abdelmohsen, Usama Ramadan

Abdul-Muneer, P. M.

Abe, Masahiro

Abe, Tatsuya

Abedi Ardekani, Behnoush

Abedi, Tayebeh

Abend, Michael

Aberg, Christoffer

Abete Goñi, Itziar

Abeykoon, Jithma

Abia, Akebe Luther King

Abicht, Angela

Abinaya, Manivannan

Abkhalimov, Evgeny V.
Abo-Ismail, Mohammed

Abondio, Paolo

Aboubakar, Frank

Aboudehen, Karam

Abraham, Dietmar

Abraham, Nader

Abrahams, Jan Pieter

Abramyan, John

Ábrányi-Balogh, Péter

Abubakr, Neamat Hassan

Abu-Reidah, Ibrahim M.

Abu-Romman, Saeid

Abutarboush, Rania

Accardi, Luisa

Accardo, Angelo

Acedo Nunez, Maria Del Pilar

Acharya, Arpan

Acharya, Ghanashyam

Achim, Cristina

Achiron, Asaf

Acosta-Jurado, Sebastián

Acuña-Castroviejo, Darío

Adachi, Hiroaki

Adachi, Naoki

Adachi, Shunsuke

Ádám, Attila L.

Adam, Gerhard

Adamakis, Ioannis-Dimosthenis

Adamcakova-Dodd, Andrea

Adamczyk, Agata

Adamek, Jakub

Adamopoulos, Panagiotis 
Adams, Bryn L.

Adams, James

Adams, Volker

Adamska, Agnieszka

Adawy, Alaa

Adegbola, Raphael

Adekoya, Timothy O.

Adell, Teresa

Ådén, Jörgen

Adey, Deborah

Adhikari, Manish

Adhikari, Neeta

Adhikary, Amitava

Adil, Syed Farooq

Adina, Turcu-Stiolica

Adkar-Purushothama, Charith Raj

Adney, Danielle

Adolf-Bryfogle, Jared

Adrover, Alessandra

Adt, Isabelle

Adur, Malavika

Advani, Vivek

Afanassieff, Marielle

Afantitis, Antreas

Afolayan, Adeleye J.

Afonso, Clélia Neves

Agarwal, Gaurav

Agarwal, Poonam

Agatemor, Christian

Ageitos, Jose Manuel

Aggarwal, Bharat B.

Aggeli, Ioanna-Katerina

Aggrey, Samuel E.

Agić, Dejan

Agirre, Jon

Agnelli, Luca

Agnieszka, Gizak

Agostino, Mark

Agoulnik, Alexander

Agrawal, Sandeep K.

Aguennouz, M.

Aguilera-Aguirre, Leopoldo

Aguirre, Aitor

Agusa, Tetsuro

Agustí-Brisach, Carlos

Ahmad, Aamir

Ahmad, Ali

Ahmad, Asrar

Ahmad, Fahim

Ahmad, Ibrar

Ahmad, Shakil
Ahmed, Aisha Siddiqah

Ahmed, Atif Ali

Ahmed, Chulbul

Ahmed, Hafiz

Ahmed, Mukhtar

Ahmed, Nuzhat

Ahmed, Shafiq U

Ahmed, Zubair

Ahn, Chi Bum

Ahn, Dong-Kuk

Ahn, Eun Young

Ahsan, Md. Ariful

Aiba, Takeshi

Aiba, Yuichiro

Aidinis, Vassilis

Aiello, Francesca

Aimi, Junko

Aine, Mattias

Airenne, Kari

Aires, Inês Dinis

Aisa, Maria Cristina

Aissat, Abdel

Aitken, Karen S.

Ajduk, Anna

Ajjan, Ramzi

Akahoshi, Mitsuteru

Akam, Elizabeth C

Akhgari, Amir

Akhtar, Yasmin

Akimov, Sergey A.

Akitsu, Takashiro

Akiyama, Masashi

Akk, Gustav

Akopian, Armen

Akula, Shaw

Al Bari, Md. Abdullah

Al Rihani, Sweilem

Alaimo, Alessandro

Alain, Tommy

Alam, Mobashwer

Alam, Perwez

Alam, Shafiul

Alamillo, Josefa Muñoz

Alansary, Dalia

Al-Aubaidy, Hayder

Alavi, Saman

Alavi, Seyed Ebrahim

Albano, Emanuele

Albarracín, Sonia L.

Albeck, John

Albericio, Fernando 
Albert, Mareike

Albertolle, Matthew

Albrecht, Randy

Albrechtsen, Nicolai Jacob Wewer

Alcalde, Mireia

Alcántara-Ortigoza, Miguel Angel

Alcaro, Stefano

Alcolea, Veronica

Alcover, Andres

Aldabe, Rafael

Aldámiz-Echevarría, Luis

Alder, Janet

Aldieri, Elisabetta

Aldred, Nicholas

Alduina, Rosa

Al-Dujaili, Emad

Alecsandru, Diana M.

Aleffi, Michele

Alejandro, Santiago

Aleksandrovych, Veronika

Alekseev, Alexey

Aleksova, Aneta

Alessenko, Alice V.

Alessio, Nicola

Aleti, Gajender

Aleu, Josefina

Alewine, Christine

Alexander, Dorothea

Alexander, Francis Borgio J.

Alexander, H. Denis

Alexander, Mathew

Alexander, Rebecca

Alexandrescu, Sanda

Alexandrov, Oleg S.

Alexov, Emil

Alfano, Daniela

Alfano, Massimo

Alfarouk, Khalid Omer

Al-Fatimi, Mohamed

Alfei, Silvana

Alfieri, Maria Laura

Alfirevic, Ana

Al-Ghadban, Sara

Al-Haj-Zen, Ayman

Alhakamy, Nabil

Alhenc-Gélas, Francois

Al-Hilal, Taslim Ahmed

Ali, Akhtar

Ali, Ali

Ali, Hayssam M.

Ali, Tahir
Aliaño González, María José

Alicja, Macko-Podgorni

Aliev, Gjumrakch

Aliki, Xanthopoulou

Alina, Wiszniewska

Alique, Matilde

Alizargar, Javad

Aljabali, Alaa A A

Alkalaeva, Elena

Allan, Alison

Allegra, Mario

Allegrucci, Cinzia

Allen, Bruce G.

Allen-Petersen, Brittany

Allijn, Iris E

Allikmets, Rando L.

Allison, Lizabeth

Allison, Simon

Allison, W. Ted

Allmang, Christine

Alloza, Iraide

Allu, Prasanna K.R.

Almahli, Hadia

Almansa, María Soledad

Almasan, Alex

Almasri, Maher

Almeida, Luciana Oliveira

Almeida, Maria Rosário

Almeida, Pedro

Almeida, Vitor Hugo

Alméras, Tancrede

Almerico, Anna Maria

Al-Mrabeh, Ahmad

Al-Mubarak, Bashayer R.

Al-Mutairi, Nawaf

Aloman, Costica

Alonge, Salvatore

Alonso, Juan Carlos

Alonso, M. Beatriz Durán

Alonso, Sergio

Alpaugh, Katherine

AlQuraishi, Mohammed

Alsaab, Hashem

Alsadeq, Ameera

Alshareef, Abdulraheem

Alsina, Berta

Altamirano-Bustamante, Myriam M.

Altamura, Gennaro

Altmann, Brigitte

Altmann, Friedrich

Altmeppen, Hermann 
Altomonte, Jennifer

Altucci, Lucia

Álvarez Martín, Javier

Alvarez, Ana I.

Alvarez, Clara

Alvarez, Guzmán

Alvarez, Juan B.

Alvarez, Martina

Álvarez-González, Carlos Alfonso

Álvarez-Mercado, Ana Isabel

Alvarez-Pizarro, Juan Carlos

Álvarez-Sánchez, Nuria

Álvaro, Tomás

Alves E Silva, Thiago Luiz

Alves Lopes, João Pedro

Alves, C. Henrique

Alves, Celso

Alves, Joana

Alves, Marco

Alves, Nuno

Alves, Sandra

Alves, Vinicius

Alyamani, Mohammad

Alyass, Akram

Alzubaidi, Laith

Amabilino, David

Amadio, Marialaura

Amadio, Susanna

Amador-Noguez, Daniel

Amantini, Consuelo

Amaradhi, Radhika

Amaral, Alexandra

Amaral, Ana Claudia

Amaral, Olga

Amaral, Ronaldo

Amarelli, Cristiano

Amarilla, Alberto A.

Amaro, Francisco

Amasheh, Salah

Amata, Emanuele

Amato, Antonella

Amato, Felice

Ambrose, Chris

Ambrosini, Grazia

Amélie, Saunier

Ameln, Anne Klotzsche-von

Ameloot, Marcel

Amenabar, Maximiliano

Amendola, Roberto

Amengual, Jaume

Ames, James B
Amiel, Catherine

Amiel, Eyal

Amillis, Sotiris

Amils, Ricardo

Amin, Kawa

Amin, Muhammad Junaid

Amin, Nirav $\mathrm{H}$.

Amin, Rajesh

Amini, Hajar

Aminin, Dmitry

AMIRAL, Jean

Amiri, Achour

Amirkhani, Masoume

Amit, Moran

Amital, Howard

Amiya, Eisuke

Amler, Evzen

Ammendola, Serena

Amoaku, Winfried M.

Amodeo, Pietro

Amores, Marco

Amorim, Christiani

Amoroso, Rosa

Amoutzias, Grigoris

Ampofo, Emmanuel

Amrani, Abdelaziz

Amuzescu, Bogdan

An, Seong Soo A.

An, Seung

An, Tae Kyu

An, Wonsuk

An, Yu

Ana María, Rojo López

Anamthathmakula, Prashanth

Anand, Swati

Anandhan, Annadurai

Anastasi, Giuseppe Pio

Anastasio, Noelle

Anathy, Vikas

Anbarasu, Kumaraswamy

Ancel, Julien

Anchordoquy, Tom

Ancuceanu, Robert

Anderegg, Ulf

Andersen, Ethan J.

Andersen, Thomas Levin

Anderson, Charles

Anderson, Greg M.

Anderssion, Leif

Anderton, Ryan

Andia, Isabel 
Andicsov'a Eckstein, Anita

Andl, Thomas

Andón, Fernando Torres

András, Ibolya

Andre, Helder

Andreadou, Ioanna

Andreasson, Erik

Andrée, Birgit

Andreev, Dmitriy Nikolaevich

Andreev, Dmitry E

Andreev, Konstantin

André-Grégoire, Gwennan

Andreoli, Roberta

Andres-Mach, Marta

Andressen, Kjetil

Andreu, Vicente

Andreuzzi, Eva

Andrew, Tedder

Andrews, Allison M.

Andrews, Rachel N

Andric, Silvana

Andriessen, Anneke

Andrieux, Geoffroy

Andrukhov, Oleh

Andrzej, Nowicki

Aneja, Ritu

Aneskievich, Brian J.

Anestopoulos, Ioannis

Anfinogenova, Yana J.

Ang, Kok Siong

Ang, Zhiwei

Angastiniotis, Michael

Angelastro, James

Angeles-Shim, Rosalyn B.

Angeletti, Mauro

Angelici, Maria Cristina

Angelico, Giuseppe

Angelini, Francesco

Angelino, Donato

Angelis, Karel J.

Angelova, Assia L.

Angelucci, Cristiana

Angelucci, Emanuele

Anger, Martin

Angioni, Maria Maddalena

Angius, Fabrizio

Anglada-Huguet, Marta

Aniello, Francesco

Anis, Eman

Anisimova, Irina

Anita, Boratkó
Anitas, Eugen

Anna, Chlebowska-Śmigiel

Anna, Lange-Consiglio

Annaratone, Laura

Annese, Vito

Annesley, Sarah

Annibale, Paolo

Annibalini, Giosuè

Anraku, Makoto

Ansani, Lucia

Ansorena, Eduardo

Antal, Diana

Antal, László

Anticoli, Simona

Antinozzi, Cristina

Antipova, Veronica

Antognelli, Cinzia

Antoine, Marie-Hélène

Anton, Lennikov

Antonela Antoniu, Sabina

Antonelli, Alessandro

Antonello E Spinelli, Antonello E

Antonelou, Marianna $\mathrm{H}$.

Antonio Malfa, Giuseppe

Antonio, Crovace

Antonioli, Luca

Antonkiewicz, Jacek

Antonogeorgos, George

Antony, Jane

Antony, Marie Lue

Antosiewicz, Anti-oxidationJędrzej

Antunes, Henedina

Anver, Shajahan

Anwar, Muhammad Ayaz

Anyfantakis, Dimitrios I.

Aoe, Tomohiko

Aoki, Kosuke

Aoyama, Koji

Apel, Christian

Apetrei, Constantin

Apicella, Antonio

Apostolopoulos, Vasso

Apostolova, Elena

Appavu, Rajagopal

Appels, Rudi

Aprile, Alessio

Aprile, Francesco A.

Aprotosoaie, Ana Clara

Apte, Udayan

Arai, Toshiro

Araki, Manabu 
Araki, Toshiyuki

Aran, Gemma

Aranda, Carlos J.

Aránega, Amelia

Arango Duque, Guillermo

Araniciu, Cătălin

Arano, Yasushi

Arasanz Esteban, Hugo

Arata, Toshiaki

Aratani, Yasuaki

Araújo, Inês M.

Araujo, Ricardo

Archer, Edward

Archibong, Anthony

Arcidiacono, Biagio

Arciello, Angela

Arczewska, Katarzyna

Ardalan, Maryam

Ardévol, Anna

Ardi-Pastores, Veronica

Arechederra, María

Arena, Alessandro

Arenas, Jesús

Arenas, Miguel

Arend, Rebecca C.

Arevalo, Juan Carlos

Argentati, Chiara

Argentiero, Antonella

Argento, Sergio

Argueta, Donovan A.

Ariani, Alarico

Arias Mediano, José Luis

Arias-Santiago, Salvador

Aricò, Eleonora

Arif, Abul

Arif, Tasleem

Arino, Joaquin

Arinstein, Arkadi

Arioli, Stefania

Arisaka, Yoshinori

Arita, Masanori

Ariyasu, Daisuke

Ariyoshi, Wataru

Ariza, Adolfo J.

Arizza, Vincenzo

Arkhipov, Vladimir I.

Armato, Ubaldo

Armellini, Elia

Armeni, Tatiana

Armiento, Angela Rita

Armstead, William M.
Armstrong, Heather

Arnao, Marino Bañón

Arnesano, Fabio

Arnhold, Juergen

Arnhold, Jürgen

Arnoult, Damien

Arock, Michel

Aroni, Sonia

Arora, Gunjan

Arosio, Paolo

Arranz, Amaia

Arrebola, Francisco A.

Arrigoni, Chiara

Arroo, Randolph

Arruda-Carvalho, Maithe

Arshad, Najla

Artalejo, Antonio R

Arteel, Gavin E.

Artero, Ruben

Artés-Hernández, Francisco

Artín-Aragón, Sagrario M

Artuch, Rafa

Artur, Mariana Aline Silva

Artyszak, Arkadiusz

Aruanno, A.

Arumäe, Urmas

Aryal, Rishi

Aryal, Uma K

Aryan, Laila

Asada, Ken

Asada, Masahito

Asadipooya, Kamyar

Asano, Atsushi

Asano, Naoki

Asaro, Fioretta

Asatryan, Liana

Ascoli Marchetti, Andrea

Aseervatham, Jaya

Asfor, Amin

Asgharian, Hosseinali

Ashida, Hitoshi

Ashihara, Eishi

Ashley, Jon

Ashley, Zoe

Ashour, Mohamed Lotfy

Ashour, Mohamed-Bassem

Ashpole, Nicole $\mathrm{M}$.

Ashraf, Ghulam Md

Ashton, Melanie

Asimaki, Angeliki

Asimakopoulos, Anastasia 
Asimakopoulos, Byron

Askes, Sven

Askjaer, Peter

Aslam, Muhammad

Aspenström, Pontus

Asquith, Christopher R. M.

Assaf, Jean-Claude

Assenza, Giovanni

Assirelli, Elisa

Astolfi, Andrea

Astolfi, Maria

Asturiano, Juan F.

Aszodi, Attila

Atabai, Kamran

Atawia, Reem T.

Athinarayanan, Shaminie

Athyros, Vasilios

Atkin, Stephen L.

Atlante, Anna

Atlasz, Tamas

Atobe, Masakazu

Atochin, Dmitriy

Atrei, Andrea

Atsushi, Matsumura

Attanasio, Chiara

Atteritano, Marco

Attia, Mohamed F.

Attila, Fehér

Attili, Anna Rita

Attri, Pankaj

Atwal, Paldeep S

Aubert, Emmanuel

Aubert, Gregory

Auburger, Georg

Auclair, Karine

Audet-Walsh, Étienne

Audi, Said

Auguet, Teresa

Augustin, Hellmut

Aunapuu, Marina

Aung, Meiji Soe

Aureli, Massimo

Aurrand-Lions, Michel

Aushev, Vasily

Aussenac, Thierry

Austin, Rachel

Autelli, Riccardo

Autry, Joseph

Avalle, Lidia

Avdeeva, Varvara

Avelange-Macherel, Marie-Hélène
Avidor-Reiss, Tomer

Avignone, Elena

Ávila, Julio

Avila, Matias

Ávila-Flores, Antonia

Aviles, Francesc Xavier

Avin, Vijay

Avino, Pasquale

Avramova, Viktoriya

Awasthi, Mayanka

Awasthi, Praveen

Awatade, Nikhil T

Awate, Sanket

Awayda, Mouhamed

Ayabe, Tatsuhiro

Ayayee, Paul Akwettey

Ayaz, Ahmet

Aydar, Ebru

Aylett, Christopher H.S.

Ayuso, José María

Azad, Obyedul Kalam

Azad, Taha

Azam, Aa Haeruman

Azarin, Kirill Vitalievich

Azarov, Jan E.

Azer, Samy A.

Azevedo, Andreia

Azevedo, Herlander

Azevedo, Jorge

Azevedo, Maria Manuel

Azimzadeh, Omid

Azuma, Chieko

Azuma, Kazuo

Azushima, Kengo

Baaße, Annemarie

Baba, Hiroko

Baba, Tsukasa

Babes, Alexandru

Babichev, Sergii

Babickova, Janka

Babii, Oleg

Babina, Magda

Babu, Anish

Babu, Dinesh

Baburamani, Ana A.

Baccarani, Umberto

Bacchus-Montabonel, Marie-Christine

Bacci, Maria Laura

Bacci, Stefano

Bacete, Laura

Bacher, Ulrike 
Bachrach, Gilad

Bachy, Christine M

Baci, Denisa

Backer, Ronald A.

Bacolla, Albino

Bacso, Zsolt

Baczkó, István

Badadani, Mallikarjun

Badaeva, Ekaterina

Badawi, Michael

Badawy, Abdulla

Badea, Nicoleta

Badea, Tudor Constantin

Bade-Doeding, Christina

Badhe, Ravindra

Badía, Roger

Badino, Paola

Bado, Igor

Badowski, Michael

Badran, Ahmed

Badrising, Umesh

Bae, Hae-Rahn

Bae, In Hwa

Bae, Sangsu

Baek, Jeong-Hwa

Baek, Jong-Suep

Baer, Patrick C.

Baeza, Alejandro

Bagal-Kestwal, Dipali R.

Bagchi, Debasis

Baghbaderani, Behnam Ahmadian

Baglivo, Ilaria

Baglole, Carolyn

Bagniewska-Zadworna, Agnieszka

Bagnoli, Paola

Bagyinszky, Eva

Bahal, Raman

Bahls, Martin

Bahn, Andrew

Bai, Renren

Bai, Shi

Baietti, Maria Francesca

Baig, Mohammad Hassan

Bailey, Adam L.

Bailey, Charles G

Bailey, Ernest

Bailly, Christian

Baino, Francesco

Bairaktari, Eleni

Baizabal, José Manuel

Baj, Andreina
Baj, Jacek

Bajaj, Jeevisha

Bajaj, Ruchika

Bajan, Sarah

Bajda, Tomasz

Bajek, Anna

Bajgain, Prabin

Bajguz, Andrzej

Bajinskis, Ainars

Bajpai, Vivek

Bajusz, Dávid

Bajusz, Izabella

Bajwa, Amandeep

Bak, Andrzej

Bakaeva, Zanda

Bakarčić, Danko

Baker, Bradley

Baker, Brent A.

Bakht, Martin

Bakhti, Mostafa

Bakillah, Ahmed

Bakker, Hans

Bakloushinskaya, Irina

Bakos, Jan

Balabanova, Larissa

Balacescu, Ovidiu

Balaguer, Patrick

Balaj, Leonora

Balakirev, Evgeniy S.

Balalaeva, Irina V.

Balan, Prabhu

Balan, SreeKumar

Balandin, Alexander A.

Balao, Francisco

Balaphas, Alexandre

Balaraman, Velmurugan

Balas, Mihaela

Balashova, Nataliya

Balázs, Varga

Balbach, Melanie

Balcar, Vladimir

Balcerczyk, Aneta

Baldanzi, Gianluca

Baldassarre, Maria Elisabetta

Baldi, Elisabetta

Baldini, Enke

Baldwin, Albert S.

Balestra, Dario

Balestrini, Raffaella Maria

Balestrini, Simona

Balkan, Wayne W. 
Balla, Jozsef

Balland, Eglantine

Ballaz, Santiago J.

Ballegeer, Marlies

Ballester, Ana-rosa

Ballini, Andrea

Ballouz, Sara

Balmer, Lois

Balne, Praveen Kumar

Baloni, Priyanka

Balsano, Clara

Balsinde, Jesus

Baltriukiene, Daiva

Bamforth, Simon

Ban, Bhupal

Ban, Yusuke

Banach, Artur

Banach, Mateusz

Banadyga, Logan

Banaś, Antoni

Banaszak, Szymon

Bandapalli, Obul Reddy

Bandopadhyay, Rina

Banegas Luna, Antonio Jesús

Banerjee, Abhinandan

Banerjee, Anwesha

Banerjee, Aparajita

Banerjee, Priya R.

Banerji, Versha

Banfi, Giuseppe

Bank, Ilan

Banke, Tue G.

Bankova, Vassya

Banks, William

Banning, Antje

Banno, Masahiro

Banoub, Joseph $\mathrm{H}$.

Bansal, Aditya

Bansal, Kuldeep K.

Banta, Lois

Banti, Christina N.

Bao, Jun-Xiang

Bao, Yongping

Baptista, Pedro Viana

Bär, Christian

Bär, Séverine

Barabasz, Wieslaw

Barabutis, Nektarios

Barajas-Martínez, H.,

Barak, Boaz

Barakat, Tahsin Stefan
Barakate, Abdellah

Baralle, Marco

Baran, Jaroslaw

Baran, Wojciech

Barancik, Miroslav

Baranova, Ekaterina

Baranowska-Bosiacka, Irena

Barash, Danny

Barashkov, Nikolay A.

Barazzuol, Lara

Barbagallo, Davide

Barbalho, Sandra Maria

Barbanti-Bròdano, Giovanni

Barbara, Breznik

Barbas, João Pedro

Barbati, Cristiana

Barbault, Florent

Barbe, Mary F.

Barberis, Elettra

Barberis, Massimo

Barbero, Francesca

Barbier, Julien

Barbieri, Antonio

Barbieri, Michelangela

Barbieri, Silvia S.

Barbonetti, Arcangelo

Barbosa, Flávia Leão

Barca, Amilcare

Barcaccia, Gianni

Barcellini, Wilma

Barcia, Carlos

Bardají, Eduard

Barding, Gregory A.

Bardova, Kristina

Bardyn, Manon

Bareja, Akshay

Bari, Elia

Barichello, Tatiana

Barik, Sailen

Barillari, Giovanni

Baritugo, Kei Anne

Barkan, Daniel

Barker, Tyler

Barki-Harrington, Liza

Barkleit, Astrid

Barlier, Anne

Barlocco, Daniela

Barlow, Jacqueline

Barlow, Linda A.

Barna, Balazs

Barna, János 
Barnabei, Agnese

Barnard, Mollie

Barnes, Jarrod

Baron, Byron

Baroncini, Damiano

Barone, Ines

Bar-Or, David

Barosi, Giovanni

Barragán, Isabel

Barral, Duarte

Barras, Frédéric

Barreca, Davide

Barreiro Iglesias, Antón

Barrero, María José

Barro, Francisco

Barroso, Madalena

Barro-Soria, Rene

Barrott, Jared

Barta, Csengele

Bartáková, Vendula

Barteková, Monika

Bartel, Sabine

Bartels, Dorothea

Bartelt, Alexander

Barth, Brian

Bartholomeeusen, Dr. Koen

Bartnik, Ewa

Bartok, Eva

Bartoli, Manuela

Bartollino, Silvia

Bartolome, Fernando

Barton, Samantha

Bartosik, Aneta Agnieszka

Bartosz, Grzegorz

Bartoszek, Agnieszka

Bartoszewski, Rafal

Bartova, Eva

Bartuzi, Damian

Barutcu, Rasim

Barvik, Ivan

Barwiolek, Magdalena

Barygin, Oleg I.

Barzilay, Joshua

Bar-Ziv, Raz

Bar-Zvi, Dudy

Baś, Bogusław

Basak, Indranil

Basalay, Maryna

Basar, Murat

Basetti, Madhu

Bashkin, James K.
Bashtrykov, Pavel

Bashur, Christopher

Basile, Teodora

Basit, Abdul

Baslam, Marouane

Basnet, Ram Manohar

Bass, Andrew

Baßler, Jochen

Bassolé, Imaël Henri Nestor

Bastmeyer, Martin

Basu, Reetobrata

Basu, Rupsa

Basu, Supratim

Baswan, Sudhir

Batchu, Ramesh

Bateman, Joesph Matthew

Bates, John T.

Bathula, Chandra Sekhar

Batish, Mona

Batool, Maria

Batorova, Angelika

Batta, Kiran

Battaglia, Eric

Battaglia, Rosalia

Battista, Sabrina

Battistelli, Cecilia

Battistelli, Michela

Battistini, Chiara

Battula, Narendra

Baucum, Anthony J.

Baud, Stephanie

Baudier, Jacques

Baudoin-Dehoux, Cécile

Bauer, Jessica

Baugh, J. A.

Baulin, Vladimir A

Baulina, Natalia

Baumann, Marcus

Baumann, Ulrich

Baumgarner, Bradley L.

Baumgart, Simon J.

Baumgarten, Martin

Baumgarten, Thomas

Baumgartner, Ulrich

Baumstark-Khan, Christa

Bauser-Heaton, Holly

Bautista, Rocío

Bawadekar, Mandar

Bawage, Swapnil

Bax, Bridget $\mathrm{E}$.

Baxter, Victoria K. 
Bay, Denice C.

Bay, Peter

Bayer, Thomas

Bayer-Skoff, Sarah M.

Baykov, Alexander

Bayona-Bafaluy, María Pilar

Bazán, Eulalia

Bazan-Socha, Stanislawa

Bazhanova, Elena D

Bazhenov, Viacheslav

Bazihizina, Nadia

Bazil, Jason

Bazrafshan, Zahra

Bazwinsky-Wutschke, Ivonne

Bazylińska, Urszula

Bear, Christine

Beard, Matthew

Beasley, Shannon

Beaumelle, Bruno

Beauvais, Wendy A.

Beaven, Anne W.

Beavis, Paul

Bebelman, Maarten P.

Beberok, Artur

Becciolini, Andrea

Becerra, Manuel

Beck, Benjamin

Beck, Hans Christian

Beck, Michael W.

Beckel, J. M.

Becker, Daniel P.

Becker, Susanne

Becker, Thomas

Becker, Yvonne

Becker-Pauly, Christoph

Becq, Fredric

Becucci, Lucia

Bedi, Deepa

Bedia, Carmen

Bedini, Emiliano

Bedini, Gianni

Bednarek, Radoslaw

Bednarski, Patrick J.

Bednarz-Knoll, Natalia

Bedon, Frank

Bedoyan, Jirair K

Beenakker, Jan-Willem

Beesley, Jonathan

Beetz, Christian

Begcy, Kevin

Begemann, Matthias
Beger, Richard

Beghini, Alessandro

Beguelin, Wendy

Behera, Smrutisanjita

Behling, Felix

Behr, Marc

Behrens, Jürgen

Behrens, Sven-Erik

Behringer, Erik

Behzadi, Payam

Beilby, Mary

Beirowski, Bogdan

Beissert, Tim

Bejarano, Ignacio

Bekeschus, Sander

Bekiari, Vlasoula

Bekri, Soumeya

Belayew, Alexandra

Belda-Palazón, Borja

Belgiovine, Cristina

Belin De Chantemèle, Eric J.

Belizario, José Ernesto

Belizna, Cristina

Bell, John

bell, Stephen

Bellan, Mattia

Bellanti, Francesco

Bellardita, Carmelo

Bellavia, Daniele

Bellei, Barbara

Bellelli, Roberto

Bellemin-Laponnaz, Stéphane

Bellien, Jeremy

Bellier, Jean Pierre

Bellini, Maria Irene

Bello, Nicholas T.

Bello, Xabier

Bello-Espinosa, Luis E.

Bellomo, Francesco

Bellucci, Stefano

Belluti, Silvia

Beloborodova, Natalia V.

Belosludtsev, Konstantin

Beloukas, Apostolos

Beltowski, Jerzy

Bemporad, Francesco

Benabdellah, Karim

Benada, Jan

Benamar, Khalid

Benarafa, Charaf

Benarba, Bachir 
Benavente, Claudia A

Benavides-Mendoza, Adalberto

Benbrook, Doris Mangiaracina

Bencharit, Sompop

Bencivenga, Stefano

Bencsik, Peter

Bendahhou, Saïd

Ben-David, Yaacov

Bende, Attila

Bender, Kevin

Bendokas, Vidmantas

Benedetti, Elisabetta

Benedetti, Francesca

Benedetti, Michele

Benedetti, Rosaria

Benedetto, Alexandre

Benelli, Roberto

Benetti, Elisa

Benfaremo, Devis

Benfeito, Sofia Ester

Benfenati, Emilio

Benhelli, Houda

Benistant, Christine

Benítez Jiménez, José Jesús

Bennet, Andrew

Bennett, Lea D.

Bennevault-Celton, Véronique

Bennewitz, Margaret F.

Benoit, Chassaing

Benoit, Matthias

Benowitz, Kyle M

Benrick, Anna

Bentel, Jacqueline M.

Bentley, T. William

Benussi, Alberto

Benveniste, Helene

Benyhe, Sandor

Benz, Roland

Ben-Ze'ev, Avri

Benzoni, Patrizia

Berardesca, Enzo

Berardi, Anna Concetta

Berardi, Emanuele

Berardo, Clarissa

Berchtold, Martin W.

Beretta, Giovanni L.

Berezhnaya, Elena

Berezovski, Maxim V.

Berezovsky, Igor

Berg, Matthew D.

Bergamo, Alberta
Bergandi, Loredana

Berg-Beckhoff, Gabriele

Bergdahl, Andreas

Bergen, Arthur

Berger, Beatrice

Berger, Slava

Berger, Wolfgang

Bergero, Roberta

Bergholz, Teresa

Bergmann, Cornelia

Bergougnoux, Véronique

Bergquist, Robert

Beringue, Vincent

Beristain, Alexander

Berka, Karel

Berkefeld, Andreas

Berlanda, Nicola

Berlec, Aleš

Berlin, K. Darrell

Bermudez, Marcel

Bernacchia, Giovanni

Bernal, Vicente

Bernal-Lopez, M. Rosa

Bernard, Amélie

Bernard, Emilien

Bernard, Marek K.

Bernardes, Nuno

Bernardi, Simona

Bernardini, Andrea

Bernardino, Raquel L.

Bernardo, Antonietta

Bernasconi, Pia

Bernatchez, Jean A.

Bernatík, Ondřej

Bernaudin, Jean-François

Berndt, Michael C.

Bernhardt, Anne

Berni, Roberto

Bernitsas, Evanthia

Bernocchi, Graziella

Bernstein, Hans-Gert

Bersano, Anna

Bertacchini, Jessika

Bertea, Cinzia

Berthelot, Laureline

Bertho, Jean-Marc

Bertho, Nicolas

Bertin, Samuel

Bertok, Sara

Bertoldi, Mariarita

Bertoli, Alessandro 
Bertorelli, Rosalia

Besalú, Emili

Besharat, Zein Mersini

Bessa, Jose

Besse, Lenka

Besser, Martin

Bessette, Barbara

Bessmertnykh-Lemeune, Alla

Besson, Thierry

Besteiro, Sébastien

Bettache, Nadir

Bettotti, Paolo

Betzel, Christian

Bevilacqua, Arturo

Beyeh, Ngong Kodiah

Beyer, Eric $\mathrm{C}$.

Beyersdorf, Friedhelm

Beznosková, Petra

BEZU, Lucillia

Bharadwaj, Vivek

Bharaj, Preeti

Bharath, Leena P.

Bhardwaj, Gourav

Bhardwaj, Rajesh

Bhat, Jaydeep

Bhat, Kamakoti

Bhat, Neha

Bhat, Owais

Bhati, Kaushal Kumar

Bhatia, Divya

Bhatia, Harsharan S.

Bhattacharjee, Apurba Krishna

Bhattacharjee, Mrinal

Bhattacharjee, Sonali

Bhattacharya, Abhisek

Bhattacharya, Anindya

Bhattacharya, Kaushik

Bhattacharya, Supriyo

Bhattacharyya, Piyali

Bhattacharyya, Sanchari

Bhattacharyya, Tapan

Bhattarai, Ajaya

Bhattarai, Gehendra

Bhattarai, Kashi Raj

Bhattarai, Krishna

Bhatti, Fazal-ur-Rehman

Bhawal, Ujjal Kumar

Bhetwal, Bhupal P.

Bhuckory, Shashi

Bhullar, Raj

Bhusal, Ram
Bhusal, Siddhi Jeewan

$\mathrm{Bi}$, Andy Chengfeng

Bi, Mingjun

Bi, Xin

Biagioni, Alessio

Biagioni, Audrey Franceschi

Bialecka, Monika

Białek, Agnieszka

Bialonska, Dobroslawa

Białowąs, Jacek

Biamonti, Giuseppe

Bianchi, Antonio

Bianchi, Fabrizio

Bianchi, Francesca

Bianchi, Vittorio

Bianco, Andrea

Bianco, Federico

Bianco, Piero

Bianco, Simona

Biancolella, Michela

Biancolillo, Alessandra

Biasucci, Giacomo

Bichet, Daniel G.

Bidault, Guillaume

Bieber, Michael

Biedermann, Thomas

Biehl, Christoph

Bielas, Rafał

Bielas, Wiesław

Bielczyk-Maczyńska, Ewa

Bielfeldt, Stephan

Bienkiewicz, Ewa Anna

Bienvenut, Willy

Bierle, Craig

Biernasiuk, Anna

Biersack, Bernhard

Bijlsma, Maarten F.

Bikbova, Guzel

Bilancio, Antonio

Bilbao, Daniel

Bilewicz, Aleksander

Bilger, Andrea

Bilic-Ćurčić, Ines

Bilińska, Zofia T.

Billard, Jean Marie

Billiald, Philippe

Billingsley, Hayley E.

Billmyre, Blake

Bilska-Kos, Anna

Bilska-Wilkosz, Anna

Bin, Bum-Ho 
Binhi, Vladimir N.

Bink, Diewertje I

Binley, James

Biondi, Antonio

Biondi, Stefania

Biondo, Luana Amorim

Biosca, Elena G.

Biressi, Stefano

Birgegård, Gunnar

Birket, Susan

Biroccio, Annamaria

Biron, David Georges

Bironaité, Daiva

Biscetti, Federico

Bischof, Joachim

Bischof, Sandra

Biscotti, Maria Assunta

Bishehsari, Faraz

Bishnupuri, Kumar S.

Bissonnette, Élyse Y.

Bissonnette, Nathalie

Biswas, Arijit

Biswas, Roopa

Bis-Wencel, Hanna

Bitonti, Maria

Bitra, Aruna

Bitto, Alessandro

Biundo, Fabrizio

BIVONA, Giulia

Bjelland, Svein

Bjelobaba, Ivana

Bjork, Bryan

Bjorklund, Robert

Blaby-Haas, Crysten E.

Black, James R.M.

Blackburn, Gavin

Blagojevic Zagorac, Gordana

Blaheta, Roman

Blalock, William

Blamires, Sean

Blanc, Etienne

Blanch, Richard J.

Blanco, Eduardo

Blanton, Cynthia

Blanvillain, Robert

Blasco Benito, Sandra

Blasi, Francesca

Blau, Jenny

Blazevic, Ivica

Blázquez, Manuel

Bleibtreu, Alexandre
Bleilevens, Christian

Blenda, Anna

Blesson, Chellakkan Selvanesan

Blitek, Agnieszka

Bloch, Sylwia

Bloch, Wilhelm

Block, Stephan

Bloise, Nora

Blomhoff, Heidi K.

Blomme, Eric

Blomme, Jonas

Blonska, Marzenna

Bloom, Kerry

Bloor, Ian

Blossey, Ralf

Blum, Jason L.

Blume, Cornelia

Blumenschein, Tharin

Blumer-Schuette, Sara E.

Blunk, Torsten

Blunt, Warren

Błyszczuk, Przemysław

Board, Mary

Boarescu, Paul-Mihai

Boase, Nathan

Boateng, Joshua

Boaventura, Paula

Bobasov, Evgenii

Bobay, Louis-Marie

Bobe, Regis

Bobich, Edward

Bobiński, Marcin

Bobis-Wozowicz, Sylwia

Bobrovskaya, Larisa

Boccaccio, Carla

Boccalatte, Francesco

Boccardo, Francesco

Boccella, Serena

Bocchinfuso, Gianfranco

Bock, Florian

Bock, Nathalie

Bocos, Carlos

Boczkowska, Maja

Bodelón-González, Gustavo

Bodine, Sue

Bodo, Enrico

Boeckel, Jes-Niels

Boege, Fritz

Boehm, Stefan

Boehme, Karen A.

Boehr, David D. 
Boekema, Bouke

Boerma, Marjan

Boeuf, Hélène

Boffano, Paolo

Bogacka, Iwona

Bogado Pascottini, Osvaldo

Bogdan, Maria

Bogdanov, Alexey M.

Boger, Heather

Bogers, Willy M J M

Boggaram, Vijay

Bøgwald, Jarl

Bohannon, Richard W.

Bohl, Delphine

Böhrnsen, Florian

Boia, Raquel

Boichuk, Sergei

Boisivon, Helene Robert

Boissan, Mathieu

Boissinot, Marjorie

Bojarczuk, Kamil

Bojarska, Joanna

Bojarski, Piotr

Bojková, Bianka

Bol, Kalijn

Boland, C. Richard

Boland, Sebastian

Bolanowski, Marek

Bölcskei, Kata

Boldison, Joanne

Boldizsár, Imre

Boldogh, Istvan

Boldrini, Luca

Bollag, Wendy

Bollong, Michael

Bollyky, Paul

Bolognin, Silvia

Bombard, Sophie

Bombardi, Cristiano

Bömer, Nils

Bommagani, Shobanbabu

Bommareddy, Praveen

Bommireddy, Ramireddy

Bonacchi, Massimo

Bonacina, Fabrizia

Bonam, Srinivasa Reddy

Bonanno, Silvia

Bonar, Emilia

Bonato, Matteo

Boncela, Joanna

Bond, Heather M.
Bond, Simon T.

Bondar, Natalia P

Bondarev, Stanislav A.

Bonduelle, Colin

Bondy, Stephen C.

Bonen, Linda

Bonetta, Dario

Bonfiglio, Rita

Bongers, Kale

Bonggi, Lee

Bongiorno, Dafne

Bonifacio, Maria A.

Bonifacio, Maria Addolorata

Bönig, Halvard

Bonin, Serena

Bonnelye, Edith

Bonnet, Crystel

Bonnet, Pascal

Bonnet, Pierre-Antoine

Bono, Federica

Bonomi, Francesco

Bonomi, Marco

Bonomini, Francesca

Bonora, Elena

Bonora, Massimo

Bonvicini, Cristian

Boomer, Jonathan S.

Boonen, Marielle

Booth, Brian

Booth, Kevin T.

Boothby, Thomas

Boppana, Nithin B.

Boquete, Teresa

Borbély, János

Borbone, Nicola

Borcan, Florin

Borchert, Glen

Borde, Valérie

Bordes, María Dolores Llobat

Bordes, Patricia

Bordignon, Vilceu

Bordin, Luciana

Borgel, Delphine

Borger, Verena

Borges, Karin

Borges, Pedro

Borghaei, Ruth C.

Borghese, Roberto

Borghi, Monica

Borgnia, Mario J.

Borgo, Christian 
Borgonovo, Gigliola

Borin, Dmitry

Boris, Pejin

Borkowska, Edyta Marta

Borkowska, Paulina

Borlee, Brad

Borlongan, Cesario Venturina

Borm, Paul

Bornancin, Frédéric

Börner, Richard

Bornhorst, Miriam

Boros, Laszlo

Borota, Ana

Boroviak, Katharina

Borovikov, Yurii S.

Borrelli, Enrico

Borschevsky, Anastasia

Borse, Vikrant

Borsetti, Alessandra

Bortey-Sam, Nesta

Bortner, Carl D.

Bortolotto, Tissiana

Bortoluzzi, Cristiano

Borzok, Maegen

Bosca, Lisardo

Boschert, Verena

Boscolo, Elisa

Bose, Debojit

Bosnar, Maja Herak

Bosnjak, Berislav

Bosse, Jens B.

Bossi, Gianluca

Bosso, Antonella

Bostan, Hamed

Bostelmann, Richard

Bostjancic, Emanuela

Botelho, João

Botella, Luisa María

Boto, Roberto A.

Bott, Alex

Botta, Luigi

Botta, Maura

Bottani, Emanuela

Bottari, Giovanni

Botti, Gerardo

Botticelli, Andrea

Botton, Alessandro

Bottone, Maria Grazia

Botturi, Andrea

Botubol-Ares, José Manuel

Boublikova, Ludmila
Bouchal, Jan

Bouché, Marina

Boucheix, Claude

Boucher, Dave

Bouckaert, Julie

Boue-Grabot, Eric

Bougea, Anastasia

Bou-Gharios, George

Boukens, Bastiaan J.

Boukpessi, Tchilalo

Boulaiz, Houria

Bouley, Richard

Boulling, Arnaud

Bouranis, Dimitris L.

Bourbousse, Clara

Bourdon, Emmanuel

Bourgault, Steve

Bourgeois, Dominique

Bourgine, Paul Emile

Bourguet, William

Bourland, Fred

Bourneuf, Emmanuelle

Bouropoulos, Nikolaos

Bourras, Salim

Bourret, Robert B.

Bouschet, Tristan

Bousquet, Guilhem

Bousset, Luc

Boutilier, Kim

Bouyer, Daniel

Bové, Jordi

Bowden, Bruce

Bowen, Mark E.

Bowerman, Melissa

Bowers, Doria F

Bowling, Sarah

Boyanova, Lyudmila

Boyd, Robert S.

Boyer, Justin G.

Boyles, Matthew S. P.

Boysen, Reinhard

Bozdag, Murat

Bozic, Josko

Bozso, Zoltan

Bozzi, Fabio

Bozzo, Gale G.

Brabet, Philippe

Bracco, Enrico

Brachner, Andreas

Bracht, Thilo

Bracken, Adrian 
Brader, Günter

Bradford, Barry

Bradford, Kent

Bradfute, Steven

Bradley, Elizabeth W.

Bradshaw, Nicholas

Bradshaw, Patrick

Bradshaw, Tracey D.

Braendle, Christian

Brainard, Michael

Braliou, Georgia

Brambilla, Vittoria

Brancato, Virginia

Branch, Matthew James

Branchini, Alessio

Brand, Korbinian

Brand, Marcus

Brandao, Bruna

Brandenburg, Klaus

Brandenburg, Lars-Ove

Brandli, Alice

Brandt, Roland

Brandusoiu, Ionut Bogdan

Branković, Ivan

Branlant, Christiane

Brantley, William A.

Brantly, Mark

Bratengeier, Cornelia

Brault, Jeffrey

Braun, Andrew P.

Braun, Frank K.

Braun, Ralf

Braun, Rudolf K.

Braun, Werner

Bräuniger, Thomas

Bravatà, Valentina

Bravo, Jeronimo

Bravo, Lidia

Bravo, Susana B.

Brazzolotto, Xavier

Bréchard, Sabrina

Brecker, Lothar

Breckwoldt, Michael

Brehm, Martin

Brehme, Marc

Breitbart, Haim

Brejnrod, Asker

Bremer, Jeroen

Brender, Jeffrey

Brenišin, Marek

Brennan, Anthony B.
Brennan, Todd V.

Brenner, Annette K.

Brenner, Gary J.

Brenner, Rolf

Brents, Lisa K.

Bresciani, Carla

Bresciani, Elena

Bresson-Bepoldin, Laurence

Brestič, Marián

Brestoff, Jonathan R.

Brett, Thomas

Breunig, Joshua

Breuzard, Gilles

Brevini, Tiziana A. L.

Brew-Appiah, Rhoda

Brewer, Matthew G.

Brezovský, Jan

Briana, Despina D.

Briand, Loic

Bridges, Christy

Brieño-Enriquez, Miguel

Brieva, Lluís

Briganti, Matteo

Brill, Richard W.

Brindisi, Matteo

Brindley, David

Brindley, Melinda

Brini, Emiliano

Brini, Marisa

Brink, Peter

Brinkmann, Volker

Brito, Luis A.

Brito, Nélida

Britt, Rodney D.

Brittan, Mairi

Britz, Margaret L.

Briz, Oscar

Brizuela-Madrid, Leyre

Broberg, Martin

Brock, James

Brodaczewska, Klaudia K.

Brodehl, Andreas

Broderick, Patricia

Brodlie, Malcolm

Broeckling, Corey

Broer, Stefan

Brogden, Graham

Brogi, Simone

Broglie, Martina

Brogna, Claudia

Brommer, Benedikt 
Brone, Bert

Brooks, Brian

Brooks, Craig R.

Brooks, Tracy A.

Brorsson, Ann-Christin

Brosche, Mikael

Brosens, Jan

Brotto, Marco

Broude, Eugenia V.

Brouwers, Jos F.

Brown, Alan B.

Brown, Angela

Brown, Christopher Lyon

Brown, Dennis

Brown, Janet E.

Brown, Laura

Brown, Nelson

Brown, Patrick

Brown, Robert J.

Brown, Ronald B.

Brownstone, Nicholas

Broza, Yoav

Brozek-Pluska, Beata

Brözel, Volker

Brozmanová, Mariana

Brozovic, Anamaria

Brozyna, Anna

Brucoli, Matteo

Bruder Do Nascimento, Thiago

Bruemmer, Jason E.

Brugarolas, Pedro

Brügger, Britta

Brugna, Myriam

Bruinsma, Bote

Brukman, Nicolas

Brule, Sybille Van Den

Brulíková, Lucie

Brullo, Chiara

Brumos, Javier

Brun, Paola

Brundage, Cord M.

Bruneel, Arnaud

Brunello, Giulia

Brunetti, Cecilia

Brunetti, Giacomina

Brunetti, Oronzo

Brunkhorst, Robert

Brunner, Susanne

Brünnert, Daniela

Bruno, Andreina

Bruno, Annalisa
Bruno, Eleonora

Bruns, Danielle R.

Bruschini, Luca

Bruserud, Øystein

Brusslan, Judy

Brust-Mascher, Ingrid

Bruzzese, Dario

Bruzzone, Santina

Brvar, Miran

Bryantsev, Anton

Brycki, Bogumil

Bryk, Paweł

Bryniarski, Krzysztof

Bryszewska, Maria

Brzeska, Joanna

Brzeziński, Krzysztof

brzoska, malgorzata michalina

Brzozowski, Tomasz

Bubu, Omonigho

Buccarelli, Mariachiara

Buccheri, Maria-Antonietta

Bucci, Cecilia

Bucciantini, Monica

Buchanan, Daniel D.

Buchberger, Alexander

Buchet, Rene

Buchheim, Judith

Büchler, Tomáš

Buchman, Vladimir

Buckel, Wolfgang

Bucki, Robert

Buckingham, Lela

Buck-Koehntop, Bethany A.

Buckley, Shannon M.

Bucolo, Claudio

Budimirovic, Dejan

Budinsky, Robert A.

Budzak, Simon

Buechler, Christa

Bueno, Clarissa

Buentello-Volante, Beatriz

Bufalieri, Francesca

Buffart, Tineke E.

Buga, Ana-Maria

Bugarin, Alejandro

Bugla-Płoskońska, Gabriela

Bugno, Ryszard

Buha, Aleksandra

Buijs, Gonda

Bujacz, Anna

Bujacz, Grzegorz 
Bujak-Gizycka, Beata

Bujdáková, Helena

Bujdoso, Geza

Bujko, Mateusz

Büki, András

Bukowska, Bożena

Bukowska, Joanna

Bukowski, Michal

Bulatov, Emil

Bulatovic, Maja

Bulboacă, Adriana Elena

Bulc, Michał

Bulfamante, Antonio Mario

Bulgakov, Victor

Bulla Jr, Lee

Bulla, Roberta

Bulli, Peter

Bullon, Pedro

Bulte, Jeff

Bultynck, Geert

Bunaciu, Rodica P

Bundschuh, Ralph A.

Bunghez, Raluca

Bunik, Victoria

Bunkin, Nikolay F.

Bunoiu, Octavian Mădălin

Bunse, Lukas

Buntov, Evgeny

Buonanno, Manuela

Burchakov, Denis I.

Burdet, Nicolas

Burdukiewicz, Michał

Burek, Malgorzata

Burga, Laura N.

Burger, Michael C

Burgess, David R.

Burgess, John

Burghardt, Kyle

Burghardt, Robert

Burgstaller, Joerg

Burkard, Natalie

Burke, Donald H.

Burke, Richard

Burke, Susan J.

Burkel, Eberhard

Burkhead, Jason

Burlacu, Alexandrina

Burleigh, Thomas D.

Burnier, Julia

Burns, David

Burns, Katherine
Burpo, Fred

Burtey, Stéphane

Burton, Zachary F.

Bus, Vincent G.M.

Busardò, Francesco Paolo

Busby, Ryan R.

Busch, Maike

Busch, Martin

Bušek, Petr

Busetto, Gian Maria

Bushakra, Jill M

Businaro, Rita

Buskbjerg, Cecilie D. R.

Bussalleu, Eva

Busse, Mandy

Büsselberg, Dietrich

Bussu, Francesco

Bustamante, Alejandro

Busto, Rebeca

Bustos, Diego Martin

Bustos, Francisco

Bustos-Martínez, Jaime

Bustuchina Vlaicu, Mihaela

Butkauskas, Dalius

Butler, Georgina S

Butler-browne, Gillian

Butnariu, Monica

Butrón, Ana

Butu, Alina

Byadgi, Omkar

Byadgi, Omkar Vijay

Bychkov, Andrey

Bychkov, Maxim L.

Byeon, Haewon

Byler, Kendall G.

Byrd, Alicia K.

Byrd-Leotis, Lauren

Byrne, Frances L.

Byun, Jonghoe

Byun, Kyunghee

Byun, Sangwon

Bzducha-Wróbel, Anna

Caaveiro, Jose

Caba, Octavio

Caballero-Garcia, Beatriz

Cabaro, Serena

Cabiscol, Elisa

Cabot, Gabriel

Cabral, Benedito J.C.

Cabré, Juan J.

Cabrele, Chiara 
Cabrera, Adoracion

Cacabelos, Ramón

Cacciola, Nunzio Antonio

Cacciotti, Ilaria

Cacciotto, Carla

Cacho Teixeira, Miguel

Cachón-Gonzalez, María Begoña

Cadario, Francesco

Cadefau Surroca, Joan Aureli

Cadelis, Melissa M.

Caenazzo, Luciana

Caetano-Anolles, Derek

Cafasso, Donata

Cafiero, Mauricio

Caglayan, Melike

Cahill, Michael

Cahoon, A. Bruce

Cahova, Monika

Cai, Bishuang

Cai, Demin

Cai, Haiyuan

Cai, Houjian

Cai, Jason

Cai, Jieru

Cai, Jiyang

Cai, Kai

Caiati, Carlo

Caiazza, Martina

Caiazzo, Elisabetta

Cailhier, Jean François

Cailliau, Katia

Cairo, Montserrat

Cairrão, Elisa

Caissard, Jean-Claude

CAKIR, Birsen

Cakouros, Dimitrios

Cakstina, Inese

Calabrese, Gianpiero

Calabrese, Vittorio

Calabria, Elisa

Calado, Ângelo

Calado, Cecília R.C.

Calamai, Martino

Calandra, Pietro

Calarco, Anna

Calcagno, Simone

Calcott, Mark J.

Calderini, Ornella

Calderone, Vito

Caldo, Kristian Mark

Caldovic, Ljubica
Calendar, Richard

Caleo, Matteo

Calero Valdayo, Patricia Maria

Cali, James

Caligiuri, Isabella

Caligo, Maria Adelaide

Calina, Daniela

Çalışkan, Gürsel

Calistri, Arianna

Calistri, Daniele

Calixto, Cristiane P.

Callaini, Giuliano

Callaway, Todd R.

Callebaut, Isabelle

Calligari, Paolo

Calogeropoulou, Theodora

Caltabiano, Rosario

Calvani, Riccardo

Calvaruso, Marco

Calvert, Jane

Calvi, Brian

Calvo, Ana Cristina

Calvo-Lobo, César

Calzada, María J.

Camacho, M. Encarnación

Camaioni, Antonella

Camara, Johanna

Cambra Bort, Josep M.

Camejo, Germán

Cameli, Paolo

Cameron, Donald

Cameselle-Teijeiro, Josè Manuel

Cametti, Cesare

Camins, Antoni

Cammisotto, Vittoria

Campa, Claudine

Campagnolo, Luisa

Campana, Davide

Campanella, Alessandro

Campanella, Michelangelo

Campbell, Bradley

Campbell, Colin

Campbell, Corey L.

Campbell, Michael

Campbell, Moray

Campbell, Susan L.

Camper, Sally

Camphausen, Kevin A.

Campiche, Remo

Campiglia, Pietro

Campilho, Ana 
Campo, Gianluca

Campodoni, Elisabetta

Campolo, Federica

Camporesi, Enrico M.

Campos, Camila D. Madeira

Campos, Catarina

Campos, Joana F.

Campos-Parra, Alma Delia

Campos-Toimil, Manuel

Camproux, Anne-Claude

Campus, Guglielmo

Campuzano, Althea

Campuzano, Oscar

Cañadas, Olga

Canal, Clinton E.

Canals, Isaac

Canas, Jose

Cañas, Rafael A.

Cancemi, Patrizia

Cancian, Mauro

Candéias, Serge

Candela, Diaz-Canestro

Candela, Héctor

Cândido Carvalho, Kátia

Cândido, Elizabete

Canela, Núria

Canepari, Monica

Canfield, Scott

Cangiano, Biagio

Canosa, Stefano

Cantaluppi, Vincenzo

Cantamessa, Simone

Cantley, Melissa D.

Cañueto, Javier

Canut, Herve

Cao, Guodong

Cao, Hieu X.

Cao, Jian

Cao, Tongyu

Cao, Xian

$\mathrm{Cao}, \mathrm{Xu}$

Cao, Zhe

Cápal, Petr

Caparello, Chiara

Caparrotta, Stefania

Capasso, Anna

Capdevila, Daiana A

Capel, Carmen

Capel, Juan

Capela E Silva, Fernando

Capell, Teresa
Capilla-González, Vivian

Capillo, Gioele

Caplan, Allan

Caponi, Silvia

Caponnetto, Angela

Capozzi, Anna

Cappadone, Concettina

Capparè, Paolo

Cappelletti, Graziella

Cappellini, Francesca

Cappello, Francesco

Cappello, Valentina

Cappoen, Davie

Cappuccilli, Maria

Capraro, Jessica

Caprini, Elisabetta

Capuano, Alessandra

Capuano, Alessandro

Caputa, Michał

Caputo, Ivana

Caraballo, Rémi

Caraballo-Rodríguez, Andrés Mauricio

Caraci, Filippo

Carafa, Vincenzo

Caraglia, Michele

Carapelli, Antonio

Carare, Roxana Octavia

Carata, Elisabetta

Carazo, Alejandro

Carberry, Patrick

Carbone, Carmine

Carbone, Fabrizio

Carboni, Lucia

Cardinale, Jens

Cardona, Fernando

Cardone, Maria Francesca

Cardoso, Bruno António

Cardoso, Isabel Santos

Cardoso, Taina

Cardozo, Licy Yanes

Carella, Angelo M

Carella, Philip

Carestia, Mariachiara

Caretta, Antonio

Caretto, Sofia

Cariboni, Anna

Cariello, Marica

Carlin, Danielle

Carlon, Marianne

Carlos, Sao-Jose

Carlotto, Silvia 
Carlyle, Becky

Carmieli, Raanan

Carmona Mejías, Rita

Carmona, F. David

Carmona, JU

Carmosino, Monica

Carnero, Amancio

Carnevale Neto, Fausto

Carol, Pierre

Caroleo, Maria Cristina

Caroppo, Ettore

Carotenuto, Marco

Carotti, Simone

Carpenter, Guy

Carpi, Sara

Carr, Christopher

Carrasco, Elisa

Carrasco-López, César

Carrella, Sabrina

Carreras, Joaquim

Carrier, Paul

Carrieri, Antonio

Carriero, Maria Vincenza

Carrillo, Jeniffer

Carrión, Mar

Carro, Eva

Carroll, Chad

Carroll, James A.

Carroll, Lara S.

Carroll, Thomas

Carrouel, Florence

Carruthers, Nicholas J.

Cartee, Gregory D.

Carter, Wayne

Carulli, Lucia

Carullo, Gabriele

Caruntu, Constantin

Caruso, Calogero

Caruso, Carla

Caruso, Cristiano

Caruso, Gerardo

Caruso, Giuseppe

Caruso, Salvatore

Carvajal, Karla

Carvajal, Micaela

Carvalho De Oliveira, Jaqueline

Carvalho, Danilo Oliveira

Carvalho, Edmund

Carvalho, Lina A.S.

Carvalho, Luísa

Carvalho, Marta S.
Carver, John A.

Casadei, Nicolas

Casadei, Raffaella

Casadio, Rita

Casado-Díaz, Antonio

Casais, Rosa

Casalino, Giuseppe

Casalou, Cristina

Casals, Gregori

Casamassimi, Amelia

Casanova, Llanos

Casanova, Nancy

Casanova-Sáez, Rubén

Casao Gascón, Adriana

Casari, Alice

Casarini, Livio

Casas, Ana M.

Casas, François

Cascella, Raffaella

Cascini, Giuseppe Lucio

Cascioferro, Stella

Caseiro, Ana Rita

Caseiro, Armando

Casellini, Carolina

Caser, Matteo

Caseys, Celine

Casini, Giovanni

Casiraghi, Antonella

Caspari, Thomas

Cassano, Domenico

Cassiman, David

Cassisi, Jeffrey E.

Cassone, Giuseppe

Castagna, Michela

Castaing, Bertrand

Castaldi, Alessandra

Castaldo, Pasqualina

Castaldo, Rossana

Castanheira, Elisabete M.S.

Castan-Laurell, Isabelle

Castaño, Justo P.

Castel, Hélène

Castellano, Mar

Castellano, María Dominguez

Castellano, Orlando

Castellanos, Ainara

Castiglioni, Bianca

Castillejo, Maria Angeles

Castillo, Francisco

Castillo, Silvia

Castillo-Gonzalez, Claudia Marcela 
Castle, Alan

Castoldi, Angela

Castren, Eero

Castriconi, Roberta

Castro, Ana

Castro, Andreia

Castro, Fidel Ovidio

Castro, Pedro Humberto

Castro, Tarsila G.

Castro-Giner, Francesc

Castro-Marrero, Jesus

Castro-Torres, Rubén Darío

Castroverde, Christian Danve M.

Casu, Gavino

Casuso-Pérez, Rafael

Catalani, Elisabetta

Cataldi, Amelia

Catalin, Bogdan

Catanzaro, Giuseppina

Catarzi, Daniela

Cate, Hugo Ten

Catez, Frédéric

Cato, Andrew C. B.

Catravas, John

Catros, Sylvain

Cattaneo, Fabio

Catto, Marco

Cauffiez, Christelle

Caufield, J. Harry

Caulfield, Thomas R.

Cauli, Omar

Causse, Mathilde

Cavalcanti, João Henrique F.

Cavalieri, Francesca

Cavalieri, Vincenzo

Cavalletto, Luisa

Cavalli, Loredana

Cavalu, Simona

Cavanaugh, Jane

Cavazzoni, Andrea

Cavé, Christian

Cazzola, Roberta

Cebova, Martina

Ceccherini, Maria Teresa

Cecchi, Irene

Cecconi, Ciro

Cecconi, Sandra

Ceci, Andrea

Cecile, Vignal

Cegolon, Luca

Cejkova, Jitka
Cekanaviciute, Egle

Celakovska, Jarmila

Celetti, Angela

Celiński, Konrad

Celsi, Fulvio

Cembrowska-Lech, Danuta

Cenac, Nicolas

Čènas, Narimantas

Cenci, Giovanni

Cenciarelli, Carlo

Cencioni, Chiara

Cendron, Laura

Cengiz, Ibrahim Fatih

Center, Rob J.

Cerchiara, Teresa

Cerchione, Claudio

Cerdán, María-Esperanza

Cereda, Cristina

Cereda, Matteo

Ceresa, Brian

Cereseto, Anna

Černá, Marie

Cernat, Andreea

Cerón-Carrasco, José Pedro

Cerrito, Maria Grazia

Ceruti, Stefania

Cervelli, Manuela

Cervelli, Tiziana

Cervera, Ana

Cervetto, Chiara

Cesaro, Annabelle

Cescutti, Paola

Cestmir, Altaner

Ceyzériat, Kelly

Cha, Hee-Jae

Cha, Hwa Jun

Cha, Hyuk-Jin

Chaanine, Antoine $\mathrm{H}$.

Chaban, Inna

Chaber, Radosław

Chabot, Benoit

Chacko, Jenu Varghese

Chadwick, Amy E.

Chae, Heeyoung

Chae, Sung-suk

Chae, Younbyoung

Chagraoui, Abdeslam

Chai, Han-ha

Chai, Hongyan

Chai, Renjie

Chai, Toby C. 
Chaitanya, Saurabh

Chaki, Mounira

Chaklader, Reaz

Chakrabarti, Subhadeep

Chakraborty, Arijit

Chakraborty, Indranil

Chakraborty, Raja

Chakraborty, Sayan

Chakravorty, David

Chalard, Pierre

Chałas, Renata

Chałasiński, Grzegorz

Challa, Dilip

Challagundla, Kishore

Challet, Etienne

Chalovich, Joseph M.

Chami, Belal

Chami, Mounia

Chamuleau, Robert A.F.M.

Chamulitrat, Walee

Chan, Ben C. L.

Chan, Edward D.

Chan, K.L.

Chan, Koon-Ho

Chan, William K.

Chan, Yau Kei

Chan, Yin-Ching

Chand, Kirat K.

Chand, Vaibhav

Chandrasekar, Indra

Chandrasekaran, Vidya

Chandrasekharan, Bindu

Chang, Audrey N.

Chang, Cheng-Chang

Chang, Chia-che

Chang, Chia-Jung

Chang, Chien-Chung

Chang, Ching-Jin

Chang, Chuang-Rung

Chang, Hai-Chou

Chang, Hao-Hueng

Chang, Hao-Xun

Chang, Hsi

Chang, Hui-Yun

Chang, Hyun

Chang, Ing-Feng

Chang, Jer-Ming

Chang, Jia-Feng

Chang, Jui-Cheng

Chang, Ke-Vin

Chang, Kuo-Hsuan
Chang, Li-Ching

Chang, Ling-Chu

Chang, Long-Sen

Chang, Nai-Jen

Chang, Shu-Chun

Chang, Shun-Fu

Chang, Te-Sheng

Chang, Tzu-Ching

Chang, Wei Chiao

Chang, Wei-Min

Chang, Won Hyuk

Chang, Yu-Chan

Chang, Yu-Chao

Chang, Yun Sil

Chang, Yu-Wei

Chao, Cecilia

Chao, Chia-Ter

Chao, Chien-Ming

Chao, K.S. Clifford

Chao, Yin Xia

Chapoval, Svetlana P.

Chappell, Grace

Chappell, John

Chappell, Keith

Chappell, Mark C.

Chapple, Sarah

Chapuis, Julien

Charles Jacob, Harrys Kishore

Charles Richard, John Lalith

Charles, Philip

Charlton, Amanda

Charlton, Clivel

Charriaut-Marlangue, Christiane

Chase, Christine D.

Chastre, Éric

Chatain, Nicolas

Chatrikhi, Rakesh

Chatterjea, Devanani

Chatterjee, Anushila

Chatterjee, Ishita

Chatterjee, Madhumita

Chatterjee, Nimrat

Chatterjee, Sampurna

Chatterjee, Som S.

Chatterjee, Subroto

Chaturvedi, Palak

Chatzaki, Ekaterini

Chaudhari, Kiran

Chaudhri, Virendra

Chauhan, Bharesh

Chauhan, Harsh 
Chauhan, Neeraj

Chaurasiya, Shyambabu

Chauveau, Lise

Chavan, Hemantkumar

Chavarro Fierro, Martha Carolina

Chaveroux, Cedric

Chaves, Elena

Chaves, Susana Rodrigues

Chaves-Martinez, Felipe Javier

Chavez, Francisco

Chawengsaksophak, Kallayanee

Checa, Antonio

Checchetto, Vanessa

Chekanov, Konstantin

Chelli, Riccardo

Chemello, Liliana

Chemtob, Sylvain

Chen, Alan A.

Chen, Chao-Jung

Chen, Charles

Chen, Chen

Chen, Chien-Chin

Chen, Chi-Long

Chen, Chin-Chuan

Chen, Chiung-Mei

Chen, Chun-Chi

Chen, Chung-Ming

Chen, Chun-Han

Chen, Danica

Chen, Der-Yuan

Chen, Fure-Chyi

Chen, Gang

Chen, Guijie

Chen, Hansen

Chen, Hao

Chen, Haotong

Chen, Haoyuan

Chen, $\mathrm{Hu}$

Chen, Hui

Chen, Hui-Chen

Chen, Jhy-Der

Chen, Ji

Chen, Jieli

Chen, Jinbiao

Chen, Jin-Shuen

Chen, Junping

Chen, Jyh-Ping

Chen, Kaihong

Chen, Kuan-Fu

Chen, Kuan-Wei

Chen, Kuo-Hu
Chen, Lianmin

Chen, Li-Han

Chen, Lin

Chen, Linyi

Chen, Lisong

Chen, Lixian

Chen, Mei-Jou

Chen, Miao-Hsueh

Chen, Ming

Chen, Mu-Kuan

Chen, Pei-Wen

Chen, Qi

Chen, Qian

Chen, Qun

Chen, Sean Chun Chang

Chen, Sheng-Hung

Chen, Shih-Chieh

Chen, Shih-Chu

Chen, Shih-Kuo

Chen, Shin-Cheh

Chen, Shukun

Chen, Shuo-Bin

Chen, Shuyang

Chen, Si-Chong

Chen, Suet Nee

Chen, Suzie

Chen, Szu-Tah

Chen, Tai-Heng

Chen, Tzong-Yueh

Chen, Vincent

Chen, Wei

Chen, Wei-Jung

Chen, Weiqin

Chen, Wei-Sheng

Chen, Wei-Yu

Chen, Wenchun

Chen, Wen-Ying

Chen, Xi

Chen, Xiaoyan

Chen, Xingcheng

Chen, Xingqi

Chen, Xiongwen

Chen, $\mathrm{Xu}$

Chen, Yao-tseng

Chen, Yi-Chun

Chen, Yih-Fung

Chen, Yih-Yuan

Chen, Yiliang

Chen, Yi-Wen

Chen, $\mathrm{Yu}$

Chen, Yuan-Chuan 
Chen, Yuan-Tsung

Chen, Yu-Chih

Chen, Yung-Chih

Chen, Yun-Ju

Chen, Zhonghua

Chen, Zhongzhi

Chénais, Benoit

Cheng, Chien-Yu

Cheng, Chunming

Cheng, Fong-Yu

Cheng, Haili

Cheng, Haizi

Cheng, Henrique

Cheng, Heung-Chin

Cheng, Hong Sheng

Cheng, Hung-Chi

Cheng, Jya-Wei

Cheng, Ming-Huei

Cheng, Peilin

Cheng, Shih-Ping

Cheng, Wei-chieh

Cheng, Xiang

Cheng, Xiaodong

Cheng, Xinlai

Cheng, Yi-Sheng

Cheng, Yu-Jung

Cheon, Yong-Pil

Cheong, Heesun

Chereddy, Kiran

Cherkaoui Malki, Mustapha

Chern, Chi-Liang

Chern, Ming-Kai

Chernikov, Oleg

Chernoff, Yury

Chernova, Tatiana

Cherry, Jonathan

Cheruzel, Lionel

Chesney, Edward

Chester, Adrian $\mathrm{H}$

Cheung, Ming-Yan

Cheungpasitporn, Wisit

Chevalier, Christophe

Chevalier, François

Chevillard, Christophe

Chevret, Edith

Chhabra, Gagan

Chhabra, Yash

Chi, Celestine N.

Chi, Gerald

Chi, Yu-Jen

Chiang, Chi-Ling
Chiang, Hsiu-Mei

Chiang, Hung-Lung

Chiang, Meng-Tsan

Chiang, Ming-Ko

Chiang, Naijung

Chiang, Tung-chin

Chiang, Weichung

Chiang, Yi-Ting

Chiang, Yu-Chung

Chiang-Ni, Chuan

Chiapella, Jorge

Chiara, María-Dolores

Chiaraluce, Roberta

Chiarella, Emanuela

Chiavaroli, Annalisa

Chibalin, Alexander V.

Chieffi, Paolo

Chien, Chianshiu

Chien, Jeremy

Chiesa, Giulia

Chiesa, Mattia

Chifiriuc, Mariana Carmen

Chifiruc, Mariana Carmen

Chigbu, De Gaulle I

Chighizola, Cecilia B

Chim, Shek Man

Chimenti, Isotta

Chimienti, Guglielmina Alessandra

Chin, Kok Yong

Chin, Young-Won

China, Arnab

Chinchilla Salcedo, Nuria

Chino, Marco

Chintala, Sreenivasulu

Chintalapudi, Sumana

Chioccarelli, Teresa

Chiocchetti, Annalisa

Chiou, Chun-Tang

Chiou, Shih-Hwa

Chiriacò, Maria Serena

Chiricozzi, Elena

Chisari, Emanuele

Chitale, Shalaka

Chitarra, Walter

Chitraju, Chandramohan

Chittori, Sagar

Chiu, Ching-Feng

Chiu, Hua-Sheng

Chiu, Joanna

Chiu, Ka Fung Peter

Chiu, Ming-Jang 
Chiu, Norman H. L.

Chiu, Tsai-Hsin

Chiurazzi, Maurizio

Chivero, Ernest

Chiyoda, Tatsuyuki

Chizhov, Alexander O.

Chizzolini, Carlo

Chladek, Grzegorz

Chlichlia, Katerina

Chmielarz, Piotr

Chmielewska, Joanna

Chmielewski, Michał

Chmyrov, Andriy

Chng, Shu Sin

Cho, Hee Jun

Cho, Jae Youl

Cho, Jaehoon

Cho, Jaehyung

Cho, Je-Yoel

Cho, Jung Sook

Cho, Kinsang

Cho, Kwang-Jin

Cho, Nicole A.

Cho, Sangyun

Cho, Tae-Ju

Cho, Won Kyong

Chockalingam, Sriram P.

Choe, Eun Sang

Chohnan, Shigeru

Choi, Cheol Yong

Choi, Dong-Kug

Choi, Hojung

Choi, Hong-il

Choi, Hosoon

Choi, Hyun Jin

Choi, Inho

Choi, Jae-Hong

Choi, Jeong June

Choi, Jeong-Hwa

Choi, Pui Wah

Choi, Sangchun

Choi, Se-Young

Choi, Soo-Kyoung

Choi, Tae Gyu

Choi, Won Hyung

Choi, Young Min

Choleva, Lukas

Cholkar, Kishore

Chong, Cher-Rin

Chong, Gun Oh

Chong, Irene Yu-Shing
Choo, Hyo-jung

Chorianopoulou, Styliani N.

Chorostecki, Uciel

Choshi, Tominari

Chou, Chih-Hung

Chou, Feng-Cheng

Chou, Tsung-Han

Choudhary, Sanjeev

Choudhuri, Avik

Choudhuri, Subhadip

Choudhury, Swarup Roy

Chow, James

Chow, Simon Kwoon-Ho

Chow, Tahsin J.

Chowdhury, Farhan

Chowdhury, Indrajit

Chowdhury, Nityananda

Chowdhury, Ratul

Chowdhury, Sabiha

Choy, Francis Y.M.

Christ, Torsten

Christensen, Jørn B

Christensen, Lars P.

Christenson, Lane $\mathrm{K}$.

Christians, Elisabeth

Christie, Graham

Christodoulou, Maria-Ioanna

Christoffers, Michael

Christopoulos, Petros

Christou, Georgios A.

Christov, Nikolai K.

Chronakis, Nikos

Chroni, Angeliki

Chrousos, George P.

Chrysanthopoulou, Akrivi

Chtarbanova-Rudloff, Stanislava

$\mathrm{Chu}$, Chenggen

$\mathrm{Chu}$, Dinh Toi

$\mathrm{Chu}$, I-Ming

$\mathrm{Chu}$, Shi-Jye

$\mathrm{Chu}$, Sin Tak

Chu, Xiangping

Chuah, Seng-Kee

Chuang, Hung-Yi

Chuang, Ming-Lung

Chuang, Wan-Long

Chubanov, Vladimir

Chubarev, Vladimir N.

Chueh, Pin Ju

Chulanov, Vladimir

Chul-sung, Huh 
Chun, Kyung-Soo

Chung, Bo Young

Chung, Byung Min

Chung, Chi-Li

Chung, Ching-Hu

Chung, Geehoon

Chung, Hakjae

Chung, Hsiaohang

Chung, Hwan-Suck

Chung, Jay H.

Chung, Jayong

Chung, Ki Wha

Chung, KwiMi

Chung, Man-Kyo

Chung, Tae Nyoung

Chung-Davidson, $\mathrm{Yu}$-Wen

Chungying, Tsai

Chwalek, Karolina

Chwalibóg, André

Chwil, Mirosława

Chworos, Arkadiusz

Ciaccio, Marcello

Ciacka, Katarzyna

Ciafrè, Silvia Anna

Ciancetta, Antonella

Cianci, Antonio

Cianciolo, Giuseppe

Cianflone, Eleonora

Ciani, Maurizio

Ciarmiello, Loredana

Ciavarella, Carmen

Cicala, Carla

Cicalini, Ilaria

Ciccarelli, Michele

Cicchini, Carla

Ciccodicola, Alfredo

Ciccone, Lidia

Cichowska-Kopczyńska, Iwona

Ciciliot, Stefano

Ciclitira, Paul

Cicone, Francesco

Cid, Victor J

Cid-Arregui, Angel

Cielecka-Piontek, Judyta

Ciemerych-Litwinienko, Anna

Ciereszko, Iwona

Ciesielska, Anna

Ciesielski, Wojciech

Cifelli, Pierangelo

Cifone, Maria Grazia

Çiftçi, Halil İbrahim
Cifuentes, Daniel

Cigana, Cristina

Cignarella, Andrea

Cil, Onur

Cilurzo, Felisa

Cimmino, Giovanni

Cimpean, Anca-Maria

Cîmpean, Anişoara

Cinelli, Patrizia

Ciobica, Alin

Ciofi-Baffoni, Simone

Cione, Erika

Ciornea, Elena Todirascu

Cipak Gasparovic, Ana

Cipak, Lubos

Cipolloni, Luigi

Ciranna, Lucia

Cirillo, Giuseppe

Ciriza, Jesus

Cirmi, Santa

Ciszewski, Wojciech Michał

Citelli, Marta

Civiero, Laura

Ciz, Milan

Clanchy, Felix

Cłapa, Tomasz

Claridge, Jolyon K

Clarimon, Jordi

Clark, Barbara J

Clark, Georgina

Clark, Natalie M

Clarke, Anthony

Clarke, Christopher

Clarke, Jonathan

Clarke, Lane L.

Clarke, Penny

Claro, Carmen

Classen, Carl Friedrich

Clement, Albrecht

Clement, Cristina

Clement, Joachim

Clément, Nathalie

Clifton, Nicholas

Clifton, Peter

Climent Salarich, Montserrat

Clodt, Juliana Isabel

Close, Dan

Cluzeau, Thomas

Cobbs, Charles

Cobley, James

Coccaro, Nicoletta 
Cocco, Stefania

Cocovi-Solberg, David J.

Coelho, Antonio Victor Campos

Coelho, Ricardo

Coelhoso, Isabel

Cofelice, Martina

Cogato, Alessia

Cognasse, Fabrice

Cohen, Guy

Cohen, Justus B.

Cohen, Michael S.

Coiffard, Laurence J M

Cojocaru, Florina

Coker, Olabisi Oluwabukola

Colangelo, Donato

Colanzi, Antonino

Colasante, Gaia

Colasanti, Roberto

Colcombet, Jean

Cole, David K.

Cole, Kathryn E.

Cole, Marsha

Coleman Jr, Leon Garland

Coleman, Cynthia

Coleman, Jonathan A.

Coleman, Michael

Coleman, Mitchell C.

Coleman, Nichola J.

Coleman, Paul

Coletta, Massimo

Colitti, Monica

Coll, Monica

Colla, Emanuela

Collart-Dutilleul, Pierre-Yves

Colleluori, Georgia

Collina, Simona

Collins, Nicholas

Collongues, Nicolas

Collura, Ada

Colnaghi, Luca

Cologna, Stephanie

Colombatto, Piero

Colombeau, Ludovic

Colonna, Giovanni

Coluccia, Mauro

Colussi, Silvia

Comai, Giorgia

Combaret, Lydie

Combarnous, Yves

Combet, Christophe

Comelli, Elena
Comez, Lucia

Comincini, Sergio

Comizzoli, Pierre

Commault, Audrey S.

Comoletti, Davide

Conan, Francoise

Conceição, Natércia

Conciatori, Fabiana

Conde, Carlos

Conde, Javier

Confaloni, Anna Maria

Confavreux, Cyrille

Conigliaro, Alice

Coniglio, Salvatore J.

Connell, Gregory

Connell, Terry D.

Connor, Mark

Consolati, Giovanni

Console, Lara

Consonni, Francesca Maria

Constantinescu-Aruxandei, Diana

Constantinos, Papagiannitsis

Constantin-Teodosiu, Dumitru

Contaldo, Nicoletta

Conte, Claudia

Conte, Ivan

Conte, Mariarosaria

Conteduca, Vincenza

Conti, Giovanni

Conti, Stefania

Conti, Valeria

Contino, Marialessandra

Contreras, Osvaldo

Contreras, Rubén G.

Contursi, Annalisa

Convertini, Paolo

Conzuelo, Felipe

Cook, Leah

Cooke, Megan

Coombs, Melanie

Coon, Steven L.

Cooper, Elizabeth A

Cooper, Kerry

Copolovici, Dana Maria

Coppedè, Fabio

Coppola, Vincenzo

Coquerel, David

Coras, Roxana

Corazzari, Marco

Corbet, Cyril

Corbo, Vincenzo 
Corcoran, Jacob

Corcoran, Jennifer

Cordani, Marco

Cordani, Nicoletta

Cordano, Christian

Cordaro, Marika

Cordaro, Massimo

Cordeddu, Viviana

Cordero-Llana, Óscar

Cordes, Nils

Cormio, Antonella

Cornelius, Denise

Cornillet, Martin

Corrado, Chiara

Corrado, Giandomenico

Corrado, Giovanni

Corre, Sébastien

Correia Coelho, Ana Cláudia

Correia, Sónia C.

Correia-de-Sá, Paulo

Corridore, Denise

Corsalini, Massimo

Corsaro, Alessandro

Corsaro, Maria Michela

Corso, Giovanni

Cortay, Hélène

Cortes, Andres J.

Cortés, Kamila Caraballo

Cortes, Natalie

Corthay, Alexandre

Corti, Chiara

Corti, Olga

Cortis, Pierluigi

Corvis, Yohann

Cos, Paul

Cosenza, Maria

Coseri, Sergiu

Cosín-Roger, Jesús

Cossu, Davide

Costa, Clotilde

Costa, Julia

Costa, Marcello

Costa, Raquel

Costa, Roberto

Costa, Rui

Costa, Vera Marisa

Costa, Viviana

Costantini, Claudio

Costantini, Erica

Costantino, Claudio

Costa-Pinto, Ana Rita
Costarelli, Leopoldo

Costea, Daniela Elena

Costello, Eithne

Costoya, Jose A.

Cote, Patrice

Cotella, Diego

Cotoras, Darko

Cotter, Thomas G.

Cottini, Francesca

Couce, María D.

Couce, María Luz

Couch, Yvonne

Coudert, Amélie E

Cougnoux, Antony

Coulouarn, Cédric

Coulson-Thomas, Vivien Jane

Coumans, Joëlle V. F.

Coureux, Pierre Damien

Court, Karem A.

Courtois, Gilles

Cousins, Fiona

Coussons, Peter

Coutant, Frédéric

Coutinho, Paula

Coutinho, Paulo José Gomes

Coutinho-Silva, Robson

Couttas, Timothy A.

Couture, Réjean

Couvineau, Alain

Covarrubias, Alejandra

Covo, Shay

Cowman, Mary

Cox, Michael

Cox, Richard Aras (Aaron)

Cozza, Giorgio

Cozzani, Emanuele

Craig, Morgan

Cram, Erin J

Cravero, Maria Carla

Crawford, Bryan D.

Crawford, Emily D.

Crawford, Lisa

Creasy, Caretha

Crepaldi, Tiziana

Crescenzi, Anna

Crescenzi, Carlo

Crescenzi, Marco

Crescioli, Clara

Crespan, Emmanuele

Crespo, Francisco

Crespo, Vicente 
Crestani, Maurizio

Cretoiu, Dragos

Crevenna, Alvaro

Crevillén, Pedro

Cribbs, Adam

Crichton, Robert R.

Cridge, Andrew

Crisan, Luminita

Crisp, Peter

Crispi, Stefania

Crisponi, Guido

Crisponi, Laura

Cristescu, Rodica

Cristofani, Riccardo

Cristofaro, Raimondo De

Croce', Lory Saveria

Crocetti, Letizia

Crona, Daniel

Crosas, Bernat

Crosatti, Cristina

Croset, Martine

Cross, Michael

Crotti, Tania

Crozatier-Borde, Michele

Crucho, Carina

Crucitta, Stefania

Crupi, Mathieu J.F.

Crusats, Joaquim

Crus-Topete, Diana

Cruz, Carla

Cruz, Homero Reyes De La

Cruz, Juan C.

Cruz, Rodrigo

Cruz, Rui M.S.

Cruz-Lopez, Olga

Csabafi, Krisztina

Csámpai, Antal

Csepany, Tunde

Csernok, Elena

Csik, Gabriella

Csiszar, Agnes

Csősz, Éva

Cuajungco, Math

Cubellis, Maria

Cubero, Francisco Javier

Cubo, Leticia

Cucchiarini, Magali

Cucinotta, Francis

Cuenda, Ana

Cuesta, Candela

Cui, Haitao
Cui, Qingbin

Cui, Rongfeng

Cui, Tiantian

Cui, Yanxiang

Cui, Ye

Cui, Zhouqi

Cuif, Jean-Pierre

Cukrowska, Božena

Cullen, Paul

Cumbo, Cosimo

Cumming, Mathew Hoani

Cummings, Brian S.

Cummins, Theodore

Cunha, Rodrigo A.

Cunnane, Stephen

Cunningham, Amy

Cunningham, Madeleine W.

Cunningham, Margaret Rose

Cuppoletti, John

Curci, Pasquale Luca

Curcic, Marijana

Curcio, Alberto

Curran, Christine

Curreli, Sabrina

Currò, Monica

Curtale, Graziella

Curti, Antonio

Curtin, James F.

Curtis, Anne

Curtis, Brian R.

Curtis, Marion

Cusick, Kathleen D.

Custodio, Catarina

Cutler, Christopher W

Cutler, Mary Lou

Cutone, Antimo

Cuypers, Bart

Cvetkovska, Emilija

Cycoń, Mariusz

Cyganek, Lukas

Cymbaluk, Aneta

Cytlak, Urszula

Czaja-Bulsa, Grażyna

Czajkowski, Robert

Czakó, Márta

Czapik, Agnieszka

Czaplewski, Cezary

Czarna, Malgorzata

Czarnecka, Anna

Czechowski, Piotr Oskar

Czeiter, Endre 
Czerwinska, Monika

Czerwiński, Marcin

Czigany, Zoltan

Czogalla, Aleksander

Czuczwar, Stanisław

Czystowska-Kuzmicz, Malgorzata

Czyz, Anna

Czyż, Jarosław

Czyż, Katarzyna

Czyz, Malgorzata

Czyżewski, Krzysztof

Czyżnikowska, Żaneta

D' Acunto, Mario

D'Acquarica, Ilaria

D'Acquisto, Fulvio

D'Agostino, Carmine

D'Agostino, Massimo

D'Agostino, Paul

D'Agostino, Vito Giuseppe

D'Aguanno, Simona

D'Alessandra, Yuri

D'alessio, Alessio

D'Alò, Francesco

D'Ambrosi, Nadia

D'Amelia, Vincenzo

D'Amelio, Raffaele

D'Amico, Ramona

D'Amora, Ugo

D'anca, Marianna

D'Angelo, Armando

D'Angelo, Livia

D'Angelo, Michele

D'Anneo, Antonella

D'arcy, Pádraig

D'Ardes, Damiano

D'Argenio, Valeria

D'Autreaux, Benoit

D'Costa, Vanessa

D'Elios, Mario Milco

D'Haese, Patrick

D'Onofrio, Grazia

D'Orazi, Valerio

D'Orsi, Beatrice

D'Souza, Rochelle C. J.

D’Ursi, Anna Maria

Da Costa, Rui M. Gil

Da Lage, Jean-Luc

Da Silva E Silva, Daniel

Da Silva, Anabela Bernardes

Da Vià, Matteo

Dabbou, Sihem
Dabdoub, Alain

Dabiri, Yasamin

Dabrowski, Filip

Dabrowski, Michal J.

Dacarro, Giacomo

Dacheux, Jean-Louis

Dachineni, Rakesh

Dachs, Gabi

Daci, Armond

Dada, Laura

Dadachova, Ekaterina

Dadashi-Silab, Sajjad

Dagmara, Malina

Dagur, Raghubendra S.

Dahan, Albert

Dahiya, Rajiv

Dahl, Jan-Ulrik

Dai, Chia-Yen

Dai, Jiangkun

Dai, Wei

Daimi, Houria

Dajas-Bailador, Federico

Dal Ben, Matteo

Dal Corso, Alberto

Dal Prá, Ilaria

Dalal, Vijit

Dalbeni, Andrea

Dale, Erica A.

Dalhaimer, Paul

Dali-Youcef, Nassim

Dall'Olio, Fabio

Dalla Pozza, Elisa

Dalla, Emiliano

Dallinger, Reinhard

Dama, Murali

Damalanka, Vishnu

Damaziak, Krzysztof

Damberger, Fred

Damerval, Catherine

Damia, Giovanna

Damiani, Daniela

Damiani, Giovanni

Damiano, Fabrizio

Damiano, Sara

Damkier, Helle

Dandawate, Prasad

Dandekar, Thomas

Dane, Fenny

Daniel, Yoseph

Daniela, Ailincai

Daniele, Aurora 
Daniellou, Richard

Danielsen, Michael

Danilenko, Michael

Danilenko, VN

Daniluk, Urszula

Danisa, Olumide A.

Danisovic, Lubos

Danková, Zuzana

Dannaoui, Eric

Dansette, Patrick M.

Dantuma, Nico

Danyluk, Jean

Dar, Mohd Saleem

Dar, Wasim A.

Daras, Gerasimos

Dardis, Andrea

Dario, Brunetti

Dark, Michael

Daroszewski, Jacek

Darras, Anastasios

Darreh-Shori, Taher

Darst, Seth A.

Dartt, Darlene

Darvell, Brian

Das, Abhirup

Das, Aditi

Das, Arabinda

Das, Arup Kumar

Das, Joydip

Das, Kumuda C.

Das, Rupali

Das, Sabyasachi

Das, Subha

Das, Sudip

Das, Viswanath

Dasgupta, Kasturi

Dasgupta, Piyali

Dash, Biraja

Dash, Chandravanu

Dash, Ranjeet Prasad

Dastsooz, Hassan

Daszkowska-Golec, Agata

Datki, Zsolt

Datta, Anita N.

Datta, Arpita

Datta, Kausik

Datta, Poulami

Datta, Rupsa

Datta, Sandipan

Daubon, Thomas

Daudé, David
Daugrois, Jean-Heinrich

Dave, Sandeep

Daveluy, Steven

Daverey, Amita

David, Laure

David, Robert

Davidova, Irene

Davidson, Colin

Davidson, Cristin

Davie're, Jean-Michel

Davies, Kevin

Davies, Nigel

Davis, Erica E.

Davis, Keith R.

Davis, Mellar P.

Davis, Michael E.

Davis, Randall L.

Davis, Richard P.

Davoodi, Pooya

Davtyan, Aram

Davuluri, Gangarao

Dawood, Mahmoud A.O.

Dawra, Rajinder

Day, Andrew S.

Day, David

Day, Regina M.

Dayal, Sanjana

Daza-Navarro, Paula

De Almeida, Rodrigo Coutinho

De Almodóvar, José Mariano Ruiz

De Amicis, Francesca

De Angelis, Antonella

De Araujo, Elvin

De Aza, Piedad N.

De Baaij, Jeroen

De Barrios, Oriol

De Bellis, Luigi

De Benedetti, Pier G.

De Benedetto, Anna

De Bernardi Schneider, Adriano

De Biase, Daniela

De Bock, Katrien

De Breyne, Sylvain

De Carlis, Luciano

De Caro, Viviana

De Carvalho, Carla C. C. R.

De Chiara, Francesco

De Chiara, Letizia

De Cock, Diederik

De Falco, Elena

De Falco, Valentina 
De Felice, Francesca

De Francesco, Ernestina Marianna

De Francesco, Francesco

De Francesco, Raffaele

De Francisco, Seyla

De Geest, Bart

De Graaf, Coen

De Guia, Roldan M.

De Jesus Pereira Godinho Velada, Isabel

De Jong, Steven

De Jonge, Hugo

De Julián Ortiz, Jesus

De Koning, Leanne

De Kruif, John

De Kruijff, Robin M.

De La Fuente, Hortensia

De La Lastra, José Manuel Pérez

De La Luz Garcia-Hernandez, Maria

De La Puente, Pilar

De La Torre, Jose Garcia

De La Vega, José Manuel García

De Las Heras Montero, Javier Adolfo

De Lellis, Laura

De Lorenzo, Mariana S

De Luca, Anastasia

De Luca, Giuseppina

De Luca, Paola

De Luna-Bertos, Elvira

De Marchi, Tommaso

De Martin, Rainer

De Martinis, Massimo

De Martino, Marco

De Matteis, Rita

De Matteis, Valeria

De Meyts, Pierre

De Mieri, Maria

De Miglio, Maria Rosaria

De Moor, Cornelia

De Munain, Adolfo López

De Nisco, Nicole J.

De Nova, Pedro J.G.

De Oliveira Carvalho, Carla Roberta

De Oliveira, Cláudio

De Paepe, Boel

De Paiva, Cintia S.

De Palma, Raffaele

De Paola, Domenico

De Paolis, Angelo

De Pasquale, Valeria

De Pastena, Matteo

De Pauw, Edwin
De Pinto, Maria

De Rasmo, Domenico

De Re, Vallì

De Reijke, T.M.

De Rienzo, Assunta

De Robertis, Mariangela

De Rocquigny, Hugues

De Rooij, Dirk

De Rosa, Maria Cristina

De Rosa, Viviana

de Rosales, Rafael T.M.

De Silva, Franklyn

De Simone, Angela

De Smaele, Enrico

De Sousa-Coelho, Ana Luísa

De Spiegelaere, Ward

De Stefano, Rosa

De Summa, Simona

De Teresa, Jose-Maria

De Tullio, Mario C.

De Vicente, Juan Carlos

De Vita, Alessandro

De Vita, Daniela

De Vito, Danila

De Vos, John

De Vries, Carlie

De Vries, Teun

De Waard, Michel

De Waard, Vivian

De Zinis, Luca Oscar Redaelli

De Zotti, Marta

De, Pradip

De, Tanima

Dealy, Caroline N.

Dean, Michael

Dean, Olivia M

Deans, Rebecca

Deb, Subrata

De-Bandt, Jean-Pascal

Debatin, Klaus-Michael

Debela, Ahmed M.

Dębowski, Dawid

Dębska, Bernardeta

De-Chiara, Loretta

Decorti, Giuliana

Decuypere, Jean-Paul

Ded, Lukas

Dedhar, Shoukat

Dedkova, Elena N.

Deelman, L.E.

Deep, Gagan 
Deepak, Janaki

Deftereos, Georgios

Degani, Gad

Deganutti, Giuseppe

Degola, Francesca

DeGregorio, Danilo

Dei, Silvia

Deineko, Elena Victorovna

Deive, Francisco

Deja, Stanislaw

Dejeu, Jérôme

Dekker, Marloes

Del Angel, Rosa María

Del Boccio, Piero

Del Fattore, Andrea

Del Fresno Sánchez, Carlos

Del Gaudio, Costantino

Del Giacco, Luca

Del Giudice, Rita

Del Hoyo Martínez, Carmen

Del Prete, Annalisa

Del Río Celestino, Mercedes

De-la-Casa-Almeida, María

Delannoy, Philippe

DeLaurier, April

Delebarre, Christophe

Deleo, Francesco

Delfino, Domenico V.

Delgado Palacio, Susana

Delgado, Daniel Ricardo

Delgado, María

Delgado-Esteban, Maria

Delgado-Ruiz, Rafael

Delhomme, Nicolas

Delhommel, Florent

Delicado, Esmerilda G.

Delisle, Jean-Sébastien

Delitala, Alessandro

Deliyanti, Devy

Dell'Era, Patrizia

Dell'olmo, Eliana

Della Bartola, Michele

Della Porta, Giovanna

Della Ragione, Fulvio

Della Torre, Emanuel

Della Torre, Sara

DellaGreca, Marina

Delle Monache, Simona

Dellepiane, Sergio

Dellero, Younès

Dellis, Olivier
Dello Ioio, Raffaele

Delmaghani, Sedigheh

Delorme, Vincent

Del-Rio Celestino, Mercedes

DeLuca, Luigi

Demartini, Chiara

Dembowski, Jill

Dembska, Anna Renata

Deme, Pragneya

Demey, Hary

Demir, Fatih

DeMirci, Hasan

Demonbreun, Alexis

Dempski, Robert

Demyanenko, Svetlana A.

Demyanets, Svitlana

Demyda-Peyrás, Sebastián

Den Blaauwen, Tanneke

Denegri, Bernard

Dengler, Franziska

Denham, Mark

Deniaud, Aurélien

Denina, Marco

Dennington, Simon

Denovan-Wright, Eileen M.

Densham, Ruth

Denti, Michela A.

Deore, Prashant $S$.

Depalma, Ralph G.

Depeint, Flore

DePinho, Ronald A.

Depping, Reinhard

Deptuła, Milena

Derer, Stefanie

Dergunov, Sergey

Deriu, Marco Agostino

Derkach, Kira V.

Derkinderen, Pascal

Derler, Isabella

Derwich, Katarzyna

Desai, Amar P.

Desai, Amrita

Desai, Chandni

Desai, Moreshwar

Desai, Umesh R.

Desaubry, Laurent

Desbois, Andrew P.

Descamps, Géraldine

Deschanels, Xavier

Desforges, Marc

Deshayes, Frédérique 
Deshpande, Shayu

Desiderio, Vincenzo

Deslouches, Berthony

Desnos, Thierry

Despotovic, Ines

Desriac, Florie

DesRochers, Annie

Dessein, Alain

Desselberger, Ulrich

Dettin, Monica

Dettmer, Ulf

Deuis, Jennifer

Deutsch, Alexander

Deutsch, Melanie

Devarajan, Priyadharshini

Devesa, Jesús

Devillers, James

Devine, Patrick J.

Devireddy, Amith R.

Devroye, Céline

Devy, Jerome

Dey, Kamol

Dey, Madhusudan

Dey, Nandini

Dey, Priyankar

Deyev, Igor E.

Deyev, Sergey M.

Dhakal, Dipesh

Dhakar, Nilesh Kumar

Dhakshinamoorthy, Amarajothi

Dhanasekaran, Murali

Dhanasiri, Anusha

Dhankher, Om Parkash

Dhasmana, Anupam

Dhindsa, Sandeep

Dhooge, Ingeborg Johanna Maria

Dhungel, Bijay

Di Agostino, Silvia

Di Bernardo, Giovanni

Di Bussolo, Valeria

Di Cesare Mannelli, Lorenzo

Di Donato, Marzia

Di Fazio, Pietro

Di Filippo, Ester Sara

Di Filippo, Marzia

Di Fiore, Anna

Di Franco, Simone

Di Gennaro, Francesco

Di Giaimo, Rossella

Di Giannatale, Angela

Di Gioia, Maria Luisa
Di Giovanni, Giuseppe

Di Giulio, Camilio

Di Liegro, Italia

Di Lorenzo, Flaviana

Di Lorenzo, Francesco

Di Maro, Antimo

Di Martino, Orsola

Di Matteo, Adele

Di Matteo, Sabina

Di Mauro, Maria

Di Meo, Ivano

Di Mise, Annarita

Di Nardo, Fabio

Di Natale, Concetta

Di Nisio, Andrea

Di Paola, Rosanna

Di Pasqua, Laura Giuseppina

Di Pietro, Lorena

Di Pietro, Paola

Di Pizio, Antonella

Di Polo, Adriana

Di Pompo, Gemma

Di Resta, Chiara

Di Sansebastiano, Gian-Pietro

Di Serio, Francesco

Di Sotto, Antonella

Di Tucci, Chiara

Di Virgilio, Francisco

Di Zazzo, Erika

Diaconeasa, Zorita

Diaferia, Carlo

Diamantopoulou, Panagiota

Dias, Albino Alves

Dias, Daniel A.

Dias-Ferreira, João

Diaz Hernandez, Miguel

Díaz, Esperanza

Diaz, Eva C.

Diaz, Francisca

Diaz, Marvin

Díaz-Encarnación, Montserrat

Diaz-Garcia, Luis

Diaz-Munoz, Manuel D

Diaz-Rodriguez, Patricia

DiBella, Calogero

DiCarlo, Andrea L.

Diccianni, Mitchell

Dickinson, Bryan C.

Dickson, Benjamin D.

Didiášová, Miroslava

Didier Germain, Arnaud 
Didier, Christine

Didion, Sean P.

Diederichs, Sven

Diego, La Mendola

Diehl, Linda

Diehnelt, Chris W.

Diekwisch, Thomas G.H.

Diene, Seydina

Dienes, Beatrix

Diepold, Andreas

Dietrich, Alexander

Dietrich, Olaf

Diez-Escudero, Anna

Digiacomo, Maria

Dignon, Gregory

Diharce, Julien

Dijk, Aalt

Dijk, Frederike

Dijkhuizen, Lubbert

Dijkstra, Bauke W.

Dika, Emi

Dikalov, Sergey

Dikalova, Anna

Dileep, Vishnu

Dilley, Rodney

Dillman, Adler

Dillman, Adler R.

DIMA, Stefan-Ovidiu

Dimaki, Maria

Dimarogona, Maria

DiMattia, Gabriel

Dimberg, Anna

Dimienescu, Oana Gabriela

Dimitriadis, Stavros

Dimitrios, Fessas

Dimitroff, Charles J.

Dimitroulas, Theodoros

Dimitrov, Eugene

Dimova, Tanya

Dinca, Valentina

Dinel, Anne-Laure

Dinescu, Sorina

Ding, Lin

Ding, Ling-Wen

Ding, Qingbao

Ding, Yaping

Ding, Zhaojun

Dingley, Andrew J.

Dinh, Dzung H.

Dinh, Quynh Nhu

Dinis, Mário
Dinkova, Tzvetanka D.

Dinos, Georgios

Dion, Patrick A.

Dir, Jan

Diretto, Gianfranco

Dirsch, Verena

Disanza, Andrea

Disney, Matthew D.

Dissanayaka, Waruna

Dissel, Stephane

Distasi, Carla

Dite, Toby

Ditengou, Franck

Ditrich, Tomáš

Divashuk, Mikhail

Divella, Rosa

DiVerdi, Joseph

Divi, Rao L.

Divoký, Vladimír

Dixit, Mudit

Dixit, Naveen

Dixon, Brian

Dizdaroglu, Miral

Djavaheri-Mergny, Mojgan

Djerada, Zoubir

Dkhar, Hedwin Kitdorlang

Dmitriev, Alexey A.

Do, Duy Ngoc

Do, Minh Truong

Do, Sun Hee

Dobens, Leonard L.

Doboszewska, Urszula

Dobrev, Ivo

Dobrikova, Anelia

Dobrowolska, Grażyna

Dobrowolski, Piotr

Dobruch, Jakub

Dobrzyńska, Malgorzata

Docampo, Roberto

Docea, Anca Oana

Doci, Colleen

Docimo, Teresa

Docoslis, Aris

Doda, Sai Reddy

Dodd, Rebecca D.

Doddapattar, Prakash

Dodig, Dejan

Doglio, Alain

Dogra, Pranay

Doi, Kent

Doi, Kentaro 
Dokudovskaya, Svetlana

Doles, Jason

Doležal, Karel

Dolezel, Jaroslav

Dolga, Amalia

Dolganov, Pavel Vladimirovich

Dolgikh, Elena A.

Dolinsky, Vernon W.

Dołowy, Krzysztof

Dołowy, Małgorzata

Domanskyi, Andrii

Domaradzki, Piotr

Dombrovsky, Aviv

Dombrowski, Yvonne

Domenech, Maribella

Domenici, Maria Rosaria

Domercq, María

Domingo, Concha

Domingo-Calap, Pilar

Domínguez Díaz, Laura

Domínguez Medina, Eduardo

Domínguez Rebolledo, Álvaro Efrén

Domínguez-Álvarez, Enrique

Domínguez-Vías, Germán

Domoki, Ferenc

Domonkos, Ildikó

Domozych, David S.

Don, Anthony

Donadel, Giulia

Donaldson, Logan W.F.

Donat, Cornelius

Donati Zeppa, Sabrina

Donati, Benedetta

Donati, Giacomo

Donato, Luigi

Donato, Rosario Francesco

Dong, Feng

Dong, Fengping

Dong, L. (Lemeng)

Dong, Mingxin

Dong, Shen

Dong, Yan

Dongiovanni, Paola

Donnay, Isabelle

Donnelly, Callum George

Donnelly, Deirdre

Donnini, Sandra

Donohue, Maura

Donzelli, Elisabetta

Donze-Reiner, Teresa

Doody, Gina
Doppler, Stefanie

Döppner, Thorsten R.

Dores, Michael R.

Doretto, Paolo

Dorfman, Ruslan

Döring, Yvonne

Dormán, György

Dorn, Alexander

Doroftei, Bogdan

Dorotea, Muck-Seler

Dorozhkin, Sergey V.

Dorożyński, Przemysław

Dorsky, Richard

Dos Anjos Pires, Maria

Dos Santos, João Miguel Marques

Dos Santos, Nuno Rodrigues

Dos Santos, Wagner

DOSOKY, NOURA

Doss, Mohan

Dosset, Megalie

Dou, Qingping

Dou, Xiaoguang

Doud, Michael

Doulamis, Ilias

Douros, Antonios

Doustkhah Heragh, Esmaeil

Doveston, Richard

Dovizio, Melania

Dovnik, Andraz

Doxakis, E.

Doyle, Siamsa

Dozois, Charles

Drabczyk, Anna

Dracatos, Peter Michael

Draganova, Milena

Dragavon, Joseph M.

Dragomir, Mihnea

Dragoni, Silvia

Dragos, Horvath

Drake, Penelope M.

Drakos, Elias

Dramsi, Shaynoor

Dreher, Mark L.

Dreier, Rita

Dreschers, Stephan

Drexler, Berthold

Drey, Michael

Drgan, Viktor

Dridi, Sami

Drissi-Habti, Monssef

Drochioiu, Gabi 
Drosten, Matthias

Drozd, Marcin

Drozdov, Anatoliy

Droździk, Marek

Drulis-Fajdasz, Dominika

Drummer, Heidi

Druzbicki, Kacper

Drużyńska, Beata

Drzazga, Michał

Drzazga, Zofia

Drzymała-Czyż, Sławomira

Du, Qian

Du, Xiaojun

$\mathrm{Du}$, Zhifeng

Duan, Jialei

Duan, Zhijun

Duarte, Ana

Duarte, Filipe V.

Duarte, Joao

Duarte, Maria Cristina

Dubash, Taronish Dorab

Dubbelboer, Ilse

Dubey, Ramin

Dubiel, Wolfgang

Dubin, Grzegorz

Dubinin, Mikhail V.

Dubińska-Magiera, Magda

DuBois, Robert

Dubreucq, Bertrand

Dubrez, Laurence

Dubrova, Yuri

Dubrovina, Alexandra

Duchi, Serena

Ducreux, Sylvie

Ducza, Eszter

Duda, Katarzyna

Dudas, Jozsef

Dudás, Zoltán

Duddy, William

Dudka, Jaroslaw

Dudok, Jeroen

Duechler, Markus

Duennwald, Martin L

Dufossé, Laurent

Dugar, Rohit P.

Dugé De Bernonville, Thomas

Duguez, Stephanie

Duhan, Vikas

Duitman, JanWillem

Dulermo, Rémi

Dulskas, Audrius
Duma, Luminita

Dumas, Philippe

Dumaz, Nicolas

Dumez, Sylvain

Dumitru, Gabriela

Dumnicka, Paulina

Dunaeva, Marina V.

Dunbar, Gary L.

Dungan, Cory

Dunham, Rex A.

Dunić, Jasenka Antunović

Dunlap, Christopher

Dunlop, Elaine

Dunlop, Katharine

Dunphy, Michael J.

Dünser, Kai Alexander

Duplaa, Cecile

Duployez, Nicolas

Dupraz, Sebastian

Durand, Béatrice C.

Durand, Nisha

Duran-Ortiz, Silvana

Duranti, Marcello

Duranton, Christophe

Durazzo, Marilena

Durbeej, Madeleine

Durgo, Ksenija

Durham, Benjamin H.

Durian, Guido

Duriancik, David

Duric, Vanja

Durocher, Francine

Durrant, Lindy G.

Dürst, Matthias

Dursun, Serdar

Duryee, Michael J.

Dusi, Veronica

Düsterhöft, Stefan

Dustin, Lynn

Dutartre, Patrick

Duterque-Coquillaud, Martine

Dutot, Mélody

Dutt, Arun K.

Dutta, Arijit

Dutta, Rajesh

Dutta, Sanjucta

Dutto, Moreno

Duvigneau, J. Catharina

Duvnjak, Tomislav

Duwaerts, Caroline

Düzgünes, Nejat 
Dvorak, Cheryl

Dvorakova, Magdalena Chottova

Dvorakova-Hortova, Katerina

Dworkin, Sebastian

Dyakin, Victor V.

Dyda, Fred

Dye, Danielle E.

Dykhuizen, Emily

Dymarska, Monika

Dymińska, Lucyna

Dynarowicz-Latka, Patrycja

Dyson, Paul J.

Dzantiev, Boris

Dzhimak, Stepan

Dzidic, Alen

Dziedzic, Arkadiusz

Dzięgiel, Piotr

Dziegielewska, Barbara

Dziendzikowska, Katarzyna

Dzietko, Mark

Dziewięcka, Ewa

Dzimitrowicz, Anna

Dziurka, Kinga

Dzobo, Kevin

Eamens, Andrew L.

Ear, Jason

Earl, Julie

Earle, Kenneth A.

Easwaran, Hariharan

Eaton, Douglas

Eaton, Peter

Ebeed, Heba

Eberhardt, Mirjam Jeanette

Eberlein, Bernadette

Ebersviller, Seth

Ebert, Timothy A

Ebihara, Lisa

Eble, Johannes

Ebrahimi, Aziz

Eccles, David

Echchannaoui, Hakim

Echevarria, Miriam

Echigoya, Yusuke

Ecke, Thorsten Holger

Eckert, Alexander W.

Eckert, Alexaner

Eckl, Katja

Eckschlager, Tomas

Economou, Anastassios

Eda, Shigetoshi

Edamakanti, Chandrakanth R
Edeas, Marvin

Edelman, Irina $S$.

Eder, Iris E.

Edin, Nina Jeppesen

Ediriweera, Meran Keshawa

Edman, Maria

Edmé, Serge

Edskes, Herman K.

Edwards, Adam B.

Eferl, Robert

Eftestøl, Einar

Egan, Terrance

Egawa, Kiyoshi

Egbert, Jeremy R.

Egea Gutiérrez-Cortines, Marcos

Egea, Gustavo

Egea, Isabel

Egerszegi, István

Egger, Boris

Eggers, Christian $\mathrm{H}$

Egorova, Ksenia

Ehlen, J. Christopher

Ehler, Elisabeth

Ehrich, Marion

Eichmeier, Ales

Eichstaedt, Christina

Eikmans, Michael

Eilertsen, Karl-Erik

Eiring, Anna

Eisendle, Klaus

Eisenstat, David D.

Eisinger-Mathason, Tzipora S.Karin

Eiz-Vesper, Britta

Ekberg, Jenny

Ekdahl, Kristina N.

Ekker, Marc

EkVitorin, Jose

El Kasmi, Farid

El Mourabit, Haquima

El Shamieh, Said

Elango, Jeevithan

Elansary, Hosam O.

Elaswad, Ahmed

Elder, Rhoderick

Eleftheriadis, Theodoros

Elemans, J.A.A.W.

El-Esawi, Mohamed A.

Elewa, Yaser H.A.

Elezkurtaj, Sefer

Elfalleh, Walid

Elferink, Cornelis 


\author{
Elhai, Muriel \\ Elia, Maurizio \\ Eliaz, Isaac \\ Elida Nora, Ferri \\ Eligini, Sonia \\ Elis, Sebastien \\ Elisashvili, Vladimir \\ Eliseev, Roman \\ Eljaafari, Assia \\ El-Jawhari, Jehan \\ Elkabets, Moshe \\ Elleder, Daniel \\ Ellert-Miklaszewska, Aleksandra \\ Ellery, Stacey J. \\ Elliott, Evan \\ Ellis, Cameron \\ Ellis, Jessica M. \\ Ellison, Michael \\ Ellwood, Simon R. \\ ElMallah, Mai K. \\ Elmarakby, Ahmed \\ El-Mashtoly, Samir F. \\ Elmouki, Ilias \\ Elnitski, Laura \\ Elsea, Sarah H. \\ El-Seedi, Hesham \\ Elshabrawy, Hatem \\ Elshafie, Hazem S. \\ El-Sharkawy, Islam \\ Elson, Ari \\ Elviri, Lisa \\ Elyaman, Wassim \\ Emami, Payam \\ Emous, M. \\ Emri, Eszter \\ Emrich, Fabian C. \\ Endepols, Heike \\ Endo, Makoto \\ Endo, Mitsuharu \\ Endo, Mizuki \\ Endre Károly, Kristóf \\ Endres, Kristina \\ Ene, Razvan \\ Eng, Lo \\ Engedal, Nikolai \\ Engel, Kathrin \\ Engel, Stanislav \\ Engeland, Christine E. \\ Engelberg, David \\ Engelberth, Jurgen \\ Engelhardt, Paul Friedrich
}

Engelmann, Ilka

Engelmann, Peter

Engler, Anna

Enguita, Francisco J.

Enjeti, Anoop

Ennequin, Gael

Enomoto, Atsushi

Enomoto, Hirayuki

Entelis, Nina

Enyedi, Agnes

Eom, Gwang Hyeon

Epand, Richard

Eppard, Elisabeth

Epping, Eric A.

Eppler, Elisabeth

Eramo, Stefano

Eraña, Hasier

Erastova, Valentina

Erben, Philipp

Erdélyi, Miklós

Erdmann, Kati

Erdődi, Ferenc

Erenpreisa, Jekaterina

Eri, S. Srivatsan

Eric Kast, Richard

Eric, Tillman

Erickson, Michelle A.

Erickson, Timothy

Eriksen, Erik F.

Erjavec, Gordana Nedic

Erland, Lauren

Ermakov, Artem

Ermolaeva, Svetlana

Ernsberger, Paul

Ernst Seemann, Stefan

Ernst, Margot

Erogbogbo, Folarin

Ersilia, Alexa

Erskine, Lynda

Erwin, William Mark

Esakimuthu, Shankar

Escalante, Ricardo

Escalona, Hermes E.

Escargueil, Alexandre

Escobedo Monge, Marlene Fabiola

Escors, David

Escoté, Xavier

Escribese, María M.

Escriu, Fernando

Eskelinen, Eeva-Liisa

Eskiw, Christopher $\mathrm{H}$. 
Eslami, Hossein

Espada, Jesús

Espadas Villanueva, Isabel

Espen, Luca

Espinós, Carmen

Espinosa-Diez, Cristina

Espinosa-Garcia, Claudia

Espinoza-Fonseca, Lennane Michel

Espona-Noguera, Albert

Esposito, Emilio Xavier

Esposito, Francesco

Esposito, Gabriella

Esposito, Maria Teresa

Esposito, Roberta

Esposito, Sergio

Essa, Musthafa

Esser, Charlotte

Esser, Michael J.

Essner, Jeff

Esteban, Vanesa

Estévez, Francisco

Estévez, Jorge

Estévez, Laura García

Estrada-Gutierrez, Guadalupe

Esworthy, Robert Steven

Etich, Julia

Etienne-Manneville, Sandrine

Etsushi, Kuroda

Ettayapuram Ramaprasad, Azhagiya

Singam

Eudald, Casals

Eufemi, Margherita

Eugène, MEGNASSAN

Eugenin, Eliseo

Euler, Gerhild

Eun, Jung Woo

Euverink, Gert-Jan

Éva, Mikó

Evangelisti, Camilla

Evangelisti, Claudio

Evangelisti, Edouard

Evangelopoulos, Michael

Evani, Shankar Jaikishan

Evans, Christopher $\mathrm{H}$.

Evans, Dolores Gareth R.

Evans, Iwan Robert

Eveillard, Marion

Even-Hernandez, Pascale

Evert, Matthias

Evinger, Marian

Ewans, Matthew
Ewing, Adam

Exbrayat, Jean-Marie

Eyers, Patrick

Eysel-Gosepath, Katrin

Eysteinsson, Thor

Ezquer, Ignacio

Ezura, Yoichi

Ezzeddine, Zeinab

Fabbian, Fabio

Fabbrini, Maria Serena

Fabbrocini, Gabriella

Fabi, João Paulo

Fabian, Zsolt

Fabiani, Roberto

Fabiano, Eduardo

Fabio, Munari

Fabiszewska, Agata

Fabrizio, Federico Pio

Facchiano, Angelo

Facchin, Federica

Facchinetti, Roberta

Facchini, Gaetano

Faccio, Greta

Faciotti, Federica

Facoetti, Angelica

Fadeeva, Irina

Fafilek, Bohumil

Fagerholm, Susanna C.

Faggioli, Francesco

Faggioni, Michela

Fagioli, Franca

Fagoonee, Sharmila

Fahlbusch, Fabian B.

Fahnestock, Margaret

Failla, Cristina M.

Failla, Osvaldo

Faille, Christine

Fairless, Richard

Fairweather, Stephen

Fais, Antonella

Faivre-Sarrailh, Catherine

Fajardo, Val Andrew

Fakhouri, Walid D.

Fakhrullin, Rawil F.

Falcieri, Elisabetta

Falcone, Italia

Faleiro, Maria Leonor

Falguières, Thomas

Fali, Tinhinane

Falistocco, Egizia 
Falkinham III, Joseph O.

Fallini, Claudia

Falls, Zackary

Falsini, Benedetto

Fambrini, Massimiliano

Famulski, Jakub

Fan, Guo-Chang

Fan, Hueng-Chuen

Fan, Jianglin

Fan, Lusheng

Fan, Wenchun

Fan, Zheng

Fan, Zhichao

Fandrey, Joachim

Fane, Mitchell

Fanelli, Valentina

Fang, Cheng

Fang, Evandro F.

Fang, Jennifer

Fang, Jiyu

Fang, Junnan

Fang, Qingming

Fang, Runchen

Fang, SungSoon

Fang, Xing

Fang, Yi

Fanning, Saranna

Fanourakis, Galinos

Fantacuzzi, Marialuigia

Fantini, Massimo

Fantini, Sebastian

Faouzi, Malika

Farabegoli, Fulvia

Faraji, Farhoud

Faralli, Jennifer A.

Faraoni, Isabella

Farcasanu, Ileana C.

Farè, Silvia

Fareed, Amber

Fareed, Jawed

Faria De Oliveira, VirusMichelli

Faridi, Pouya

Fariña, José B.

Farina, Nicholas

Farinha, Carlos

Faris, Robert

Farjon, Jonathan

Farkas, Attila E.

Farkas, Laszlo

Farkaš, Vladimír

Faron-Górecka, Agata
Farrar, Michael A.

Fasinu, Pius

Fasler, Elizaveta

Fasolato, Cristina

Fass, Deborah

Fast, Loren D.

Fatkhudinov, Timur

Faussone-Pellegrini, Maria Simonetta

Faustino, Randolph

Faux, David

Favaloro, Emmanuel

Favara, Giuliana

Favery, Bruno

Favi, Evaldo

Favia, Maria

Favier, Anne-Laure

Favre, Claudio

Fay, Fabienne

Fazeli, Alireza

Fazi, Francesco

Fazio, Francesco

Feás, Xesús

Febbraio, Ferdinando

Fede, Caterina

Fedele, Francesco

Fedele, Monica

Federico, Antonio

Fedorova, Olga S.

Fedorova, Olga V.

Fedotova, Julia O.

Feghali-Bostwick, Carol

Feher, Gergely

Feher, Tamas

Fehon, Richard

Fehr, Anthony

Fehse, Boris

Feinn, Richard S.

Felder-Schmittbuhl, Marie-Paule

Feldman, Steve

Feldo, Marcin

Felicetti, Tommaso

Feliciano, Joana

Félix, Carina

Félix, Luis

Felley-Bosco, Emanuela

Felmerer, Gunther

Felten, Matthias

Fendt, Markus

Feng, Chaohui

Feng, Hua-Jun

Feng, Huapeng 
Feng, Lijuan

Feng, Yongjun

Fenini, Gabriele

Fenton, Tim

Feo, Francesco

Feofanov, Alexey V.

Fercher, Christian

Feregrino-Pérez, Ana A.

Ferens-Sieczkowska, Mirosława

Ferji, Khalid

Fernandes Fagundes, Nathalia

Fernandes, Ana

Fernandes, Hugo

Fernandes, Maria Helena

Fernandes, Maria José Da Silva

Fernandes, Mónica

Fernandes, Tiago

Fernandez Fairén, Mariano

Fernandez, Agustin F.

Fernandez, Diana

Fernández, Ignacio

Fernández, José A.

Fernández, Lucía

Fernández, Manuel

Fernandez, Mercedes

Fernandez, Paula

Fernandez, Sandra V.

Fernández, Vanesa Martín

Fernández-Álvarez, Alfonso

Fernandez-Botran, Rafael

Fernandez-Funez, Pedro

Fernández-García, Marta

Fernández-Gutiérrez, Mar

Fernández-Mateos, M. Pilar

Fernandez-Pozo, Noe

Fernández-Rodríguez, Javier

Fernandez-Rojo, Manuel A.

Fernández-Ruiz, Javier

Fernández-Sánchez, Laura

Fernández-Tomé, Samuel

Fernández-Velasco, María

Fernandez-Vizarra, Erika

Fernando, Binosha

Fernando, M. Rohan

Ferramosca, Alessandra

Ferrando, Riccardo

Ferrante, Antonella

Ferrante, Antonio

Ferrante, Tony

Ferrara, Felicetto

Ferrara, Nicola
Ferrara, Nicole C.

Ferrari, Federico

Ferrari, Paola

Ferrario-Méry, Sylvie

Ferraroni, Marta

Ferrarotti, Francesco

Ferraz, Marcia De Almeida Monteiro Melo

Ferraz, Ricardo

Ferreira, Helena

Ferreira, Jorge

Ferreira, Leonardo M.R.

Ferreira, Manuel M.

Ferreira, Nelson

Ferreira, Paula M.

Ferreira, Samira

Ferreira-Cerca, Sebastien

Ferreira-Dias, Graça M.

Ferreon, Allan Chris M.

Ferreon, Josephine

Ferrer, Gerardo

Ferreras, José Miguel

Ferreri, Carla

Ferrer-Miralles, Neus

Ferrer-Montiel, Antonio

Ferrer-Perez, Carmen

Ferretta, Anna

Ferri, Flaminia

Ferrigo, Davide

Ferriz-Martínez, Roberto Augusto

Ferro, Albert

Ferro, Domenico

Ferro, Matteo

Ferro, Riccardo

Ferroni, Lorenzo

Ferruz-Capapey, Noelia

Fessner, Wolf-Dieter

Fetissov, Sergueï O.

Fettke, Jörg

Fiandra, Luisa

Fiani, Maria Luisa

Fiaschi, Tania

Fields, Peter D.

Fierabracci, Alessandra

Fiering, Steve N.

Fiering, Steven N.

Fieseler, Lars

Figaj, Donata

Figiel, Adam

Figueiredo, Andreia

Figueiredo, Constança

Fijak, Monika 
Fik-Jaskółka, Marta A.

Filaci, Gilberto

Filadi, Riccardo

Filarowski, Aleksander

Filek, Maria

Filella, Xavier

Filgueira, Carly

Filho, Alves

Filiou, Michaela

Filipcik, Peter

Filipek, Agnieszka

Filipek, Anna

Filipič, Bratko

Filipkowski, Robert

Filipova, Inese

Filipovic, Milos

Filippelli, Amelia

Filippou, Panagiota S.

Filippov, Andrei

Filippov, Mikhail A.

Filip-Psurska, Beata

Filiz, Ertugrul

Fillmore, Helen

Filová, Eva

Fimognari, Carmela

Finelli, Carmine

Fineschi, Vittorio

Finet, Cédric

Finetti-Sialer, Mariella Matilde

Fingerhut, Ralph

Fini, Mehdi A

Fink, Dieter

Fink, George

Finkelstein, David

Finlay-Schultz, Jessica

Finlayson, Courtney Anne

Fintini, Danilo

Fiocchetti, Marco

Fionda, Cinzia

Fioramonti, Xavier

Fiore, Michele

Fiorelli, Susanna

Fiorentini, Giammaria

Fiorentino, Gabriella

Fiorenza, Roberto

Fiori, Enrico

Fiori, Mariana

Fiorillo, Luca

Fiorio Pla, Alessandra

Firdessa, Rebuma

Firestein, Bonnie
Fisar, Zdenek

Fischer, Dagmar-Christiane

Fischer, Jörg

Fischer, Lukáš

Fischer-Fodor, Eva

Fischer-Smith, Tracy

Fiscon, Giulia

Fisher, Jonathan S.

Fisher, Scott

Fisher-Wellman, Kelsey

Fishman, Pnina

Fisslthaler, Beate

Fiszer, Agnieszka

Fitches, Elaine

Fitton, Helen

Fitz, Nicholas F.

Fiume, Giuseppe

Fladung, Matthias

Flak, Jonathan N

Flannery, John

Fleites, Laura A.

Fletcher, Anne

Fletcher, Madeleine M.

Fletcher, Nicola F.

Fletcher, Susan

Flick, Matthew

Fliefel, Riham M.

Fliegel, Larry

Flieger, Jolanta

Fliegert, Ralf

Flinn, Barry

Flint, Mike

Flint, Steve

Florán, Benjamín

Florczyk, Stephanie

Florea, Adrian

Florea, Ana-Maria

Florescu, Monica

Flores-Félix, J.D.

Flores-Guerrero, Jose L.

Flores-López, María L.

Floria, Mariana

Florian, Maria Carolina

Floris, Matteo

Flors, Victor

Flottmann, Fabian

Fluri, Felix

Fodor, Ferenc

Foecking, Melanie

Fogolari, Federico

Fojta, Miroslav 
Fojtová, Miloslava

Foka, Pelagia

Fokkema, M. Rebecca Heiner

Fol, Marek

Foley, Ann C.

Foley, Chris

Folgosa, Filipe

Folini, Marco

Fologea, Daniel

Fonkem, Ekokobe

Fonseca, Bruno Miguel Reis Da

Fontana, Barbara D.

Fontana, Federico

Fontana, Paolo

Fontanesi, Flavia

Fontenla, Jose Angel

Foong, Jaime

Forbes, Louisa

Forbes-Hernández, Tamara Y.

Forbester, Jessica

Ford, Nicole

Forero-Castro, Maribel

Foret, František

Forini, Francesca S.

Forlino, Antonella

Forma, Ewa

Formanowicz, Dorota

Formela, Krzysztof

Formoso-Rafferty, Nora

Fornai, Francesco

Fornai, Matteo

Fornstedt, Torgny

Forster, Jameson

Forstova, Jitka

Forsyth, Christopher B.

Forsyth, Nicholas

Fort, Patrice E.

Fort, Philippe

Forte, Iris Maria

Forte, Nicola

Forte, Stefano Forte

Fortis, Sotirios P.

Fortunato, Orazio

Foster, John

Foster, Tim

Foster, Toshana Lauria

Foster, Warren G.

Fotouhi, Omid

Fouladkhah, Aliyar

Foulke-Abel, Jennifer D.

Foulquier, Sébastien
Fountain, Samuel J.

Fountoulaki, Eleni

Fournier, Antoine

Fournier, Marcia V.

Fournier, Neil M.

Fowkes, Rob

Fowler, Marcie

Fox, Julie

Fox, Keith R.

Foyer, Christine $\mathrm{H}$

Fracanzani, Anna

Frachisse, Jean-Marie

Fradet, Vincent

Fradin, Chantal

Fradin, Delphine

Fraga-Corral, Maria

Fragai, Marco

Fragliasso, Valentina

Fraguas-Sánchez, Ana Isabel

Fraichard, Stéphane

Fraineau, Sylvain

Fraizer, Gail

Frampton, Garrett

Francesca, Lunardi

Franceschelli, Sara

Francesco, Scavello

Francesco, Trapasso

Francis, Ashwanth Christopher

Francis, Brian R.

Francis, Hane

Francis-Boyle, Olivia

Francisco, Vera

Franco, Irene

Franco, Luis

Franco, Marina Santiago

Franco, Omar

Franco, Rodrigo

Franco-Barraza, Janusz

Francs-Small, Catherine Colas Des

Frandes, Mirela

Frank, Luiza

Frank, Saša

Franke, Jakob

Frank-Kamenetskaya, Olga V.

Franko, Andras

Franks, Jennifer M.

Franzè, Annamaria

Franzese, Monica

Franzini, Anca

Franzyk, Henrik

Frasca, Angelisa 
Frasca, Loredana

Fraser, Michael

Frassanito, Maria Antonia

Frasson, Ilaria

Frati, Alessandro

Fratzl-Zelman, Nadja

Frazer, Alan

Frazer, J. Kimble

Frech, Moritz

Frederick Charles, Nucifora

Fredholm, Merete

Fredua-Agyeman, Rudolph

Freedman, Benjamin

Freedman, Jonathan

Freiberger, Sandra Nicole

Freichel, Marc

Freitag, Julien

Freitas De Freitas, Lucas

Freitas, Renata

Freitas, Vanessa Morais

Frenkel, Amit

Frenkiel, Saul

Freude, Kristine

Freudlsperger, Christian

Freytag, Saskia

Frezza, Claudio

Frezzato, Federica

Frias Casas, Mario

Frick, Manfred

Fricker, Lloyd D.

Fried, Miklos

Frieden, Maud

Frieri, Giuseppe

Fries, Fabian

Frietze, Seth

Frigeri, Antonio

Friis-Hansen, Lennart

Frisch, Steven M.

Frischauf, Irene

Frisso, Giulia

Fritsche, Raphaela

Frittitta, Lucia

Froehlich, Leopold

Froeyen, Matheus

Froeyen, Mathy

Frohme, Marcus

Frolíková, Michaela

Frosini, Maria

Frossi, Barbara

Fruchon, Séverine

Frugé, Andrew
Fruhbeck, Gema

Fry, Stephen C.

$\mathrm{Fu}$, Jianming

$\mathrm{Fu}$, Lianwu

$\mathrm{Fu}$, Xing

$\mathrm{Fu}$, Xuewei

$\mathrm{Fu}$, Ying

$\mathrm{Fu}$, Zheng

$\mathrm{Fu}$, Zhongjie

Fuchs, Dietmar

Fuchs, Ota

Fucile, Sergio

Fuellen, Georg

Fuentes, Eduardo

Fuggi, Amodio

Fujihara, Yoshitaka

Fujihara, Yuko

Fujii, Hideaki

Fujii, Hideki

Fujii, Hiroaki

Fujii, Kentaro

Fujii, Masayuki

Fujii, Sho

Fujii, Yoshiharu

Fujii, Yuki

Fujimori, Ko

Fujimoto, Ayataka

Fujinaga, Koh

Fujisawa, Yasuhiro

Fujita, Kaori

Fujita, Kazutoshi

Fujita, Koji

Fujita, Masaki

Fujita, Mikako

Fujita, Ryosuke

Fujita, Yuki

Fujiwara, Keigi

Fujiwara, Satoru

Fujiwara, Tomoya

Fujiwara, Toshinobu

Fujiwara, Yasuhiro

Fukada, So-ichiro

Fukagawa, Tatsuo

Fukai, Tohru

Fukuchi, Satoshi

Fukuda, Aisaku

Fukuda, Mitsuru

Fukuda, Noboru

Fukuda, Takeshi

Fukudome, Akihito

Fukuhara, Atsunori 
Fukuhara, Yasuyuki

Fukunaga, Kohji

Fukunaga, Naoto

Fukunaga, Ryuya

Fukunaga, Tsukasa

Fukuoka, Hidenori

Fukushima, Atsushi

Fukushima, Hiroshi

Fukushima, Kentaro

Fukushima, Tsuyoshi

Fukuta, Shiro

Fukuyama, Tomoki

Fulci, Valerio

Fulciniti, Franco

Fulford, Tom

Fulle, Stefania

Fuller, Bryan

Fuller, Maria

Fumagalli, Francesca

Fumagalli, Laura

Fumarola, Claudia

Funakoshi, Kengo

Funato, Noriko

Funato, Yosuke

Funel, Niccola

Funk, Richard

Furdui, Bianca

Füredi, András

Furfaro, Anna Lisa

Furtula, Boris

Furue, Masutaka

Furuta, Kazuyuki

Furuta, Saori

Fushida, Sachio

Füssel, Susanne

Fuster, Jose Javier

Futakuchi, Mitsuru

Futoma-Kołoch, Bożena

Futrega, Kathryn

Futter, Clare

Fuu-Jen, Tsai

Gaag, Kristiaan J. Van Der

Gabandé-Rodríguez, Enrique

Gabbia, Daniela

Gabbiani, Giulio

Gabdulkhakov, Azat

Gaber, Timo

Gabernet, Gisela

Gabler, Christoph

Gaborit, Bénédicte

Gabrielli, Brian
Gabrielson, Nathan P.

Gaceb, Abderahim

Gacesa, Ranko

Gachomo, Emma

Gachon, Frederic

Gackowski, Daniel

Gaczynska, Maria

Gad, Ahmed

Gadad, Shrikanth

Gadaleta, Agata

Gadeau, Alain-Pierre

Gadella, Theodorus WJ

Gaetani, Lorenzo

Gafencu, Anca

Gaffet, Eric

Gaffney, Christopher

Gaforio, José

Gafurov, Marat

Gagat, Maciej

Gaglia, Marta Maria

Gagliano, Caterina

Gagliano, Nicoletta

Gagliardi, Paolo Armando

Gaglio, Daniela

Gagniuc, Paul

Gago-Fuentes, Raquel

Gai, Zhibo

Gaid, Mariam

Gaida, Matthias

Gaiddon, Christian

Gaipl, Udo S.

Gaitonde, Vishwanath

Gajavelli, Shyam

Gajdosik, Martina Srajer

Gajdzis, Pawel

Gajger, Ivana Tlak

Gajos-Michniewicz, Anna

Galan, Jacob

Galan, Maria

Galasso, Giovanni

Galateanu, Bianca

Galatou, Eleftheria

Galazios, Georgios

Galderisi, Umberto

Galdiero, Marilena

Galeffi, Patrizia

Gales, Luís

Gali, Himabindu

Galiba, Gabor

Galina, Gencheva

Galindo, Antonio 
Galindo, Fernando Shintate

Galindo-Villegas, Jorge

Galkin, Alexander S.

Galkina, Svetlana I.

Gall, Joseph

Gáll, Tamás

Gáll, Zsolt

Gallagher, Jennifer E. G.

Gallagher, Renata

Gallagher, Travis

Gallavardin, Thibault

Gallego, Juan Carlos

Gallego, Óscar S.

Galleni, Moreno

Gallet, Paul François

Galli, Alvaro

Galliciotti, Giovanna

Gallo Cordova, Alvaro

Gallo, Daniela

Gallo, Maria Pia

Gallo, Salvatore

Gallo, Simona

Gallorini, Marialucia

Galpert, Deborah

Galuska, Sebastian P.

Galuska, Sebastian Peter

Galvagni, Federico

Galvão, Adelino M.

Galvao, Izabela

Gama, Adelina

Gama, Sofia

Gama, Vivian

Gamal El-Din, Tamer M.

Gamaleev, Vladislav

Gaman, Amelia

Gaman, Amelia Maria

Gaman, Mihnea-Alexandru

Gambardella, Alessandro

Gambardella, Jessica

Gambari, Laura

Gamberi, Tania

Gamboa-deBuen, Alicia

Gamella Carballo, Maria

Gamian, Andrzej

Gamper, Armin

Gan, Renyou

Gan, Samuel

Ganaie, Safder

Ganapathy, Vadivel

Ganassi, Sonia

Ganau, Mario
Ganazzoli, Fabio

Gandellini, Paolo

Gandhirajan, Rajesh Kumar

Gandía, Mónica

Ganesan, A.

Ganesan, Murali

Ganesh Rai, Bantukallu

Gangadaran, Prakash

Ganini, Carlo

Ganji, Rakesh

Gannett, Peter M.

Gannon, Maureen

Ganopoulous, Ioannis

Ganser, Christian

Gantz, Stephanie

Ganz, Julia

Gao, Gary

Gao, Jie

Gao, Shiqiang

Gao, Song

Gao, Xiaolong

Gaponenko, Vadim

Garaigorta, Urtzi

Garanto, Alejandro

Garanto, Alex

Garau, Alessandra

Garavelli, Livia

Garbar, Christian

Garcia Alonso, Maria Alejandra

Garcia De Frutos, Pablo

Garcia Perez, Samuel

García Souto, Daniel

Garcia, Andres Sanz

Garcia, Antonio

García, Concepción

Garcia, Cyrielle

García, Gabriela

Garcia, Jose Martin

García, María-Luz

Garcia, Michelle

García, Montserrat

Garcia, Natalia Mora

Garcia, Samuel

Garcia, Victor

García-Álvarez, Yolanda

García-Aranda, Marilina

García-Barrado, María José

Garcia-Borron, Jose Carlos

Garcia-Caballero, Agustin

Garcia-Deibe, Ana

García-Díaz Barriga, Gerardo 
García-Domínguez, Mario

Garcia-Estañ, Luis Perez

Garcia-Fruitos, Elena

Garcia-Gallego, Sandra

García-García, Andrés

García-García, Rosa María

Garcia-Garrido, Jose Manuel

Garcia-Gil, Mercedes

Garcia-Giralt, Natalia

Garcia-Gonzales, Carlos

García-López, Rodrigo

Garcia-Molina, Antoni

Garcia-Orduña, Pilar

García-Otín, Ángel Luis

Garcia-Redondo, Ana Belen

Garcia-Sanchez, Asuncion

García-Sánchez, Susana

García-Sancho, Javier

Garcia-Sherman, Melissa

García-Silva, Susana

Garcia-Sosa, Alfonso

García-Villalón, Angel Luis

Gard, Paul

Gardiner, Amy S

Gardiner, Jason

Gardner, Andrew F.

Gardón, Juan Carlos

Gareri, Pietro

Garg, Koyal

Gargiulo, Valentina

Garí, Eloi

Garikipati, Venkata

Garinis, George

Garino, Cristiano

Garita-Hernandez, Marcela

Garmanchuk, L. V.

Garn, Holger

Garncarek, Zbigniew

Garofalo, Cecilia

Garozzo, Domenico

Garre, Victoriano

Garrett, Scott

Garriga, Pere

Garrity, Deborah

Gartel, Andrei

Garvalov, Boyan K.

Garweg, Justus G.

Garzon, Simone

Garzon-Muvdi, Tomas

Gas, Piotr

Gąsecka, Aleksandra
Gash, Don M

Gashti, Mazeyar Parvinzadeh

Gasic, Ksenija

Gąsior-Perczak, Danuta

Gasiūnas, Giedrius

Gaspar, Krisztian

Gaspar, Maria Manuela

Gáspári, Zoltán

Gasparri, Fabio

Gasparri, Roberto

Gasparski, Alexander

Gasser, Susan M

Gasset, Maria

Gassman, Natalie

Gasstoł, Maciej

Gasull, Xavier

Gaszner, Balázs

Gates, Colin

Gatti, Antonietta

Gatto, Francesca

Gatzka, Martina

Gaubert, Alexandra

Gauda, Estelle

Gaudin, Robert

Gaudio, Agostino

Gautam, Mukesh

Gautam, Prson

Gautheron, Jérémie

Gauthier, Adrien

Gauthier, Benoit

Gauthier, Thierry

Gautier, Mathieu

Gavalas, Anthony

Gavara, Laurent

Gavira, José

Gaviraghi, Marco

Gavriilaki, Eleni

Gawel, Damian

Gawel, Kinga

Gawlik, Aneta

Gawlik-Dziki, Urszula

Gay, Francesca

Gay-Quéheillard, Jerome

Gaze, David C.

Gazerani, Parisa

Gazouli, Maria

Gazzin, Silvia

Gdaniec, Zofia

Ge, Xiaodong

Geary, Sean

Gebert, Johannes 
Gedara, Pradeep

Gee, Christine E

Geest, Ferdy Van

Geetha, Thangiah

Gegotek, Agnieszka

Gehlert, Sebastian

Gehring, Christoph

Geisberger, Roland

Gelissen, Ingrid

Genco, Caroline Attardo

Gendaszewska-Darmach, Edyta

Gendek-Kubiak, Hanna

Genders, Amanda

Geng, Xiaokun

Gennari, Alessandra

Gennari, Luigi

Gennery, Andrew

Genova, Tullio

Genovese, Carlo

Genovese, Tiziana

Gent, Martin

Gentile, Alessandra

Gentile, Carla

Gentile, Luca

Gentile, Pietro

Gentile, Vittorio

Gentilin, Erica

Gentle, Ian E.

Gentry, Matthew S.

Gentzsch, Martina

Geoerger, Birgit

George, Anne

Georgescu, Adriana

Georgiev, Georgi As.

Georgieva, Milena

Georgieva, Radostina

Georgii, Elisabeth

Geppert, Mark

Gera, Ruchi

Geraci, Fabiana

Geraghty, Patrick

Gerasimaitè, Rūta

Gerber, André

Gerbino, Andrea

Gerbst, Alexey G.

Gerding, Heinrich

Gerdol, Marco

Geremia, Silvano

Gereñu, Gorka

Gergs, Ulrich

Gerisch, Günther
Gerli, Mattia

Gerlich, Wolfram

Gerling, Marco

Gerloff, Dennis

Germain, Lucie

Germain, Pierre

German, Nadezhda A.

Germanidis, Georgios

Germano, Giovanni

Germini, Diego

Gerriets, Valerie

Gervois, Pascal

Gescher, Andreas

Gesi, Camilla

Geso, Moshi

Gessani, Sandra

Gessler, Florian

Gessner, Guido

Geta, David A.

Getz, Godfrey

Ghadieh, Hilda E.

Ghai, Vikas

Ghaisas, Shivani

Ghaleh, Bijan

Ghandhi, Shanaz

Ghandour, Rashed

Ghantous, Yasmine

Ghasemi, Rasoul

Ghatak, Arindam

Ghavami, Saeid

Ghayor, Chafik

Ghelli Luserna Di Rorá, Andrea

Gherasim, Carmen

Ghesquiere, Bart

Ghezzi, Daniele

Ghiani, Cristina

Ghiasi, Seyed Mojtaba

Ghidoni, Riccardo

Ghigna, Claudia

Ghigo, Eric

Ghilli, Matteo

Ghinet, Alina

Ghiran, Ionita C.

Ghiroldi, Andrea

Ghislat, Ghita

Ghobadloo, Shahrokh Mohammadzadeh

Gholami, Fatemeh

Ghomi, Mahmoud

Ghonaim, Hassan

Ghosh, Arnab

Ghosh, Rajeshwary 
Ghosh, Santosh

Ghosh, Somiranjan

Ghosh, Soumita

Ghosh, Subhajit

Ghosh, Sumit

Ghoshal, Kalpana

Giacobini, Ezio G.

Giacomazza, Daniela

Giacomelli, Luca

Giacomello, Emiliana

Giacomini, Elisa

Giacomo, Viviana Di

Giagulli, Cinzia

Giallauria, Francesco

Giamberardino, Maria Adele

Giamogante, Flavia

Giampieri, Enrico

Gianesello, Lisa

Gianfredi, Vincenza

Giangrande, Angela

Gianluca, Carnevale

Giannaccare, Giuseppe

Giannakouros, Thomas

Giannattasio, Michele

Giannetto, Claudia

Giannis, Athanassios

Giannoni, Elisa

Giannoni, Federico

Giannoutsou, Eleni

Gianoncelli, Letizia

Giansanti, Luisa

Gianturco, Luigi

Giardina, Paola

Giarola, Valentino

Gibbs, Bernhard F.

Gibellini, Lara

Gibney, Noel

Gibson, Angela L. F.

Gibson, Susan I.

Giebultowicz, Jadwiga M.

Giełdoń, Artur

Gieras, Anna

Gierszewska, Magdalena

Gigli-Bisceglia, Nora

Giglio, Pierre

Gigliotti, Casimiro Luca

Gijón-Nogueron, Gabriel

Gil Fernández, Vanessa

Gil, Jose Fernando

Gil, Justyna

Gil, Krzysztof
Gil, Minchan

Gil, Robert

Gil, Rosario

Gilbert, Caroline

Gilbo, Nicholas

Giles, Wayne

Giliopoulos, Dimitrios

Gill, Harsharn

Gilli, Francesca

Gilloteaux, Jacques

Gil-Mohapel, Joana M.

Gilot, David

Gils, Janine Van

Gimpel, Matthias

Ginaldi, Lia

Gindraux, Florelle

Ginebra, María Pau

Giner-Tarrida, Luís

Ginsberg, Stephen

Ginzburg, Yelena Z

Giordano, Elena

Giordano, Emanuele

Giordano, Flavio

Giordano, Maria

Giordano, Raffaele

Giordano, Salvatore

Giorgetti, Alejandro

Giorgi, Mauro

Giorgi, Ugo De

Giorgianni, Francesco

Giorgini, Elisabetta

Giovacchini, Giampiero

Giovannelli, Pia

Giovannetti, Elisa

Giovannini, Annalisa

Giovannini, Catia

Giovannoni, Roberto

Giovannucci, David R.

Giovarelli, Matteo

Giovarelli, Mirella

Giovino, Antonio

Giraldo, Pilar

Girao, Henrique

Girault, Alban

Giri, Banabihari

Giri, Hemant

Girolami, Marco

Giron-Gonzalez, Maria D.

Giroud, Maude

Gisbert-Garzarán, Miguel

Gish, Robert 
Gisriel, Christopher

Gisselbrecht, Christian

Giubellino, Alessio

Giudice, Linda C.

Giudice, Valentina

Giudici, Fabiola

Giuffrè, Mauro

Giuffrida, Maria Laura

Giuffrida, Paolo

Giuffrida, Rosario

Giugliani, Roberto

Giuliani, Anna Lisa

Giulianini, Piero Giulio

Giuliano, Michela M.

Giurg, Mirosław

Giuseppina, Basini

Giusti, Ilaria

Gjorgjieva, Monika

Gkaliagkousi, Eugenia

Gkika, Dimitra

Głąbska, Dominika

Gladish, Daniel K.

Gladkikh, Irina

Gladysheva, Inna P.

Gładysz, Anna

Glaser, Bruno

Glass, Donald

Glass, Jon

Glatt, Sebastian

Glavač, Damjan

Glazar, Irena

Glazińska, Paulina

Glazunova, Olga A.

Glazyrin, Yury

Gliszczynska, Anna

Glossmann, Hartmut

Glover, Anthony R.

Głowacka, Katarzyna

Glubb, Dylan

Glubb, Dylan M.

Gluck, Jessica

Glukhov, Alexey

Glukhova, Olga E.

Glushakov, Alexander

Gnanaprakasam, Jaya

Gnanasundram, Sivakumar Vadivel

Gnani, Daniela

Gniadecki, Robert

Gniazdowska, Ewa

Gobbi, Alberto

Goberdhan, Deborah
Goc, Anna

Goda, Katalin

Goda, Keisuke

Goddard, Alan

Godena, Sara

Goderska, Kamila

Godlewska, Marlena

Godlewski, Janusz

Godos, Justyna

Goeser, Felix

Goettig, Peter

Goetzman, Eric S.

Goffinet, Christine

Goggin, Danica E.

Goglia, Fernando

Gojo, Satoshi

Gok, Ozgul

Golay, Josee

Golda, Anna

Goldberg, Erwin

Goldenkova-Pavlova, Irina

Golding, Jon P.

Goldstein, Allan

Goldstein, Andrew S.

Gołembiowska, Krystyna

Golestaneh, Nady

Golgovici, Florentina

Golias, Tereza

Golinelli, Marie-Pierre

Golla, Upendar

Golokhvast, Kirill

Golombick, Terry

Golovleva, Irina

Golubkina, Nadezhda A.

Golubnitschaja, Olga

Golumbeanu, Monica

Gomaraschi, Monica

Gombart, Adrian

Gombert, Alexander

Gomes, Joao R

Gomes, Manuela E.

Gomes, Paula A. C.

Gomes, Pedro

Gomes, Sónia

Gómez Castellà, Cristina

Gomez Del Moral, Manuel

Gómez García, Carlos J.

Gomez Roldan, Maria Victoria

Gomez, Guillermo

Gomez, Nidia

Gómez, Pilar Sánchez 
Gómez-Barrero, Susana

Gomez-Gil, Veronica

Gómez-González, Belén

Gomez-Nino, Angela

Gómez-Pastor, Rocío

Gómez-Puertas, Paulino

Gómez-Sagasti, María T.

Gómez-Zorita, Saioa

Gomoiu, Ioana

Gomzikova, Marina

Gonano, Luis

Gonçalves, Carlos Alberto

Gonçalves, Lídia

Gonçalves, M. Sameiro T.

Gonçalves, Nádia P.

Gonçalves, Renato

Gonçalves, Sónia

Gonda, Sándor

Gondhalekar, Ameya D.

Gondi, Christopher S

Gong, Liangwei

Gong, Siew-ging

Gong, Zifan

GONGAL, Gyanendra

Goni, Felix

Goñi, Fernando R

Gonkowski, Sławomir

Gono, Takahisa

Gonsalves, Wilson I.

Gonzalez Alvarez, Isabel

Gonzalez Mariscal, Isabel

Gonzalez, Antonio

Gonzalez, Deyarina

Gonzalez, Diego Luis

González, Florenci V.

Gonzalez, Gabriel

González, J.F.

González, Jerónimo

González, Marta

Gonzalez, Martin Villalba

Gonzalez, Rogelio

Gonzalez, Victor

Gonzalez-Barcala, Francisco-Javier

Gonzalez-Bulnes, Antonio

Gonzàlez-Duarte, Roser

Gonzalez-Escamilla, Gabriel

González-Fernández, Lauro

González-García, Ismael

González-Iglesias, Héctor

González-Lafont, Àngels

Gonzalez-Menendez, Pedro
González-Minero, Francisco José

Gonzalez-Molina, Jordi

González-Moro, María Begoña

González-Navajas, José M.

González-Navarro, Herminia

González-Nieto, Daniel

González-Sarmiento, Rogelio

González-Sarrías, Antonio

González-Vázquez, Arlyng

Gonzalez-Vicente, Agustin

Goodla, Lavanya

Goodman, Cynthia L.

Goodman, Richard E.

Goodrich, Jaclyn

Goodwin, Christopher

Góra, Artur

Góra, Michalina J.

Goradia, Nishit

Gora-Sochacka, Anna

Gorbacheva, Liubov

Gorbatyuk, Oleg S.

Gordeeva, Olga

Gordon, Darren M.

Gorelik, Julia

Gorgieva, Selestina

Gorgoulis, Vassilis

Gorini, Francesca

Gorio, Alfredo

Goris, Tobias

Gorji, Ali

Gorman, Adrienne

Gornati, Rosalba

Gornowicz, Agnieszka

Gorovits, Boris

Gorrell, Mark

Gorris, Mark

Gorshkova, Ekaterina N.

Gorza, Luisa

Gosav, Evelina Maria

Gosciniak, Grazyna

Gośliński, Tomasz

Gossman, Toni

Goto, Katsumasa

Goto, Kenichi

Goto, Rieko

Goto, Yusuke

Goto-Inoue, Naoko

Götte, Martin

Gottikh, Marina

Götz, Klaus-Peter

Gotzias, Anastasios 
Goua, Marie

Goudarzi, Maryam

Gouguet, Paul

Goukassian, David A.

Gould, Kathleen L.

Gould, Robert W.

Gourdie, Robert

Goureau, Olivier

Gourmans, Marie-José

Gous, Peter

Gout, Ivan

Gouveia, Alexandra M.

Govindan, Prasanth

Govindhan, Ganesan

Gowda, Chandrika

Goyal, Etika

Goyal, Raman

Goyal, Yogesh

Gozzini, Antonella

Grabarek, Beniamin Oskar

Grabarek, Joanna B.

Grabauskas, Gintautas

Grabiec, Urszula

Grabner, Daniel S.

Grabowski, Slawomir

Grabrucker, Andreas M.

Graczyk-Jarzynka, Agnieszka

Grad, Sibylle

Gradia, Daniela

Gradoni, Luigi

Gradov, Oleg V.

Gradova, Margaret

Graether, Steffen

Grafi, Gideon

Gragnano, Felice

Graier, Wolfgang

Gramatica, Paola

Gramignoli, Roberto

Gramolelli, Silvia

Gramsbergen, Jan

Granado, Jose Maria Gonzalez

Granados-Principal, Sergio M.

Grande Tovar, Carlos David

Grande, Raffaele

Grangette, Corinne

Granhag, Lena

Granito, Alessandro

Grant, George

Grant, William

Granzier, Henk L.

Grãos, Mário
Graphodatsky, Alexander

Grasman, Jonathan

Grasshoff, Christian

Grassi, Fabrizio

Grassi, Francesco

Grasso, Giuseppe

Gratchev, Alexei

Grattagliano, Ignazio

Grau, Veronika

Graves, Steven W

Gray, Douglas A.

Gray, Janet M.

Gray, Rachael

Grayson, Dennis R.

Graziano, Giuseppe

Grazul-Bilska, Anna

Grčević, Danka

Grebenkov, Denis S.

Greb-Markiewicz, Beata

Greco, Marta

Greco, Pantaleo

Greco, Simona

Greco, Todd

Greco, Viviana

Green, Erin

Green, Michael

Green, Nicola H.

Greenhalgh, Andrew David

Greenlee, Justin J.

Greenlee-Wacker, Mallary

Greenwood, Michael T.

Gregis, Veronica

Grégoire, Sébastien

Gregorini, Marilena

Gregorio, Gálvez-Valdivieso

Gregório, Michele

Gregory, Christopher D

Gregory, Stephen

Gregory, Steven

Greiner, Jack V.

Greiner, Johannes

Greisen, Per Jr

Greither, Thomas

Grelet, Simon

Grewal, Raji Paul

Grewal, Thomas

Gribsholt, Sigrid Bjerge

Griffis, Eric R.

Griffiths, Cara A.

Griffiths, Rian

Grignani, Giovanni 
Grigorieva, Elvira V.

Grigsby, Christopher L.

Grill, Magdalena

Grillo, Giorgio

Grimaldi, Anna Maria

Grimaldi, Annalisa

Grimaldi, Paola

Grimanelli, Daniel

Grimaudo, Maria Aurora

Grimes, Mark L.

Grimholt, Unni

Grimm, Christina

Grimm, Marcus

Grimm, Marcus O.W.

Grimm, W. D.

Griñán-Ferré, Christian

Grinberg, Daniel

Grippo, Paul J

Gris, Denis

Grisk, Olaf

Gritli-Linde, Amel

Grivas, Petros

Grizzi, Fabio

Grob, Koni

Grobet, Luc

Grodzik, Marta

Groen, Richard

Grogan, Gideon

Groh, Isabel Anna Maria

Grolli, Stefano

Grones, Peter

Groome, James

Grootveld, Martin

Gros, Stephanie J.

Groschner, Klaus

Grose, Julianne

Grošelj, Urh

Gross, Briana L.

Gross, Gideon

Gross, Joshua B.

Gross, Stephane R.

Grossbach, Andrew

Grosse-Brinkhaus, Christine

Grossi, Valentina

Grossman, Ashley Barry

Grosso, Filipa

Groszewicz, Pedro B.

Grothe, Claudia

Grötzinger, Carsten

Groux-Degroote, Sophie

Groveman, Bradley
Grubbs, Nathaniel

Grube, Susanne

Gruber, Ansgar

Grubić Kezele, Tanja

Grubman, Marvin

Grudnik, Przemyslaw

Grul'ová, Daniela

Grünert, Ulrike

Grunewald, Richard

Grunin, Michelle

Grunst, Melissa

Gruntenko, Nataliya

Gruszczynska-Biegala, Joanna

Gruszka, Alicja M

Gruszka, Damian

Gryta, Marek

Grywalska, Ewelina

Grzegrzółka, Jẹdrzej

Grzela, Dawid P

Grzeskowiak, Bartosz

Grzyb, Joanna

$\mathrm{Gu}$, Bin

Gu, Chunju

Gu, Feng

Guadagni, Stefano

Guadagno, Elia

Guagnano, Maria Teresa

Gualerzi, Claudio O.

Gualillo, Oreste

Guallar, Diana

Guan, Qingdong

Guangbi, Li

Guardado-Calvo, Pablo

Guardia, Carlos

Guarnieri, Simone

Guarnieri, Tiziana

Guarraci, Fay

Gubanska, Iga

Gubareva, Elena

Gubin, Denis

Gucciardo, Erika

Gudbergsson, Johann Mar

Gude, Natalie

Gudermann, T

Guéant, Jean Louis

Guemez, Alicia

Guérardel, Yann

Guerin, Coralie

Guerlesquin, Francoise

Guerra, Carmen

Guerra, Flora 
Guerra-Librero, Ana

Guerreiro, Joana F

Guerrero, Carmen

Guerrero-Plata, Antonieta

Guerriero, Giulia

Guerriero, Jennifer

Guerrini, Gabriella

Guest, David

Guevara González, Ramón

Gugliandolo, Agnese

Gugliandolo, Enrico

Gugnoni, Mila

Guha, Prasun

Guiamet, Juan-José

Guibert, Christelle

Guida, Francesca

Guida, Michele

Guiderdoni, Emmanuel

Guidolin, Aline

Guidolin, Diego

Guignard, Gaëtan

Guillamat-Prats, Raquel

Guillén, María Isabel

Guillermet Guibert, Julie

Guillon, Christophe

Guillot, Xavier

Guillou, Herve

Guinovart, Joan J

Guisasola, Eduardo

Guix, Francesc Xavier

Gujas, Bojan

Gulati, Sachin

Güldiken, Nurdan

Gulei, Diana

Gulic, Tamara

Gulin, Alexander A.

Gulino, Ferdinando Antonio

Gulino, Rosario

Gullberg, Donald

Gullbrand, Sarah E.

Guller, Anna

Gullì, Mariolina

Gully, Djamel

Gulyaeva, Natalia V.

Gumienny, Tina

Gumulec, Jaromir

Gunaje, Jayarama

Gunasekar, Susheel K.

Gundamaraju, Rohit

Gundersen, Cameron B.

Günes, Cagatay
Guney, Emre

Gunia-Krzyżak, Agnieszka

Gunn, Alistair J.

Gunning III, William T

Gunning, Peter

Gunn-Moore, Danielle

Guns, Pieter-Jan D.

Günzel, Dorothee

Guo, Bin

Guo, Fenghai

Guo, Qiuquan

Guo, Ting

Guo, Tingting

Guo, Wei

Guo, Xiufang

Guo, Yong

Guo, Zhefeng

Guo, Zhenheng

Guo, Zhongmao

Gupta, Devendra

Gupta, Dipika

Gupta, Gagan D.

Gupta, Mohan

Gupta, Mohit

Gupta, Nilaksh

Gupta, Nishant

Gupta, Parul

Gupta, Prachi

Gupta, Rajat

Gupta, Ramesh C.

Gupta, Rashmi

Gupta, Rishein

Gupta, Sanjay

Gupta, Suprit

Gupta, Sweta

Gupta, Vijayalaxmi G.

Gupta, Yash

Gurgul, Artur

Guriec, Nathalie

Gurrapu, Sreeharsha

Guruli, Georgi

Gurusamy, Raman

Gushchin, Artem L.

Gust, Kurt A.

Gustav, Stalhammar

Gustin, Kurt

Gutberlet, Thomas

Gutiérrez, Laura

Gutiérrez-Adán, Alfonso

Gutierrez-Aguilar, Manuel

Gutierrez-Camino, Angela 
Gutiérrez-Gallego, Ricardo

Gutiérrez-Juárez, Roger

Gutierrez-Merino, Carlos

Gutowska, Izabela

Gutowska-Owsiak, Danuta

Gutsaeva, Diana

Guy, Alexandre

Guyot, Boris

Guzhova, Irina V.

Guzik, Agnieszka

Guzmán Ruiz, Rocío

Guzman, Carlos

Guzman, Fanny

Guzow-Krzemińska, Beata

Gwin, Katja

Gyselaers, Wilfried

Gyselaers, Wilfried J.A.

Gyula, Péter

Ha, Bo-Keun

$\mathrm{Ha}$, Eunyoung

Ha, Jung-Heun

Ha, Ki-Tae

Ha, Yun-Sok

Haak, David

Haanes, Kristian

Haaparanta-Solin, Merja

Haarmann-Stemmann, Thomas

Haas, Oskar

Haase, Hajo

Haastert-Talini, Kirsten

Habas, Khaled

Hack, Ethan

Hackauf, Bernd

Hacker, Ulrich

Hackfort, Bryan T.

Hackl, Hubert

Hada, Masahiko

Hadano, Shinji

Hadjiargyrou, Michael

Hadjikakou, Sotiris K

Ha-Duong, Nguyet-Thanh

Hadwiger, Jeffrey A.

Haendler, Bernard

Haensch, Gertrud Maria

Hafeez, Bilal

Hafizi, Sassan

Häfner, Norman

Hafner-Bratkovič, Iva

Haga, Yutaka

Hage, Ronald

Hagedorn, Claudia
Hagerman, Randi J.

Haghshenas, Sami Shaffiee

Hagidimitriou, Marianna

Hagos, Engda G.

Hagos, Yohannes

Haguet, Vincent

Hahn, Heidi

Hahn, Marc Benjamin

Hahn, Oliver

Hahne, Jens

Haigh, Anthony

Haile, Jemanesh

Hailes, Helen C.

Haines, Jeffery

Haitin, Yoni

Hajdari, Avni

Hajduch, Eric

Hajime, Hirase

Hakamada, Kenichi

Halachmi, Sarel

Halász, Júlia

Halder, Amit Kumar

Halder, Avik

Haley, Nicholas J.

Halitschke, Rayko

Halkia, Evgenia

Hall, Duane

Hall, Rebecca

Hallberg, Einar

Halle, Mari K.

Haller, Steven T.

Hallerman, Eric

Hallett, Maurice B.

Hallmann, Ewelina

Halmos, Balazs

Halmos, Gábor

Hálová, Ivana

Halsall, David J.

Ham, Won-sik

Ham, Young Wan

Hamad, Abdel

Hamada, Masakazu

Hamaguchi, Masahide

Hamamouch, Noureddine

Hamanishi, Junzo

Hamann, Martine

Hamano, Sayuri

Hamano, Tadanori

Hamaoka, Takafumi

Hamar, Peter

Hamazaki, Jun 
Hamel, Patrice P.

Hamel, Rodolphe

Hamidi, Tewfik

Hamieh, Mohamad

Hamilton, Duane H.

Hamilton, Gerhard

Hammer, Elke

Hammer, Quirin

Hammer, Sabine E.

Hammers, David

Hamming, Jaap

Hammond, Billy R.

Hammond, Timothy G.

Hammoudi Halat, Dalal

Hamon, Jean-René

Hampton, Randolph Y.

Han, Changseok

Han, Dohyun

Han, Dong

Han, Dong-Wook

Han, Inbo

Han, Jinming

HAN, Kyu-Man

Han, Liang

Han, Mingyuan

Han, Mira

Han, Pingping

Han, Sang-Wook

Han, Song

Han, Soo Deok

Han, Sun-Young

Han, Xiaobin

Han, Yan

Han, Yanhui

Han, Yuyan

Hanai, Jun-ichi

Hanas, Jay

Hanba, Yuko T.

Hanchate, Naresh

Hancock, Charles Nathan

Hancock, John

Hancu, Gabriel

Handa, Avtar K.

Handa, Osamu

Handle, Florian

Handler, Johannes

Handral, Harish

Handschin, Martin Grossmann

Handzlik, Jadwiga

Hane, Masaya

Hanefeld, Ulf
Haneke, Eckart

Hanemann, Oliver

Haney, Conor M.

Hanganu, Daniela

Hangelbroek, Roland W. J.

Hanisch, Franz-Georg

Hanke, Nina

Hankinson, Oliver

Hankir, Mohammed K

Hanley, Chirstopher

Hannan, Anthony J

Hanne, Hoffmann

Hansel, Christian

Hansen, Keith A.

Hansen, Paul Robert

Hansford, Karl

Hansíková, Hana

Hanson, Kirsten

Hanson, Maureen

Hanson, Nancy D.

Hanss, Michel

Hantusch, Brigitte

Hanus-Fajerska, Ewa Joanna

Hanyuda, Akiko

Hao, Mingang

Hao, Yajing

Haorah, James

Haq, Iram

Haqshenas, Gholamreza

Hara, Akira

Hara, Emilio Satoshi

Harada, Hisashi

Harada, Mamoru

Harada, Masako

Haraguchi, Akira

Haraguchi, Ryuma

Haraguchi, Tokuko

Haragus, Horia

Harald, Claus

Harandi, Vahid M.

Harashima, Nanae

Harazny, Joanna M.

Harbison, Susan T.

Harbuzariu, Adriana

Hardee, Justin P

Hardies, Stephen C.

Hardingham, Jennifer E.

Hardion, Laurent

Hardonnière, Kévin

Harduin-Lepers, Anne

Hardwick, James P. 
Hardwidge, Philip

Hardy, John George

Hardy, Rowan

Hare, Joshua M.

Harezlak, Katarzyna

Harford-Wright, Elizabeth

Hargitai, Rita

Hariharan, Seethalakshmi

Harijan, Rajesh K

Harijith, Anantha

Harkany, Tibor

Harm, Stephan

Harn, Horng-Jyh

Harnor, Suzannah

Haro, Diego

Harold, Denise

Harpaz, Dorin

Harper, Alan

Harrel, Stephen K.

Harrington, Charles

Harrington, Sophie A.

Harris, Carla

Harris, Edward N.

Harris, William S.

Harrison, Paul H. M.

Harrison-Bernard, Lisa

Harrowfield, Jack

Hart, David

Hart, Peter C.

Hartman, Mariusz

Hartman, Matthew C.T.

Hartmann, Matthias

Hartmann, Roland

Hartsfield, James K.

Hartsough, Edward

Hartwig, Thomas

Haruhara, Kotaro

Haruta, Miyoshi

Harvey, Adam

Harvey, Alexandra

Harzer, Klaus

Has, Cristina

Hasan, Raquibul

Hasanuzzaman, Mirza

Hasche, Daniel

Hase, Yoshihiro

Hasegawa, Paul M.

Hasenstein, Karl $\mathrm{H}$

Hashemi, Mohtadin

Hashemolhosseini, Said

Hashim, Adnan
Hashim, Ibrahim A.

Hashimoto, Makoto

Hashimoto, Yoshitaka

Hashizume, Osamu

Haskó, György

Haslwanter, Denise

Haspula, Dhanush

Hassan, Mohamed

Hassell Jr., James E.

Hasterok, Robert

Hastings, Michelle L.

Hastings, Philip J.

Hata, Tamako

Hatano, Etsuro

Hatano, Noriyuki

Hatano, Yuichiro

Hatem, Stephane

Hathaway, Nathaniel

Hattori, Yuichi

Hatzipetros, Theo

Hatzl, Stefan

Hau, Peter

Haugen, Håvard J

Hausauer, Amelia K.

Hauser, Peter

Hausmann, Ludger

Hausner, Georg

Hautz, Theresa

Havlik, Jaroslav

Havunen, Riikka

Hawi, Ziarih

Hawinkels, Lukas

Hawke, Thomas J

Hawrylak-Nowak, Barbara

Hawthorne, Susan

Hayar, Abdullah

Hayasaka, Haruko

Hayashi, Hisashi

Hayashi, Keitaro

Hayashi, Makoto

Hayashi, Mariko Kato

Hayashi, Mikio

Hayashi, Nagao

Hayashi, Ryuji

Hayashi, Shinichiro

Hayashi, Tomohiko

Hayashi, Yujiro

Hayden, Melvin R.Pete

Haydon, Mike

Hayflick, Susan J.

Haynes, Jennifer 
Haynes, Karmella

Hazak, Ora

Hazane-Puch, Florence

Hazubska-Przybył, Teresa

$\mathrm{He}$, Aina

$\mathrm{He}$, Chunbo

He, Hong

He, Hongliang

He, Jia-Qiang

$\mathrm{He}$, Jin-Sheng

He, Junxian

He, Mingyu

He, Shikun

Heakal, Yasser

Healey, Adam

Healey, Gareth

Heath, P.R.

Heberden, Christine

Hecht, Michael $\mathrm{H}$.

Hedden, Peter

Heddleston, John

Hedhammar, My

Heemskerk, Mirjam

Heerklotz, Heiko

Heery, David M.

Heffer, Marija

Hefferon, Kathleen L.

Hegarty, Shane V.

Hegde, Shivanand

Hegedüs, Csaba

Heger, Jacqueline

Hegyi, Péter

Heidari, Behzad

Heidegger, Isabel

Heikema, Astrid

Heilbronner, Urs

Heim, Frederic

Heinbockel, Thomas

Heindel, Jerry

Heinisch, Jürgen

Heinrich, Ralf

Heitkam, Tony

Heitmar, Rebekka

Heitz, Thierry

Hejatko, Jan

Hejnar, Jiří

Hejtmancik, J. Fielding

Helbig, Karla

Heleniak, Zbigniew

Helfrich, Miep

Helge, Jørn
Helland, Åslaug

Heller, Gerwin

Heller, Janosch

Hellewell, Sarah

Helling, Britney A.

Hellinger, Roland

Hellmann, Nadja

Hellstrand, Kristoffer

Helms, Bernd

Helms, Volkhard

Hemachandra, Reddy. P

Hémadi, Miryana

Hemmersbach, Ruth

Hemming, Karl

Hempel, Günter

Henderson, Robert D.

Henderson-Jackson, Evita

Hendgen-Cotta, Ulrike B.

Hendrik, Bargel

Henen, Morkos A.

Heng, Xiao

Henke, Andreas

Hennerman, Julia

Hennigs, Jan K.

Henningsen, Anders

Henrich, Dirk

Henriksson, Marie Arsenian

Henrique, Rui

Henriques, Ana Gabriela

Henriques, Bárbara J.

Henry, Marc

Henry, Ryan A.

Henry, Yves

Hensel, Götz

Henstridge, Christopher

Herath, H. M. Ayala S.

Herath, Venura

Herbst, Andreas

Herczegh, Pál

Herdean, Andrei

Héricourt, François

Hering, Thomas

Hering-Smith, Kathleen

Hermenean, Anca

Hermoso-Orzáez, Manuel Jesús

Hernandez, Agustin

Hernández, Carmen

Hernandez, Jean-François

Hernandez, Joaquim

Hernandez, Pilar

Hernandez, Victor 
Hernandez-Alcoceba, Ruben

Hernandez-Alonso, Pablo

Hernández-Breijo, Borja

Hernández-Cifre, José Ginés

Hernández-Lucas, I.

Hernández-Martínez, Patricia

Hernández-Mesa, Maykel

Hernández-Núñez, Emanuel

Hernández-Ochoa, Erick O.

Hernández-Rodríguez, César

Herndler-Brandstetter, Dietmar

Hernot, Sophie

Herold-Mende, Christel

Herr, Deron Raymond

Herránz, Héctor

Herrera, Blanca

Herrera, Federico

Herrero, Enrique

Herrero, María Jesús

Herrero-Beaumont, Gabriel

Herrmann, Marietta

Hershfinkel, Michal

Herskin, Mette S.

Herskind, Carsten

Hertel, Robert

Hertwig, Stefan

Hertz, Daniel L.

Hervás Pérez, Juan Pablo

Hervé, Técher

Hervouet, Eric

Herzberg, Martin

Hesemann, Peter

Heslegrave, Amanda

Hessheimer, Amelia J.

Hester, Joanna

Hetényi, Csaba

Hetland, Geir

Heuer, Holger

Hewitt, Sylvia C.

Hey, Jeremias

Hianik, Tibor

Hibi, Masahiko

Hiblot, Julien

Hibrand-St.Oyant, Laurence

Hicks, Andrew

Hiebert, Paul

Hieda, Miki

Higashida, Kazuhiko

Higashijima, Shin-ichi

Higgs, David

Higo, Shimpei
Hikima, Jun-ichi

Hildreth, Blake

Hildreth, Eason

Hildreth, Sherry

Hileman, Lena

Hilgemann, Donald William

Hilger, Christiane

Hill, Grant

Hill, Martin

Hilliard, Tyvette S.

Hilliou, Frederique

Hills, Ronald

Hilty, Markus

Himoto, Takashi

Hindra, Hindra

Hinds, Terry D.

Hines, Dustin J.

Hines, Justin K.

Hingorani, Rittu

Hinkula, Jorma

Hinman, Samuel

Hinssen, Horst

Hinzpeter, Alexandre

Hioe, Catarina E.

Hippe, Berit

Hippo, Yoshitaka

Hirabayashi, Tetsuya

Hiraga, Toru

Hirai, Nobumitsu

Hirano, Katsuya

Hirano, Minako

Hirasaka, Katsuya

Hirasawa, Noriyasu

Hirata, Tetsuya

Hirata, Yoko

Hirata-Tsuchiya, Shizu

Hirel, Bertrand

Hiroaki, Hidekazu

Hirohata, Satoshi

Hiroki, Toyoda

Hirono, Keiichi

Hirsch, Pierre

Hirst, Warren

Hiruma, Kei

Hisaoka-Nakashima, Kazue

Hitachi, Keisuke

Hjelmen, Carl E.

Hlaváč, Viktor

Hlozek, Tomas

Hluska, Tomáš

Hnia, Karim 
Hnilicka, Frantisek

Ho, Cheng-Hsun

Ho, Chengying

Ho, Chia-che

Ho, Eric S.

Ho, Han Kiat

Ho, Jar-Yi

Ho, Shih-Ching

Ho, Thanh

Ho, Tsung-Chuan

Ho, Vincent

Hoang, Nam V.

Hoang, Ngoc Ha

Hoashi, Toshihiko

Hochstrasser, Mark

Höckner, Martina

Hodges, Lynette

Hodgkinson, James

Hodgkinson, Tom

Hodny, Zdenek

Hoebeke, Johan

Hoefer, Imo

Hoefsloot, E.H.

Hoell, Jessica I.

Hoffman, Brad G.

Hoffman, Charles S.

Hoffman, Dax Aaron

Hoffman, Nolan

Hoffmann, Klaus H.

Hoffmann, Michele J.

Hogan, Natacha

HogenEsch, Harm

Hohaus, Stefan

Hohenegger, Martin

Hohenester, Simon D.

Hohensinner, Philipp

Hohmann, Miriam S. N.

Hojan, Katarzyna

Hojjat-Farsangi, Mohammad

Hojo, Hironori

Hokugo, Akishige

Holak, Tad A.

Holalu, Srinidhi V

Holaska, James

Hołda, Mateusz Krystian

Holec, Josef

Holecki, Michał

Holendova, Blanka

Holguin, Nilsson

Holien, Jessica

Holland, Heidrun
Hollenbach, Marcus

Holler, Eggehard

Holliday, L. Shannon

Höllig, Anke

Hollomon, Mario G.

Holloway, John

Holm, Jesper Grønlund

Holme, Jørn Andreas

Holmes, Andrew P

Holmes, Neil

Holmøy, Trygve

Holnthoner, Wolfgang

Holsinger, Damain

Hölter, Sabine

Höltke, Carsten

Holynska, Malgorzata

Homa, Joanna

Homblé, Fabrice

Homma, Miwako Kato

Homma, Takujiro

Homma, Tetsuya

Homman-Ludiye, Jihane

Homma-Takeda, Shino

Homocianu, Mihaela

Homolak, Jan

Honda, Kazuho

Honda, Yoshitomo

Hong, Chang Pyo

Hong, Chwan-Yang

Hong, Hyun Sook

Hong, Jeong Hee

Hong, Ji Hyung

Hong, Ka L.

Hong, Su-Hyung

Hong, Xupeng

Hong, Yeong Ho

Hong, Yi-Ren

Honke, Koichi

Honorio-França, Adenilda

Honrao, Chandrashekhar

Honvo, Germain

Hooda, Jagmohan

Hoogendijk, Mark G

Hoogenkamp, Maarten

Hook, Lauren M.

Hook, Vivian

Hooykaas, Paul

Hopp, Russell

Horai, Yoshiro

Hõrak, Rita

Horakova, Jana 
Horchani, Habib

Horel, Ágota

Horgan, Finbarr

Hori, Hiroyuki

Hori, Kiyosumi

Horike, Shin-ichi

Horký, Pavel

Hörmann, Gregor

Horn, Anselm

Hornig, Nadine

Hornung, Bastian

Horobin, Richard W.

Horovistiz, Ana

Horovitz, Amnon

Horowitz, John D.

Horowitz, Mia

Horsburgh, Ann

Horsky, Jiřri

Horstkorte, Rüdiger

Horstmann, Martin A.

Hortells García, Luis

Hortobagyi, Tibor

Hortobágyi, Tibor

Horton, Edward Roy

Horváth, Ádám

Horváti, Kata

Hosaka, Kayoko

Hoshino, Daisuke

Hoshino, Tamotsu

Hosmillo, Myra

Hosório, Hugo

Hosoya, Akihiro

Hossain, Fokhrul

Hossain, Md Jakir

Hosseinkhani, Hossein

Hosseinpour, Sepanta

Hosui, Atsushi

Hosur, Vishnu

Hotta, Yoshihiro

Hou, Chih-Yao

Hou, Jinjun

Hou, Jue

Hou, Shaoping

Houben, Roland

Hough, Kenneth

Houjou, Hirohiko

House, Imran

Houshmand, Mohammad

Houston, Fiona

Howard, Erin W.

Howe, Hwee Siew
Howell, Phil

Howell, Stephen

How-Kit, Alexandre

Hox, Valérie

Hrabal, Richard

Hrdy, Jiri

Hrelia, Patrizia

Hrenar, Tomica

Hribal, Marta

Hrstka, Roman

Hsaio, Michael

Hsia, Shih-Min

Hsia, Victor

Hsiao, I-Lun

Hsiao, Yu-Sheng

Hsieh, Chien-Ming

Hsieh, Ming Kun

Hsieh, Ming-Fa

Hsieh, Ming-Ju

Hsieh, Pei-Ling

Hsieh, Tusty-Juan

Hsieh, Wen-Tsong

Hsu, Bang-gee

Hsu, Chung-Yuan

Hsu, Fang Rong

Hsu, Feiting

Hsu, Fei-Ting

Hsu, Fu-Yin

Hsu, Kuo-Sheng

Hsu, Li-Sung

Hsu, Ming-Hua

Hsu, Ping Chih

Hsu, Ren-Jun

Hsu, Shang-Te Danny

Hsu, Sung-Po

Hsu, Todd

Hsu, Tsung-I

Hsu, Tuan-Ti

Hsu, Yi-Chiung

Hsueh, Yi-Huang

$\mathrm{Hu}$, Changlong

$\mathrm{Hu}$, Chaur-Jong

$\mathrm{Hu}$, Dong Gui

$\mathrm{Hu}$, Guoku

$\mathrm{Hu}$, Guoli

$\mathrm{Hu}$, Jian

$\mathrm{Hu}$, Junhui

$\mathrm{Hu}$, Liang

$\mathrm{Hu}$, Min

$\mathrm{Hu}$, Po-Sheng

$\mathrm{Hu}$, Qiwen 


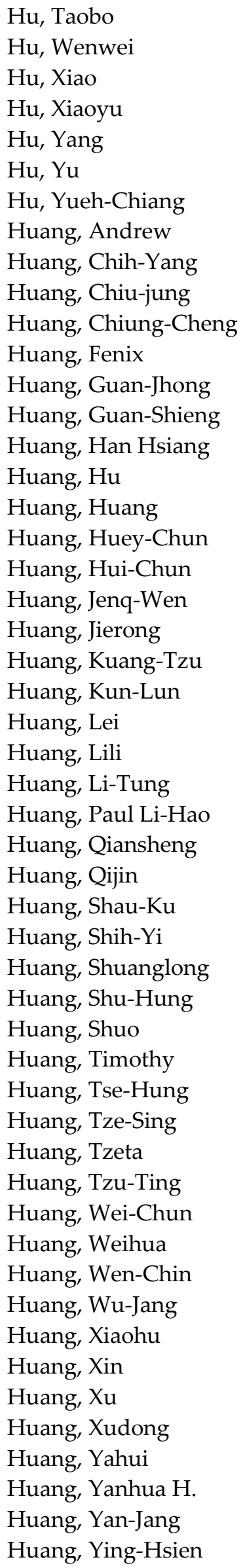

Huang, Yu

Huang, Yu-An

Huang, Yu-Chuen

Huber, Michael

Huber, Rene

Huber, Victor

Hublitz, Philip

Hübner, Max

Huchzermeyer, Bernhard

Huck, Olivier

Huda, Nazmul

Hudlikar, Rasika

Hudson, Andre O.

Hudson, Natalie

Huerta-Angeles, Gloria

Huerta-Yépez, Sara

Hueso, Miguel

Hufbauer, Martin

Hughes, Colan

Hughes, William

Hugo, Eric R.

Hugo, Leon

Hugueney, Philippe

Huh, Jin Hoe

Huleihel, Mahmoud

Hull, J. Joe

Hull, J.Joe

Hulleman, John

Hulme, John

Humayun, Mark S.

Humbert, Magali

Humbert, Patrick O.

Humbolt, Patrice

Hummel, Mary

Hunakova, Luba

Hund, Martin

Hundley, Heather A.

Hung, Andrew

Hung, Kenneth

Hung, Shao-Wen

Hung, Shih-Ya

Hung, Tai-Feng

Hung, Weiting

Hung, Yao-Ching

Hung, Yu-Ju

Hunt, Tamerah

Hunter, Charles T.

Huntington, Nicholas David

Huntosova, Veronika

Hupp, Sabrina

Huppé-Gourgues, Frédéric 
Huppert, Stacey

Huppertz, Berthold

Hur, Yoonkang

Hura, Tomasz

Hurwitz, Stephanie

Husnjak, Koraljka

Hussain, Ahmad Fawzi

Hussain, Mubshar

Hussain, Muhammad Sajid

Hussain, Rumana N.

Hussain, Saman

Hussaini, Haizal

Hussein, Maytham

Hutchins, Andrew P

Hutchinson, John N

Hutmacher, Dietmar

Hüttemann, Maik

Hutter, Michael

Hutterer, Georg C.

Huuskes, Brooke Maree

Huuskonen, Mikko Tuomas

Huvenne, Wouter

Huxford, Tom

Hwa, John

Hwang, Chang-il

Hwang, Changmo

Hwang, Ho Sik

Hwang, In Koo

Hwang, Jae Sung

Hwang, Jeeyeon

Hwang, Justin

Hwang, Pung Pung

Hwang, Wonmuk

Hwang, Yongsung

Hwang-Verslues, Wendy W.

Hwu, Wuh-Liang

Hyatt, Hayden

Hyeonso, Ji

Hyer, Steve

Hyink, Deborah Pinson

Hyun, Young-Min

Hyun-jung, Park

Iaccarino, Guido

Iaccarino, Ingram

Iaccino, Enrico

Iacoangeli, Alfredo

Iacono, Diego

Iadarola, Paolo

Ian, Walsh

Ianchis, Raluca

Iannazzo, Daniela
Iannelli, M. A.

Iannolo, Gioacchin

Iaria, Carmelo

Iasevoli, Felice

Iatsenko, Igor

Ibarra Molero, Beatriz

Iben, Sebastian

Ibrahim, Ahmed

Ibrahim, Mohab M.

Ibrahim, Mohamed Ali

Ibrahim, Sulaiman Sadi

Ichihashi, Masamitsu

Ichikawa, Takahiro

Iconomidou, Vassiliki

Idda, Maria Laura

Ide, Hisamitsu

Idili, Andrea

Ido, Yasuo

Idogawa, Masashi

Ieni, Antonio

Ientile, Riccardo

Ieraci, Alessandro

Ierardi, Enzo

Ifergan, Igal

Igamberdiev, Abir U.

Igartua, Ernesto

Igbaria, Aeid

Igea, Ana

Iglesias Fernández, Raquel

Iglesias, Maria Del Rosario

Iglesias-Linares, Alejandro

Iglesias-Osma, Maria Carmen

Ignatieva, Elena $\mathrm{V}$.

Ignatov, Alexander

Ignatowska-Jankowska, Bogna M.

Ignjatovic, Nenad

Igual, Marta

Ihnatko, Robert

Ihrie, Rebecca

Iida, Kei

Iijima, Kazutoshi

Iizuka, Katsumi

Ikeda, Masahiro

Ikeda, Masato

Ikeda, Tohru

Ikeh, Mélanie

Ikehara, Kenji

Ikeno, Shinya

Ikura, Teikichi

Ilag, Leodevico L.

Ilagan, Ma. Xenia G. 
Ilari, Andrea

Ilchibaeva, Tatiana

Ildefonso, Cristhian J.

Ilia, Gheorghe

Ilic, Nebojsa

Illes, Peter

Illien-Jünger, Svenja

Im, Chang-Nim

Im, Seung-Soon

Im, Sun

Imai, Hiroo

Imai, Jun

Imai, Yoichi

Imamura, Masahiro

Imamura, Sousuke

Imani, Mahdi

Imbeault, Michael

Imbert, Véronique

Imbimbo, Bruno

Imbriano, Carol

Imig, John

Imparato, Giorgia

Imperatore, Concetta

Imran, Qari Muhammad

Inagaki, Shinobu

Inbraj, Stephen

Incarnato, Danny

Inchingolo, Francesco

Incitti, Tania

Incoronato, Mariarosaria

Indini, Alice

Indovina, Paola

Inestrosa, Nibaldo C.

Infante, Arantza

Infante, Marco

Infante, Paola

Ingendoh-Tsakmakidis, Alexandra

Ingham, Aaron B.

Inglada-Pérez, Lucía

Ingram-Smith, Cheryl

Iniesta Cuerda, María

Innchi, Lee

Innocenti, Metello

Inokuchi, Jin-Ichi

Inoue, Haruhiko

Inoue, Haruhisa

Inoue, Keiichi

Inoue, Tatsuya

Inoue, Tsuyoshi

Inoue, Yusuke

Inselman, Amy
Inserte, Javier

Inubushi, Toshihiro

Inuzuka, Hiroyuki

Inyushin, Mikhail Y.

Inzana, Thomas J.

Ioan Voicu, Stefan

Ioannis, Giannakou

Iolascon, Giovanni

Iommelli, Francesca

Ionescu, Daniela C.

Ionita, Gabriela

Ionita, Petre

Iop, Laura

Iordache, Florin

Iorga, Andrea

Iorga, B. I.

Iorio, Egidio

Iorio, Ronald M.

Iosifidis, Michael

Iotti, Stefano

Iourov, Ivan Y.

Iovane, Andre

Iovene, Marina

Ipseiz, Natacha

Iqbal, Omer

Irace, Carlo

Iram, Surtaj

Irato, Paola

Irie, Tetsumi

Irimiciuc, Stefan-Andrei

Irving, Tom

Irzyniec, Tomasz

Isabel Veiga, Maria

Isabey, Daniel

Isacson, Ole

Isakova-Sivak, Irina

Isani, Gloria

Isayama, Hiroyuki

Isemura, Mamoru

ISHIAI, Masamichi

Ishibashi, Kenichi

Ishibashi, Nobuyuki

Ishibashi, Osamu

Ishibazawa, Akihiro

Ishida, Mari

Ishidate, Takao

Ishigami, Tomoaki

Ishihara, Keiichi

Ishii, Hideki

Ishii, Masakazu

Ishii, Tetsuro 
Ishikane, Shin

Ishikawa, Eiichi

Ishikawa, Kiyotake

Ishikawa, Ryuji

Ishimoto, Takahiro

Ishimoto, Takatsugu

Ishitsuka, Yoichi

Ishitsuka, Yosuke

Ishizaki, Azumi

Ishizaki, Takuma

Ishizaki, Yasuki

Ishizuka, Takumi

Isidoro, Ciro

Islam, Jahidul

Islam, Md Mohaimenul

Islam, Md Soriful

Islam, Md Tohidul

Islam, Md. Tabibul

Islam, Mohammad Mazharul

Islam, Mohammad Mirazul

Islam, Salim Timo

Islam, Salman

Islam, Soriful

Ismaiel, Adnan

Ismail Ali, Hamed

Ismail, Mariam N.

Isozaki, Takeo

Itabashi, Etsuko

Itakura, Yoko

Itatani, Yoshiro

Ito, Fumitake

Ito, Keiichi

Ito, Shinsaku

Ito, Takamichi

Ito, Yasuhiro

Ito, Yukako

Itoh, Jun-Ichi

Itoh, Yoshifumi

Itou, Junji

Iuga, Dinu

Iuliano, Rodolfo

Ivančić Šantek, Mirela

Ivanda, Mile

Ivanov, Alexander

Ivanov, Andrei

Ivanov, Milan

Ivanov, Stanimir S.

Ivanov, Yuri

Ivanov, Yuri D.

Ivanova, Anela

Ivanova, Donika G.
Ivanova, Ekaterina

Ivanova, Margarita

Ivanova, Svetlana

Ivics, Zoltán

Ivy, Dunbar

Iwai, Atsushi

Iwaï, Hideo

Iwaizumi, Masakazu G

Iwamoto, Masahiro

Iwasaki, Kenta

Iwasaki, Takashi

Iwata, Junichi

Iwata, Takanori

Iwata, Tomoyuki

Iweala, Onyinye

Iyer, Chitra

Iyer, Deepak Narayanan

Iyer, Janani

Iyer, S.P.

Izaguirre, Gonzalo

Izasa, Hisashi

Izatulina, Alina R.

Izawa, Hironori

Izawa, Kazuhiro P.

Izawa, Takeshi

Izawa, Tetsuya

Izdebska, Magdalena

Izquierdo-Rico, Maria José

Izquierdo-Useros, Nuria

Izumi, Yuichiro

Iżykowska, Katarzyna

Izzi, Valerio

Izzo, Luana

Izzo, Paola

Jaaro-Peled, Hanna

Jabbari, Hosna

Jablonowski, Zbigniew

Jablonska, Ewa

Jacevic, Vesna

Jachetti, Elena

Jackson, Simon

Jacob, Christophe

Jacobs, Elizabeth R

Jacobs, Hugues

Jacobs, Roger

Jacobsen, Monica

Jacobson, Kenneth

Jacobs-Sera, Deborah

Jacquin, Elise

Jadeja, Ravirajsinh

Jadhav, Shyamalagauri 
Jadidi, Amir

Jadiya, Pooja

Jae Wook, Yang

Jaffar, Zeina

Jaffe, Eileen

Jagannatha, Rao

Jaggi, Meena

Jaggupilli, Appalaraju

Jagiello, Karolina

Jagiello-Gruszfeld, Agnieszka

Jagla, Krzysztof

Jagodzinski, Filip

Jagus, Rosemary

Jaguva Vasudevan, Ananda Ayyappan

Jähn, Katharina

Jahn, Olaf

Jahng, Wan Jin

Jahnke, Gizella

Jaimes, Edgar A.

Jain, Era

Jain, Miten

Jain, Vaibhav

Jaiswal, Jyoti K.

Jaitovich, Ariel

Jakaria, Md.

Jakas, Andreja

Jakiel, Grzegorz

Jakiela, Bogdan

Jakimovski, Dejan

Jakobsen, Mogens

Jakopovic, Marko

Jakovlevic, Dragana

Jakubík, Jan

Jakubowska, Monika

Jakubowski, Hieronim

Jalali, Beenu Moza

Jalan-Sakrikar, Nidhi

Jalil, Abbe Maleyki Mhd

Jalkanen, Sirpa

Jambrina, Pablo G.

James, John

James, Nicholas G.

James, Rob S.

James, Victoria

Jamesdaniel, Samson

Jameson, David

Jamin, Nadège

Jamnik, Andrej

Jampilek, Josef

Jan Laskowski, Łukasz

Jana, Bimal
Jana, Sayantan

Janakiraman, Harinarayanan

Janas, Adam

Janas, Dawid

Jancsó, Gábor

Janczar, Szymon

Janczarek, Monika

Jandl, Katharina

Janeczko, Monika

Janeczko, Tomasz

Janek, Tomasz

Jang, Aera

Jang, Byoung Kuk

Jang, Byungki

Jang, Hyonchol

Jang, Jichan

Jang, Jinah

Jang, Seonghoe

Janga, Srikanth Reddy

Jangampalli Adi, Pradeepkiran

Janiak, Agnieszka

Janik, Katrin

Janik, Stefan

Janiuk, HIzabela

Janjetovic, Zorica

Jankovic, Brankica

Jankowicz-Cieslak, Joanna Beata

Janousek, Bohuslav

Jans, Judith J.M.

Jansone, Baiba

Jansova, Denisa

Jantzie, Lauren L.

Januchowski, Radosław

Janusz, Grzegorz

Januszko, Adam

Jao, Tzu-Ming

Jaquet, Kornelia

Jaradat, Nidal

Jaramillo-Flores, Maria

Jara-Palomares, Luis

Jarcuska, Peter

Jardin, Isaac

Jarocka-Cyrta, Elzbieta

Jarome, Timothy

Jarończyk, Małgorzata

Jaroszewicz, Jerzy

Jaroszuk - Ściseł, Jolanta

Jarret, Robert L.

Jarstfer, Michael Bruce

Jarugula, Sridhar

Järvinen, Tero A. H. 
Jarzebski, Maciej

Jasińska-Stroschein, Magdalena

Jasoliya, Mittal

Jastrzebska, Beata

Jászai, József

Jat, Parmjit S.

Jaumot, Joaquim

Javadov, Sabzali

Javelle, Emilie

Jawhar, Mohamad

Jaworek, Andrzej Kazimierz

Jaworska, Dagmara

Jay, Steven

Jayant, Rahul

Jayanthi, Sankar

Jayaraman, Arul

Jayawardena, Dulari

Jazirehi, Ali R.

JB Van Den Hoff, Maurice

Jean, Christine

Jean, Ludovic

Jean-François, Frantz

Jeanne, Mathieu

Jean-Quartier, Claire

Jedlovszky-Hajdu, Angela

Jedryczka, Malgorzata

Jee, Hyeon-Gun

Jee, Shiouhwa

Jeffree, Rosalind Lindy

Jekabsone, Aiste

Jelokhani-Niaraki, Masoud

Jeltsch, Albert

Jembrek, Maja Jazvinšćak

Jena, Prasant Kumar

Jendelová, Pavla

Jenei-Lanzl, Zsuzsa

Jeng, Chung-Jiuan

Jeng, Jiiang-Huei

Jenkins, Brendan J.

Jenkins, Jill A.

Jenkins, Samir V.

Jensen, Benjamin

Jensen, Eric D.

Jensen, Just

Jensen, Kimmo

Jensen, Lars Henrik

Jensen, Rebekka Vibjerg

Jensen-Jarolim, Erika

Jeon, Sohee

Jeong, Byung-Hoon

Jeong, Dong-Hoon
Jeong, Dongtak

Jeong, Gil-Saeng

Jeong, Ho-Chang

Jeong, Hyun Young

Jeong, Keun-Yeong

Jeong, Rae-Dong

Jeong, Sekyoo

Jeong, Seon-Yong

Jeong, Seri

Jerca, Florica Adriana

Jerebtsova, Marina

Jeremic, Aleksandar M.

Jermakowicz, Edyta

Jerman, Igor

Jernberg-Wiklund, Helena

Jeroen T, Buijs

Jeronimo, Carmen

Jerzak, Malgorzata Maria

Jerzemowska, Grażyna

Jeseta, Michal

Jesionek, Marcin

Jetten, Anton

Jeung, Eui-Bae

Jewell, Jenna

Ježek, Petr

Jeżewska-Frąckowiak, Joanna

Jha, Mithilesh

Jha, Ramesh K.

Jhanwar, Shalu

Ji, Hong

Ji, Peng

Jia, Jun

Jia, Shang

Jia, William

Jia, Zhe

Jiang, Cheng

Jiang, Chengmin

Jiang, Jean X.

Jiang, Jianxiong

Jiang, Qingfei

Jiang, Shu-Ye

Jiang, Wei

Jiang, Yong

Jiang, Yun

Jiang, Zhenqi

Jiang, Ziwen

Jiantai Timothy, Qiu

Jibran, Rubina

Jicsinszky, László

Jikuzono, Tomoo

Jiménez-Barbero, Jesus 
Jiménez-González, Gema

Jiménez-Jiménez, Félix Javier

Jiménez-Sánchez, María Carmen

Jimi, Eijiro

Jin Boo, Jeong

Jin, Dong-Hoon

Jin, Hongping

Jin, Hye Jin

Jin, Jun-O

Jin, Li

Jin, Tao

Jin, Tengchuan

Jin, Xin

Jin, Yuefei

Jin, Zhaohui

Jindo, Keiji

Jing, Ran

Jing, Wenwen

Jinno, Yohei

Jinrui, Yang

Jinwal, Umesh K.

Jiráček, Jiří

Jirkovská, Anna

Jo, Byung Hoon

Jo, Yong Hun

Joachim, Stephanie

Joachimiak, Ewa

João, Silva

Jobin, Marie-Lise

Jobling, Malcolm

Jobling, Michael

Jócsák, Ildikó

Joe, Eunhye

Johann, Sonja

Johannesen, Katrine M.

Johansson, Anders

Johansson, Pegah

John Jervis, Peter

John, Łukasz

Johnen, Georg

Johner, Albert

Johnsen, Kasper Bendix

Johnson, Boris Gabriel

Johnson, Caroline

Johnson, Colin P.

Johnson, Daniel

Johnson, David G.

Johnson, Erik

Johnson, Gregory

Johnson, Lance A

Johnson, Nicholas
Johnson, Philip

Johnson, Rajasingh

Johnson, Reed F.

Johnstone, Daniel

Johnstone, Elizabeth

Joles, Jaap A.

Jolkkonen, Jukka

Jolly, Mohit Kumar

Jones, Chris

Jones, Corinne A.

Jones, Elena

Jones, Jeffrey A

Jones, John

Jones, Matt

Jones, Meriel

Jones, O.D.

Jones, Peter

Jong, Chianju

Joo, Min Wook

Joo, Yuyoung

Jordan, Chloe

Jordan, Cynthia L.

Jordan, Hannah

Jordan, Peter

Jordan, V. Craig

Jorgačevski, Jernej

Jorge, Feito

Jorge, J. C. Luis

Jorgensen, Andrea

Jorgensen, Jens Otto Lunde

Jörns, Anne

Jorrin-Novo, Jesus V

Josef, Kapfhammer

Joseph, James

Joseph, Justin V

Joshi, Aditya

Joshi, Bharat H.

Joshi, Kamal

Joshi, Pushpa Raj

Joshi-Navare, Kasturi

Jost, Daniel

Jost, Matthias

Josue, Delgado

Joubert, Annie

Joubert, Lydia-Marie

Joyce, Michael A.

Joyce, Susan A.

Jóźwicki, Wojciech

Jozwik, Maciej

Ju, Sang-Yhun

Juan, Chi-chang 
Juan, Hsueh-Fen

Juengel, Jennifer L.

Jugessur, Aju

Juhász, Béla

Juhász, Réka

Juhász, Tamás

Julián, Esther

Julien, Christian M.

Julio, Bastos Arrieta

Jullian, Nathalie

Juluri, Abhishek

Julve, Josep

Jumbo-Lucioni, Patricia

Jun, Chang-Duk

Jun, Sawada

Jung, Alain C.

Jung, Chan Kwon

Jung, Chol-Hee

Jung, Ho Won

Jung, Hyungil

Jung, Inuk

Jung, Jae Hwan

Jung, Jinsei

Jung, Ki-Hong

Jung, Klaus

Jung, Kwang-Mook

Jung, Sascha

Jung, Se Yong

Jung, Sung Chul

Jung, Woo-Jin

Jung, Yuh-Seog

Jungblut, Peter R.

Junger, Wolfgang

Junka, Adam Feliks

Jurasz, Paul K.

Jurczyk, B.

Jurenas, Dukas

Jurič, Damijana Mojca

Jurk, Kerstin

Jurkowska, Halina

Jurkowska, Renata Zofia

Jurynec, Michael J.

Just, Steffen

Juzoń, Katarzyna

Jyotsna, Mishra

Kabakov, Alexander E.

Kabała-Dzik, Agata

Kabir, Morvarid

Kaburagi, Tomoko

Kaca, Wiesław

Kačániová, Miroslava
Kachamakova-Trojanowska, Neli

Kachroo, Priyadarshini

Kącka-Zych, Agnieszka

Kaczmarek, Beata

Kaczmarek, Krzysztof

Kaczmarek-Ryś, Marta

Kaczor, Agnieszka A.

Kaczorek, Ewa

Kaczorowski, Catherine C.

Kaczyńska, Katarzyna

Kadam, Leena

Kadamoto, Suguro

Kaden, Rene

Kaesler, Susanne

Kaether, Christoph

Kafarski, Pawel

Kafel, Alina

Kagami, Masayo

Kagey, Jacob D

Kageyama, Daisuke

Kageyama, Hakuto

Kahaleh, Bashar

Kähäri, Veli-Matti

Kai, Keita

Kai, Mihoko

Kaida, Daisuke

Kailu, Yang

Kain, Vasundhara

Kainulainen, Veera

Kaiser, Thomas M.

Kaitsuka, Taku

Kaja, Simon

Kaji, Hiroshi

Kaji, Kosuke

Kaji, Toshiyuki

Kajiura, Hiroyuki

Kajiya, Hiroshi

Kajiyama, Hiroshi

Kakehashi, Anna

Kakeji, Yoshihiro

Kakinen, Aleksandr

Kakinuma, Sei

Kakinuma, Yoshihiko

Kakizaki, Tomohiro

Kakkar, Ashok

Kakkassery, Vinodh

Kalafateli, Aimilia Lydia

Kalani, Komal

Kalaska, Bartlomiej

Kalathiya, Umesh

Kalatzis, Vasiliki 
Kalayda, Ganna

Kaldis, Philipp

Kale, Abhijit

Kalemba, Ewa M.

Kalen, Amanda

Kalíková, Kvĕta

Kalinec, Federico

Kalinin, Vladimir I.

Kalinina, Olga

Kalinka, Ewa

Kalisz, Andrzej

Kalisz, Stanisław

Kalita-de Croft, Priyakshi

Kallenborn-Gerhardt, Wiebke

Kaller, Markus

Kalló, Gergő

Kallunki, Tuula

Kalluru, Poliraju

Kalogirou, Andreas S.

Kalous, Jaroslav

Kalpachidou, Theodora

Kalra, Divya

Kalska-Szostko, Beata

Kaltsatou, Antonia

Kaltschmidt, Barbara

Kalucka, Joanna

Kamada, Yoshihiro

Kamal, Fadia Ali

Kamal, Muhammad Shahzad

Kamanová, Jana

Kamath, Divya

Kamath, Sandip

Kambe, Taiho

Kamei, Kaeko

Kamenarska, Zornitsa Gancheva

Kamenski, Piotr

Kameshima, Satoshi

Kameya, Masafumi

Kaminaka, Hironori

Kaminska, Bozena

Kamińska, Joanna

Kamiński, Tomasz W.

Kamitori, Shigehiro

Kamiya, Takehiro

Kämmerer, Peer W

Kamnev, Alexander

Kamoto, Toshiyuki

Kamoun, Malek

Kamysz, Elżbieta

Kamysz, Wojciech

Kanada, Masamitsu
Kanakis, Ioannis

Kanamarlapudi, Venkateswarlu

Kanamori, Takao

Kanaoka, Masahiro

Kanata, Eirini

Kanazawa, Masato

Kanczler, Janos M.

Kanda, Atsuhiro

Kanda, Naoko

Kandhasamy, Sathiyaraj

Kandil, Sahar

Kandpal, Manoj

Kaneda, Masahiro

Kanekiyo, Takahisa

Kanelis, Voula

Kanemura, Nobuhiro

Kanetis, Loukas

Kang, CongBao

Kang, David

Kang, Dongchul

Kang, Ellen $\mathrm{H}$.

Kang, Eunju

Kang, Heemin

Kang, Hee-Taik

Kang, Hong-Yo

Kang, Hunseung

Kang, Jae Seung

Kang, Jeong-Hyun

Kang, Jianming

Kang, Jonghoon

Kang, Ju-Hee

Kang, Kwon-Kyoo

Kang, Kyung Pyo

Kang, Li

Kang, Minjee

Kang, Nam Sook

Kang, Sang Sun

Kang, Seungwoo

Kang, Sun-Woong

Kang, Tae-Bong

Kang, Tae-Hong

Kang, Taek Jin

Kang, Wei

Kang, Yang Jae

Kang, Young-Hee

Kangas, Heli

Kani, Kian

Kania, Stephen A.

Kannan, Mathur S.

Kannappan, Ramaswamy

Kanno, Takahiro 
Kano, Hideaki

Kanoi, Bernard

Kant, Shiva

Kanto, Takeshi

Kanugula, Anantha Koteswararao

Kanwar, Yashpal S.

Kanzaki, Hiroyuki

Kao, Li-Ting

Kao, Peter

Kao, Shung-Te

Kapagianni, Pantelitsa

Kapcia, Konrad Jerzy

Kaplan, Barbara

Kaplan, Joshua M.

Kapłon-Cieślicka, Agnieszka

Kappen, Claudia

Kapranov, Sergey

Kapravelou, Garyfallia

Kapsogeorgou, E.K.

Kapur, Neeraj

Kar, Adwitiya

Kar, Anirban

Karabina, Sonia-Athina

Karakaidos, Panagiotis

Karaki, Shin-ichiro

Karaman, Sinem

Karamouzis, Michalis

Karan, Dev

Karantanos, Theodoros

Karapanayiotides, Theodore

Karasawa, Ken

Karasik, David

Karazniewicz, Marta

Karbownik, Agnieszka

Karcagi, Veronika

Karch, Jason

Kardassis, Dimitris

Kardos, Julianna

Kargl, Julia

Karihtala, Peeter

Karimi, Khalil

Karisola, Piia

Kariya, Yoshinobu

Kariyawasam, Harsha

Karkanis, Anestis

Karki, Hari Sharan

Karkowska-Kuleta, Justyna

Karlsson, Erik

Karlsson, Jan-Olof

Karoly, Hollis

Karras, Panagiotis
Karsak, Meliha

Kartsonaki, Christiana

Karuppiah, Vijaykumar

Karvinen, Sira

Karwowski, Boleslaw

Kasai, Fumio

Kaschina, Elena

Kashaninejad, Navid

Kashapov, Ruslan R.

Kashfi, Khosrow

Kashimada, Kenichi

Kashina, Anna

Kashiwagi, Akiko

Kashiwakura, Ikuo

Kashuba, Elena

Kaski, Juan Pablo

Kasparov, Sergey

Kasprzak, Aldona

Kasprzak, Artur

Kasprzak, Magdalena Paulina

Kass, Laura

Kassai, Hidetoshi

Kassassir, Hassan

Kassayová, Monika

Kässmeyer, Sabine

Kasture, Ameya

Kasuga, Kensaku

Kasztan, Malgorzata

Katagiri, Hiroshi

Katagiri, Takenobu

Kataoka, Hiroaki

Kataoka, Tatsuki R

Kataoka, Yosky

Katarzyna, Malarz

Katase, Naoki

Katayama, Shigeru

Katayama, Takeshi

Kate, Mahesh

Kathare, Praveen Kumar

Kathawala, Rishil

Katikou, Panagiota

Katila, Nikita

Katneni, Upendra

Kato, Keita

Kato, Takamitsu A

Kato, Yasumasa

Kato, Yoshio

Kato, Yusuke

Katoh, Kazuo

Katona, Eva

Katona, Gábor 
Katrinli, Seyma

Katsuhara, Maki

Kattner, Lars

Katyal, Priya

Katz, Ben Z.

Kauffman, Kathryn

Kaufmann, Christopher N.

Kaul, Rashmi

Kauppinen, Anu

Kaur, Amanpreet

Kaur, Gurvinder

Kaur, Harleen

Kaur, Simranjeet

Kaushik, Ajeet

Kaushik, Ashlesha

Kaushik, Nagendra Kumar

Kaushik, Neha

Kaushik, Prashant

Kaushik, Shelly

Kaushik, Shivani

Kavanagh, Dean

Kavecan, Ivana

Kawabata, Kyuichi

Kawabori, Masahito

Kawaguchi, Nanako

Kawahara, Masahiro

Kawakami, Hisato

Kawakami, Masanori

Kawakami, Susumu

Kawakatsu, Taiji

Kawakita, Tetsuya

Kawamura, Izuru

Kawamura, Teruhisa

Kawano, Kouichiro

Kawano, Satoshi

Kawasaki, Hiroshi

Kawata, Kazumi

Kawauchi, Keiko

Kawiak, Anna

Kayser, Klaus

Kayser, Reilly R.

Kazakov, Sergey

Kazakova, Oxana B.

Kazama, Tomohiko

Kazeto, Yukinori

Kazlauskas, Arunas

Kazuaki, Taguchi

Kazunori, Matsuda

Ke, Po-Yuan

Keane, Robert W

Kearsey, Stephen
Keating, Elisa

Kędzierska, Ewa

Keeling, Kim

Keereetaweep, Jantana

Kehrel, Beate

Keightley, Cristina

Keiper, Brett D.

Keita, Hady

Kejík, Zdeněk

Kellenberger, Stephan

Keller, Adrian

Keller, Johannes

Keller, Lance

Keller, Nancy P.

Kellermayer, Miklos

Kelley, Dior R.

Kello, Martin

Kelly, Robert

Kemler, Martin

Kemp, Michael G.

Kempisty, Bartosz

Kendall, Tim

Kendal-Wright, Claire E.

Kengelbach-Weigand, Annika

Kenkel, W. M.

Kenmochi, Takashi

Kennedy, Lindsey

Kennedy, Michael A.

Kenney, Kimbra

Kennon-McGill, Stefanie

Kenny, Hilary A.

Kenny, Nathan James

Keogh, Kate

Kepinska, Marta

Kepp, Oliver

Kerckhove, Nicolas

Kern, Ramona

Kerr, Stephanie C.

Kersten, Sander

Kesari, Kavindra Kumar

Kesharwani, Siddharth

Kessel, David

Kesterson, Joshua P.

Keszler, Agnes

Key, Jaehong

Keyel, Peter A.

Keyzers, Robert

Khacho, Mireille

Khadka, Vedbar Singh

Khalaf, Fatimah

Khalf, Abdurizzagh 
Khalighi, Sanaz

Khalyfa, Abdelnaby

Khambu, Bilon

Khammanivong, Ali

Khan, Amjad

Khan, Husain Yar

Khan, Irfan

Khan, Mahmood

Khan, Meraj Alam

Khan, Mohammad Aslam

Khan, Mohd Mohsin

Khan, Mohsin

Khan, Nabab

Khan, Sabbir

Khan, Sajid

Khan, Sanaullah

Khan, Shafiq

Khan, Shanjida

Khan, Sonja

Khan, Wahab A.

Khanal, Sameer

Khananshvili, Daniel

Khanh, Tran Dang

Khanna, Hemant

Khanna, Rajesh

Kharaeva, Zaira F.

Kharche, Sanjay R.

Khattab, Muhammad

Khaw, Kooi Yeong

Khella, Heba

Kheradpezhouh, Ehsan

Khisamutdinov, Emil

Khivansara, Vishal

Khobta, Andriy

Khodanovich, Marina

Khong, Anthony

Khoo, Bee Luan

Khoo, Cheang

Khoshmanesh, Khashayar

Khozin-Goldberg, Inna

Khrustaleva, Liudmila

Khuchua, Zaza

Khung, Yit Lung

Khurana, Namrata

Khurshid, Zohaib

Ki, Sung Hwan

Kiang, Tony K. L.

Kibenge, Frederick

Kichik, Nessim

Kiczorowska, Bożena

Kida, Satoshi
Kido, Mizuho A.

Kieć-Kononowicz, Katarzyna

Kieda, Claudine

Kiefer, Christiane

Kieffer, Bruno

Kieliszek, Marek

Kieran, Troy J

Kietzmann, Thomas

Kiezun, Marta

Kijewska, Monika

Kikis, Elise A.

Kikuyama, Masataka

Kil, Eui-Joon

Kilár, Anikó

Kilari, Sreenivasulu

Kilb, Werner

Kilinc, Devrim

Kilk, Kalle

Kim, A-Ru

Kim, Bongju

Kim, Boo-Young

Kim, Byung S.

Kim, Byung Soo

Kim, Chang-Bae

Kim, Cheorl-Ho

Kim, Choon Young

Kim, Dokyoung

Kim, Dong Hyun

Kim, Dong Seon

Kim, Donggeon

Kim, Dong-Hwan

Kim, Dong-Hwee

Kim, Dong-Kyu

Kim, Donguk

Kim, Ella

Kim, Eunhee

Kim, Haeng-hoon

Kim, Hae-Won

Kim, Hahn Young

Kim, Ha-Neui

Kim, Han-Joon

Kim, Hee-Joon

Kim, Hee-Taek

Kim, Hong Nam

Kim, Ho-Shik

Kim, Hye One

Kim, Hye Ryoun

Kim, Hyeun Sung

Kim, Hyun Ah

Kim, Hyun Joon

Kim, Hyungsik 
Kim, Hyunho

Kim, Hyun-Jung

Kim, Hyun-Soon

Kim, Il-man

Kim, In Jung

Kim, InKyeom

Kim, Jae Bum

Kim, Jae Geun

Kim, Jae Kwang

Kim, Jae Young

Kim, Jaehan

Kim, Jae-Hwan

Kim, Jae-Seok

Kim, Ja-Eun

Kim, Jae-woo

Kim, Jaeyoon

Kim, Jae-Young

Kim, Jee Hwan

Kim, Jee Taek

Kim, Jeong-Hwan

Kim, Jeong-Il

Kim, Jeongtae

Kim, Jin Su

Kim, Jinho

Kim, Jin-Hong

Kim, Jin-Kyung

Kim, Jin-Wook

Kim, Jong-Eun

Kim, Jongkee

Kim, Jongmin

Kim, Joungmok

Kim, Jung Sun

Kim, Jungeun

Kim, Junghyun

Kim, Junmo

Kim, Keun Il

Kim, Ki Hyun

Kim, Ki-Taek

Kim, Kyeongiin

Kim, Kyoung Soo

Kim, Kyounghyun

Kim, Kyoungtae

Kim, Kyung Bo

Kim, Kyung Eun

Kim, Kyunggon

Kim, Kyung-Min

Kim, Kyuseok

Kim, Lenise Jihe

Kim, Mee Kum

Kim, Mi-hyun

Kim, Min Jung
Kim, Min-Hyun

Kim, Minsik

Kim, Min-Soo

Kim, Nam Deuk

Kim, Namkwon

Kim, Nayoung

Kim, Ok-Kyung

Kim, Pyung-Hwan

Kim, Raymond $\mathrm{H}$

Kim, Ryong Nam

Kim, Sang Wan

Kim, Sang-We

Kim, Seil

Kim, Seog Ju

Kim, Seokhee

Kim, Seok-Jun

Kim, Seongjae

Kim, Seyun

Kim, Soochong

Kim, Soo-Hyun

Kim, Sooyeon

Kim, Soo-Youl

Kim, Su Young

Kim, Su-Jeong

Kim, Sun Kwang

Kim, Sun Tae

KIM, Sung Joon

Kim, Sungeun

Kim, Sung-Kun (Sean)

Kim, Tae Il

Kim, Tae Min

Kim, Tae-Hyung

Kim, Tae-Jung

Kim, Tae-Sung

Kim, Won Ho

Kim, Wootae

Kim, Yangmee

Kim, Yong Jin

Kim, Yong $\mathrm{Ku}$

Kim, Yong-Ick

Kim, Yong-Sik

Kim, Yoon Young

Kim, Yoon-Chul

Kim, Young Jun

Kim, Young-Sang

Kimata, Jason

Kimball, Jennifer

Kimber-Trojnar, Żaneta

Kimbrel, Jeffrey

Kimeklis, Anastasiia K.

Kimoto, Michiko 
Kimura, Kazuhiro

Kimura, Seisuke

Kimura, Tomoki

Kimura, Tsuyoshi

Kinarivala, Nihar

Kindermann, Harald

King, Chih-Yen

King, Thomas F.J.

Kino, Tabito

Kinoshita, Chisato

Kinoshita, Takayoshi

Kinthada, Lakshmanakumar

Kioussi, Chrissa

Kirberger, Michael

Kirby, Elizabeth D.

Kirchmair, Johannes

Kirchner, Sebastian

Kireev, Igor I.

Kirimura, Kohtaro

Kirkby, Kenneth

Kirov, Roumen

Kirsanov, Kirill

Kirsch, Roy

Kirschnek, Susanne

Kirschner, Michaela B.

Kirubakaran, Palani

Kisand, K.

Kiselev, Anton

Kiselev, Konstantin V.

Kishigami, Satoshi

Kishimoto, Jiro

Kishimoto, Shinji

Kishimoto, Yo

Kishore Sakharkar, Meena

Kiskova, Terezia

Kiss, Anna L.

Kiss, Attila

Kiss, Csongor

Kiss, Erzsébet

Kiss, Éva

Kiss, Rita

Kiss, Robert

Kitaguchi, Tetsuya

Kitahara, Ryo

Kitajiri, Shin-ichiro

Kitamura, Akira

Kitano, Yoshikazu

Kitaoka, Motomitsu

Kitase, Yukiko

Kitaura, Jiro

Kitazawa, Sohei
Kitsiouli, EIRINI

Kittel, Agnes

Kittl, Sonja

Kiya, Taketoshi

Kiyan, Yulia

Kjaer, Lasse

Kjeldsen, Eigil

Klaiman, Cheryl

Klampatsa, Astero

Klampfer, Lidija

Klapper, Paul

Klar, Agnes

Klassen, Roland

Klatte-Schulz, Franka

Klavins, Linards

Klebert, Szilvia

Kleczkowska, Patrycja

Kleeff, Jörg

Kleibl, Zdeněk

Klein, Britta

Klein, David C.

Klein, Eric

Klein, Gordon L.

Klein, Gracjana

Klein, Janet D

Klein, Kerstin

Klein, Mark A.

Klein-Szanto, Andrés

Klek, Stanislav

Klementieva, Oxana

Klemme, Sonja

Klener, Pavel

Klenov, Mikhail S.

Klepikova, Anna V.

Klimas, Jan

Klimaszewski, Lars

Klimczak, Aleksandra

Klimek, Katarzyna

Klimek-Chodacka, Magdalena

Klimek-Ochab, Magdalena

Klimontov, Vadim

Klink, Magdalena

Klintsova, Anna

Klitgaard, Janne

Klobuch, Sebastian

Kloc, Malgorzata

Klocko, Amy L.

Klontzas, Michail

Klotz, James L

Kluczyk, Alicja

Klupp, Barbara 
Klussmann, Enno

Klyczek, Karen

Kmiec, Eric B.

Knaap, Esther

Knap, Narcyz

Knappskog, Per M.

Knäuper, Vera

Knecht, Hans

Knez, Jure

Knier, Benjamin

Knight, Marc

Knight, Walter E.

Kniss, Douglas A.

Knobloch, Karsten

Knoch, Eva

Knölker, Hans-Joachim

Knoll, Marko

Knoop, Kathryn

Knowles, Helen

Knüchel, Ruth

Knudsen, Jonas R

Ko, CheMyong

Ko, Frank

Ko, Sung-kyun

Ko, Swee-Suak

Kobayashi, Hiroshi

Kobayashi, Jun

Kobayashi, Kazuya

Kobayashi, Koichi

Kobayashi, Masaki

Kobayashi, Satoru

Kobayashi, Tatsuya

Kobayashi, Yayoi

Kobayashi, Yoshiro

Kobold, Sebastian

Kobrak, Mark N.

Kocábek, Tomáš

Koce, Jasna Dolenc

Koch, Clarissa M.

Koch, Kristine

Koch, Wojciech

Kochan, Grazyna T.

Kochmanski, Joseph John

Kochneva, Galina

Kočí, Zuzana

Kocira, Anna

Kocsis, Bela

Kocsy, Gábor

Koczyk, Grzegorz

Kodali, Maheedhar

Kodama, Takahiro
Kodet, Ondrej

Kodidela, Sunitha

Kodja, Hippolyte

Kodziszewska, Katarzyna

Koeffel, Rene

Koenen, Rory

Koga, Hiroyuki

Koga, Hisashi

Koga, Jun-ichiro

Koga, Yoshikatsu

Kogawa, Takahiro

Koh, David

Koh, Gar Yee

Kohanbash, Gary

Kohara, Yukihiro

Köhl, Gudrun

Köhn, Marcel

Kohri, Michinari

Kohwi-Shigematsu, Terumi

Koide, Tetsuya

Koike, Atsushi

Koike, Haruki

Koike, Kenzo

Koirala, Ranjan

Koivisto, Ari

Koivumäki, Jussi

Kojima, Masami

Kojima, Soichi

Kok, Wouter

Kokabu, Shoichiro

Kokh, Daria B

Kokkinidis, Michael

Kokkola, Tarja

Kokotou, Maroula G.

Koksharova, Olga A.

Kolanowski, Jacek L.

Kolar, Mitja

Kolasa-Wołosiuk, Agnieszka

Kolawole, Abimbola

Kolcsár, Melinda

Koldehoff, Michael

Kolesnikova, Tatyana

Koli, Swanand

Kolluru, Gopi

Kolmar, Harald

Kolobov, Alexandr Alexandrovich

Kolodziejski, Pawel Antoni

Kolonko, Aureliusz

Kolosnjaj-Tabi, Jelena

Kolosova, Nataliya

Kolukisaoglu, Üner 
Komada, Munekazu

Komaki, Hisayuki

Komarova, Natalia V.

Komasa, Satoshi

Komati, Rajesh

Komatsu, Hidetoshi

Komatsu, Masanobu

Komatsu, Satoshi

Komatsu, Toru

Komives, Tamas

Kommireddy, Vasu

Komori, Toshihisa

Komuraiah, Myakala

Kon, Shunsuke

Kondo, Hiromu

Kondo, Motonari

Kondo, Tatsuya

Kondoh, Hiroshi

Konduri, Vanaja

Kondylis, Evangelis

Kones, Richard

Konev, Alexander

Kong Thoo Lin, Paul

Kong, Il-Keun

Kong, Kien Voon

Kong, Lisheng

Kong, Qingzhong

Konger, Raymond L.

König, Gerhard

Konishi, Hiroaki

Kono, Michihiro

Kononikhin, Alexey S.

Konopacka-Łyskawa, Donata

Konopelski, Piotr

Konopka, Anna

Konrad, Heino

Konstantakou, Eumorphia G.

Konstantinidis, Theocharis G.

Konstantinov, Spiro M.

Kontek, Bogdan

Kontogianni, Georgia

Kontos, Christos K.

Kontsevaya, Irina

Konturek, Peter Christopher

Koo, Bon-Nyeo

Koo, Hee-beom

Koos, Björn

Koot, Bart G.P.

Kopaczyńska, Marta

Kopanja, Dragana

Kopecký, David
Kopecký, Jan

Kopel, Pavel

Kopjar, Nevenka

Koppe, Janna G.

Kopra, Kari

Koprowski, Piotr

Kopsky, David J.

Kopustinskiene, Dalia M.

Kopyta, Ilona

Korac, Aleksandra

Korać, Petra

Korbecki, Jan

Korczeniewska, Olga

Kordyukova, Larisa

Kordyum, Elizabeth L.

Korfei, Martina

Korinek, Vladimir

Korkhov, Volodymyr

Kornhuber, Malte.

Kornke, Alex

Kornmann, Marko

Koromyslova, Anna

Korsching, Eberhard

Korytáŕ, Tomáš

Korzhikova-Vlakh, Evgenia

Korzhikov-Vlakh, Viktor

Kosacka, Joanna

Kosan, Christian

Kosecka-Strojek, Maja

Koshiba, Takumi

Koskinas, John

Koskinen, Pekka

Kośla, Katarzyna

Kosmala, Arkadiusz

Kosman, Joanna

Košmerl, Tatjana

Kosova, Klara

Kosowska, Agnieszka

Kosslick, Hendrik

Kostakis, Ioannis

Koštál, Vladimír

Kostareva, Anna

Kostecka-Gugala, Anna

Kosters, Astrid

Kostner, Gert M.

Kostopoulou, Ourania

Kostova, Dimitrina

Kostrominova, Tatiana

Kostyushev, Dmitry

Kosuge, Yasuhiro

Kosutova, Petra 
Koswara, Andy

Kosyakov, Dmitry S.

Koszelewski, Dominik

Kota, Satya

Kotani, Tomoya

Kotanidou, Anastasia

Kotelevets, Larissa

Kotra, Lakshmi P.

Kotsiou, Ourania S.

Kottakota, Chandrasekhar

Kotta-Loizou, Ioly

Kottaridi, Christine

Koturbash, Igor

Kou, Yanjun

Koukos, Panagiotis I.

Koukoulaki, Maria

Koulen, Peter

Kounatidis, Ilias

Kouril, Roman

Kouroumalis, Elias A.

Kouroupis, Dimitrios

Kourtidis, Antonis

Koutelou, Evangelia

Kouza, Maksim

Kovačević, Borislav

Kovacs, Anita

Kovacs, Lajos

Kovacs, Laszlo

Kovacs, Renato

Kovács-Öller, Tamás

Koval, Alexey

Koval, Michael

Koval, Olga

Kovalchuk, Nina

Kovalenko, Elena I.

Kovaleva, Valentina

Kovalská, Mária

Koven, William

Kovinich, Nik

Kowalewska, Magdalena

Kowalska, Joanna

Kowalska, Karolina

Kowalska-Duplaga, Kinga

Kowol, Christian R.

Kowtharapu, Bhavani S.

Koya, Richard

Koyama, Nobuhiro

Koyama, Yasuhito

Koyama, Yutaka

Koyuncu, Orkide

Koyuncu, Seda
Kozak, Ashot

Kozak, Christine

Kozaki, Akiko

Kozakiewicz, Anna

Kozakiewicz, Mariusz

Kozhevnikova, Oyuna

Koziak, Katarzyna

Kozicz, Tamas

Koziorowska-Gilun, Magdalena

Koziróg, Anna

Kozlov, Konstantin

Kozlowski, Lukasz

Kozlowski, Michael R.

Kozlowski, Piotr

Kozovska, Zuzana

Krabbenhoft, Trevor J.

Kracher, Daniel

Krägeloh-Mann, Ingeborg

Krainer, Georg

Krajewska-Włodarczyk, Magdalena

Krajka-Kuźniak, Violetta

Krajnak, Kristine M.

Krakowski, Leszek

Kramarenko, Elena Yu

Kramer, Boris W.

Krammer, Eva-Maria

Kranenburg, Richard Van

Kranendonk, Michel

Kraner, Susan D.

Kranz, Mathias

Krappmann, Daniel

Krasheninina, Olga A.

Krasniqi, Ahmet

Krásný, Libor

Krasuska, Urszula

Kratochvilova, Irena

Kratochwill, Klaus

Kratzke, Robert Arthur

Krauklis, Andrey E.

Kraus, Allison

Kraus, Dominik

Kraus, Petra

Krause, Gerd

Krause, Günter

Krause, Julia

Kravats, Andrea

Kravets, Volodymyr

Krawczyk, Konrad

Krazinski, Bartlomiej Emil

Krchnak, Viktor

Krčma, František 
Krebs, Joachim

Kreft, Marko

Kregiel, Dorota Maria

Kreimer, Simion

Kreis, Nina-Naomi

Krela-Kaźmierczak, Iwona

Krell, Tino

Křen, Vladimir

Krenek, Peter

Kresoja-Rakic, Jelena

Kress, Michaela

Krestinina, Olga

Kretov, Dmitry

Kretz, Markus

Kreuger, Johan

Krieger-Liszkay, Anja

Krieghoff-Henning, Eva

Krijnse-Ĺocker, Jacomine K.

Krishna, Somashekar G.

Krishnakumar, Shyam

Krishnan, Natraj

Krishnan, Vinu

Kristensen, Mie

Kritchenkov, Andreii S.

Kritsiligkou, Paraskevi

Kriz, Jan

Krizbai, István A.

Kroeker, Andrea

Krogfelt, Karen

Krojer, Tobias

Krokidis, Marios

Krokosz, Anita

Krol, Silke

Królicka, Agnieszka

Królicka, Aleksandra

Kronenberg, Daniel

Kroner-Milsch, Antje

Kropp, Martina

Kroupin, Pavel

Krstic, Jelena

Krug, Sebastian

Krug, Susanne M.

Krüger, Elke

Krüger, Marcus

Kruithof De Julio, Marianna

Krum, Susan A.

Krummenacher, Claude

Krupa-Małkiewicz, Marcelina

Kruse, Rikke

Kruspig, Bjorn

Kruszewski, Marcin
Kruzel, Marian L.

Kryger, Per

Krylov, Sergey N.

Krystyna, Skalicka - Woźniak

Kržan, Mojca

Krzeptowski, Wojciech

Krzyściak, Wirginia

Krzystanek, Marek

Krzystek-Korpacka, Malgorzata

Krzysztof Nowak, Jan

Krzyźowska, MaŁgorzata

Kshirsagar, Prakash G.

Książek, Krzysztof

Książkiewicz, Michał

$\mathrm{Ku}$, Chuan

$\mathrm{Ku}$, Kang-Mo

$\mathrm{Ku}$, Seung-Yup

$\mathrm{Ku}$, Yee Shan

Kuan, Yu-Hsiang

Kuba, Michael

Kubaczka, Caroline

Kubala, Lukáš

Kubala, Szymon

Kubatka, Peter

Kubatzky, Katharina

Kubiak, Katarzyna

Kubicova, Lenka

Kubilius, Raimondas

Kubinová, Šárka

Kubiński, Konrad

Kubinyi, Miklós

Kubisz, Peter

Kubo, Asako

Kubo, Atsushi

Kubo, Eri

Kubo, Yoshinao

Kucera, Radek

Kucerova, Lucie

Kucharíková, Soňa

Kucharska, Karolina

Kuchay, Shafi M.

Kuchler-Bopp, Sabine

Kuçi, Selim

Kucinski, Krzysztof

Kück, Ulrich

Kućko, Agata

Kucuk, Israfil

Kucukoglu, Melis

Kuczera, Piotr

Kudaibergenov, Sarkyt

Kudo, Yasusei 
Kudoyarova, Guzel

Kudryasheva, Nadezhda S.

Kudryashov, Dmitri S.

Kudryavtsev, Denis S.

Kuehne, Thomas

Kues, Wilfried A.

Kufer, T. A.

Kugler, Matthias Christian

Kuhnast, Bertrand

Kuhnert, Eric

Kujan, Omar

Kujawa, Katarzyna

Kujawska, Małgorzata

Kukita, Yoji

Kukull, Walter A.

Kulasinghe, Arutha

Kulcenty, Katarzyna

Külheim, Carsten

Kuliczkowski, Kazimierz

Kulig, Dominika

Kulik, Anna

Kulkarni, Varun

Kulma, Anna

Kulma, Magdalena

Kulminskaya, Anna A.

Kulminski, Alexander

Kulus, Dariusz

Kumai, Takumi

Kumamoto, Eiichi

Kumar, Akhilesh

Kumar, Alok

Kumar, Anuj

Kumar, Arun

Kumar, Ashutosh

Kumar, Brajesh

Kumar, Gaurav

Kumar, Hirdesh

Kumar, Justin

Kumar, K.J. Senthil

Kumar, Naresh

Kumar, Nitin

Kumar, Pijush

Kumar, Prashant

Kumar, Praveen

Kumar, Raj

Kumar, Rajesh

Kumar, Ramesh

Kumar, Santosh

Kumar, Satish

Kumar, Shailesh
Kumar, Sujeet

Kumar, Suneel

Kumar, Ujendra

Kumarasinghe, Sujith Prasad

Wanniarachchi

Kumaraswamy, Chitrala

Kumari, Neelam

Kumari, Sonam

Kume, Kensuke

Kumita, Janet

Kummer, Kai

Kumpulainen, Tatu

Kumru, Baris

Kun, Jozsef

Kundrata, Robin

Kunej, Tanja

Kungl, Andreas

Kunikazu, Tanji

Kunoh, Tatsuki

Kun-Rodrigues, Célia

Kunwar, Sanju

Kunz, Wolfram

Kuo, Cheng-Chin

Kuo, Hann-Chorng

Kuo, Hsiao-Mei

Kuo, Ko-Lin

Kuo, Ming-Tse

Kuo, Pao-Lin

Kuo, Shu-Jui

Kuo, Szu-Cheng

Kuo, Yao-Haur

Kupai, Krisztina

Kupcova Skalnikova, Helena

Küpper, Jan-Heiner

Kuppusamy, Maniselvan

Kurabi, Arwa

Kurakula, Kondababu

Kurano, Makoto

Kurańska, M.

Kurańska, Maria

Kurczyńska, Ewa

Kurg, Reet

Kurganov, Boris I.

Kurien, Biji Theyilamannil

Kurihara, Toshihide

Kurihara, Yukio

Kurita, Hisaka

Kurmasheva, Raushan

Kuroda, Daisuke

Kuroda, Teruo

Kurowska, Marzena 
Kurt, Robert A.

Kurumaddali, Abhinav

Küry, Patrick

Kuryk, Lukasz

Kusaczuk, Magdalena

Kusano, Shuhei

Kusewitt, Donna F.

Kushima, Miki

Kushner, Sidney R.

Kusko, Rebecca L

Kuter, Katarzyna

Kutwin, Marta

Kuure, Satu

Kuwabara, Taku

Kuwahara, Masayasu

Kuwar, Suyog S.

Kuwata, Hiroshi

Kuzhir, Polina

Kuzmanovic, Ljiljana

Kuźmicki, Mariusz

Kuzmin, Elena

Kuznetsova, Irina M.

Kuźniar, Agnieszka

Kuzuya, Akinori

Kvandova, Miroslava

Kwa, Faith A.

Kwack, KyuBum

Kwak, Shin

Kwakowsky, Andrea

Kweon, Oh-Kyeong

Kwiatkowska, Ewa

Kwiecien, Jacek

Kwok, Henry Hang Fai

Kwon, Choon-Tak

Kwon, Hyokjoon

Kwon, Seong Young

Kwon, So Hee

Kwon, Soon Hyo

Kwon, Soon Jae

Kwon, Soon Kil

Kwon, Tae-Hwan

Kwon, Woo-Sung

Kwon, Yong-uk

Kwong, Lawrence N.

Kwong, Yok-Lam

Kwun, Hyun Jin

Kyrgios, Ioannis

Kyunghwan, Kim

L'homme, Laurent

L'Ollivier, Coralie

La Clair, James
La Favor, Justin

La Forgia, Daniele

La Penna, Giovanni

La Rosa, Carmelo

La Rosa, Massimo

La Rosa, Piergiorgio

La Spina, Martina

La Teana, Anna

La Vieille, Sébastien

La Vignera, Sandro

Laakmann, Elena

Labazi, Hicham

Labbé, Jessy

Labbozzetta, Manuela

Labeit, Siegfried

Labi, Verena

Labo, Nazzarena

Laboisse, Christian L.

Labro, Alain

Labrou, Nikolaos

Labruna, Giuseppe

Labrune, Philippe

Labus, Karolina

Lacadena, Javier

Lacal, Juan Carlos

Lăcătușu, Cristina-Mihaela

Lach, Ralf

Lacham-Kaplan, Orly

Lachance, Marc-André

Lachmann, Nico

Lachowicz, Dorota

Lacis, Gunars

Lackie, Peter M.

Lackner, Nina

Lacomme, Christophe

Lacza, Zsombor

Łączkowski, Krzysztof Z.

Lademann, Hanne

Ladomery, Michael R.

Ladoux, Annie

Ladyzynski, Piotr

Laezza, Fernanda

Laffey, John G.

Lafond, Mickaël

Lafont, Elodie

Lafont, Jerome

Lafontaine, Denis

Lafontant, Pascal J.

Lafrenaye, Audrey

Laganà, Bruno

Lagarkova, Maria 
Laghezza, Antonio

Lagoumintzis, George

Lagrange, Jeremy

Laguna, JC

Lagunas, Beatriz

Laher, Ismail

Lahiri, Satadru

Lahiri, Tanaya

Lahlil, Rachid

Lahner, Edith

Lahooti, Hooshang

Lahuta, Lesław Bernard

Lai, Chian-Hui

Lai, Chien-Han

Lai, Chih-Chia

Lai, Chih-Ho

Lai, Chyong-Huey

Lai, Hungcheng

Lai, Jui-Yang

Lai, Junyun

Lai, Kent

Lai, Ming-Derg

Lai, Ping-Shan

Lai, Thung-S

Lai, Wen-Fu

Lai, Xuelei

Lai, Yanhao

Lai, Yen-Chun (Charly)

Lai, Yi-Chen

Lai, YingJu

Lai, Yu-Heng

Lai, Zon Weng

Laine, Mika

Laing, William A

Laizé, Vincent

Lajic, Svetlana

Lakin-Thomas, Patricia L.

Lakk, Mónika

Lakomek, Nils Alexander

Lakota, Jan

Lakshmikuttyamma, Ashakumary

Lalak-Kańczugowska, Justyna

Lall, Gurprit S.

Lalmanach, Gilles

Lalowski, Maciej

Lam, Chi Keung

Lam, Sio-Hong

Lam, Yan

Lamar, John

LaMarca, Babette

Lamas, J. Antonio
Lamatsch, Dunja

Lamb, David

Lamb, Laura

Lamba, Doriano

Lambert, Kelly

Lambova, Sevdalina

Lambrinidis, George

Lambrou, George I

Lamers, Marcelo Lazzaron

Lamm, Monica H.

Lamort, Anne-Sophie

Lampert, Angelika

Lampiasi, Nadia

Lamport, Derek T. A.

Lan, Chou-Chin

Lan, Lan

Lan, Yucheng

Lana, Daniele

Lancel, Steve

Lancella, Laura

Landau, Daniel

Landeros-Weisenberger, Angeli

Landi, Lucia

Landi, Simone

Landi, Vincenzo

Landon, Celine

Landreville, Solange

Landrieu, Isabelle

Landry, Marc

Landsberger, Nicoletta

Lane, Hsien-Yuan

Lang, Di

Lang, Fengchao

Lang, Patricia

Lange, Sigrun

Lange, Tobias

Langelaan, David N.

Langendorf, Christopher

Langer, Gitta Jutta

Langer, Ingrid

Langsjoen, Rose

Lanikova, Lucie

Lankinen, Maria

Lannigan, Joanne

Lanning, Nathan

Lansman, Jeffry B.

Lanza, Giuseppe

Lanzafame, Raymond J

Laoudj-Chenivesse, Dalila

Laoui, Damya

Lappano, Rosamaria 
LaPres, John J.

Lapteva, Maria

Lapucci, Andrea

Larauche, Muriel

Larena, Inmaculada

Largy, Eric

Larionova, Irina

Lark, Daniel

Larkindale, Jane

LaRock, Christopher N.

Laron, Zvi

Larre, Isabel

Larriba, Eduardo

Larsen, Melinda

Larson, Göran

Larussa, Tiziana

Lasagni, Laura

Lascano, Jorge

Lasik-Kurdyś, Małgorzata

Lasko, Paul

Lasoń, Władysław

Lasonder, Edwin

Lassche, Saskia

László, Csambalik

Latajka, Zdzisław

Lateef, Zabeen

Latek, Dorota

Latella, Giovanni

Latendresse, Mario

Latini, Andrea

Latinovic, Olga

Latorre, Eva

Latour, Sylvain

Latowski, Dariusz

Lattanzi, Annalisa

Latunde-Dada, Gladys Oluyemisi

Lau, Adeline

Lau, Allison N.

Lau, Benjamin

Lau, Chungho

Lau, Joseph

Lau, Kin H.

Lau, Wei Ling

Lauf, Peter

Laufs, Patrick

Läuger, Jörg

Laughton, Charles A.

Laura, Rosso

Laurent, Patrick

Lauretani, Fulvio

Lauretta, Rosa
Lauricella, Marianna

Laurin, Jérôme

Lauritano, Dorina

Lauro, Clotilde

Lauro, Davide

Lauronen, Jouni

Laursen, Kristian Bruun

Lauterbach, John $\mathrm{H}$.

Lauwers, Elsa

Lavender, Andrew

Lavista Llanos, Sofía

Lavogina, Darja

Lavrik, Inna

Lavrova, Anastasia I.

Lawas, Lovely Mae F.

Lawson, Charlotte

Lawson, Kim

Lazar, Catalin

Lazarev, Vladimir

Lazarević, Vladimir

Lazaridis, Georgios

Lazaris, Anthoula

Lazaro-Dieguez, Francisco

Lazarowski, Alberto

Lazartigues, Eric

Lazennec, Gwendal

Lazutka, Juozas

Lazzara, Giuseppe

Lazzarato, Loretta

Le Clec'h, Winka

Le Dévédec, Sylvia

Le Guillou, Dounia

Le Huërou-Luron, Isabelle

Le Joncour, Vadim

Le Magueresse-Battistoni, Brigitte

Le Romancer, Muriel

Le Saux, Olivier

Le, Hoang-Thanh

Le, Minh

Leabu, Mircea

Leach, Jennie B.

Leach, Katie

Leão, Pedro N.

Leavesley, David

Lebar, Matthew

Lebaron, Richard

Lebaron, Simon

Lebedev, Igor

Lebedev, Vadim

Lebenthal, Yael

Lebert, Michael 
Lebreton, Stéphanie

Lebrón, José Antonio

LEBRUN, Marc-Henri

Lebrun, Vincent

Lebwohl, Mark G

Lecca, Salvatore

Lechanteur, Anna

Lechel, Andre

Lechner, Bob-Dan

Leclercq, Guy

Leclercq, Loic

Lecona, Emilio

Ledda, Caterina

Ledda, Mario

Ledesma, Ana Estela

Ledesma, Maria Dolores

Ledo, Ana

Lee, An-Rong

Lee, Bong Ho

Lee, Brenda Y.

Lee, Byong-taek

Lee, Byoung Dae

Lee, Chang-Hoon

Lee, Changshing

Lee, Chang-Soo

Lee, ChangWoo

Lee, Chanhui

Lee, Che-Hsin

Lee, Cheng-I

Lee, Chia-Hwa

Lee, Chien-Te

Lee, Chih-Hung

Lee, Ching-Chi

Lee, Ching-Kuo

Lee, Choong-Gu

Lee, Choongho

Lee, Chung-Hao

Lee, Dae Ho

Lee, Dae-Hee

Lee, Darren

Lee, Dong Hun

Lee, Dong Ryul

Lee, Eugene $S$.

Lee, Eun Jung

Lee, Eun Young

Lee, Eun-Woo

Lee, Heedoo

Lee, Heonsoo

Lee, Hiang Kwee

Lee, Hsin-Chen

Lee, Hsinyu
Lee, Hye Young

Lee, Ik Jae

Lee, I-Ta

Lee, Jae Wook

Lee, Jaecheol

Lee, Jeong Wook

Lee, Jeong-Dong

Lee, Jetty Chung-Yung

Lee, Ji Min

Lee, Jin-Yong

Lee, Joo Hyoung

Lee, Joohyung

Lee, Jue-Yeon

Lee, Jun Hee

Lee, Jung Ro

Lee, Jung Seok

Lee, Jung-Kul

Lee, Jungwoo

Lee, Keun-Hyeung

Lee, Kwanuk

Lee, Kyung Jun

Lee, Kyungmin

Lee, Li-Ang

Lee, Li-Jen

Lee, Man Ryul

Lee, Meng-shiou

Lee, Min Young

Lee, Myung-Shik

Lee, Peter A

Lee, Sang Bong

Lee, Sang Jin

Lee, Sangho

Lee, Sangyeop

Lee, Seung Hee

Lee, Seung Hwan

Lee, Seung-Hong

Lee, Seung-Hwan

Lee, Sewon

Lee, Shyi-Long

Lee, Soo In

Lee, Soo Young

Lee, Soo-youn

Lee, Stella

Lee, Sujin

Lee, Sung Ki

Lee, Sung-Jae

Lee, Sun-Young

Lee, Tet Woo

Lee, Tse-Min

Lee, Tzong-Shyuan

Lee, Wei-Hwa 
Lee, Wing-Kee

Lee, Won Hee

Lee, Won-Ha

Lee, Yan

Lee, Yeon Sun

Lee, Yi-Jang

Lee, Yongbok

Lee, YoonJung

Lee, Young Han

Lee, Youngkyun

Lee, Yun Bin

Lee, Yunjong

Lees, Jennifer $S$.

Leese, Henry J.

Lefebvre, Phlippe

Lefebvre, Veronique

Lefevre, Sophie D.

Lefèvre, Thierry

Leff, Todd

Leffler, Jonatan

Lefrancois, Stephane

Legal, Luc

Legembre, Patrick

Leggatt, Graham

Legname, Giuseppe

Legrand, Sylvain

Legras, Antoine

Lehmann, Christian

Lehner, Małgorzata

Lehocký, Marián

Lehotsky, Jan

Lehtonen, Šárka

Lei, Fang

Lei, Kecheng

Lei, Wei

Lei, Zhentian

Leibovitch, Ilan J.

Leibowitz, Julian

Leichtle, Stefan W.

Leifels, Mats

Leiphrakpam, Premila

Leisz, Sandra

Leitão, Jorge H.

Leitão, José M.

Leitch, Heather A.

Leitinger, Birgit

Leitner, Alexander

Leiva-Brondo, Miguel

Leivonen, S.k.

Lejeune, Fabrice

Lek, Angela
Lek, Monkol

Lel Khattabi, Laila

Lelli, Moreno

Lemay, Guy

Lemcke, Heicko

Lemieux, Hélène

Lemli, Beáta

Lemmermann, Niels A W

LeMoine, Allain

Lemon, Christopher

Lemon, Jennifer A.

Lempiäinen, Juha

Lenaz, Giorgio

Lenci, Elena

Lenfant, Françoise

Leng, Roger

Lentini, Laura

Lenzo, Nat

Leo, Alfredo Di

Leo, Chen Huei

Leo, Jack Christopher

Léon, Catherine

Leonard, Stephen

Leonardi, Antonio

Leonardi, Roberta

Leonardi, Rosalia

Leoncini, Giuliana

Leoncini, Lorenzo

Leone, Marilisa

Leone, Philippe

Leonetti, Francesco

Leonhardt, Ralf

Leoni, Alberto

Leon-Juarez, Moises

Leonzino, Marianna

Lepage, Cecile

Lepeduš, Hrvoje

Lepenies, Bernd

Leppik, Liudmila

Lequin, Olivier

Lerat, Emmanuelle

Lermyte, Frederik

Lerner, Aaron

Lerner, Ulf

Lerner, Ulf $\mathrm{H}$.

Leroy, Didier

Les, Francisco

Leshchynska, Iryna

Lesina, Marina

Lesniak, Wieslawa

Lesov, Ivan 
Lessmann, Volkmar

Lestavel, Sophie

Lesuisse, Dominique

Lesyk, Roman B.

Leszczynska, Katarzyna

Letizia, Trovato

Letts, James

Leu, Steve

Leuci, Valeria

Leung, Nicki Y.H.

Leung, Tin-Chung

Leung, Yu min

Levantini, Elena

Levaot, Noam

LeVasseur, Nathalie

Levavi-Sivan, Berta

Levenson, Cathy

Lever, Teresa

Levi Sandri, Giovanni Battista

Levi, Giovanni

Levick, Scott

Levitskii, Sergey

Levitsky, Victor G.

Levy, Arkene S.A.

Levy, Dan

Levy, Steven

Lewandowska, Małgorzata

Lewandowska-Polak, Anna

Lewandowska-Szumieł, Małgorzata

Lewczuk, Bogdan

Lewicki, Sławomir

Lewin, Alfred

Lewin, Ewa

Lewinska, Anna

Lewis, James H.

Lewis, Jo

Lewis, L. Kevin

Lewsey, Mathew G.

Ley, Benedikt

Leybaert, Luc

Lezot, Frederic

Lezza, Angela Maria Serena

Li Volti, Giovanni

Li, Bing-Yan

Li, Chao

Li, Chenshuang

Li, Chia-Jung

Li, Chien-Feng

Li, Chin

Li, Dequan

Li, Dongmei
Li, Dongqing

Li, Dongying

Li, Fengzhi

Li, Gang

Li, Guannan

Li, Guohui

LI, Hai-chang

Li, Haobo

$\mathrm{Li}, \mathrm{He}$

Li, Hongchun

Li, Hongda

Li, Hongjie

Li, Hua

Li, Huinan

Li, Hung-Wen

Li, Jia

Li, Jiao Jiao

$\mathrm{Li}$, Jiashen

Li, Ji-liang

Li, Jinping

Li, Junhao

Li, Junxu

Li, Lei

Li, Lianbo

Li, Lin

Li, Ling

Li, Lingxiao

Li, Liping

Li, Mei-Ling

Li, Min

Li, Ming

Li, Paul C.H.

Li, Pei Feng

Li, Peng

Li, Quanxi

Li, Renfeng

Li, Ronghui

Li, Shengwen Calvin

Li, Shizhao

$\mathrm{Li}$, Shuren

Li, Sunan

Li, Tianhu

Li, Tieshi

Li, WAN-CHUN

Li, Wei

Li, Weihan

Li, Wei-Ming

Li, Wenbo

Li, Wenyi

Li, Xiangnan

Li, Xiaojun 


\begin{tabular}{|c|c|}
\hline Li, Xiaopeng & Lietha, Daniel \\
\hline Li, Xin & Ligaba-Osena, Ayalew \\
\hline Li, Xunde & Ligat, Gaetan \\
\hline Li, Xuping & Lihi, Norbert \\
\hline Li, Yin & Likhatskaya, Galina \\
\hline Li, Ying & Li-Kroeger, David \\
\hline Li, Yinglu & Likus, Wirginia \\
\hline Li, Yongle (Leo) & Lillo, Cathrine \\
\hline Li, Zhi-yong & Lim, Andy K.H. \\
\hline Li, Zhongwei & Lim, Chin Yan \\
\hline Li, Ziru & Lim, Chinten James \\
\hline Li, Zongli & Lim, Chunghun \\
\hline Lia Do Amaral, Sandra & Lim, Daewoon \\
\hline Liakina, Valentina & Lim, Edwin \\
\hline Lian, Qizhou & Lim, Hyungyu \\
\hline Liang, Jun & Lim, Jae Hyang \\
\hline Liang, Pei & Lim, Junxian \\
\hline Liang, Pi-Hui & Lim, Kee Siang \\
\hline Liang, Winnie S. & Lim, Kenji \\
\hline Liang, Zhikai & Lim, Ki-Taek \\
\hline Liao, Chen & Lim, Kyung-Min \\
\hline Liao, Daiqing & Lim, Lee Wei \\
\hline Liao, Jyh-fei & Lim, Megan S. \\
\hline Liao, Wupeng & Lim, Rayne R. \\
\hline Liao, Xudong & Lim, Soon Sung \\
\hline Liao, Yi-Jen & Lim, Soyeon \\
\hline Liao, Yu-Chieh & Lim, Tae-Gyu \\
\hline Liapi, Charis & Lima, Dênis \\
\hline Liarte, Sergio & Lima, Sofia \\
\hline Liatikus, Žilvinas & Lima, Wanessa C. \\
\hline Liau, Nicholas P. D. & Limbach, Patrick \\
\hline Liaw, Chya-Yan & Limon, Agenor \\
\hline Liberi, Giordano & Limozin, Laurent \\
\hline Liberles, David & Lin, Been-Ren \\
\hline Libich, David & Lin, Chang-Chi \\
\hline Libra, Massimo & Lin, Chang-Shen \\
\hline Liby, Karen & Lin, Chia-Hua \\
\hline Licata, Patrizia & Lin, Chieh-Hsin \\
\hline Licciardello, Concetta & Lin, Chien-hong \\
\hline Licciulli, Flavio & Lin, Chih-Chien \\
\hline Lichner, Zsuzsanna & Lin, Chih-Li \\
\hline Licini, Caterina & Lin, Chi-Jan \\
\hline Liebau, Stefan & Lin, Dar-Shong \\
\hline Liebel, Matz & Lin, Hai-Shu \\
\hline Liebman, Michael & Lin, Ho \\
\hline Liebman, Sue W. & Lin, Hui-Hsuan \\
\hline Liebscher, Jürgen & Lin, Hung-Yin \\
\hline Liechti, George W. & Lin, I-Cha \\
\hline Liechti, Matthias Emanuel & Lin, Jian-Ming \\
\hline Liehr, Thomas & Lin, Kuan Hung \\
\hline Liesveld, Jane & Lin, Kwang-Huei \\
\hline
\end{tabular}


Lin, Long-Liu

Lin, Ming-Hong

Lin, Muh-Shi

Lin, Pei-Hui

Lin, Peter P.

Lin, Ruwei

Lin, Shian-Ren

Lin, Shih-Yi

Lin, Shuan-Pei

Lin, Tao

Lin, Tzu-En

Lin, Wei Chieh

Lin, Weifeng

Lin, Wei-Yi

Lin, Wen

Lin, Wey-jinq

Lin, Yanghsiang

Lin, Ya-wen

Lin, Yibin

Lin, Ying-Hung

Lin, Yi-Ting

Lin, Yu-Hsuan

Lin, Yu-Ling

Lin, Zhiwei

Linares-Pastén, Javier

Linciano, Pasquale

Lindahl, Lasse

Lindberg, David R.

Lindberg, Gerrick

Lindeman, Jan

Lindemann, Stephan

Linden, Anni-Maija

Linden, Rafael

Lindenmann, Joerg

Linder, Stig

Lindkvist, Karin

Lindner, Steffen

Lindsay, Andrew J.

Lindseth, Inge

Lindström, Mikael S.

Linhares, Paulo

Linial, Michal

Linke, Dirk

Lins, Laurence

Linseman, Daniel

Liobikas, Julius

Lionetto, Maria Giulia

Lionne, Corinne

Liopis, Juan

Liou, Chian-Jiun

Liou, Gunn-Guang
Liou, Jr-Jiun

Liou, Ying-ming

Liovic, Ivica

Liovic, Mirjana

Lioy, Simone

Lipert, Anna

Lipina, Radim

Lipinski, Daniel M.

Lipiński, Piotr F. J.

Lipke, Peter

Lipparini, Filippo

Lippert, Elisabeth

Lipphaus, Andreas

Lis Arias, Manuel J.

Lis, Grzegorz J.

Lisacek, Frederique

Lisi, Lucia

Lisowska, Katarzyna

Lissa, Delphine

Listos, Joanna

Litak, Jakub

Litschauer, Brigitte

Little, Julian

Little, Marc

Litvinova, Ekaterina A.

Litvinova, Larisa

Litwin, Ireneusz Bernard

Liu, Aimin

Liu, Chao-Lin

Liu, Chaoxing

Liu, Cheuk Lun

Liu, Chi-Ming

Liu, Ching-Ping Liu

Liu, Chung-Jung

Liu, Chunyu

Liu, David

Liu, Der-zen

LIU, FANG

Liu, Gang

Liu, Hengrui

Liu, Hongyu

Liu, Hu-chen

Liu, J. Jay

Liu, Jiang

Liu, Jui-Ming

Liu, Jun

Liu, Ling

Liu, Liyun

Liu, $\mathrm{Lu}$

Liu, Mo

Liu, Ning 
Liu, Pengda

Liu, Qi

Liu, Qinghang

Liu, Qun

Liu, Rong

Liu, Shenkui

Liu, Shih-Ping

Liu, Shing-Hwa

Liu, Shirong

Liu, Tao

Liu, Ting-Yu

Liu, Wei

Liu, Wei-Hsiu

Liu, Wenxian

Liu, Xiaobo

Liu, Xiaojie

Liu, Xiaoying

Liu, Yajun

Liu, Yang

Liu, Yi-Chang

Liu, Yingjun

Liu, Yonggang

Liu, Yongliang

Liu, Yu

Liu, Yu-Chang

Liu, Yu-Peng

Liu, Yutao

Liu, Zhaohui

Liu, Zhi

Liu, Zhipeng

Liu, Zhixia

Liu, Zhongwei

Liu, Ziqing

Liutkevičienè, Rasa

Livanos, Pantelis

Liverani, Liliana

Livingston, David

LiWang, Patricia

Liwinienko, Grzegorz

Lizard, Gérard

Lizcano, Fernando

Lizcano, Jose M.

Lleo, Ana

Llopis-González, Agustín

Llorens Martin, Maria

Llorens, Eugenio

Llorens, Jordi

Llorente, Irene

Lloret, Ana

Lluna, Amparo Gamero

Lo Giudice, Antonino
Lo Giudice, Roberto

Lo Muzio, Lorenzo

Lo Piero, Angela Roberta

Lo Sardo, Federica

Lo, Chunmin C.

Lo, Kai-Yin

Lo, Szecheng

Lobanova, Ekaterina S.

Lobo, Glenn P.

Locascio, Antonella

Locatelli, Giuseppe

Lochhead, Jeffrey J.

Lochmanová, Gabriela

Lochner, Martin

Lockshin, Michael D.

Lodge, Katharine M.

Lodi, Alessia

Lodyga-Chruscinska, Elżbieta

Loebel, Claudia

Loeffler, Ivonne

Loessner, Daniela

Logan, Derek

Loginov, Dmitriy

Lognay, Georges C.

Lohan, Silke

Lohmann, Volker

Lohning, Anna

Lohr, Christian

Loid, Josef

Loiselle, Denis S.

Loizzo, Monica Rosa

Lojkić, Ivana

Lojkova, Lea

Lojou, Elisabeth

Lok, Chun Nam

Loka, Dimitra

Lokensgard, James $\mathrm{R}$

Lokman, Mark

Lokshin, Anna

Lombardi, Assunta

Lombardi, Francesca

Lombardi, Giuseppe

Lombardi, Raffaella

Lombardi, Vincent C.

Lombardo, Giovanni Enrico

Lomovatskaya, Lidia

Lonardo, Amedeo

Lonardo, Enza

Lončar, Mirela Baus

Londei, Paola

London, Sarah E. 
Lonetti, Annalisa

Long, Chiau Ming

Long, Yicheng

Long, Yuchen

Longo, Antonio

Longo, Valentina

Longone, Patrizia

Longy, Michel

Lönnberg, Harri

Lopes, Alberto Jorge Oliveira

Lopes, Graça

López Lluch, Guillermo

López Malo, Daniel

López Martínez, Juan

Lopez Martinez, Maria José

López Nicolás, Jose Manuel

López Sesé, Ana Isabel

Lopez, César A.

López, Cristina

Lopez, Jose Cristobal

Lopez, Jose Javier

López, Luis Carlos

Lopez, Sophie

López-Camarillo, César

Lopez-Canul, Martha

Lopez-Castejon, Gloria

López-Cebral, Rita

Lopez-Escamez, Jose A.

Lopez-Ferber, Miguel

López-Gallego, Fernando

López-Guerrero, José Antonio

López-Hernández, Luz Berenice

López-Hernández, Yamilé

López-Huertas, María Rosa

López-López, Daniel

López-López, Elixabet

López-López, Manuel

López-Marqués, Rosa Laura

López-Pintor Muñoz, Rosa María

López-Ruiz, Elena

Łopieńska-Biernat, Elżbieta

Loppi, Stefano

Loppi, Steffano

Łopusiewicz, Łukasz

Lora, Jorge

Lorberboum-Galski, Haya

Lordan, Ronan

Lordkipanidzé, Marie

Lorenz, Alexander

Lorenz, Kristina

Lorenz, Ronny
Lorenz, Susanne

Lorenzo González, Óscar

Lorenzo Sanchez, Oscar

Lorenzo, Sempere

Loreti, Elena

Lorite, Pedro

Losada-Perez, Patricia

Loschenov, Victor B.

Loskog, Angelica

Lossius, Andreas

Losurdo, Giuseppe

Łotocka, Barbara

Lotti, Lavinia Vittoria

Louis, Sandrine

Loura, Luís M.S.

Lourenço, Tiago

Louveau, Antoine

Lovat, Francesca

Lovato, Laura

Lovatt, Matthew

Lovisa, Sara

Lovisolo, Claudio

Löw, Péter

Lowe Jr., William L.

Lowe, Gillian

Lowe, Martin

Lowery, Jonathan

Lowin, Torsten

Lozano, R. M.

Lozano-Elena, Fidel

Lozano-Lorca, Macarena

$\mathrm{Lu}$, Ailing

Lu, Chih-Hao

$\mathrm{Lu}, \mathrm{Chi}-\mathrm{Yu}$

Lu, Chung-kuang

Lu, Da-Wen

$\mathrm{Lu}$, Hong

Lu, Hong S.

$\mathrm{Lu}$, Jing

Lu, Kuo-Cheng

Lu, Linchao

Lu, Lixia

Lu, Ming-Wei

Lu, Pei-Luen

Lu, Quanlong

Lu, Sizhao

Lu, Wei

Lu, Wei-Yu

$\mathrm{Lu}, \mathrm{Xiao}$

Lu, Yang

$\mathrm{Lu}$, Yangfan 


\author{
Lu, Yongke \\ $\mathrm{Lu}$, You \\ Lu, Yubing \\ Lu, Yujiao \\ Lu, Zhanping \\ Lualdi, Marta \\ Lubawy, Jan \\ Lubecka-Pietruszewska, K \\ Lubell, William D. \\ Luca, Andrei \\ Luca, Giovanni \\ Luca, Maria \\ Luca, Rossella \\ Lucaciu, Constantin Mihai \\ Lucantoni, Federico \\ Lucarelli, Enrico \\ Lucas, Heather R. \\ Lucas, Jose Antonio \\ Lucchese, Alessandra \\ Lucchetti, Donatella \\ Lucena, Carlos \\ Lucenti, Elena \\ Lucero, Diego \\ Luchetti, Andrea \\ Luchetti, Michele M. \\ Luchian, Tudor \\ Luchinat, Claudio \\ Luchini, Claudio \\ Lucic, Vladan \\ Lucioli, Simona \\ Luck, Rudy \\ Luckman, Simon \\ Luddi, Alice \\ Luddy, Kimberly A. \\ Ludidi, Ndiko \\ Ludlow, Andrew T. \\ Ludwig, Andreas \\ Ludwig-Müller, Jutta \\ Ludwikõw, Agnieszka \\ Lue, Ko-Huang \\ Luebbert, Christian \\ Luengo, Yurena \\ Luethi, Dino \\ Lugnier, Claire \\ Ługowska, Agnieszka \\ Lui, Kathy \\ Lukacher, Aron E. \\ Lukas, Jan \\ Łukasiuk, Katarzyna \\ Lukaszewski, Adam J. \\ Łukomska, Agnieszka
}

Łukowski, Adrian

Lukyanov, Konstantin A.

Luna, Belén

Lund, Elizabeth K.

Lundborg, Magnus

Luneau, Dominique

Lung, Shiu Cheung

Lungaro, Lisa

Lunov, Oleg

Lunt, Peter

Luo, Chi-Wen

Luo, Lin

Luo, Minkui

Luo, Ray

Luo, Weibo

Luongo, Livio

Lupien, Andréanne

Lupini, Antonio

Lupu, Stelian

Luque, Francisco

Lurz, Eberhard

Lusa, Merja

Lushington, Gerry

Lustgarten, Michael

Lustig, Arthur

Lustig, Yaniv

Łuszczki, Jarogniew

Lüthje, Sabine

Lutter, Erika

Lüttge, Ulrich E.

Lutz, Pierre-Eric

Luuk, Hendrik

Luvisi, Andrea

Luzio, Ana

Luznik, Zala

Lymperopoulos, Anastasios

Lyu, Ping-Chiang

Lyubchenko, Yuri

Lyukmanova, Ekaterina N.

M'kacher, Radhia

Ma, Guojia

Ma, Hong

Ma, Jiyan

Ma, Lisha

Ma, Peisong

Ma, Qianli

Ma, Suk Ling

Ma, Wanshu

$\mathrm{Ma}$, Wei

$\mathrm{Ma}$, Xiaochao

Ma, Xiaochi 
Ma, Yiyi

Maak, Steffen

Maass, Kendra

Maaß, Sandra

Mabbott, Neil

Mabbott, Samuel

Maccallini, Cristina

Maccari, Alberto

Maccarinelli, Federica

Macchi, Samntha

MacDonald, Clinton C.

Macedo, Antónia

Macek Jilkova, Zuzana

Macek Jílková, Zuzana

MacEwan, David

Mach, Mojmir

Machado, Raul

Machairiotis, Nikolaos

Machelart, Arnaud

Machetti, Fabrizio

Machon, Ondrej

Machuca-Gayet, Irma

Machuca-Portillo, Guillermo

Macías, Ramón

Maciej, Strzemski

Maciejewska, Barbara

Maciejewski, Ryszard

Macín, Stella Maris

Macirella, Rachele

Maciver, Bryce

MacIver, M. Bruce

Mackiewicz, Andrzej

Mackiewicz, Dorota

MacNeill, Amy

Maçôas, Ermelinda

Macori, Guerrino

MacRaild, Christopher

Mączka, Wanda

Madak-Erdogan, Zeynep

Madala, Satish

Madaro, Luca

Madeddu, Paolo

Madeddu, Roberto

Madeira, Maria H.

Madej, Dawid

Madej, M. Gregor

Maden-Wilkinson, Thomas

Mádi, András

Madigan, James P.

Madka, Venkateshwar

Madkour, Aicha
Mädler, Kathrin

Madonna, Stefania

Madonov, Pavel

Madrid, Eva

Madsen, Claus Krogh

Madueño, Rafael

Madurantakam, Parthasarathy A.

Madureira, Patrícia A.

Maduro, Morris

Maeda, Akiko

Maeda, Hiroshi

Maeda, Kazuhiro

Maeda, Megumi

Maeda, Yuichi

Maehre, Hanne

Maekawa, Ryo

Maekawa, Shun

Maenaka, Katsumi

Maestri, Elena

Maestú Unturbe, Ceferino

Maffei, Margherita

Maftah, Abderrahman

Magdinier, Frederique

Maggert, Keith

Maggi, Davide

Maggo, Simran

Magherini, Francesca

Maghuly, Fatemeh

Magierowski, Marcin

Magi-Galluzzi, Cristina

Magin, Thomas M.

Magini, Alessandro

Magistrato, Alessandra

Maglio, Cristina

Maglio, Melania

Maglio, Ornella

Magnaghi, Valerio

Magnaldo, Thierry

Magnani, Corrado

Magnani, Francesca

Magnani, Mauro

Magnano Di San Lio, Gaetano

Magne, David

Magnus, Marcin

Magnusson, Karl-Eric

Magrassi, Lorenzo

Maguire, Donogh

Mahalingaiah, Prathap

Mahalingam, Ramamurthy

Mahato, Neelima

Mahavadi, Sunila 
Mahéo, Karine

Maher, Pamela

Mahmood, Iftekhar

Mahmood, Khalid

Mai, Hans-Jörg

Maia, Cláudio

Maidaniuc, Andreea

Maier, Camelia

Maier, Patrick

Maillard, Jean-Yves

Maimets, Toivo

Mainardi, Marco

Mainen, Zachary F.

Maioli, Margherita

Maioli, Silvia

Maione, Angela Serena

Maione, Francesco

Maiorano, Domenico

Maiorano, Eugenio

Maiorino, Matilde

Maitarad, Phornphimon

Maitra, Radhashree

Maity, Sankar

Maity, Shuvadeep

Maiuolo, Jessica

Maixent, Jean Michel

Maj, Malgorzata

Majd, Nazanin

Májek, Pavel

Majello, Barbara

Majer, Anna

Majera, Dusana

Majerova, Tatana

Majeský, Luboš

Majewska, Anna

Majewski, Lukasz

Maji, Tanmoy

Majima, Hideyuki J.

Majkowska-Skrobek, Grażyna

Major, Ian

Major, Ian T.

Majtan, Juraj

Majumdar, Rajtilak

Majumder, Mousumi

Majumder, Poulami

Majumder, Pritha

Majzoub, Ahmad

Mak, Ivan

Mak, Lung-Yi

Makabe, Koki

Makarem, Nour
Makarevich, Pavel

Makarevitch, Irina

Makarewicz, Wojciech

Makarova, Katerina

Maki, Kevin

Maki, Masatoshi

Makino, Akiko

Makino, Hiroshi

Makishima, Makoto

Makiyama, Takeru

Maksimovic, Ivana

Maksymiak, Magdalena Martinka

Maksymiuk, Andrew W.

Makunin, Alex

Makunin, Alexey I.

Makvandi, Pooyan

Malacarne, Giulia

Malafa, Mokenge

Malafoglia, Valentina

Malaguarnera, Lucia

Malaguarnera, Michele

Malandrakis, Emmanouil

Malandrino, Andrea

Malanga, Gerard

Malara, Alessandro

Malarkannan, Subramaniam

Malashicheva, Anna

Malaspina, Patrizia

Malavasi, Veronica

Malcher, Agnieszka

Malchik, Fyodor

Maldonado-Contreras, Ana

Malemud, Charles J.

Maléth, József

Maletínská, Lenka

Malewski, Tadeusz

Malfa, Giuseppe Antonio

Malfatti, Dr. Edoardo

Malfatti, Edoardo

Malfeito Ferreira, Manuel

Malhão, Fernanda

Mali, Vishal

Malik, Anna R.

Malik, Taimur

Malik-Gajewska, Magdalena

Malinge, Jean-Marc

Malinowska, Barbara

Malkemper, Pascal

Malladi, Anish

Mallamace, Domenico

Mallela, Shamroop Kumar 
Malli, Roland

Malliavin, Thérèse

Mallipeddi, Prema Latha

Mallipeddi, Srikrishnan

Mallis, Panagiotis

Mallo, Natalia

Málnási-Csizmadia, András

Maloberti, Alessandro

Malouf, Camille

Malpeli, Giorgio

Maltecca, Francesca

Malva, João O.

Maly, Pavel

Malý, Petr

Malyarenko, Timofey V.

Małysz - Cymborska, Izabela

Man, Simona

Manakhov, Anton

Manaskova-Postlerova, Pavla

Manavalan, Balachandran

Manca, Gabriele

Manca, Maria Letizia

Manchia, Mirko

Mancinelli, Romina

Mancini, Annamaria

Mancini, Maicol

Mancini, Stéphane J. C.

Manco, Licínio

Mancuso, Francesca

Mancuso, Salvatrice

Manda, Gina

Mandal, Amritlal

Mandal, Mihir Kumar

Mandal, Mrinmay

Mandalà, Maurizio

Mändar, Reet

Mandard, Stéphane

Mandava, Nagabhushanam Bhushan

Mandelli, Davide

Mandoki, Mira

Mandrioli, Roberto

Mandyam, Chitra

Manenti, Antonio

Manera, Maurizio

Manfredi, Kirk P.

Mang, Stefania Mirela

Mangalagiu, Ionel

Mangani, Stefano

Mangano, Katia

Mangaonkar, Abhishek

Mangiagalli, Marco
Mangino, Giorgio

Mango, Lucio

Mangogna, Alessandro

Mani, Govindasamy

Mani, Sridhar

Maniam, Subashani

Manickavelu, Alagu

Mankin, Alexander

Manley, Sharon J.

Manna, Ida

Mannell, Hanna

Mannello, Ferdinando

Männer, Jörg

Mannik, Jaan

Mannini, Benedetta

Mansier, Olivier

Mansky, Kim C.

Mansour, Sahar

Månsson, Alf

Manstein, Dietmar

Mansuri, Shahid

Mantadakis, Elpis

Mantareva, Vanya N.

Manti, Sara

Manto, Mario

Mantovani, Alberto

Mantripragada, Venkata P.

Manukhina, Eugenia

Manukjan, Georgi

Manville, Rian

Manzo-Merino, Joaquin

Manzotti, Gloria

Mao, Xiaobo

Mapelli, Lisa

Maple, Peter

Maqoud, Fatima

Maraldi, Tullia

Maramai, Samuele

Marampon, Francesco

Maran, Uko

Maranesi, Margherita

Marascio, Nadia

Marasco, Daniela

Marasek-Ciolakowska, Agnieszka

Marassi, Francesca

Marazziti, Daniela

Marć, Małgorzata Anna

Marcel, Virginie

Marcelli, Augusto Claudio

Marcenaro, Emanuela

Marchand, Christophe 
Marchand, Patrice A.

Marchese, Adriano

Marchese, Cinzia

Marchese, Enrico

Marchese, Maria

Marchesi, Isabella

Marchetti, Fabio

Marchetti, Philippe

Marchetti, Sandrine

Marchiani, Sara

Marchini, Antonio

Marchini, Cristina

Marchiò, Caterina

Marcilla, Antonio

Marcinkowska, Ewa

Marco, Francisco

Marco-Contelles, José

Marco-Contelles, Jose Luis

Marcon, Caroline

Marconi, Giovanni

Marconi, Guya Diletta

Marcos, Jose F.

Marcu, Loredana

Marcus, Wieder

Marcuzzo, Stefania

Maréchal, Jean-Didier

Marečková, Klára

Marei, Hany

Marek, Kieliszek

Marek-Trzonkowska, Natalia

Marengo, Barbara

Mareri, Lavinia

Maresca, Vittoria

Mareschi, Katia

Maret, Wolfgang

Maretzky, Thorsten

Margaglione, Maurizio

Margaritis, Lukas

Margassery, Lekha Menon

Margheritis, Eleonora

Margiotta, Azzurra

Margulies, Barry

Marí, Montserrat

Maria Angela, Angela

Maria Carmela, Bonaccorsi Di Patti

Maria Irene, Bellini

Maria Musarella, Carmelo

María Rosaura, Leis Trabazo

Mariadason, John

Marian, Brigitte

Mariano, Caratozzolo
Mariconda, Annaluisa

Mariggiò, Maria Addolorata

Marijuán, Pedro

Marín, Clara

Marin, Jose J.G.

Marin, Luminita

Marín, Macarena

Marincs, Ferenc

Marinelli, Enrico

Marini, Francesca

Marini, Marina

Marino Gammazza, Antonella

Marino, Angela

Marino, Franca

Marino, Joseph

Marino, Tiziana

Mariot, Virginie

Mariotto, Elena

Mariscalco, Giovanni

Mariuzza, Roy A.

Markey, Michael P.

Markiewicz, Aleksandra

Markkanen, Enni

Markopoulos, Anastasios

Markopoulos, Georgios S.

Markopoulou, Olga

Markova, Svetlana

Markova-Car, Elitza P.

Markowicz-Piasecka, Magdalena

Markowitz, Joseph

Marks, Hendrik

Marks, Jeremy D.

Markus, Waldeck-Weiermair

Markwardt, Fritz

Marlicz, Wojciech

Marminon, Christelle

Mármol, Ines

Maron, Steven B.

Marongiu, Mara

Maroni, Paola

Marote, Ana

Marotte, Hubert

Marques, Isabel

Marques, João Gama

Marques, Natália

Marques, Tania

Marquez, Edgar

Marra, Alberto

Marracci, Silvia

Marrano, Nicola

Marrocchi, Assunta 
Marrone, Giuseppe

Marrone, Oreste

Marsano, René Massimiliano

Marsat, Gary

Marschall, Hanns-Ulrich

Marschall, Manfred

Marsolais, Frederic

Marston, Denise

Marston, Steven B.

Martău, Adrian Gheorghe

Martel, Bernard

Martel, Paulo

Martell, Kevin

Martella, Daniele

Martella, Elisa

Martella, Giuseppina

Martelli, Alberto Maria

Martelli, Fabio

Martens, Gerard

Martens, Jürgen

Martens, Stefan

Marteyn, Antoine

Martí, Joaquín

Marti, Matteo

Martí, Sergio

Martial, Sonia

Martikainen, Riikka

Martin Galan, Aida

Martin Miguel, Casco Robles

Martin, Andrew V.

Martín, Carmen

Martin, Daniel

Martin, Elizabeth M.

Martin, Franck

Martin, Keith

Martin, Krssak

Martín, Margarita

Martin, Maria

Martin, Olivier C.

Martin, Patricia

Martin, Ruth

Martin, Sally

Martin, Tammy M.

Martin, Tiphaine

Martina, Matteo

Martín-Antonio, Beatriz

Martín-Aragón, Sagrario

Martín-Cano, Francisco Eduardo

Martín-de-Llano, José Javier

Martinelli, Chiara

Martinelli, Giovanni
Martinez Alonso, Marta

Martinez Calejman, Camila

Martínez De Pablos, Rocío

Martínez Iglesias, Olaia

Martinez, Agustin

Martínez, Alberto

Martínez, Carlos M.

Martinez, Claudia

Martínez, Constantino

Martínez, Isidoro

Martinez, Jose C

Martinez, Luis A.

Martinez, Manuel

Martínez, Marta

Martínez, Miguel A.

Martínez-Abarca, Francisco

Martínez-Arnau, Francisco Miguel

Martínez-Banaclocha, Marcos Arturo

Martínez-Cifuentes, Maximiliano

Martinez-de-Tejada, Guillermo

Martínez-Espinosa, Rosa María

Martínez-Esteso, María José

Martínez-Galiano, Juan Miguel

Martínez-García, Manuel

Martínez-Gómez, Pedro

Martinez-Guryn, Kristina

Martinez-Hervas, Sergio

Martinez-Martos, Jose Manuel

Martinez-Moreno, Carlos

Martinez-Navarrete, Gema

Martinez-Pastor, Felipe

Martínez-Rodríguez, Alejandro

Martínez-Rodríguez, Sergio

Martínez-Salas, Encarnación

Martínez-Sánchez, Noelia

Martinez-Useros, Javier

Martín-Hidalgo, David

Martini, Claudia

Martinka, Michal

Martínková, Ludmila

Martin-Lopez, Eduardo

Martín-Molina, Alberto

Martín-Montalvo, Alejandro

Martín-Nieto, José

Martino, Piera Anna

Martinotti, Simona

Martín-Peláez, Sandra

Martín-Rodríguez, Alberto J.

Martins Lima, Augusto

Martins, Antonio Henrique

Martins, Filipa 
Martins, Ian

Martins, José A.

Martins, Teresa Vaz

Martín-Sánchez, Esperanza

Martín-Satué, Mireia

Martinuzzi, Andrea

Martín-Valero, María Jesús

Martín-Vasallo, Pablo

Marto, Carlos Miguel

Martorana, Alessandro

Martu, Maria Alexandra

Martz, Francoise

Maruani, Antoine

Marumo, Takeshi

Marunaka, Yoshinori

Maruyama, Ichiro

Maruyama, Kei

Marwitz, Sebastian

Marx, Florentine

Marx, Gerald R.

Marx, Joannes J. M.

Marycz, Krzysztof

Marzano, Flaviana

Marzec, Michal

Marzocco, Stefania

Marzoli, Francesca

Masahiro, Sato

Masaki, Takayuki

Masana, Luis

Masarone, Daniele

Masatake, Kanai

Mascarenhas, Mariola

Mascellino, Maria Teresa

Masciarelli, Silvia

Mascini, Macello

Maserti, Bianca Elena

Más-Estellés, J.

Mashiguchi, Kiyoshi

Masi, Annalisa

Masli, Sharmila

Maslow, Joel N.

Mason, Nigel

Masonbrink, Rick

Masquida, Benoit

Massa, Annamaria

Massa, Daniele

Massa, Valentina

Massad, Salwa G.

Massai, Rossano

Massardier, Valérie

Massaro, Marika
Massimiliano, Cordaro

Massimini, Marcella

Massimino Cocuzza, Giuseppe

Massing, Ulrich

Massotte, Dominique

Massoud, Salah

Masternak, Joanna

Masters, Colin L.

Mastinu, Andrea

Mastrangelo, Anna M.

Mastrangelo, Stefano

Mastroleo, Felice

Mastronuzzi, Angela

Masuda, Kiyoshi

Masuda, Mari

Masuda, Takaaki

Masuda, Tatsuru

Masui, Kenta

Masullo, Mariorosario

Masutani, Mitsuko

Masuyer, Geoffrey

Matacchione, Giulia

Matafome, Paulo

Matarredona, Esperanza R.

Matarrese, Paola

Maté, Bélen

Matei, Alina

Matei, Cristian

Matencio, Adrián

Matera, Carlo

Mateus, Maria L.

Mathew, Shibin

Mathias, Clinton

Matiadis, Dimitrios

Matiadis, Dimitris

Matias, Pedro M.

Matikonda, Siddharth

Matilla, Angel J.

Matilla, Miguel

Matlawska-Wasowska, Ksenia

Matoba, Keiichiro

Matoba, Shogo

Matos, Manuela

Matosiuk, Dariusz

Matoušek, Jaroslav

Matraszek-Gawron, Renata

MATRICARDI, Pietro

Matrone, Carmela

Matschke, Johann

Matsubara, Kazuki

Matsubara, Keiichi 
Matsubara, Shigeki

Matsuda, Hisashi

Matsuda, Koichi

Matsuda, Tomoki

Matsuda, Yudai

Matsugaki, Aira

Matsui, Akihiro

Matsui, Hidenori

Matsumoto, Ken

Matsumoto, Takayuki

Matsumoto, Yukihiro

Matsumura, Kiyoshi

Matsumura, Shigeru

Matsumura, Tsuyoshi

Matsunami, Masatoshi

Matsuo, Koichi

Matsuo, Muneaki

Matsuo, Takuya

Matsuo, Tatsuhito

Matsuo, Yasumitsu

Matsuoka, Daisuke

Matsuoka, Isao

Matsushita, Taku

Matsuura, Bunzo

Matsuya, Yusuke

Matsuzaki, Shinya

Matsuzaki, Toshiyuki

Matta, Csaba

Mattapally, Saidulu

Matte, Alessandro

Mattei, César

Mattei, Fabrizio

Matter, Karl

Matteucci, Claudia

Matthew, Benesch

Matthiesen, Rune

Mattii, Letizia

Mattila, Sari

Mattioli, Anna Vittoria

Mattioli-Belmonte, Monica

Mattison, Chris P.

Mattoo, Autar

Mattoscio, Domenico

Mattsson, Mats-Olof

Matucci-Cerinic, Marco

Matulić, Maja

Maturana, Andrés Daniel

Maturana, Carola J.

Matusik, Paweł

Matwijczuk, Arkadiusz

Mátyás, Gábor
Matynia, Anna

Matysik, Silke

Maue, Robert A.

Maugeri, Grazia

Mauhin, Wladimir

Maurer, Barbara

Maurer, Jochen

Mauri, Emanuele

Mauriello, Alessandro

Mauriello, Emilia

Maurizio, Martini

Mauro, Adolfo Gabriele

Mauro, Annunziata

Maury, Stéphane

Maurya, Shailendra

Maver, Tina

Maves, Lisa

Mavridis, Konstantinos

Mavrogeni, Sophie

Mavrogonatou, Eleni

Mavropoulos, Athanasios

Mawhinney, Thomas

Maxim, Aurel

Maxwell, Jessie R.

May, Kerrie L.

Mayan, Maria D.

Maybeck, Vanessa

Mayer, Rupert

Mayer, Thomas

Mayerhofer, Artur

Maynard, Jennifer

Mayr, Johannes A.

Mazan, Sylvie

Mazerska, Zofia

Mazierski, Paweł

Mazilu, Laura

Mazin, Pavel

Mazor, Ohad

Mazur, Antonina Joanna

Mazur-Bialy, Agnieszka

Mazur-Marzec, Hanna

Mazurov, Dmitriy V

Mazzanti, Michele

Mazzeo, Giuseppe

Mazzeo, Maria Fiorella

Mazzoni, Maria

Mazzucotelli, Elisabetta

Mazzuoli-Weber, Gemma

Mbagwu, Smart Ikechukwu

Mc Auley, Mark T.

McArdle, Stephanie 
McBride, Devin W.

McCann, Liza J.

McCann, Matthew R.

McCarthy, John

McCarthy, Michael

McCartney, Fiona

Mccarty, Nael A.

McClung, C. Robertson

McCluskey, Lynnette

McCombe, Pamela

McCorvy, John D.

Mccown, Phillip

McCoy, Annette

McCoy, Sara S.

McCubbin, Andrew

McDaneld, Tara G

McDaneld, Tara G.

McDermott, Suzanne

McDevitt, Christopher

McDonald, Denise

McDonnell, Alison

Mcdonnell, Thomas

McEwan, Iain

McGee-Lawrence, Meghan

McGill, Jodi

McGill, Mitchell R.

Mcgilvray, Kirk C

McGregor, Neil

McGrew, Mike

McIntosh, Robert

McKay, Brian

McKay, Tina

McKenna, Joseph F.

McKenzie, Brienne A.

McKeown-Longo, Paula J.

McKillop, A. M.

McKinstry-Wu, Andrew

McLaren, James E.

McLaurin, JoAnne

McLean, Gary

McManus, Kirk

McMillin, Matthew

McMurray, Cynthia T.

McNally, Martin A.

McPeek, Ashley

McPhillie, Martin

McQuade, Rachel

McRobb, Lucinda S

Meade, Aidan

Meana Paneda, Ruben

Meana, Clara
Mears, Jason

Mechoulam, Raphael

Mechrez, Guy

Mechulam, Yves

Męczyńska-Wielgosz, Sylwia

Meder, Lydia

Mediero, Aránzazu

Medina, David

Medina, Diego

Medina, Milagros

Medina, Reinhold J.

Medina-Kauwe, Lali K.

Medina-Ramírez, Iliana

Medina-Rodríguez, Eva M.

Mediouni, Sonia

Medová, Michaela

Medronho, Bruno Filipe Figueiras

Medvecz, Márta

Medvedev, Ilya Nikolaevich

Meegan, Jamie E.

Meerson, Ari

Megaw, Roly

Mège, René-Marc

Mehaffy, Carolina

Mehariya, Sanjeet

Mehdi, Ebrahimi

Mehla, Jogender

Mehlmann, Lisa

Mehlotra, Rajeev K.

Mehrabadi, Mohammad

Mehrazma, Banafsheh

Mehrpour, Maryam

Mehta, Gaurav A.

Mehta, Gautam

Mehta, Gunjan

Méhul, Bruno

Mei, Kuo-Ching

Mei, Shenglin

Mei, Ya-Fang

Mei, Yang

Meier, Carola

Meierhofer, David

Meijer, Harold J G

Meijers, Björn

Meijers, Wouter

Meijles, Daniel N.

Meimberg, Harald

Meimoun, Patrice

Meinander, Annika

Meinhardt, Matthias

Meiri, Hamutal 
Meisel, Hans-Jörg

Mejuto, Juan

Meksem, Khalid

Melas, Marilena

Melcher, Karsten

Melchor, Juan

Meldolesi, Jacopo

Mele, Andrea

Meleady, Paula

Meléndez-Hevia, Enrique

Melgar, Silvia

Meli, Vijaykumar

Mellett, Mark

Mellidou, Ifigeneia

Melnik, Daniela

Melo, Bernardete F.

Melo, Sonia A.

Meloni, Bruno

Meloto, Carolina Beraldo

Melrose, James

Melve, Guro Kristin

Menasché, Philippe

Mencarelli, Monica

Mendes, Alexandrina Ferreira

Mendes, Livia P.

Mendes, Marta V.

Méndez, Ana

Méndez-Barbero, Nerea

Mendonça, Marcelo

Menegazzi, Marta

Meneses, Carlos

Meneses, Maria João

Menezes, José C. J. M. D. S.

Menezes, Miriam-Rose

Menezo, Yves

Meng, Dong

Meng, Huanxin

Meng, Jinhong

Meng, Qinghang

Meng, Qingshi

Mengel, David

Menin, Chiara

Menke, André

Menon, Manoj B.

Mensah-Nyagam, Guy

Menziani, Maria Cristina

Meola, Giovanni

Meraviglia, Viviana

Mercati, Francesco

Mercedes, Gimeno

Merches, Katja
Merdes, Andreas

Merfort, Irmgard

Mergeay, Max

Mergler, Stefan

Merhi, Ahmad

Merida, Isabel

Merigo, Flavia

Merino, Beatriz

Merino, Jaime M.

Merlot, Elodie

Meroni, Marica

Merrick, Daniel

Mertens, Daniel

Mertlíková-Kaiserová, Helena

Mertz, Janet E.

Merz, Alexey J.

Mesaros, Clementina A.

Mesaros, Eugen

Meshram, Chetan

Mešić, Aleksandar

Meškys, Rolandas

Messina, Samantha

Messori, Luigi

Mestan, Karen

Mester, Patrick-Julian

Mesuraca, Maria

Metwally, Mayada

Metzger, Dennis W.

Metzger, Jochen

Metzger, Monika L.

Metzler, Rebecca

Meurer, Steffen Klaus

Meurette, Olivier

Mevissen, Tycho

Meyer Zu Heringdorf, Dagmar

Meyer, Michel

Meyer, Ralph G

Meyer-Ficca, Mirella L.

Mező, Gábor

Mezzasoma, Letizia

Mezzomo Collares, Fabrício

Mia, Sobuj

Miao, Lingling

Miazek, Arkadiusz

Micale, Nicola

Miceli, Alessandro

Miceli, Vitale

Michael, Adrian

Michaelis, Martin

Michaelson, Daniel

Michaeu, Olivier 
Michal, Kohout

Michalak, Marcin

Michalke, Bernhard

Michalopoulos, Efstathios

Michard, Erwan

Michel, Jean-baptiste

Michel, Martin Christian

Michel, Maximilian

Michel, Piotr

Michel, Sebastian

Michelacci, Yara M.

Micheletti, Gabriele

Micheletti, Rudi

Michelhaugh, Sharon Kay

Michelle, Palmieri

Michels, Alexander

Michelucci, Alessandro

Michelucci, Antonio

Michetti, Fabrizio

Michiels, Chris

Michinaga, Shotaro

Michl, Patrick

Micillo, Raffaella

Micucci, Matteo

Middei, Silvia

Miękus, Natalia

Mielańczyk, Anna

Mielcarek, Michal

Miele, Adriana Erica

Mielgo-Ayuso, Juan

Mierek-Adamska, Agnieszka

Mierzwiński, Józef

Mifsud, Karen

Migdalis, Ilias

Miggiano, Riccardo

Migita, Satoshi

Miglinas, Marius

Migliore, Alberto

Migliori, Edoardo

Mignatti, Paolo

Miguel, Celia

Miguel, Maria Da Graça Costa

Míguez, Jesús M.

Mihály, Boros

Mihály, Judith

Mihara, Keichiro

Mihola, Ondrej

Mikaelyan, Arsen S.

Mikaia, Anzor

Mikala, Gabor

Mikami, Koji
Mikami, Yoshinori

Mikecz, Katalin

Mikelis, Constantinos

Miki, Hiroaki

Miko, Edit

Mikołajczak, Krzysztof

Mikstacka, Renata

Mikula, Mario

Mikulski, Dawid

Mikuš, Peter

Milanese, Marco

Milano, Giuseppina

Milano, Marianne

Milano, Serena

Milanova, Aneliya

Milardi, Danilo

Milatz, Susanne

Milavetz, Barry

Milc, Justyna

Milcarek, Christine

Milczarek, Małgorzata

Milczarski, Pawel

Mildner, Michael

Milella, Luigi

Milenkovic, Dragan

Milenkovic, Vladimir M.

Miles, Wayne Owen

Milescu, Lorin

Miletín, Miroslav

Miletta, Maria

Milhano Santos, Fátima

Milhinhos, Ana

Militello, Giuseppe

Miljković, Miloš D.

Millar, Ann

Millard, Andrew D.

Millecamps, Magali

Miller, Charles

Miller, Dennis

Miller, Elżbieta

Miller, Ingrid

Miller, Justin

Miller, Karl

Miller, Kevin

Miller, Mark W.

Miller, Rachel

Miller, Robert H.

Miller, Stephen C.

Miller, Vandana

Miller, W. Todd

Miller, William B. 
Millet, Óscar

Millon, Laurence

Mills, Ken

Milosavljević, Aleksandar

Miloso, Mariarosaria

Milovanovic, Dragomir

Milroy, Jeffrey J.

Mimura, Junsei

Mimura, Kazuya

Min, Kyung-Jin

Min, Kyung-San

Minafra, Luigi

Minami, Masaaki

Minami, Yosuke

Minamisawa, Susumu

Minarovits, Janos

Mindt, Thomas

Mine, Akira

Mine, Yuichi

Mineev, Konstantin

Mineo, Placido

Miners, Scott

Minervini, Crescenzio Francesco

Mingorance, Jesús

Minguet, Eugenio G.

Minh, David D. L.

Minh, Truongngoc

Minic, Zeljka

Minic, Zoran

Miniero, Daniela Valeria

Miniewicz, Andrzej

Minini, Mirko

Mino, Masanobu

Minocha, Subhash C.

Minorics, Renáta

Mintz, Cyrus David

Minucci, Sergio

Minutolo, Antonella

Mio, Catia

Miosge, Nicolai

Miotto, Benoit

Miquerol, Lucile

Mir, Hina

MIR, MOHAMMED A.

Mir, Tahir Maqbool

Miragliotta, Vincenzo

Miragoli, Michele

Miralles, Manuel G.

Miranda, Adelaide

Miranda, Enrique

Miranda, Hugo
Miranda, João M.

Mircioiu, Constantin

Miretti, Silvia

Mirkhani, Seyyed Alireza

Miro, Jordi

Mironov, Alexander

Mirossay, Ladislav

Mirshahi, Massoud

Mirzayans, Razmik

Mirzoev, Timur M.

Misasi, Roberta

Misawa, Eriko

Misciagna, Giovanni

Miserey-Lenkei, Stéphanie

Mishima, Hiroyuki

Mishima, Masaki

Mishin, Alexander S.

Mishra, Abheepsa

Mishra, Ajay Kumar

Mishra, Awdhesh

Mishra, Meerambika

Mishra, Nagendra N.

Mishra, Priyanka

Mishra, Rosalin

Mishra, Santosh K.

Mishto, Michele

Misicka, Aleksandra

Misiolek, Maciej

Miskiewicz, Piotr

Miskulin, Maja

Mistrik, Martin

Mistriotis, Panagiotis

Misu, Tatsuro

Misztalewska-Turkowicz, Iwona

Mita, Koji

Mitchell, Cassie S.

Mitchell, Michael J.

Mitchell, Murray

Mithoefer, Axel

Mitjans Arnal, Montserrat

Mitkin, Nikita A.

Mitola, Stefania

Mitoma, Hiroshi

Mitra, Joy

Mitrofanis, John

Mitsuhashi, Wataru

Mitsui, Toshiaki

Mitsuru, Okuwaki

Mitsuya, Shiro

Mitsuyama, Susumu

Mittal, Sandeep 
Mittnacht, Sibylle

Mitura, Katarzyna

Miura, Natsuko

Miura, Takashi

Miyado, Kenji

Miyagaki, Tomomitsu

Miyahara, Taira

Miyake, Masateru

Miyamoto, Koji

Miyamoto, Yohei

Miyashita, Shuhei

Miyata, Yoshihiko

Miyazaki, Motoyasu

Miyazaki, Teruo

Miyazaki, Tetsuro

Miyazaki, Toshiki

Miyoshi, Daisuke

Mizia-Stec, Katarzyna

Mizobata, Tomohiro

Mizuguchi, Mariko

Mizui, Toshiyuki

Mizuno, Hideaki

Mizuno, Tomohiro

Mizuo, Keisuke

Mizzotti, Chiara

Mkrtchian, Souren

Mladěnka, Přemysl

Mladenov, Emil

Mladenova, Irena

Mlaga, Kodjovi D.

Mlera, Luwanika

Mlinarić, Selma

Mlostoń, Grzegorz

Mlynarczyk, Dariusz

Mo, Chenglin

Mo, Fei

Mo, Yiqun

Mo, Youngjun

Moar, William

Moawad, Adel

Mobbili, Giovanna

Mobed-Miremadi, Maryam

Mochida, Sumiko

Mochizuki, Kazufumi

Moda, Fabio

Modahl, Cassandra

Modenutti, Carlos

Modesti, Alessandra

Modrowski, Dominique

Mogi, Masaki

Mogielnicki, Andrzej
Möglich, Andreas

Moha Ou Maati, Hamid

Mohamed, Azza H.

Mohamed, Heba

Mohamed, Junaith S.

Mohamed, Salah A.

Mohamed, Tamer

Mohamed, Tarek Magdy

Mohammad, Aliyu

Mohammed, Altaf

Mohammed, Yousuf

Mohan, Ganesh Babu Malli

Mohanta, Tapan Kumar

Mohanty, Smita

Mohanty, Vakul

Mohd Hanif, Zulfakar

Möhlmann, Torsten

Mohr, Susanne

Mohsen, Attayeb

Mohsin, Sadia

Moin, Mazahar

Moir, Anne J.G

Moiseev, Roman V.

Moiseyev, Gennadiy

Mojzych, Mariusz

Mok, Tze

Mokra, Daniela

Mokry, Jaroslav

Moktefi, Anissa

Mold, Matthew J.

Moldogazieva, N.T.

Molenaar, Remco J.

Molesini, Barbara

Molgó, Jordi

Molina, Cristina E.

Molina-Cerrillo, Javier

Molinari, Sergio

Molinari, Susanna

Molinier, Jean

Moll, Guido

Møller, Lisbeth Birk

Møller, Thor Christian

Molmenti, Ernesto P.

Molnár, Elek

Mołoń, Mateusz

Moloney, Gerard M.

Moltzau, Lise Román

Molyneux, Karen

Momekov, Gregori

Momeny, Majid

Monaco, Giovanni 
Monaghan, Daniel

Monari, Antonio

Monasky, Michelle

Moncharmont, Bruno

Monchatre-Leroy, Elodie

Mondal, Goutam

Monfrini, Marianna

Mong, Mei-Chin

Monget, Philippe

Mongin, Alexander A.

Monickaraj, Finny

Monjarás Feria, Julia

Monje, Paula V.

Monné, Magnus

Monokrousos, Nikolaos

Monroe, Glen R.

Monroy, Fernando

Monsalve, Maria

Monsarrat, Paul

Montag, Dirk

Montag, Judith

Montagne, Kevin

Montague, Samantha

Montalvo-Ortiz, Janitza L.

Montalvo-Rodríguez, Rafael

Montanari, Elita

Montanaro, Lorenzo

Montarolo, Francesca

Monte, Isabel

Montecucco, Fabrizio

Monteil, Christelle

Monteiro, Filipa

Monteiro, Mario

Monteiro, Sílvia

Monteleone, Ivan

Montemiglio, Linda

Montemurro, Cinzia

Montemurro, Nicola

Montenegro, Lucia

Montero, Olimpio

Montero-Melendez, Trinidad

Montes Resano, Marta

Montes, Raquel

Montesano, Domenico

Montesano, Vincenzo

Montesarchio, Daniela

Montesinos, Laura

Monti, Barbara

Montiel-Duarte, Cristina

Montiel-Smith, Sara

Montoliu-Gaya, Laia
Montoro, Miguel

Montrucchio, Giuseppe

Monville, Christelle

Monza, Laura

Moo, Eng Kuan

Moody, Suzy

Mooers, Blaine

Mookerjee, Shona

Moolhuijzen, Paula M.

Moon, Changjong

Moon, Il Soo

Moon, Jong-Seok

Moon, Minho

Moon, Seung Myung

Moon, Sung-Kwon

Moonen, Carolyn G.J.

Moore, Catrin

Moore, David

Moore, Geromy George

Moore, Matthew D.

Moore, Stuart

Moore, Thomas F

Moore-Carrasco, Rodrigo

Moorehead, Roger

Moosmann, Bernd

Morabito, Alessandro

Morabito, Antonino

Moraczewska, Johanna

Moradian-Oldak, Janet

Morais, Mauricio

Morais, Victor

Morales Suárez-Varela, María M.

Morales, Julio

Morales, Paula

Morales, Serafin

Morales, Víctor Abad

Morales-González, José Antonio

Moral-García, José Enrique

Mora-Montes, Hector

Moran, Jose

Moran, Oscar

Morán, Paloma

Morandi, Luca

Morante, Juan José Hernández

Moravcikova, Jana

Mordalski, Stefan

Moreira, Ana Carolina

Moreira, André Ferreira

Moreira, Daniel Carneiro

Moreira, Irina S.

Morel, Bertrand 
Morel, Jean-Luc

Morelli, Eugenio

Morelli, Maria Beatrice

Morello, Silvana

Moreno Amador, María Lourdes

Moreno, Adrian

Moreno, Jordi

Moreno-Gonzalez, Ines

Moreno-Loshuertos, Raquel

Moreno-Piraján, Juan Carlos

Moreno-Villanueva, Maria

Moretto, Alessandro

Morffy, Nicholas

Morgan, Ethan L.

Morgan, Philip

Morgan, Rhianna K.

Morgante, Giuseppe

Mori, Kiyoshi

Mori, Masaki

Mori, Shigeki

Moricoli, Diego

Moriguchi, Takaya

Morikawa, Satoru

Morikawa, Toshio

Morikawa, Yuko

Morimoto, Kinjiro

Morimoto, Tatsuya

Morimoto, Yasuo

Morimura, Shigeru

Morini, Luca

Morini, Martina

Morino, Katsutaro

Morison, Ian

Morita, Kenta

Morita, Manabu

Morita, Mitsuhiro

Morita, Naoki

Morita, Shigeto

Moritake, Takashi

Moritz, Michał

Moriya, Makoto

Moriyama, Shtjnsuke

Moriyama, Yasuko

Morland, Cecilie

Morleo, Manuela

Morohashi, Kengo

Moros, María

Morouço, Pedro

Morozov, Oleg G.

Morozov, Vitaly A.

Morozov, Vladimir N.
Morozova-Roche, Ludmilla

Morra, Marco

Morreau, Hans

Morrice, Nicola

Morrione, Andrea

Morris, Andrew

Morris, Jill K.

Morris, Keith

Morris, Paul Francis

Morrison, Douglas

Morroni, Fabiana

Morrow, John P.

Morsczeck, Christian

Morselli, Marco

Mortara, Lorenzo

Morten, Karl J.

Mortensen, Luke J.

Mortensen, Martin S.

Mosbah, Ismail Ben

Mosca, Luciana

Moschini, Roberta

Moschou, Panagiotis

Moschovi, Maria

Moscucci, Federica

Mosgöller, Wilhelm

Moshiri, Ala

Moshiri, Arfa

Mosienko, Valentina

Moskalev, Alexey A.

Moškon, Miha

Mosqueira, Diogo

Mosqueira, Matias

Moss, Anthony G.

Moss, Don

Moss, Walter

Mossel, Eric

Mota, Manuel

Motakis, Efthymios

Motlík, Jan

Motloch, Lukas J

Motloch, Lukas J.

Motohashi, Ken

Motose, Hiroyasu

Motta, Irene

Mottes, Monica

Mou, Haiwei

Mou, Yongchao

Moudgil, Kamal

Moujir, Laila M.

Moukhles, Hakima

Moulin, Alexandre 
Mourão, Carlos Fernando

Mousa, Shaaban A.

Mousa, Shaker

Mousley, Carl

Moustaïd-Moussa, Naïma

Moutinho, Miguel

Mouzaki, Athanasia

Mouzeyar, Saïd

Moy, Jamie

Moyes, David

Mozgova, Iva

Mozos, Ioana

Mracek, Tomas

Mravec, Jozef

Mróz, Waldemar

Msaouel, Pavlos

$\mathrm{Mu}$, Hai-Jin

$\mathrm{Mu}$, Ping

Muccilli, Vera

Muceniece, Ruta

Mucignat, Carla

Mück-Lichtenfeld, Christian

Mucsi, Zoltán

Mudgil, Poonam

Mueller, Wolf C.

Mueller-Roeber, Bernd

Muff, Roman

Mugisho, Odunayo

Mühle, Christiane

Mühlfeld, Christian

Muhovski, Yordan

Mu-Hsin, Chang

Muhvic Urek, Miranda

Mujtaba, Shiraz

Mukai, Tomoyuki

Mukherjee, Amitava

Mukherjee, Dwaipayan

Mukherjee, Nabanita

Mukherjee, Rajib

Mukherjee, Sarbajit

Mukherjee, Somnath

Mukherjee, Sudip

Mukhtar, Karolina

Mukwaya, Anthony

Mulcahy, Ben

Mulder, Callista

Mulet, Jose M.

Mulherkar, Shalaka

Mulholland, David

Muller Kratz, Jadel

Müller, Christoph W.
Müller, Hans-Arno J.

Muller, Laurent

Muller, Mandy

Muller, Marc

Muller, Miryam

Müller, Thomas Ernst

Müller-Deubert, Sigrid

Müller-Vahl, Kirsten R.

Mulligan, Vikram K.

Mullin, James M.

Multhoff, Gabriele

Mulvey, Matthew

Mulvihill, Erin

Munafo, John

Munarin, Fabiola

Munaron, Luca

Muniraj, Nethaji

Muniswamy, Madesh

Munk, Carsten

Muñoz, Alfonso

Muñoz, Francisco José

Muñoz, Victor

Muñoz-Castañeda, Juan R.

Muñoz-Chápuli, Ramón

Muñoz-Descalzo, Silvia

Muñoz-Félix, José M.

Muñoz-Torrero, Diego

Munro, David

Muntané, Gerard

Muntane, Jordi

Muntean, Alexandrina

Munye, Mustafa M.

Münzer, Patrick

Mupo, Annalisa

Murab, Sumit

Muraca, Maurizio

Murach, Kevin

Murai, Koji

Muraille, Eric

Murakami, Kohei

Murakami, Yasufumi

Murakoshi, Nobuyuki

Murale, Dhiraj P.

Muraleva, Natalia A.

Muranaka, Atsuya

Murani, Eduard

Muranov, Konstantin

Murase, Daiki

Murase, Sachiko

Murashita, Koji

Murata, Hitoshi 
Murata, Jun

Murata, Soichiro

Murata, Takayuki

Muratani, Masafumi

Muratori, Monica

Muratov, Eugene N

Muravnik, Lyudmila E.

Murawska-Ciałowicz, Eugenia

Murciano-Calles, Javier

Murdica, Valentina

Muresan, Mihai Stefan

Murias, Marek

Murín, Radoslav

Murkovic, Michael

Muroya, Susumu

Muroya, Yoshikazu

Murphy, Andrew

Murphy, Ciara M.

Murphy, Denis

Murphy, Eain

Murphy, Ronan

Murphy-Ullrich, Joanne E.

Murray Stewart, Tracy

Muruganandan, Shanmugam

Musacchia, Francesco

Musalgaonkar, Sharmishtha

Musani, Vesna

Musazzi, Laura

Musch, Mark

Muschiol, Jan

Muschol, Martin

Musil, Linda S.

Musilek, Kamil

Musk, Gabrielle C.

Mußbacher, Marion

Musso, Natale

Musson, David S.

Mustachio, Lisa

Mustafa, Ghazala

Musti, Anna Maria

Musulen, Eva

Musumeci, Giuseppe

Muszbek, László

Muszyńska, Ewa

Muszyński, Siemowit

Muthamilarasan, Mehanathan

Muthu Karuppan, Mohan Kumar

Muthukumar, Alagar R.

Muthukumar, Kannan

Muthuramu, Ilayaraja

Muthusamy, Nagendran
Mutis, Tuna

Mutoh, Tatsuro

Mutsaers, Steven E.

Mutti, Luciano

Muyldermans, Serge

Muz, Barbara

Muziol, Tadeusz Mikolaj

Muzykantov, Vladimir R.

Mylonas, Ioannis

Mylonis, Ilias

Mymryk, Joe

Myrianthopoulos, Vassilios

Mysiukiewicz, Olga

Mysliveček, Jaromír

Myśliwiec, Hanna

Mysliwiec, Tami

Mytych, Jennifer

Myung, Heejoon

$\mathrm{Na}$, Dokyun

$\mathrm{Na}$, Dong Gyu

$\mathrm{Na}$, Dong Hee

$\mathrm{Na}$, Sungsoo

Nabissi, Massimo

Naccarato, Marcello

Nacry, Philippe

Nadai, Matteo

Nadakuduti, Satya Swathi

Nadeau, Jay L.

Nadeem, Muhammad

Nademanee, Koonlawee

Naderi, Nassim

Nadiminty, Nagalakshmi

Naegeli, Hanspeter

Naftalin, Richard

Nag, Okhil K.

Nagahage, Isura

Nagai, Noriaki

Nagajyothi, Jyothi F

Nagano, Seiichi

Nagaoka, Isao

Nagaraj, Ram H.

Nagaraj, Vini

Nagarajan, Balaji

Nagarajan, Prabakaran

Nagarajan, Uma M.

Nagasaka, Kazunori

Nagasawa, Kazue

Nagashima, Yukihiro

Nagata, Eiichiro

Nagata, Naoto

Nagata, Shinji 
Nagata, Takashi

Nagatoishi, Satoru

Nagatomo, Yuji

Naghibalhossaini, Fakhraddin

Nagy, Bálint

Nagy, Béla

Nagy, Corina

Nagy, Elod

Nagy, Endre V.

Nagy, Istvan

Nagy, Tamás

Nahalkova, Jarmila

Nahi, Hareth

Nair, Sunila G.

Naito, Tateaki

Naito, Toshio

Najar, Amer

Najdenski, Hristo

Najera, Francisco

Naka, Hiroaki

Nakabayashi, Kazumi

Nakagawa, Hiroshi

Nakagawa, Naoki

Nakagawa, Takuro

Nakagawa, Tetsuya

Nakagomi, Takayuki

Nakahama, Ken-ichi

Nakahara, Tsutomu

Nakahata, Shingo

Nakajima, Akira

Nakamaru-Ogiso, Eiko

Nakamichi, Norihito

Nakamura, Kazufumi

Nakamura, Keigo

Nakamura, Kohei

Nakamura, Kyohei

Nakamura, Masanao

Nakamura, Masato

Nakamura, Motoki

Nakamura, Nobuhiro

Nakamura, Noriko

Nakamura, Shin-ichi

Nakamura, Tomoki

Nakamura, Yoki

Nakamura, Yoshiyuki

Nakano, Michiharu

Nakano, Shuichi

Nakano, Takanari

Nakano, Tamaki

Nakano, Toshiaki

Nakao, Reiko
Nakase, Hiroshi

Nakase, Ikuhiko

Nakashima, Kazuo

Nakata, Miyuki

Nakatani, Yoshihiko

Nakatsu, Kanji

Nakatsuka, Akira

Nakatsukasa, Kunio

Nakayama, Hironao

Nakayama, Hokuto

Nakayama, Jun

Nakayama, Masafumi

Nakayama, Masayoshi

Nakayama, Naomi

Nakayasu, Ernesto

Nakazawa, Mitsuru

Nakchbandi, Inaam

Nakhjavani, Maryam

Nale, Janet

Nalli, Cecilia

Nalvarte, Ivan

Nam, Jin-Wu

Nam, Ki Hyun

Nam, Kung-Woo

Nam, Seung Yun

Nam, Young-Jae

Namasivayam, Vigneshwaran

Nambara, Eiji

Namgaladze, Dmitry

Namihira, Masakazu

Nanba, Eiji

Nand, Ashveen

Nanda, Satyabrata

Nanda, Sitansu Sekhar

Nandi, Deipanjan

Nandi, Shyam

Nandi, Somen

Nanduri, Ravikanth

Nanno, Masanobu

Nanoff, Christian

Nanou, Afroditi

Naoe, Yoshinori

Naoumkina, Marina

Naponelli, Valeria

Nappi, Lucia

Naqash, Abdul Rafeh

Naranjo, Tomás

Narayan, Edward

Narayan, Mahesh

Narayanan, Ganesh

Nardecchia, Stefania 
Nardelli, Carmela

Nardi, Francesco

Nardi, James B.

Nardi, Serenella

Nardini, Marco

Narendra, Derek

Narita, Shintaro

Narra, Hema Prasad

Naruishi, Koji

Narute, Purushottam

Narváez, Manuel

Narwani, Tarun JaiRaj

Nascimento, Weslania Viviane

Nash, Kevin

Nashimoto, Yuji

Nashine, Sonali

Nasi, Milena

Nasim, Muhammad Jawad

Nasim, Talat

Nasim, Zeeshan

Nassa, Giovanni

Nassar, Zeyad D.

Nässel, Dick R.

Nasser, Mohd Wasim

Nassini, Romina

Nassir, Fatiha

Nassisi, Marco

Nastasa, Cristina

Nastasi, Claudia

Nasti, Tahseen H

Nastoupil, Loretta J

Nat, Irina Roxana

Natale, David

Natale, Gianfranco

Natarajan, Purushothaman

Natarajan, Sivaraman

Natarajan, Viswanathan

Natarelli, Lucia

Natesan, Shanmugasundaram

Nath, Audrey

Nath, Niharika

Nath, Shubhankar

Nathanailides, Cosmas

Nathanson, Lubov

Natile, Giovanni

Natoli, Silvia

Naujok, Ortwin

Nauli, Surya

Nault, Rance

Naumov, Anton V.

Naumovski, Nenad
Naumowicz, Monika

Nava, Sara

Navarra, Michele

Navarrete-Vazquez, Gabriel

Navarro, Elisa

Navarro, Estanis

Navarro, Rodrigo

Navarro-Guillén, Carmen

Navarro-López, Juan D.

Navas, Alfonso

Naveed Yousaf, Muhammad

Naves, Thomas

Naviglio, Samuele

Navizet, Isabelle

Navone, Stefania

Nawade, Bhagwat

Nawaz, Fahim

Nawirska-Olszańska, Agnieszka

Nawrot, Barbara

Nawrot, Robert

Nayak, Manasa K.

Nayak, Nihar

Naylor, Kari

Nazar, Ross N.

Nazare, Marc

Nazarov, Alexey

Nazaruk, Ewa

Nazimek, Katarzyna

Nazio, Francesca

Nazzaro, Gianluca

Ndika, Joseph

Neagu, Adrian

Nechepurenko, Ivan V.

Neda, Ion

Neelakantan, Prasanna

Neelam, Srujana

Neerman-Arbez, Marguerite

Nees, Matthias

Neffe, Axel T.

Nefzger, Christian

Neginskaya, Maria A.

Negrea, Petru

Nehela, Yasser

Neidlinger-Wilke, Cornelia

Neira, José L.

Nekhai, Sergei

Nelson, Amanda M.

Nelson, Andrew

Nelson, Dorothy A

Nelson, Fred R.T.

Nelson, Randy 
Nemec, Ivan

Nemery, Benoit

Nemes, Katalin

Nemeș, Roxana Maria

Németh, András György

Nemeth, Zoltan H

Nemirovskaya, Tatiana L.

Nemoto, Kiyomitsu

Nemoto, Wataru

Nenna, Antonio

Nenoi, Mitsuru

Nenov, Miroslav N.

Neo, Ana G.

Neophytou, Christiana M.

Nepal, Mahesh Raj

Neri, Giovanni

Neri, Matteo

Neri, Simona

Nesan, Dinushan

Nesbitt, Warwick S.

Nesmelov, Yuri E.

Nestares Pleguezuelo, Maria Teresa

Nesterov-Mueller, Alexander

Nestor, Casey

Nestrasil, Igor

Nethi, Susheel Kumar

Nettersheim, Daniel

Neubauer, Damian

Neubert, Reinhard

Neufeld, Howard

Neufeldt, Christopher John

Neufurth, Meik

Neugebauer, Dorota

Neuhaus, Jochen

Neuhauss, Stephan

Neuhuber, Winfried L.

Neumann, Brent

Neumann, Donna

Neumann, Jake

Neunaber, Claudia

Neupane, Mahesh

Neupane, Yub Raj

Neureiter, Daniel

Nevárez-Moorillón, Guadalupe Virginia

Neves, João

Neves, Vera

Nevi, Lorenzo

Nevoral, Jan

Newmire, Daniel

Newsome, Timothy P.

Newton, Jason
Newton, Robert

Neyroud, Daria

Nezhat, Farr Reza

$\mathrm{Ng}$, Ho Leung

$\mathrm{Ng}$, Hooi Hooi

$\mathrm{Ng}$, Keng Wooi

$\mathrm{Ng}$, Pei Ying

$\mathrm{Ng}$, Ray

$\mathrm{Ng}$, Tzi Bun

$\mathrm{Ng}$, Wai-Lung

Ngezahayo, Anaclet

Nguyen, Chi Huu

Nguyen, COL Chi

Nguyen, Duy

Nguyen, Khoi Tan

Nguyen, Linh T.B

Nguyen, Long The

Nguyen, Minh Tho

Nguyen, Quynh

Nguyen, Thanh Hung

Nguyen, Trieu

Ngwa, Che Julius

Nianiou-Obeidat, Irini

Nicaise, Charles

Niccoli, Teresa

Niclos Gutiérrez, Juan

Nicolas, Cyril

Nicolas, Emmanuelle

Nicolás, Francisco J.

Nicolas, Gaël

Nicolazzo, Joseph

Nicoletti, Ferdinando

Nicoletti, Vincenzo

Nicolì, Francesca

Nicolini, Gabriella

Nicoll, Amanda J.

Nicoll, Rachel

Niculae, Dana

Niculescu, Loredan

Niczyporyk, Jowita Samanta

$\mathrm{Nie}, \mathrm{Yu}$

Niederberger, Ellen

Niederseer, David

Niederwieser, Dietger

Niehrs, Christof

Nielsen, Claus $\mathrm{H}$.

Nielsen, Morten

Nielsen, Thorbjørn Terndrup

Nielsen, Vance Girard

Niemann, Carsten

Niemczyk, Mariusz 
Nieminen, Taina $\mathrm{T}$.

Niemirowicz-Laskowska, Katarzyna

Nieoczym, Dorota

Niesor, Eric J.

Nieuwenhuizen, Niels J.

Nievergelt, Adrian Pascal

Nifantiev, Nikolay

Nifli, Artemissia-Phoebe

Nighot, Prashant

Nijnik, Anastasia

Nikishin, Denis

Nikitin, Dmitri V.

Nikitovic, Dragana

Nikodemova, Maria

Nikolaev, Anton

Nikolaidis, Nikolas

Nikolaivits, Efstratios

Nikolakaki, Eleni

Nikolakakis, Ioannis

Nikolakopoulou, Angeliki M.

Nikolay, Rainer

Nikolic, Dejan

Nikolich, Mikeljon

Nikoloudakis, Nikolaos

Nikolova, Irina

Nikonenko, Victor

Nilsen-Hamilton, Marit

Nilsson, Maria

Nina, Brutch

Ninni, Sandro

Nirala, Niraj K.

Nishi, Mayumi

Nishi, Ryotaro

Nishibori, Masahiro

Nishida, Emi

Nishida, Haruto

Nishida, Kensei

Nishida, Sho

Nishida, Shozo

Nishiguchi, Masamichi

Nishihara, Hidenori

Nishihara, Ryo

Nishikawa, Kiisa

Nishikawa, Masaki

Nishikawa, Yuji

Nishimura, Takeshi

Nishiyama, Akiko

Nishiyama, Akira

Nishiyama, Yoshitaka

Nishiyama, Yusuke

Nishizawa, Yoko
Nishizuka, Makoto

Nislow, Corey

Nisoli, Enzo

Nissen, Lorenzo

Nissen, Peter H

Nistri, Andrea

Nitti, Maria Paola

Nitulescu, George Mihai

Nitulescu, Georgiana

Nitulescu, Mihai

Niu, Dau-Ming

Niu, Fang

Niu, Hengyao

Niu, Tianhua

Nixon, Daniel W.

Niyazov, Dmitriy M.

Njoku, Dolores B.

Nobile, Stefano

Nobili, Stefania

Nobis, Max

Nóbrega, Clévio

Nóbrega-Pereira, Sandrina

Nobs, Samuel Philip

Nocentini, Alessio

Nocentini, Giuseppe

Nociari, Marcelo

Nofer, Jerzy-Roch

Nogales Gadea, Gisela

Nogales, Aitor

Nogales-Gadea, Gisela

Noghero, Alessio

Noguchi, Constance Tom

Noguchi, Satoru

Noguchi, Takuya

Nogueira, Paulo Jorge

Noguera, Nélida Inés

Nogues, Isabel

Nohara, Kazunari

Nohata, Nijiro

Nohe, Anja

Nolan, John

Nolan, Tania

Nollert, Matthias U.

Noll-Hussong, Michael

Nolte, Ingo

Nomura, Seitaro

Nonaka, Taichiro

Nonami, Atsushi

Nonell, Santi

Nonomura, Ken-ichi

Noonepalle, Satish 
Noorani, Imran

Noordam, Raymond

Norcic, Gregor

Nordén, Rickard

Noren Hooten, Nicole

Norgren, Lars

Noriega, Fernando G.

Norinder, Ulf

Noritaka, Yamaguchi

Norman, Kenneth

Norman, Trevor

Normark, Benjamin

Norton, Kerri-Ann

Norton, Michael

Norval, Mary

Nosch, Daniela Sonja

Nosoudi, Nasim

Notaras, Michael J.

Notarbartolo, Monica

Notario-Pérez, Fernando

Notarstefano, Valentina

Nottegar, Alessia

Noubissi, Felicite Kamdem

Noureddine, Achraf

Nouvian, Regis

Novak Kujundžić, Renata

Novak, Iona

Novak, Sanja

Novick, Daniela

Novick, Richard P.

Novikov, Alexander S.

Novikov, Arthur I.

Novinec, Marko

Novío, Silvia

Novo, Luís

Novoplansky, Ariel

Novo-Veleiro, Ignacio

Nowacka, Maria

Nowaczyk, Alicja

Nowak, Adriana

Nowak, Romana A.

Nowicka, Grażyna

Nowicki, Marcin

Nowicki, Michał

Nowicki, Roman

Nowotny, Marcin

Nozaki, Masami

Nozumi, Motohiro

Ntougias, Spyridon

Nuber, Silke
Nuc, Katarzyna

Nuc, Przemysław

Nucci, Nathaniel

Nucera, Eleonora

Nudler, Evgeny

Nuesch, Jurg

Numakawa, Tadahiro

Nunes, Fernando

Nunes, Francis Morais Franco

Nunez De Cáceres González, Francisco

Federico

Nunziata, Angelina

Nunzio, Vicario

Nussbaumer, Thomas

Nuță, Diana Camelia

Nuzziello, Nicoletta

Nuzzo, Domenico

Nwaiwu, Ogueri

Nwosu, Zeribe Chike

Nylander, Karin

Nypelo, Tiina

Nyström, Alexander

Nzau Matondo, Billy

O'Callaghan, David

O'Connell, Thomas

O'Connor, Christine

O'Day, Danton

O'Dell, William Brad

O'Halloran, Kenneth

O'Keeffe-Ahern, Jonathan

O'Leary, Brendan M.

O'Leary, Seán

O'Leary, Seonadh

O'Neal, Hollis

O'Neal, Matthew E.

O'Reilly, Kally C.

O'Reilly, Steven

O'Rourke, Jamie

O'Sullivan, Jeremy A.

Oakhill, Jon

Obana, Akira

Obara, Yutaro

Obata, Yasuko

Obeng, Samuel

Oberemok, Volodymyr V.

Obermüller, Nicholas

Obeso, Ana

Obialo, Chamberlain I.

Obinata, Daisuke

Obrador, Elena

Obri, Arnaud 
Obuchowski, Michal

Obukhov, Alexander G.

Ochatt, Sergio

Ochayon, David E.

Ochi, Shinichiro

Ochiai, Daigo

Ochoa, Theresa

Ochoa-Reparaz, Javier

Ochs, Raymond S.

Oczkowicz, Maria

Odagi, Minami

Oddi, Sergio

Odenthal, Margarete

Odierna, Gaetano

Odintsova, Tatyiana I.

Oechslin, Frank

Ofir, Rivka

Ogai, Kazuhiro

Ogami, Koichi

Ogasawara, Toru

Ogata, Yoshiyuki

Ogawa, Akiko

Ogawa, Hidehiko

Ogawa, Youichi

Ogbureke, Kalu UE

Oger, Philippe M.

Ogino, Shuji

Ogneva, Irina

Ognibene, Marzia

Ogórek, Rafał

Ögren, Sven O.

Ogura, Takehiko

Oh, Byungchul

Oh, Jun

Oh, Jun-Seok

Oh, Man-ho

$\mathrm{Oh}$, Min Kyu

Oh, Seikwan

Oh, Soo Young

Oh, Yohan

Ohashi, Manabu

Ohashi, Riuko

Ohko, Kentaro

Ohkubo, Hirotsugu

Ohlmann, Andreas V.

Ohlsson, Sophie M.

Ohmido, Nobuko

Ohmiya, Yoshhiro

Ohneda, Kinuko

Ohnishi, Shiho

Ohno, Jun
Oho, Takahiko

Öhrfelt, Annika

Ohta, Yuko

Ohtsuka, Akira

Ohtsuka, Masayuki

Ohtsuki, Gen

Ohtsuki, Takashi

Ohya, Susumu

Ohya, Yoshikazu

Oikawa, Tsunekazu

Oiso, Naoki

Ojha, Rani

Oka, Yuji

Okada, Hiroshi

Okada, Motohiro

Okada, Tomoyo

Okae, Hiroaki

Okamoto, Curtis

Okamoto, Mamoru

Okamoto, Masami

Okamoto, Takayuki

Okamura, Yu

Okazaki, Satoshi

Okazaki, Yozo

Okazawa, Atsushi

Okba, Nisreen M.A.

Okegawa, Takatsugu

Okita, Thomas W.

Okleštková, Jana

Oklinski, Michal Krystian Egelund

Okochi, Mina

Okoń, Sylwia

Okuda, Nagako

Okuda-Ashitaka, Emiko

Okumura, Akihisa

Okumura, Masaki

Okumura, Nobuaki

Okumura, Nobuo

Oláh, Julianna

Olar, Rodica

Olczyk, Krystyna

Olczyk, Paweł

Oldfield, Eric

Oleksiewicz, Urszula

Oliva, Francesco

Oliva, María Ángela

Oliveira, Ana M.M.

Oliveira, Mariana Bleker De

Oliveira, Mariana Braga

Oliveira, Paula A.

Oliveira, Rui S. 
Olivier, Jean Luc

Oliviera, Pedro

Olkkonen, Vesa M.

Öllinger, Robert

Olmo, Francisco

Olnes, Matthew J.

Olova, Nelly

Olsen, Christopher M.

Olson, Matthew

Olson-Manning, Carrie

Olsson, Martin

Olszewska, Marta

Olszewska, Monika A

Oltolina, Francesca

Oltra, Elisa

Omar, Ahmad A.

Omori, Yasufumi

Omote, Hiroshi

Omura, Junichi

Ondřej, Vladan

Onel, Buket

Onimaru, Hiroshi

Onkokesung, Nawaporn

Ono, Masanori

Ono, Noriaki

Ono, Takashi

Ono, Yusuke

Onomoto, Koji

Onori, Paolo

Onukwufor, John O.

Onyango, Isaac

Onyeagucha, Benjamin Chidi

Oorschot, Dorothy

Opaliński, Łukasz

Opiela, Jolanta

Opländer, Christian

Opocher, Enrico

Oppert, Brenda

Oralová, Veronika

Oramas-Royo, Sandra

Orban, John

Ördög, Attila

Orecchioni, Marco

Orefice, Nicola Salvatore

Orekhov, Alexander N.

Orellana, Esteban

Orelle, Cédric

Oren, Yoram

Orenes-Piñero, Esteban

Orengo, Dorcas

Oresnik, Ivan
Orf, Isabel

Orian, Laura

Orii, Kenji E.

Orive, Alejandro Gonzalez

Orkusz, Agnieszka

Orlandi, Augusto

Orlandi, Viviana Teresa

Orlandini, Maurizio

Orlando, Giustino

Orloff, Marlana

Orłowski, Marek

Ornoy, Asher

Orosco, Pablo

Orrú, Christina

Orsini, Joe

Orso, Evelyn

Orsolic, Nada

Orsucci, Daniele

Ortan, Alina

Ortega, Angel L.

Ortega, Eduardo

Ortega, Francisco José

Ortega, Miguel Angel

Ortega-Gutierrez, Silvia

Ortega-Villaizan, Maria Del Mar

Ortín-Martínez, Arturo

Ortiz, J. Bryce

Ortiz, Jorge

Ortiz, Maria J.

Ortiz-Masiá, Dolores

Ortolani, Fulvia

Ortolano, Saida

Orton, Thomas J.

Orusa, Riccardo

Orynbayeva, Zulfiya

Orzan, Francesca

Orzechowski, Arkadiusz

Orzelska-Górka, Jolanta

Osborn, Mark

Osborn, Stephanie L.

Osella, Silvio

Osellame, Laura D.

Oshi, Masanori

Oshima, Shunji

Oshima, Yusuke

Osipova, Zinaida

Osmanagic-Myers, Selma

Osório, Daniel

Osorio, Elvia Janeth

Osornio, Álvaro

Ostaszewski, Ryszard 
Oster, Henrik

Oster, Michael

Ostersetzer-Biran, Oren

Ostos Marcos, Francisco José

Ostrowski, Daniela

Ostrowski, Maciej

Ostróżka-Cieślik, Aneta

Ostuni, Mariano

Oswald, Stefan

Osyczka, Anna Maria

Oszako, Tomasz

Otani, Masahiro

Oteri, Giacomo

Otero-Vinas, Marta

Othumpangat, Sreekumar

Otręba, Michał

Otsuguro, Ken-ichi

Otsuka, Isao

Otsuka, Yuzuru

Otsuki, Takemi

Ottmann, Christian

Ottolenghi, Sara

Otulak-Kozieł, Katarzyna

Ötvös, Krisztina

Otzen, Daniel

Ou, Hsiu-Chung

Ou, Shujun

Ouchi, Takehito

Ougham, Helen

Ouro, Alberto

Ouweland, Jody M. W. Van Den

Ouyang, Xinhao

Ovádi, Judit

Ovecka, Miroslav

Overgard, Aina-Cathrine

Ovsepian, Saak V

Ovsyannikov, A. S.

Owczarczyk-Saczonek, Agnieszka

Owen, Carolyn

Owen, Robert

Owen, Shawn

Oxford, Julia

Oyarzabal, Alfonso

Oyoshi, Takanori

Oza, Javin

Ozaki, Iwata

Özdinler, P. Hande

Ozeki, Yasuhiro

Ozga, Andrew

Ozretić, Petar

Pabbidi, Reddy M
Pabla, Navjot

Pabst, Andreas Max

Pacal, Lukas

Pacella, Elena

Pacelli, Consiglia

Pachulska-Wieczorek, Katarzyna

Paci, Maurizio

Pacia, Marta Z.

Pacifici, Luciano

Pacifico, Francesco M.

Pacifico, Severina

Paciolla, Costantino

Pacios, Luis F.

Packiam, Vignesh T.

Paço, Ana

Pacquelet, Anne

Pacurari, Maricica

Paczesny, Jan

Padan, Etana

Padanilam, Babu

Padera, Timothy

Padhi, Bhaja Krushna

Padhi, Soumesh

Padial-Molina, Miguel

Padro, Teresa

Paduch, Roman

Padula, Matthew P.

Pădureanu, Vlad

Paduszynska, Malgorzata

Pae, Eung-Kwon

Pafili, Kalliopi

Pagán, Israel

Pagani, Stefania

Pagano, Alessandra

Pagano, Bruno

Pagano, Cinzia

Pagano, Duilio

Pagano, Luca

Pagano, Mario

Pagano, Stefano

Page, Richard C.

Pageau, Karine

Pagella, Pierfrancesco

Pagenstecher, Axel

Pagliai, Fernando A.

Pagliarani, Chiara

Pagliardini, Luca

Pagnotta, Mario A.

Pagonopoulou, Olga

Pagotto, Sara

Pagter, Majken 
Pahwa, Roma

Paidi, Santosh Kumar

Paik, Taejong

Paine, Ananta

Paino, Francesca

Pais, Maria Salomé

Pais, Pedro

Pajak, Beata

Pajares, Marta

Pakdel, Farzad

Paknahad, Ali

Pal, Debjani

Pal, Krishnendu

Pál, Magda

Pal, Rumpa

Pal, Sangita

Palacios Calvo, Jose

Palacios, Isabel M.

Palacios, Javier

Palacios, José-Manuel

Paladini, Maria Serena

Paladino, Simona

Palage, Mariana

Palani, Chitra

Palaniappan, Balasubramanian

Palanisamy, Arulselvan

Pałasz, Artur

Palazzo, Barbara

Palazzo, Enza

Palese, Luigi Leonardo

Palestini, Paola

Palicka, Vladimir

Palini, Simone

Paliogiannis, Panagiotis

Pali-Schöll, Isabella

Palko-Labuz, Anna

Pall, Martin

Pallag, Annamária

Pallardó, Federico V.

Pallàs Lliberia, Mercè

Palle, Komaraiah

Pallotta, Isabella

Pallottini, Valentina

Palm, Gottfried J.

Palma, Angelina S.

Palma, José

Palma, Paulo Jorge

Palmer, Alan

Palmer, Michael

Palmer, Nathan A.

Palmer, Timothy Martin
Palmerini, Maria

Palmieri, Erika

Palmieri, Ferdinando

Palmieri, Giuseppe

Palmirotta, Raffaele

Palmisano, Alida

Palmour, Roberta

Palomba, Letizia

Palomo-Ríos, Elena

Palou, Mariona

Palsamy, Periyasamy

Palsdottir, Vilborg

Paluch, Andrew S.

Palumbo, Domenico

Palumbo, Giuseppe

Palumbo, Vincenzo Davide

Paluri, Ravi

Palus, Katarzyna

Paluszczak, Jarosław

Paluzzi, Jean-Paul V.

Pamarthy, Sahithi

Pamir, Nathalie

Pampaloni, Francesco

Pan, Chun-Hsu

Pan, Haihua

Pan, Hung-Chuan

Pan, Jiayi

Pan, Jing

Pan, Minggui

Pan, Ming-Kai

Pan, Tai-long

Pan, Tung-Ming

Pan, Xiaoyue

Pan, Yangang

Pan, Ying-xian

Pan, Youlian

Panara, Francesco

Panasiewicz, Grzegorz

Panatala, Radhakrishnan

Panchapakesan, Balaji

Panda, Amaresh C.

Panda, Kaushik

Panda, Santosh

Pandey, Dhananjay K.

Pandey, Pankaj

Pandey, Santosh

Pandiyan, Thangarasu

Pandolfi, Laura

Pandolfini, Tiziana

Pandya, Anshul A.

Panegyres, Peter K. 
Panek, Jaroslaw

Paneni, Francesco

Paneru, Bam

Panga, Jaipal

Pangestu, Mulyoto

Pangrazzi, Luca

Paniagua, Candelas

Pankonien, Ines

Pankotai, Tibor

Pankratova, Stanislava

Pannaccione, Anna

Panos, Anthony L.

Panos, Georgios D.

Panov, Konstantin

Panova-Noeva, Marina

Pant, Bishweshwar

Pantaleo, Antonella

Pantea Stoian, Anca

Panteris, Emmanuel

Pantham, Priyadarshini

Panuzzo, Cristina

Panwar, Priyanka

Panyutin, Igor G.

Panzarasa, Guido

Panzer, Simon

Pao, Ping-Chieh

Paolella, Gaetana

Paolini, Moreno

Paolo Vincenzo, Pedone

Papaccio, Federica

Papaccio, Gianpaolo

Papadaki, Chara

Papadimitriou, Dimitra N.

Papadopoulos, Dimitrios

Papageorgiou, Anastassios

Papageorgiou, Anastassios C.

Papageorgiou, George Z.

Papagerakis, Pétros

Papaioannou, Vasilios E

Papalois, Vassilios

Papamitsou, Theodora

Papanas, Nikolaos

Papanikolaou, Eleni

Paparella, Ashleigh S.

Papenbrock, Jutta

Papi, Piero

Papini, Alessio

Paplinska, Magdalena

Paplińska-Goryca, Magdalena

Papoutsoglou, Panagiotis

Papp, András
Papp, Béla

Papp, István

Pappas, Maria L.

Pappone, Carlo

Papp-Wallace, Krisztina M.

Paquette, Benoit

Par, Gabriella

Parajuli, Nirmala

Parajuli, Rajendra

Paranjape, Anurag

Parashar, Deepak

Paraskeva, Efrosyni

Paraskevas, George P.

Parchem, Ronald J.

Pardal, Ricardo

Pardini, Barbara

Pardo, Fatima

Parekh, Anant

Parent, Romain

Parente, Paola

Parenti, Ilaria

Parida, Sheetal

Parikesit, Arli Aditya

Parimon, Tanyalak

Parinandi, Narasimham

Paris, Debora

Paris, Francois

Parisi, Kathy

Park, Ah-Mee

Park, Byung-Wook

Park, Chan Ho

Park, Daeho

Park, Edwards A.

Park, Eujin

PARK, Eun Jeong

Park, Eun-Mi

Park, Gyungsoon

Park, Han-A

Park, Hyun-Woo

Park, Jae-Bong

Park, Jeewoong

Park, Ji-Won

Park, John

Park, Jong Kook

Park, Jong-Seok

Park, Joo-Cheol

Park, Joongkyu

Park, Jun-Beom

Park, Junseong

Park, Junsoo

Park, Junyoung O. 
Park, Kwang Hwan

Park, Ky Young

Park, Kyeongsoon

Park, Kyung-soon

Park, Kyu-Sang

Park, Myoung-Hwan

Park, Phil June

Park, Phun Bum

Park, Sang Won

Park, Sang-Wook

Park, See-Hyoung

Park, Shin-Hyung

Park, Sung-Joon

Park, Wansu

Park, Won Min

Park, Won Soon

Park, Yong-Doo

Parkar, Shanthi G.

Parker, Daniel M.

Parker, Edward

Parker, Lewan

Parker, Steven L.

Parkkila, Seppo

Parkkita, Seppo

Park-min, Kyung Hyun

Parks, Ruth

Parks, William C.

Parl, Fritz

Parlatescu, Ioanina

Parnell, Laura K.S.

Parnetti, Lucilla

Paronetto, Maria Paola

Parrales, Alejandro

Parri, Rhein

Parrington, John

Parrish II, Richard H.

Parrizas, Marcelina

Parry, Geraint

Parsons, Ryan G.

Partyka, Agnieszka

Parus, Anna

Pârvu, Marcel

Parzonko, Andrzej

Pasantes, Juan

Pasca, Sergiu

Pascal, Laura

Pascal, Steven M.

Paschalis, Eleftherios I.

Pasciu, Valeria

Pascual, Carmen María García

Pascual, María Belén
Pascut, Devis

Pasetto, Anna

Pashov, Anastas

Pasin, Fabio

Pasini, Luigi

Pasquariello, Rolando

Pasquet, Jean-Max

Pasquinelli, Gianandrea

Passali, Giulio Cesare

Passos, Marisa

Pasternak, Alexander

Pasternak, Taras

Pasternak, Taras P.

Pastey, Manoj

Pastor, William

Pastor-Anglada, Marçal

Pastore, Analisa

Pastores, Gregory M.

Pastorino, Paolo

Pastuch-Gawolek, Gabriela

Pasupuleti, Visweswara

Patarrão, Rita

Patching, Simon G

Pátek, Miroslav

Patel, Bhargav

Patel, Deven

Patel, Hardikkumar

Patel, Nibedita

Patel, Nilkanth

Patel, Nrupali

Patel, Rakesh

Patenge, Nadja

Pateras, Ioannis S.

Paternoster, Silvano

Pathak, Bhuvan

Pathak, Dhruba

Pathipati, Praneeti

Patik, Joradan

Patil, Deepak

Patil, Mahesh D.

Patil, Swati J.

Patila, Michaela

Patini, Romeo

Paton, Chad

Paton, Madison C. B.

Patra, Amiya

Patrauchan, Marianna A.

Patrick Lajoie, Patrick Lajoie

Patrignani, Paola

Patrinos, George

Patrizi, Annalisa 
Patrizi, Barbara

Patro-Małysza, Jolanta

Patrone, Cesare

Patruno, Cataldo

Patrussi, Laura

Patsali, Petros

Pattammattel, Ajith

Patten, Shunmoogum A.

Patterson, Jennifer

Patterson, Steven

Patti, Alessandro

Paudel, Keshav Raj

Paul, Ashim

Paul, Matthew

Paul, Parameswari

Paul, Sudip

Paul, Yaswen

Paulo, António

Paulusma, Coen C.

Pavanello, Chiara

Pavani, Krishna Chaitanya

Pavankumar, Theetha

Pavlidis, Efstathios

Pavlik, Edward

Pavlik, Edward J.

Pavlin, Matic

Pavlínková, Gabriela

Pavlos, Nathan John

Pavlov, Tengis

Pavlov, Youri I

Pavlovic, Nebojsa

Pavo, Noemi

Pavšič, Miha

Pawar, Shrikant D.

Pawar, Snehalata

Paweł'kowicz, Magdalena E

Pawelec, Graham

Pawlak, Aleksandra

Pawlak, Dariusz

Pawlak, Krystyna

Pawlak, Piotr

Pawlik, Andrzej

Pawlik, Anna

Pawlikowska-Pawlęga, Bożena

Pawlikowski, Marek

Pawlowski, Katharina

Pawłowski, Tomasz

Payne, Annette

Pazdro, Robert

Peacock, Christopher

Pearce, Neil
Pearce, Stephen

Pearl, Christopher A.

Pearring, Jillian

Pec, Martin

Pečenková, Tamara

Peche, Vivek

PECHEUR, Eve

Pecho-Vrieseling, Eline

Pecina-Slaus, Nives

Peck, Ammon

Pecori, Riccardo

Pecqueur, Claire

Pecyna, Paulina

Pedemonte, Nicoletta

Pedersen, Henrik

Pedeux, Rémy

Peditto, Matteo

Pedone, E. M.

Pedrazzini, Emanuela

Pedron, Sara

Pedrosa, Pedro

Pedroza-Garcia, Jose-Antonio

Peelman, Frank

Peeples, Mark

Peery, Rhiannon

Peeters-Scholte, Cacha

Peeva, Violeta

Pegolo, Enrico

Peifer, Christian

Peigneur, Steve

Peinado, María Ángeles

Peinelt, Christine

Peirats-Llobet, Marta

Peiró, Sandra

Peitsidis, Panagiotis

Peitzsch, Claudia

Peixoto, Cristina

Pejchal, Jaroslav

Pejin, Boris

Pekkanen-Mattila, Mari

Pěknicová, Jana

Pekrun, Arnulf

Peláez-García, Alberto

Pelagalli, Alessandra

Pellegrini, Marika

Pellerino, Alessia

Pelletier, Emilien

Pelliccioni, Paolo

Peluffo, Hugo

Peluso, Marco E. M.

Peña Quintana, Luis 
Peña, Cristina

Pena, Maria Marjorette

Peñafiel, Rafael

Peña-Llopis, Samuel

Penchev, Hristo

Pendin, Diana

Pendyala, Gurudutt N

Penezic, Ana

Peng, Chung-Kan

Peng, Congyue

Peng, Han

Peng, Hui

Peng, I-Chen

Peng, Xianjun

Peng, Xianlu Laura

Peng, $\mathrm{Xu}$

Peng, Yi-Jen

Peng, Ying-Jie

Peng, Ze

Peng, Zhao

Penke, Botond

Pennerman, Kayla

Pennicooke, Brenton

Penning, Louis

Pennisi, Elena Maria

Penno, Megan

Penson, Peter

Pentikäinen, Ulla

Pentzold, Stefan

Penzo, Marianna

Pepe, Jessica

Pepeu, Giancarlo

Perálvarez-Marín, Alex

Peran, Ivana

Perdas, Ewelina

Perdigão, Nelson

Perdomo, German

Perdomo, Jose

Perego, Carla

PEREIRA LEMOS, Julia

Pereira, Cidália Almeida

Pereira, Claudia

Pereira, Cláudia Maria F.

Pereira, David

Pereira, Filipe

Pereira, Florbela

Pereira, Leonel

Pereira, Leonor

Pereira, Maria De Lourdes

Pereira, Nelson A. M.

Pereira, Patricia
Pereira, Sofia

Pereira, Sónia

Perepechaeva, Maria L.

Perera, Chamini J.

Perera, Lalith

Perera, Omaththage

Pérez De La Vega, Marcelino

Pérez Puyana, Víctor Manuel

Pérez Tur, Jordi

Perez, Guillermo

Perez, Juan J.

Pérez, Ruth G.

Pérez, Salvador

Perez, Serge

Pérez-Badia, Rosa

Pérez-Caballero, Laura

Pérez-García, Selene

Perez-Hernandez, Concepción

Perez-Mendez, Oscar

Pérez-Pascual, David

Perez-Pe, Rosaura

Pérez-Ros, Pilar

Pérez-Sala, Dolores

Pérez-Sánchez, Carlos

Perez-Siles, Gonzalo

Perez-Stable, Carlos

Pérez-Torras, Sandra

Pérez-Vázquez, Victoriano

Pergament, Eugene

Periasamy, Ramesh

Perin, Cecilia

Periyasamy, Arun Prakash

Periyasamy, Palsamy

Perkeybile, Allison M.

Perkin, Lindsey C.

Perkins, Andrew

Perkovic, Ivana

Perkovic, Matea Nikolac

Perl, Andras

Perlikowski, Dawid

Perlin, Michael H.

Perluigi, Marzia

Permyakov, Eugene

Pernisová, Markéta

Pero, Raffaela

Peronnet, Frederique

Perouansky, Misha

Perreault, Jonathan

Perreault, Marie-Claude

Perrella, Giorgio

Perricone, Ugo 
Perrier, Jean-Francois

Perrino, Enrico

Perron, Hervé

Perrone, Anna Myriam

Perrone, Giancarlo

Perrone, Marco Alfonso

Perrone, Serafina

Perrucci, Gianluca Lorenzo

Perry, Rachel

Perše, Martina

Pershina, O. V.

Persico, Marco

Persson, Jenny

Perucca Orfei, Carlotta

Perucka, Irena

Perut, Francesca

Peruzzi, Francesca

Peruzzo, Roberta

Pes, Giovanni Mario

Peschke, Katharina

Pešić, Milica

Peška, Vratislav

Pest, Michael Andrew

Pestell, Richard

Pestov, Alexander

Pestov, Dimitri

Peták, István

Petejová, Nadežda

Péter, Beatrix

Peter, Francisc

Peter, Karlheinz

Peters, Donna

Peters, Judith

Peters, Maire

Petersen, Ole

Peterson, Larryn

Petersson, Kristoffer

Pethő, Zoltán

Petit, Patrice X.

Petitjean, Anne

Petra I., Lorenzo

Petrache, Irina

Petralia, Maria Cristina

Petreaca, Ruben C

Petrie-Hanson, Lora

Petrikaite, Vilma

Petrillo, Angelica

Petrillo, Sara

Petrinovic, Marija-Magdalena

Petrof, Basil J.

Petropoulos, Spyridon A
Petrosillo, Giuseppe

Petrosyan, Armen

Petrov, Alexey M.

Petrov, Anton

Petrova, Marina

Petrovic, Zeljka

Petrut, Bogdan

Petrzik, Karel

Petta, Ioanna

Petti, Carloalberto

Pettignano, Alberto

Petukhov, Dmitriy I.

Peulen, Olivier

Peverali, Fiorenzo

Peviani, Marco

Pey, Angel L.

Peyton, David H.

Pezzella, Alessandro

Pezzilli, Raffaele

Pezzuto, Aldo

Pfannschmidt, Thomas

Pfeffer, Lawrence M.

Pfeffer, Peter

Pfeffer, Ulrich

Pfeil, Alexander

Pfister, Barbara

Pham, Anh Tung

Pham, Thao

Phan, Thanh Kha

Phan, Thi Tuong Vy

Phang, James

Philipp, Manfred

Philips, Thomas

Phillips, Kevin J.

Phillips, Tycel

Philpott, Michael

Pi, Jiang

Piacenza, Elena

Piaggio, Giulia

Piane, Maria

Piasecka, Agnieszka

Piasecka, Małgorzata

Piątczak, Ewelina

Piatek, Jacek

Piatkevich, Kiryl D.

Piattelli, Adriano

Piazza, Francesco

Piazza, Gary A.

Piazzon, M. Carla

Picado, César

Picard, Didier 
Picca, Rosaria Anna

Piccinin, Elena

Piccionello, Antonio Palumbo

Piccirillo, Rosanna

Piccoli, Marco

Piccolo, Marialuisa

Pichler, Verena

Picimbon, Jean-François

Pickering, Andrew M.

Pickering, Raelene

Pico Alfonso, Antonio M.

Picollet-D'hahan, Nathalie

Picozza, Mario

Pidot, Sacha J.

Pidoux, Guillaume

Piechota, Aleksandra

Piechowicz, Lidia

Pieczonka, Adam M.

Piedras, Pedro

Piegat, Agnieszka

Piekiełko-Witkowska, Agnieszka

Piemonte, Fiorella

Pierce, David M.

Pierce, Dwight

Pierdomenico, Johnny Di

Piergentili, Roberto

Pieroni, Marco

Pierozan, Paula

Pierpaoli, Mattia

Pierreeiche, Olivier

Pierri, Ciro Leonardo

Pieters, Alejandro J.

Pieters, Roland

Pietras, Alexander

Pietropaolo, Adriana

Pietruszewska, Wioletta

Pietrzak, Bogusława

Pievani, Alice

Pignataro, Giuseppe

Pignatti, Patrizia

Pignone, Domenico

Pigossi, Suzane Cristina

Piiper, Albrecht

Pijnappel, W.W.M. Pim

Pikija, Slaven

Piknova, Barbora

Pikula, Konstantin

Pikuła, Michał

Pilarsky, Christian

Pilato, Fabio

Pileczki, Valentina
Piliponsky, Adrian M.

Pillai Raju, Raghavan

Pillozzi, Serena

Pilmane, Māra

Pilon, Marinus

Pilones, Karsten Anderson

Pilot, Guillaume

Pilot, Malgorzata

Pilu, Roberto

Piluso, Giulio

Piluzza, Giannella

Pilvankar, Minu

Pimentel, Madalena

Pimerzin, Aleksey

Pin, Fabrizio

Piña Capó, Maㅡ Nieves

Pina, Ana

Pina, João

Pinalli, Roberta

Piña-Oviedo, Sergio

Pinar, Anita

Pinart, Elisabeth

Pina-Vaz, Cidalia

Pindyurin, Alexey V.

Pineau, Pascal

Pineault, Nicolas

Pineda, Jose Ramon

Pinelli, Michele

Piñero, Manuel

Pingault, Lise

Pingtao, Ding

Pinho, Diana

Pini, Taylor

Pinna, Graziano

Pinnapireddy, Shashank Reddy

Pinniger, Gavin

Pino-Yanes, Maria

Pinter, Matthias

Pintilie, Grigore D.

Pinto Da Silva, Luís

Pinto, Deesy

Pinto, Gloria

Pinto, Mafalda

Pinto, Sara

Pinto-Medel, María

Pinzaru, Iulia

Piorecky, Marek

Piotrowska, Ewa

Piotrowska, Katarzyna

Piozzi, Antonella

Pippenger, Benjamin 
Piqueras, Laura

Pires, Bruno R. B.

Pirkmajer, Sergej

Piro, Rosario M.

Pirola, Luciano

Pirola, Yuri

Pirooznia, Mehdi

Pirozzi, Christopher J.

Pirtoli, Luigi

Pisani, Antonio

Pisani, Didier F.

Pisanu, Claudia

Pisanu, Maria Elena

Piscopo, Marina

Piscopo, Paola

Pisetsky, David S.

Pissas, Georgios

Pistis, Marco

Pistocchi, Anna Silvia

Pistone, Alessandro

Pita, Sebastian

Pitsillides, Andrew

Pitt, James

Pittalà, Valeria

Pittaluga, Anna

Pittman, Karin

Pitto, Letizia

Pitucha, Monika

Pitule, Pavel

Piu, Saha

Piunova, Victoria

Piva, Roberta

Piva, Terrence

Pivac, Nela

Pivarcsi, Andor

Pivoriunas, Augustas

Piwowarczyk, Katarzyna

Pizzanelli, Silvia

Pizzolanti, Giuseppe

Płachno, Bartosz

Placido, Antonio

Placido, Jersson

Placzek, William J.

Plakht, Ygal

Planas, Marta

Planeta, Diego

Plano, Laura De

Plant, Leigh

Plant, Timothy D.

Plante, Isabelle

Plastina, Pierluigi
Platania, Chiara

Plate, Lars

Platella, Chiara

Platt, Donna M.

Platta, Harald

Pławski, Andrzej

Playford, Martin P.

Płaza, Grażyna

Plaza-Zabala, Ainhoa

PŁażek, Agnieszka

Plazinski, Wojciech

Plazzer, John-Paul

Plech, Tomasz

Plecita, Lydie

Plenis, Alina

Pletser, Vladimir

Pletzer, Daniel

Plíhal, Ondřej

Plíhalová, Lucie

Ploetz, Evelyn

Ploner, Christian

Plonka, Przemyslaw

Plotka, Magdalena

Plotkin, Lilian I.

Plotnikov, Egor Y.

Płuciennik, Elżbieta

Pluta, Ryszard

Pluth, Janice M.

Pniewski, Tomasz

Pobbati, Ajaybabu

Pobiega, Katarzyna

Pocha, Christine

Pocheć, Ewa

Pochet, Roland

Pochwat, Bartłomiej

Pochynyuk, Oleh

Podar, Klaus

Poddelsky, Andrey

Podnar, Simon

Podobnik, Marjetka

Podust, Larissa M.

Poeggeler, Burkhard H.

Pogacnik, Lea

Poggi, Alessandro

Poggio, Paolo

Poglitsch, Marko

Pogocki, Dariusz

Pogorielov, Maksym

Pohjanvirta, Raimo

Pohjoismäki, Jaakko L. O.

Pohl, Carolina H. 
Pohl, Ehmke

Pohl, Sebastian Öther-Gee

Pohlkamp, Theresa

Poinsot, Verena

Poirot, Marc

Pojo, Marta

Póka, Róbert

Poku, Rosemary A.

Polacek, Norbert

Polak, Anna

Polak, Marta Ewa

Polakowski, Nicholas J.

Polańczyk, Andrzej

Polano, Maurizio

Polanski, Witold

Polari, Lauri

Poldermans, Don

Polge, Cécile

Poli, Alessandro

Poli, Giulia

Policar, Clotilde

Polidoros, Alexios

Polimeno, Lorenzo

Polina, Iuliia

Polini, Beatrice

Polishchuk, Pavel

Polishchuk, Roman

Polissidis, Alexia

Polit, Justyna

Politano, Luisa

Polizzi, Alessandro

Polkowska-Kowalczyk, Lidia

Pollastro, Stefania

Pollok, Karen E.

Polly, Patsie

Polticelli, Fabio

Poltronieri, Palmiro

Poluektova, Larisa

Polverino De Laureto, Patrizia

Polyakov, Nikolay

Pomastowski, Paweł

Pombo, Ana

Pomerantz, Ori

Pommerrenig, Benjamin

Pompella, Alfonso

Pompili, Elena

Poncet, Delphine

Ponnu, Jathish

Ponraj, Prabakaran

Pons, Miquel

Pontarotti, Pierre
Ponte, Nuno

Pontejo, Sergio M

Pontes, Bruno

Pontremoli, Carlotta

Poole, Angela Z.

Poole, Jill A.

Poole, Leslie

Poór, Péter

Pop, Laura Ancuța

Pop, Laurentiu

Pop, Oana Lelia

Popa, Lacramioara

Popa, Marcel

Popa, Mircea Ioan

Pope, Chad

Popeijus, Herman E.

Popelka, Hana

Popescu, Mihaela R.

Popgeorgiev, Nikolay

Popolo, Ada

Popova, Alexandra A.

Popova, Antoaneta

Popović Đorđević, Jelena

Popp, Henning

Poppinga, Simon

Poradowski, Dominik

Porcheri, Cristina

Porchia, Marina

Pordea, Anca

Porokhovnik, Lev

Porollo, Aleksey

Porras, Ignacio

Porrelli, Davide

Porres, Jesus

Porrini, Esteban L.

Porro, Chiara

Porta, Chiara

Porta, Helena

Portelli, Marco

Porteu, Françoise

Portillo-Lara, Roberto

Poschenrieder, Charlotte

Posey, Avery D.

Pospíšilová, Šárka

Possidente, Bernard

Possik, Elite

Postler, Thomas S.

Potaczek, Daniel P

Potaniec, Bartłomiej

Potekhin, Alexey

Potenza, Nicoletta 
Potes, Yaiza

Potiron, Vincent

Potokar, Maja

Potrykus, Katarzyna

Potter, Huntington

Potter, Julia

Potter, Pamela E

Poudel, Barun

Poudel, Sher Bahadur

Pouliot, Roxane

Pourbaghi Masouleh, Milad

Pourcet, Benoît

Pourié, Grégory

Pourquier, Philippe

Pourrezaei, Kambiz

Povelones, Megan

Powers, Evan

Powrózek, Tomasz

Poyet, Jean-Luc

Poynter, Sarah J.

Pozharskiy, Edvin

Poznański, Jarosław

Pozzi, Cecilia

Pozzi, Nicola

Pozzo, Eleonora Da

Pozzobon, Michela

Prabhu, Antony Herold

Prabhu, Lakshmi

Prada, Ilaria

Pradhan, Dinesh

Pradhan, Sriharsa

Prado-Gotor, Rafael

Pradotto, Luca Guglielmo

Prakapenka, Alesia

Prakash, Nilima

Prakash, Satya

Pramanik, Arindam

Pramanik, Avijit

Pramod, Sreepriya

Pranckeviciene, Erinija

Pranno, Nicola

Prasad, Ankush

Prasad, Asuri N.

Prasad, Chitra

Prasad, Kasavajhala

Prasai, Anesh

Prassas, Ionassis

Prassl, Ruth

Prat, Maria

Pratesi, Alessandro

Pratsinis, Harris
Prattichizzo, Francesco

Prchal, Josef T.

Preca, Bogdan Tiberius

Predescu, Dan

Prehn, Jochen H. M.

Prehna, Gerd

Prêle, Cecilia M.

Preller, Matthias

Premilovac, Dino

Prerostova, Mgr. Sylva

Pressyanov, Dobromir

Prestel, Matthias

Prestori, Francesca

Prestwich, Erin

Prevette, Lisa E.

Prévost, Michel

Preziosa, Paolo

Preziosi, Daniele

Priault, Muriel

Pribyl, Jan

Price, Jeff

Price, Patricia

Priel, Avi

Priestman, David A.

Prieto, Elena

Prieto, Rafael Maria

Prigge, Matthias

Primack, William A.

Prinsi, Bhakti

Printza, Nikoleta

Priola, Emanuele

Prior, Timothy J.

Pritchett, James

Pritzker, Kenneth

Privette Vinnedge, Lisa

Procházková, Jiřina

Prochownik, Edward V.

Prodromos, Chadwick C.

Proescholdt, Martin

Proestos, Charalampos

Proestou, Dina A.

Proia, Richard

Proietti, Silvia

Prokop, Jeremy W

Prömel, Simone

Pronin, Alexander V.

Prosperi, Ennio

Prosperi, Jenifer

Proszynski, Tomek

Protasi, Feliciano

Protasoni, Marina 
Protogerou, Athanase D.

Protonotarios, Alexandros

Proukakis, Christos

Proulx, Steven

Provot, Sylvain

Prudovsky, Igor

Pruess, Birgit M.

Prugovečki, Biserka

Pruneda, Jonathan N.

Prystupa, Andrzej

Przekora, Agata

Przepiera-Będzak, Hanna

Przybył, Jarosław

Przybył, Jarosław L.

Przybyło, Małgorzata

Przygodzki, Tomasz

Pshezhetsky, Alexey

Psilodimitrakopoulos, Sotiris

Pszczółkowska, Agnieszka

Puca, Annibale

Pucci, Marzia

Pucciarelli, Sandra

Puchades, María Jesús

Puchalka, Radoslaw

Puchkova, Ludmila V.

Puente-Massaguer, Eduard

Puerta, Elena

Puertas, Maria Carmen

Puglia, Giuseppe

Puglisi, Fabio

Puigdomènech, Pere

Puiggalí, Jordi

PUILLANDRE, Nicolas

Puła, Bartosz

Pula, Giordano

Pulaski, Lukasz

Pulavarti, Surya VSRK

Pulford, Karen

Pulgar, Victor

Pulicharla, Rama

Pulido, Olga M

Pulido, Rafael

Pulit-Prociak, Jolanta

Pullen, Nicholas

Pulliero, Alessandra

Punetha, Ankita

Pungerčar, Jože

Pupilli, Fulvio

Puppala, Naveen

Puri, Sonam

Purpura, Valeria
Purrucker, Jan Christoph

Pusch, Stefan

Püschel, Gerhard

Pushchina, Evgeniya

Pussinen, Pirkko

Pusztahelyi, Tünde

Putnik, Predrag

Puvvula, Pavan Kumar

Puzer, Luciano

Pycia, Karolina

Pyo, Jung-Soo

Pytel, Dariusz

Pyun, Jae-Cheol

Pyzik, Michal

Qadri, Syed

Qasim, Muhammad

Qasim, Syed Saad B

Qi, Junpeng

Qi, Yanfei (Jacob)

Qian, Jin

Qiao, Pengfei

Qimron, Udi

Qin, Qian

Qin, Tingting

Qin, Xian-Yang

Qin, Zhaohai

Qin, Zhenpeng

Qin, Zhihui

Qiu, Bin

Qiu, Yongjian

Qiu, Zhen

Qu, Ning

Qu, Peng

Quadri, Marika

Quadri, Syed A.

Quadros, Edward

Quagliariello, Vincenzo

Quaglino, Daniela

Quaglio, Deborah

Quaini, Federico

Quang Le, Bach

Quarck, Rozenn

Quartu, Marina

Quattrocelli, Mattia

Quax, Paul

Quek, Camelia

Queralt, Ethel

Querleu, Denis

Quéro, Anthony

Quesada Pérez, Víctor

Quesnel-Vallières, Mathieu 
Quigley, Raymond

Quinn, John P.

Quinn, Peter M.J.

Quintana, José

Quintana, Mercedes

Quintanilla, Luis G

Quintas, Alexandre

Quintas, Luis Eduardo M.

Quinzii, Catarina Maria

Quiroga, Cecilia

Rabanal Ruiz, Yoana

Rabiee, Hesamoddin

Rabuazzo, Agata Maria

Raccuia, Salvatore Antonino

Rachmawati, Dessy

Rachon, Dominik

Racioppi, Marco

Racz, Attila

Racz, Ildiko

Radaram, Bhasker

Radchenko, Eugene V.

Radchuk, Volodymyr

Radcliff, Fiona Jane

Rademaker, Gilles

Radic, Marco

Radić, Tomislav

Radichev, Ilian

Radisavljevic, Ziv

Rádis-Baptista, Gandhi

Radko, Lidia

Radosavljević, Tatjana

Radošević-Stašić, Biserka

Radosinska, Jana

Radu, Beatrice Mihaela

Radu, Fierascu

Radzikowska, Urszula

Rae, Colin

Rafael, Diana

Rafi, Junaid

Rafiad, Islam

Rafiq, Kazi

Ragavan, Mukundan

Ragazzi, Eugenio

Rageul, Julie

Raggi, Carla

Raghunathan, Vijay

Ragino, Yuliya I.

Ragnini-Wilson, Antonella

Ragno, Rino

Ragusa, Andrea

Ragusa, Maria Antonietta
Ragusa, Michael J.

Rahat, Milki

Rahbar, Afsar

Rahbari, Nuh N.

Rahikainen, Moona

Rahimi, Farid

Rahman, Ashiqur X

Rahman, M. Azizur

Rahman, Md. Atikur

Rahman, Milladur

Rahman, Mohammad Mijanur

Rahman, Safikur

Rahmani, Farzin

Rahnavard, Gholamali

Rai, Krishan

Rai, Navneet

Rai, Sumit

Rai, Tatemitsu

Rai, Vikrant

Rai, Vineeta

Raikar, Sunil S.

Raikes, Adam C.

Raimondo, Annunziata

Raimondo, Stefania

Raina, Deepak

Raina, Komal

Rainger, George Edward

Rajagopalan, Anugraha

Rajakumar, Nagalingam

Rajalingam, Krishnaraj

Rajamohan, Arun

Rajas, Fabienne

Rajasekharan, Satish Kumar

Rajendran, Jagathesh Chandra

Rajendran, Vazhaikkurichi

Rajesh, Ramachandran

Rajkowska, Katarzyna

Rajnarayanan, Rajendram

Rajpurohit, Surendra

Rajput, Sandeep

Raju, Hariharan

Rak, Janusz

Rakic, Jelena

Rakonczay, Zoltán

Rakotondrafara, Aurelie

Rakus, Dariusz

Ralle, Martina

Ralli, Massimo

Ramachandran, Srinivasan

Ramadas, Amutha

Ramadori, Giuliano 
Ramalho Venâncio, Anabela Santo

Ramalho, Maria João

Ramalho-Santos, João

Ramalingam, Nagendran

Ramalingam, Naveen

Ramamurthy, Narayan

Raman, Lennart

Ramani, Komal

Ramani, Kritika

Ramani, Vijay

Ramaraj, Pandurangan

Ramasamy, Ranjan

Ramasubramanian, Anand Anand

Ramaswami, Mani

Ramazzotti, Matteo

Rambow, Florian

Ramchandani, Divya

Ramegowda, Venkategowda

Ramesh, Aramandla

Ramesh, Sunita A.

Ramezanpour, Mahnaz

Ramić, Snježana

Ramil, Carlo

Ramírez Sánchez, Manuel

Ramírez, Manuel

Ramírez, Martín

Ramírez, Sara

Ramírez-Gonzalez, Ricardo H.

Ramírez-López, María Teresa

Ramírez-Parra, Elena

Ramirez-Peña, Esmeralda

Ramos Cabrer, Pedro

Ramos Morales, Francisco

Ramos, Carmen

Ramos, Catarina

Ramos, Sonia

Ramos-Garcia, Pablo

Rampazzo, Elena

Rampias, Theodoros

Rampon, Christine

Ramsköld, Daniel

Ran, Sophia

Ranawade, Ayush

Ranc, Václav

Rancan, Fiorenza

Rand, Matthew

Randall, Stephen K.

Randall-Demllo, Sarron

Randazzo, Basilio

Randow, Felix

Ranganathan, Natarajan
Ranganathan, Parvathi

Ranganathan, Srivathsan

Rangappa, Raju Bheemanahalli

Rangasamy, Sampath

Rangel-Barajas, Claudia

Rangnekar, Abhijit

Ranieri, Danilo

Ranieri, Federico

Ranieri, Marianna

Ranjan, Kishu

Ranjit, Suman

Rao, C.V.

Rao, Feng

Rao, Ponugoti Vasantha

Rao, Vidhya

Rapado-González, Óscar

Rapak, Andrzej

Rapala-Kozik, Maria

Raphael, Itay

Rapino, Cinzia

Rapp, Alexander

Rapposelli, Simona

Rapson, Trevor D.

Rascio, Agata

Rashad, Adel Ahmed

Rashdan, Nabil

Rashid, Mohammad Harun

Rašin, Mladen Roko

Raskina, Olga

Rasmussen, Soren

Rasoulianboroujeni, Morteza

Rastelli, Giulio

Rastenytė, Daiva

Rastrelli, Luca

Rat, Patrice

Ratajewski, Marcin

Rateb, Mostafa

Ratera, Imma

Rathbone, Alex J.

Rather, Gulam

Rathinavelu, Appu

Rathmacher, John A.

Ratiu, Andreea

Rato, Luís Pedro Ferreira

Rätsep, Matthew

Rattan, Ramandeep

Rattray, Zahra

Rau, Ileana

Raucci, Maria Grazia

Raucci, Umberto

Rauceo, Jason M. 
Rauch, Bernhard H.

Rauch, Frank

Rauch, Jens

Raudino, Antonio

Raudsepp, Terje

Rauova, Lubica

Rauvala, Heikki

Raval, Manmeet

Ravanel, Stephane

Ravanidis, Stylianos

Ravegnini, Gloria

Ravera, Enrico

Ravera, Mauro

Ravi, Anuradha

Ravi, Rama S.

Ravin, Nikolai V.

Ravingerová, Táňa

Ravnik-Glavač, Metka

Ravuri, Sudheer

Rawat, Manmeet

Rawla, Prashanth

Ray, Samriddha

Ray, Sutapa

Raya, Angel

Rayalam, Srujana

Raychaudhuri, Pradip

Rayes, Julie

Raynaud, Cécile

Rayport, Stephen

Ražná, Katarína

Razvan-Cosmin, Petca

Razzaque, Samsad

Razzuoli, Elisabetta

Read, David Alan

Reader, Jocelyn C.

Reader, Karen L.

Reagan, John L

Reale, Marcella

Reali, Eva

Ream, Walt

Rebbeck, Robyn

Rébé, Cédric

Rebelo, Sandra

Rebmann, Vera

Rebuli, Meghan

Rebuzzini, Paola

Recalde, Sergio

Recasens, Ariadna

Rechkunova, N. I.

Reddi, Amit R.

Reddy, Pullagurala Venkata Laxma
Reddy, Sakamuri

Reddy, Sudhir Putty

Redell, John

Redina, Olga

Redmer, Torben

Redolfi, Michaël

Redondo, Maximino

Redowicz, Maria Jolanta

Reed, May J.

Reed, William R.

Reers, Stefan

Reeve, Vivienne

Reeves, Stephen R.

Regal, Jean

Regazzo, Daniela

Regiec, Andrzej

Reglödi, Dóra

Régnier-Vigouroux, Anne

Reguła, Julita

Regunath, Hariharan

Rehfeld, Jens F.

Rehman, Atteeq Ur

Rehman, Michael

Rehrig, Erin

Reichenbach, Alex

Reichert, David E.

Reid, Brian

Reid, Christopher A.

Reif, Shimon

Rein, Theo

Reinach, Peter S.

Reinartz, Silke

Reiner, Agnes

Reinhard, Jacqueline

Reinhardt, Christoph

Reinke, Hans

Reis, Henning

Reis, Sara

Reiser, Jakob

Reiser, Peter J.

Reiss, Allison B

Reiter, Ofer

Relat, Joana

Relic, Biserka

Religa, Piotr

Rembiałkowska, Ewa

Rembold, Christopher

Remelli, Maurizio

Remigante, Alessia

Remuzzi, Andrea

Ren, Jun 
Ren, Ping

Ren, Yuan

Ren, Zhenhua

Renaud, Stephen

Renault, Nicolas

Renčiuk, Daniel

Rengaraj, Deivendran

Rengasamy, Kannan

Rengo, Sandro

Renshaw, Derek

Renu, Sankar

Renukuntla, Jwala

Renzi, Samuele

Renzini, Alessandra

Renzoni, Adriana

Requena, Jesus

Requicha, Joao

Resch, Ulrike

Rescifina, Antonio

Resentini, Francesca

Reshkin, Stephan

Reshkin, Stephan Joel

Restivo, Francesco Maria

Retière, Christelle

Rettinger, Eva

Rety, Stephane

Retzer, Katarzyna

Reuter, Klaus

Reuter, Sebastian

Revel-Vilk, Shoshana

Reverter, David

Revuelta, Julia

Révy, Patrick

Rewitz, Kim F.

Reychler, Hervé

Reyes Parada, Miguel

Reyes-del Valle, Jorge

Reyes-Garcés, Nathaly

Reyes-López, Felipe E.

Reyes-Ortega, Felisa

Reynisson, Jóhannes

Reynoird, Nicolas

Reynolds, C. Patrick

Reynolds, Clare M.

Reynolds, Paul

Řezáčová, Martina

Rezania, Shahabaldin

Režen, Tadeja

Rezk, Bashir M.

Reznikov, Leah R.

Rhaleb, Nour-Eddine
Rhein, Mathias

Ribaldone, Davide

Ribaldone, Davide Giuseppe

Ribaudo, Giovanni

Ribeiro, Artur

Ribeiro, Artur Jorge Araújo Magalhães

Ribeiro, César Augusto João

Ribeiro, Isabel

Ribeiro, Viviana P.

Ribeiro-Barros, Ana I.

Ribera, Jordi

Ribero, Simone

Ricagno, Stefano

Ricardo, Sara

Riccardi, Claudia

Riccardo, Zenezini Chiozzi

Ricci, Claudia

Ricci, Federico

Ricci, Giulia

Ricciardelli, Carmela

Ricciardi, Loredana

Ricciardi, Maria Rosaria

Riccio, Antonella

Riccio, Gennaro

Riccio, Paolo

Ricci-Vitiani, Lucia

Richard, Bodnar

Richard, Melissa

Richard, Sabine

Richard, Steet

Richards, Dylan J.

Richards, Joanne S.

Richardson, Lauren

Richardson, Sandra R.

Richardson, Simon C. W.

Riches-Suman, Kirsten

Richter, Katharina

Richter, Katrin

Richter, Manuel Jonas

Richter, Peter

Richter, Shachar

Richter, Susan

Richter, Wito

Richtera, Lukas

Richter-Dennerlein, Ricarda

Riddiford, Lynn M.

Rideout, Elizabeth J.

Ridola, Lorenzo

Rieder, Leila E.

Riedl, Rainer

Riese, Matthew J. 
Rietdorf, Katja

Riffo-Vasquez, Yanira

Rigalli, Juan Pablo

Riganti, Chiara

Rigas, Stamatis

Righetti, Laura

Righi, Alberto

Righi, Marco

Rigo, Riccardo

Rigual, Victoria

Rihan, Hail

Rijavec, Matija

Rikkerink, Erik

Rilcheva, Violeta S.

Rim, John Hoon

Rim, Kyung Taek

Rimoldi, Isabella

Rimondi, Erika

Rimondini, Lia

Rimpelova, Silvie

Rimpelová, Silvie

Rinaldi, Fabio

Rincon, Melvin Y.

Ringli, Christoph

Rinschen, Markus M.

Riobo, Natalia

Rios, Francisco

Rios-Covian, David

Ríos-Santos, Jose Vicente

Ripperger, Jürgen A.

Ris, Laurence

Risk, Janet M.

Riska, Paul

Risner, Michael

Ritchie, Helena

Ritsma, Laila

Ritter, Andreas

Riuzzi, Francesca

Riva, Antonella

Riva, Federica

Rivera, Ernesto

Rivera, Hugo Vara

Rivera, Víctor M.

Rivera-Monroy, Zuly Jenny

Rivero, Francisco

Rivinius, Rasmus

Rižner, Tea Lanišnik

Rizos, Dimitrios

Rizvanov, Albert

Rizvi, Syed A. A.

Rizzi, Chiara
Rizzi, Laura

Rizzi, Manuela

Rizzi, Menico

Rizzo, Alessandro

Rizzo, Fabiana

Rizzo, Paola

Ro, Hyeon-Su

Ro, Hyunju

Ro, Seung-Hyun

Roana, Janira

Roato, Ilaria

Robakowski, Piotr

Robaszkiewicz, Agnieszka

Robben, Johan

Robert, Philippe

Roberto, Silvana

Roberto, Vânia

Roberts, David

Roberts, Tara

Robertson, Cayleih E.

Robin, Arif Hasan Khan

Robin, Jérôme D.

Robinson, Anne Skaja

Robinson, Douglas

Robinson, Karl

Robinson, Seri

Robles, Vanesa

Robles-Marhuenda, A.

Roby, Katherine

Roca, Joaquim

Rocca, Carlo Della

Rocca, Roberta

Roccaro, Aldo

Rocha, Javier

Rochais, Christophe

Rocha-Martin, Javier

Rocha-Rodrigues, Sílvia

Roche, Benjamin

Roche, Marc De La

Roche, Philippe

Rocher, Asuncion

Rochfort, Keith

Roda, Aldo

Rodamilans, Bernardo

Rodat-Despoix, Lise

Rodella, Luigi F.

Rodemer, William

Roderburg, Christoph

Rodet, Franck

Rodgers, Jean

Rodilla, Verónica 
Rodin, Andrei S.

Rodolfo, Monica

Rodrigo, Guillermo

Rodrigo, Ismael

Rodrigo-Muñoz, José M

Rodriguero, Marcela

Rodrigues, António S.

Rodrigues, Célia F.

Rodrigues, Francisca

Rodrigues, Joana

Rodrigues, Márcia T.

Rodrigues, Olivier

Rodrigues, Pedro Miguel

Rodrigues, Teresa

Rodríguez González, Álvaro

Rodriguez Lozano, Francisco Javier

Rodriguez Sosa, Jose $\mathrm{R}$

Rodriguez Valle, Manuel

Rodríguez Villanueva, Javier

Rodriguez, Analiz

Rodriguez, Cristian

Rodríguez, Isabel

Rodríguez, Matías Morín

Rodriguez, Patricia

Rodríguez, Ricardo Reyes

Rodríguez, Víctor Manuel

Rodriguez-Cueto, Carmen

Rodriguez-Gil, Jorge

Rodríguez-Jerez, José Juan

Rodríguez-López, Lorena

Rodriguez-Lorenzo, Laura

Rodríguez-Lozano, Francisco Javier

Rodríguez-Pallares, Jannette

Rodríguez-Perálvarez, Manuel

Rodríguez-Sanz, David

Rodriguez-Sinovas, Antonio

Rodriguez-Vargas, Jose M.

Rodriguez-Vita, Juan

Rodríguez-Yoldi, María Jesús

Rodriquez, Manuela

Rodziewicz-Motowidło, Sylwia

Roe, Richard Michael

Roedel, Franz

Roelen, Bernard

Roex, Gils

Rogalska, Justyna

Rogatzki, Matthew

Roger, Lauréline

Rogers, Hilary

Rogina, Anamarija

Rogobete, Alexandru Florin
Rogozea, Liliana

Rogozhin, Eugene

Roh, Sangho

Rohacs, Tibor

Rohan, Fernando M.

Rohde, John R.

Rohila, Jai

Rohila, Jai S.

Roizenblatt, Marina

Rojano-Delgado, Antonia Maria

Rojo Marcos, Francisco Javier

Rojo, Maria Angeles

Rokavec, Matjaz

Rokosh, Donald G.

Rokstad, Anne Mari

Rolbiecki, Leszek

Roldo, Marta

Rollenhagen, Christiane

Roller, Richard J.

Rom, Slava

Roman, Shaun

Romanelli, Alessandra

Romanello, Vanina

Romanenko, Svetlana A.

Romano, Alessandra

Romano, Giovanni Luca

Romano, Lorenzo

Romano, M Carmen

Romano, Marco

Romano, Nicla

Romanov, Georgy A.

Romanov, Roman A.

Romański, Jarosław

Romão, Luísa

Roma-Rodrigues, Catarina

Romero Morales, Carlos

Romero, Fernando M.

Romero, Irene

Romero, Julian

Romero, Marta R.

Romero-Lorca, Alicia

Romero-Reyes, Marcela

Ronca, Roberto

Roncada, Paola

Roncevic, Tomislav

Ronchetti, Simona

Roncucci, Luca

Rondanino, Christine

Ronellenfitsch, Michael W.

Rong, Chan Kuan

Rongvaux, Anthony 
Roni, Monzurul

Roninson, Igor

Ronnberg, Elin

Ronquist, Gunnar

Ronzan, Marilena

Ronzitti, Giuseppe

Roquis, David

Rosa, Arianna Carolina

Rosa, Bruce A.

Rosa, Irene

Rosado, Carlos

Rosado, Juan

Rosa-Garrido, Manuel

Rosales, Ruben

Rosas-Hernandez, Hector

Rosati, Jessica

Rosato, Antonio

Roscoe, Thomas

Rose, Christopher F.

Roseanu Constantinescu, Anca

Roseanu, Anca

Rose-John, Stefan

Roselli, Marianna

Rosen, Michael J.

Rosendale, Andrew J.

Rosenfeld, Mark

Rosengren, Johan

Rosenkranz, Jeremy Amiel

Rosenthal, Cynthia

Rosenthal, Rita

Rosenzweig, Steven A.

Roshan, Amit

Roshanghias, Ali

Rosic, Gvozden

Rosicka-Kaczmarek, Justyna

Rösl, Frank

Ross, Ian

Ross, Matthew

Ros-Santaella, José Luis

Rossbach, Oliver

Rossetti, Carlo

Rossi, Andrea

Rossi, Barbara

Rossi, Filomena

Rossi, Francesca

Rossi, Giulio

Rossi, Michela

Rossi, Miriam

Rossi, Noreen F.

Rossi, Paolo

Rossi, Pellegrino
Rossignol, Julien

Rosso, Chiara

Rosspeintner, Arnulf

Rossy, Jérémie

Roszak, Pawel

Roszczenko-Jasinska, Paula

Roterman, Irena

Roth, Michael

Rothbauer, Mario

Rothenberg, Richard

Rothhammer, Veit

Rotili, Dante

Rotoli, Bianca Maria

Rotolo, Caterina

Rotondo, John Cahrles

Rouault-Pierre, Kevin

Roudier, Emilie

Roue, Gael

Rouge, Pierre

Rougeot, Julien

Rouhiainen, Ari

Rouillé, Yves

Rouleau, Etienne

Rouleau, Matthieu

Rousar, Tomas

Rousselle, Patricia

Rousset, Francis

Routhu, Nanda Kishore

Routray, Pratyush

Roux, Stanley J.

Rovatsos, Michail

Rovere, Mauro

Roversi, Pietro

Rovida, Elisabetta

Rovina, Davide

Rowe, Glenn C.

Rowley, Paul Andrew

Roy, Anuradha

Roy, Dipankar

Roy, Ipsita

Roy, Partha

Roy, Saktimayee Mitra

Roy, Soumen

Roy, Swapan Kumar

Roy, Urmi

Roychowdhury, Rajib

Royo, Felix

Różalska, Barbara

Różalska, Sylwia

Rozalski, Marcin

Rozhon, Wilfried 
Rozov, Serge M.

Różycki, Bartosz

Rozza, Ariane

Ruan, Ke

Ruaro, Barbara

Rubasinghege, Gayan

Rubattu, Speranza

Rubin, Robert L.

Rubina, Kseniya

Rubinowska, Katarzyna

Rubio, Myriam

Rubio-Aliaga, Isabel

Rubio-Cabetas, M.J.

Rubis, Blazej

Rubis, Pawel

Rubtsov, Nikolay

Rucci, Nadia

Ruch, Randall

Rucinski, Marcin

Rucker, Robert B.

Ruden, Douglas

Rudnicka, Wiesława

Rudnóy, Szabolcs

Rudolf, Emil

Rudolph, Uwe

Rudra, Arnab

Rudroff, Thorsten

Rudzińska, Magdalena

Rueda, Bo

Ruegg, Joelle

Ruegg, Urs

Ruest, L. Bruno

Ruffinatti, Federico A

Ruffinatti, Federico Alessandro

Ruffner, Melanie A.

Ruffolo, Gabriele

Rufini, Alessandro

Ruggieri, Vitalba

Rugolo, Michela

Ruhanya, Vurayai

Rühl, Martin

Ruivo, Raquel

Ruiz De Mier, M. Victoria Pendón

Ruiz Laza, Rocío

Ruiz Ojeda, Francisco Javier

Ruiz Téllez, Trinidad

Ruiz, Facundo

Ruiz, Mario

Ruiz, Matthieu

Ruiz-Rodríguez, Juan C.

Ruiz-Ruano, Francisco J.
Ruiz-Valdivieso, Victor

Ruiz-Velasco, Victor

Rummel, Andreas

Rumyantseva, Natalya

Rund, Deborah

Ruppert, Elisabeth

Rurek, Michal

Rusak, Malgorzata

Rusanova, Iryna

Rusciano, Dario

Rusciano, Maria Rosaria

Rusinek, Dagmara

Rusmini, Paola

Rusnati, Marco

Russ, Brendan

Russell, Rod

Russell, Scott

Russi, Sabino

Russo, Alessandro

Russo, Annapina

Russo, Chiara

Russo, Daniela

Russo, Domenico

Russo, Ethan

Russo, Francesco Paolo

Russo, Giulia

Russo, Isabella

Russo, Maria

Russo, Massimo

Russo, Matteo A.

Russo, Nino

Russu, Wade

Rusu, Laura Cristina

Rusu, Mugurel Constantin

Rusu, Teodor

Ruszkowska-Ciastek, Barbara

Ruthstein, Sharon

Rutigliano, Grazia

Rutkowski, Joseph M.

Rutters, Femke

Rutzler, Michael

Ruvo, Menotti

Ruysschaert, Jean-Marie

Růzǐčka, Kamil

Ruzza, Paolo

Ruzzene, Maria

Ryabchikova, Elena I.

Ryadnov, Max

Rybak, Leonard P.

Rybka, Jakub Dalibor

Rybka, Krystyna 
Rybtsov, Stanislav

Rychahou, Piotr

Rychkov, Denis

Rychkov, Grigori

Rychlik, Blazej

Rymowicz, Waldemar

Ryota, Tamura

Ryszawy, Damian

Ryszkowska, Joanna

Rytelewski, Mateusz

Ryu, Changseon

Ryu, Jiyoon

Ryu, Jung Su

Ryu, Kwon-Yul

Ryu, Sangjin

Rzemieniec, Joanna

Rzepecka-Stojko, Anna

Rzepkowska, Malgorzata

Rzewuska, Magdalena

Rzońca, Sylwia Olimpia

Rzymowska, Jolanta

Rzymski, Piotr

Sá, Rosália

Saad, Nizar Y.

Saakes, Michel

Saavedra, Juan M.

Saba, Nakhle S.

Sabater, Bartolome

Sabatino, David

Sabatino, Leo

Sabhachandani, Pooja

Sabina, Saverio

Sabnis, Himalee

Sabogal-Guáqueta, Angélica María

Sabol, Maja

Šabovič, Mišo

Saccà, Sergio Claudio

Saccenti, Edoardo

Sacco, Pasquale

Sacco, Rodolfo

Saccone, Salvatore

Saccone, Valentina

Sachar, Madhav

Sacher, Edward

Sachio, Tsuchida

Sachithanandham, Jaiprasath

Sachkova, Maria

Sacitharan, Pradeep Kumar

Sack, Ulrich

Saclier, Marielle

Saczewski, Jarosław
Saczonek, Agnieszka Owczarczyk

Sadaghianloo, Nirvana

Sadasivam, Mohanraj

Saddala, Madhu Sudhana

Sader, Robert

Sadhukhan, Ayan

Sadiku, Pranvera

Sadler, Anthony

Sadovsky, Yoel

Sadri-Ardekani, Hooman

Sadykov, Marat R.

Saeki, Kumiko

Sáez, Guillermo T.

Safa, Ahmad R.

Safo, Martin K.

Safronova, Tatiana

Sagheddu, Claudia

Sagner, Andreas

Sagnou, Marina

Sah, Nirnath

Saha, Dipongkor

Saha, Nirmalya

Saha, Ranajay

Sahab, Sareena

Saharan, Lalita

Saheki, Takeyori

Sahiner, Nurettin

Sahni, Sanjeev K.

Sahoo, Pabitra

Sahu, Debashish

Sahu, Ravi P

Saielli, Giacomo

Saif, Sadia

Saika-Voivod, Ivan

Saini, Ramesh Kumar

Saini, Shikha

Saint-Dizier, Marie

Saint-Pol, Julien

Sainz, Rosa M

Saito, Izumu

Saito, Kan

Saito, Mariko

Saito, Ryoko

Saito, Yoshiro

Saito, Yukihiro

Saitoh, Akiyoshi

Saitoh, Issei

Saitoh, Masao

Saiz-Fernández, Iñigo

Saiz-Sanchez, Daniel

Sajesh, Babu V. 
Sajeva, Maurizio

Sak, Jarosław J.

Sakabe, Masahide

Sakač, Nikola

Sakai, Atsushi

Sakai, Hideki

Sakai, Hiromi

Sakai, Hiromichi

Sakai, Hiroyasu

Sakakibara, Iori

Sakakibara, Keiko

Sakamoto, Akio

Sakamoto, Kenji

Sakamuri, Siva Sankara Vara Prasad

Sakamuro, Daitoku

Sakane, Fumio

Sakata, Junki

Sakata, Naoaki

Sakellari, Dimitra

Saki, Morteza

Saki, Najmaldin

Sakina, Mhaouty-Kodja

Sako, Kaori

Sakr, Soulaiman

Saksena, Nitin

Saksena, Seema

Sakthivel, Kogularasu

Sakurai, Hidetoshi

Sakurai, Kenichi

Sakurai, Yu

Sala, Gessica

Sala, Lucia La

Saladi, Srinivas Vinod

Salagierski, Maciej

Saláková, Martina

Salamanca, Eisner

Salamon, Sylwia

Salánki, Katalin

Salas, Joaquín J.

Salas-Huetos, Albert

Salazar, Ivan

Salazar, Juan J.

Saldarelli, Pasquale

Saleem, Ayesha

Saleem, Fozia

Saleh, Fatima A.

Saleh, Mona

Salehi, S Albert

Salehi, Sarah

Salehzadeh-Yazdi, Ali

Salem, Abdelhakim
Salem, Victoria

Salemi, Michele

Saler, Marco

Salerno, Alessandro Gonzalez

Sales, Ester

Sales-Gil, Raquel

Salgar, Shashikumar

Salifoglou, Athanasios

Salim, Vonny

Salimi, Zahra

Salinska, Elzbieta

Sallam, Ahmad

Salmeri, Mario

Salmhofer, Hermann

Salmina, Alla

Salmon, Morgan

Salomaa, Sisko

Salomon, Johanna

Salonurmi, Tuire

Salpini, Romina

Salsi, Valentina

Salta, Evgenia

Saltarella, Ilaria

Salton, Francesco

Salucci, Sara

Saluk-Bijak, Joanna

Salunke, Smita

Salvador F, Aliño

Salvadori, Nicola

Salvarani, Nicolo'

Salvati, Erica

Salvato, Fernanda

Salvatore, Francesco

Salvatore, Lisa

Salvatore, Maria Michela

Salvi, Mauro

Salvi, Silvia

Salzer, Isabella

Sam, Ramin

Samaja, Michele

Samal, Sangram

Samal, Sneha

Samanta, Pralok Kumar

Sambandam, Vaishnavi

Šamec, Dunja

Samhan-Arias, Alejandro

Samiec, Marcin

Samigullin, Tahir H.

Samir, Parimal

Sammar, Marei

Sammarco, Giuseppe 
Samochowiec, Jerzy

Sampaolo, Simone

Sampath, Chethan

Sampath, Karuna

Samson, Abraham O.

Samsonov, Sergey

Samsudin, Asep Muhamad

Samuel, Samson Mathews

Samuels, A. Lacey

Samuels, Jon Dean

Samulski, Richard Jude

San Bautista, Alberto

Sanabria, Hugo

Sanak, Marek

Sanche, Leon

Sanchez Galan, Javier

Sanchez Muñoz, Raul

Sanchez Vargas, Luis Alberto

Sanchez, Ana

Sanchez, Goar

Sánchez, Juan Antonio

Sánchez, Robersy

Sánchez, Teresa

Sánchez-De-Diego, Cristina

Sanchez-Infantes, David

Sancho, Jaime

Sancho-Vaello, Enea

Sandbichler, Adolf

Sandeep, Dave

Sanders, Yan

Sandjo, Louis Pergaud

Sandlers, Yana

Sandomenico, Annamaria

Sandovici, Ionel

Sandrock, Inga

Sands, Jeff M

Sandsmark, Danielle

San-Félix, Ana Rosa

Sanfilippo, Cristina

Sangadala, Sreedhara

Sangiao-Alvarellos, Susana

Sangineto, Moris

Sangiovanni, Enrico

Sangireddy, Sasikiran Reddy

Sanguedolce, Francesca

Sanguigni, Valerio

Sanij, Elaine

Sanjairaj, Vijayavenkataraman

Sanjeev, Chawla

Sanjeewa, Kalu Kapuge Asanka

Sankararaman, Senthilkumar
Sankarasubramanian, Vishwanath

Sanmartí, Raimón

Sanna, Fabrizio

Sansberro, Pedro A

Sansone, Anna

Santabarbara, Stefano

Santacroce, Luigi

Santamaria, David

Santamaría, Rafael

Santamaria, Rita

Santamaría-Martinez, Albert

Santambrogio, Paolo

Santangelo, Carmela

Santarelli, Andrea

Santarriaga, Stephanie

Santhakumar, Abishek Bommannan

Santhanam, Abirami

Santhoshkumar, Puttur

Santi, Chiara De

Santi, Claudio

Santi, Luca

Santi, Melissa

Santi, Simonetta

Santiago, James Patrick

Santino, Angelo

Santocki, Michal

Santoni-Rugiu, Eric

Santoro, Nicola

Santoro, Rosaria

Santos De Almeida, Tânia

Santos López, Jorge Arturo

Santos Vizcaíno, Edorta

Santos, Bruno A C M

Santos, Carla

Santos, Carla S.

Santos, Hugo

Santos, Jerran

Santos, Luis

Santos, Mariana

Santos, Paulo

Santos, Rosário

Santos-Coquillat, Ana

Santos-Juanes, Jorge

Santos-Ruiz, Leonor

Santovito, Gianfranco

Santucci, Annalisa

Sanz Luque, Emanuel

Sanz-González, Silvia M.

Sanzo, Antonio

Saotome, Masao

Sapkota, Kiran 
Saponara, Simona

Saponaro, Concetta

Sapone, Anna

Sapozhnikov, Alexander

Sara, Franceschelli

Sara, Zalba

Sarafidis, Charalampos S.

Sarapultsev, Alexey

Saravanan, Prathab Balaji

Sarbini, Shahrul

Sarde, Claude-Olivier

Sardeli, Chrysanthi

Sardinha Francisco, Carolina

Sardu, Celestino

Saretzki, Gabriele

Sarhan, Dhifaf

Šarić, Ankica

Sarkadi, Balázs

Sarkar, Abhijit

Sarkar, Amrita

Sarkar, Aurijit

Sarkar, Devanand

Sarkar, Dhruba

Sarkar, Sibaji

Sarkar, Susobhan

Sarkes, Deborah A.

Sárközy, Marta

Sarma, Pranjal

Sarma, Saurav

Sarmati, Loredana

Sarmento-Ribeiro, Ana Bela

Sarmiento Soto, Manuel

Sarna, Michal

Sarna, Tadeusz

Sarnataro, Daniela

Sarniguet, Alain

Sarnow, Peter

Sarnowski, Tomasz

Sarti, Alba Clara

Sartini, Davide

Sasaki, Hiroki

Sasaki, Kotaro

Sasase, Tomohiko

Sasca, Daniel

Sase, Sunetra

Sassi, Mohamed

Sasso, Ferdinando Carlo

Sastre, Leandro

Sastre-Santos, Angela

Satake, Akiko

Sathyanesan, Aaron
Satish, Lakkakula

Satitsuksanoa, Pattraporn

Sato, Akinori

Sato, Eiichi

Sato, Fuyuki

Sato, Hiromi

Sato, Keisaku

Sato, Ken-ichi

Sato, Shuzo

Sato, Takashi

Sato, Takuichi

Satoh, Akira

Satoh, Katsuya

Satoh, Tadashi

Satou, Akira

Sattar, Sampurna

Sattler, Susanne

Sattler, Wolfgang

Satué, Katiuska

Satuito, Cyril Glenn P.

Saturnino, Carmela

Saúde, Leonor

Sauder, Christian

Sauer, Daniel

Sauer, Pieter Jj

Saule, Simon

Saulle, Irma

Saurav, Kumar

Saussez, Sven

Sauzay, Chloé

Savaşan, Süreyya

Savatin, Daniel-Valentin

Savchenko, Tatyana V.

Savilahti, Harri

Savoi, Stefania

Savoia, Paola

Savopoulos, Christos

Savu, Diana

Saw, Constance L.L.

Sawada, Kazuhiko

Sawada, Koji

Sawamura, Kentaro-ichi

Sawosz, Ewa

Sawynok, Jana

Saxena, Sugandha

Sayar, Hamid

Sayner, Sarah L.

Sazonova, Margarita A.

Scacco, Salvatore

Scaglione, Kenneth Matt

Scaini, Giselli 
Scala, Angela

Scalise, Mariafrancesca

Scalschi, Loredana

Scandurra, Roberto

Scarafoni, Alessio

Scarcia, Pasquale

Scarinci, Fabio

Scarpa, Elena

Scarpellini, Emidio

Scarpellino, Giorgia

Scarselli, Marco

Scatena, Roberto

Schaaf, Gerben J.

Schachner, Adena

Schade, Dennis

Schaefer, Matthias

Schaefer, Michael

Schäfer, Katrin

Schäfer, Reinhold

Schaffer, Ari

Schaft, Niels

Schaftingen, Emile Van

Schaiquevich, Paula

Schallner, Nils

Schänzer, Anne

Scharf, Birgit

Schaub, Günter A.

Scheau, Cristian

Schedel, Michaela

Scheel-Krüger, Jørgen

Scheffel, Jörg

Scheiner, Steve

Schellino, Roberta

Schemmer, Peter

Schempp, Christoph M.

Schepisi, Giuseppe

Scherbak, Nikolai

Scherer, Günther

Schernthaner, Johann

Scherthan, Harry

Scherz-Shouval, Ruth

Scheuer, Till

Scheuring, David

Schiaffino, Stefano

Schiattarella, Antonio

Schibler, Ueli

Schicho, Rudolf

Schicker, Klaus

Schierwagen, Robert

Schiewe, Mitchel

Schiff, Elena R.
Schiffer, Lina

Schiffmann, Raphael

Schikov, Alexander

Schildberg, Frank A.

Schildgen, Oliver

Schillaci, Domenico

Schilling, Arndt Friedrich

Schindl, Rainer

Schindler, Charles W.

Schipper, Kerstin

Schippers, Angela

Schirhagl, Romana

Schirmer, Eric

Schito, Anna Maria

Schjenken, John E.

Schlachetzki, Johannes

Schlaepfer, David

Schlaepfer, Isabel $\mathrm{R}$

Schlegel, Jürgen

Schlegel, Patrick

Schlein, Christian

Schlicker, Eberhard

Schlitt, Hans

Schlothauer, Tilman

Schlunk, Frieder

Schmalhausen, E.V.

Schmid, Rafael

Schmid, Ralf

Schmidt, Boris

Schmidt, Ed

Schmidt, Jonas Damgård

Schmidt, Marcus

Schmidt, Thomas

Schmidt-Bleek, Katharina

Schmidtke, Gunter

Schmidts, Miriam

Schmiedl, Andreas

Schmitt, Emmanuelle

Schmitt, Nicole C

Schmittgen, Thomas

Schmitt-Ulms, Gerold

Schmitz, Ingo

Schmitz, Matthias

Schmitz, Thomas

Schnabelrauch, Matthias

Schneider, Anja

Schneider, Barbara

Schneider, Bohdan

Schneider, Elena K.

Schneider, Martin

Schneider, Robert 
Schneider, Simon

Schneider-Maunoury, Sylvie

Schnieke, Angelika E.

Schoenaers, Sébastjen

Schoepp-Cothenet, Barbara

Schohn, Hervé

Scholey, James

Scholz, Carsten C.

Scholze, Alexandra

Schomburg, Lutz

Schouten, Alexander

Schrader, Andrea

Schrama, David

Schramm, Alexander

Schreck, Karisa C.

Schreiber, Martin

Schrezenmeir, Juergen

Schröder, Agnes

Schröder, Christian

Schröder, Henrik Daa

Schroeder, Anna

Schroeder, Hilke

Schroer, Sibylle

Schröpfer, Susan

Schrum, Adam

Schubert, Andrea

Schubert, Frank Richard

Schubert, Mario

Schuchardt, Mirjam

Schuck, Robert N.

Schueler, Julia

Schulte, Christian

Schulte, Marvin

Schultz, David J

Schultz, Kris Ann P.

Schulz, Burkhard

Schulz, Florian

Schulz, Herbert

Schulze, Margit

Schulze-Tanzil, Gundula

Schulze-Tanzil, Gundula Gesine

Schumacher, Julia

Schumann, Canan

Schunke, Kathryn Jaques

Schuren, Frank

Schuss, Patrick

Schutt, Amy

Schutte, Stacey

Schwaab, Juliana

Schwalbe, Ruth A.

Schwaminger, Sebastian
Schwan, Stefan

Schwan, William

Schwans, Jason P.

Schwartz, Lawrence M.

Schwarz, Nicole

Schwarz, Patrick

Schwarz, Steven M.

Schwarzbacherová, Viera

Schweitzer-Stenner, Reinhard

Schweizer, Frank

Schweizer, Michael

Schwind, Sebastian

Sciacca, Michele Francesco Maria

Scialla, Stefania

Sciamanna, Giuseppe

Sciascia, Savino

Ścibisz, Iwona

Scicali, Roberto

Scichilone, Nicola

Scieglinska, Dorota

Scilabra, Simone D.

Scioli, Maria Giovanna

Sciortino, Alice

Sciortino, Maria Teresa

Scioscia, Crescenzio

Scipioni, Anita

Sciubba, Fabio

Sciumè, Giuseppe

Scornik, Fabiana S

Scorziello, Antonella

Scott, Emma

Scott, Iain

Scotti, Marcus

Scotti, Nunzia

Scotto D'Abusco, Anna

Scozzafava, Andrea

Scrano, Laura

Scribano, Daniela

Scrima, Mario

Scuderi, Caterina

Scuderi, Soraya

Scudiero, Rosaria

Scully, Erin

Scuoppo, Claudio

Scuruchi, Michele

Scuteri, Arianna

Scuto, Maria

Scutt, Charlie

Sczepanski, Jonathan

Seaby, Eleanor

Seale, Andre P. 
Seale, Lucia A.

Sebastian, Aimy

Sebastián, David

Sebastianelli, Arcangelo

Sebastiani, Federico

Sebastiani, Marco

Secci, Daniela

Secondo, Agnese

Seco-Rovira, Vicente

Sedda, Giulia

Sedic, Mirela

Sedlačík, Michal

Sedlar, Karel

Sedlářová, Michaela

See Hoe, Louise E.

Seeliger, Claudine

Seene, Teet

Seers, Christine

Sefat, Farshid

Segarra-Marti, Javier

Segatto, Marco

Seger, Rony

Segers, Vincent

Segura, Inmaculada

Segura, Miguel F

Sehlin, Dag

Seidel, Kay

Seidel, Rüdiger W.

Seidl, Maximilian

Seifert, Georg J.

Seifi, Hamed

Seixas, Ana

Sekar, Shobana

Sekatski, Serguei

Seki, Ekihiro

Sekine, Masami

Sekine, Shiori

Sekino, Yohei

Sekiya, Sachiko

Sellars, Jonathan

Selleri, Silvia

Sellner, Johann

Seluanov, Andrei

Selva-O'Callaghan, Albert

Selvarajah, Dinesh

Selzer, Michael E.

Sembratowicz, Iwona

Semenkovich, Clay F.

Semenyuk, Pavel

Semeraro, Paola

Semeril, David
Semih, Esin

Semlali, Abdelhabib

Semple, Robert

Sempruch, Cezary

Semsarilar, Mona

Sena, Armando

Senapati, Parijat

Senbonmatsu, Takaaki

Senel, Mehmet

Senger, Moritz

Sengupta, Arjun

Sengupta, Sonali

Sensini, Alberto

Senter, Peter D

Seo, Cheong Hoon

Seo, Daisuke

Seo, Goo-Young

Seo, Hak Soo

Seo, Heewon

Seo, Je Hoon

Seo, Jungwon

Seo, Sang Woo

Seo, Shigemi

Seoane-Collazo, Patricia

Seong, Ki Moon

Sequeira, Ivana R.

Serafini Fracassini, Donatella

Serba, Desalegn

Serban, Daniela Elena

Serefko, Anna

Sereno, Denis

Sergeant, Kjell

Sergeev, Yuri

Sergeeva, Marina G.

Sergeeva, Olga

Serino, Giovanna

Serio, Gabriella

Sério, Susana

Serpente, Maria

Serpersu, Engin Halit

Serra, Diana

Serra, Gregorio

Serra, Massimo

Serradji, Nawal

Serrador Peiró, Juan M.

Serrano, Antonio L.

Serrano, Aurelio

Serrano, Dolores R.

Serrano, Javier Leon

Serrano, Juan Alfonso Ayala

Serrano, María 
Serrano, Mercedes

Serrano-Aroca, Ángel

Serrano-Mislata, Antonio

Serrato, Antonio

Sertic, Sarah

Sertorio, Mathieu

Seruggia, Davide

Serve, Kinta

Servent, Denis

Servín-Garcidueñas, Luis Eduardo

Seshadri, Mukund

Šestan, Nenad

Seth, Punit P.

Sethi, Sunjay

Setiadi, Hendra

Setny, Piotr

Seto, Toshiyuki

Setzer, William N.

Seubert, John M

Seung-Taek, Lee

Ševčík, Jan

Sevcikova, Sabina

Severini, Cinzia

Severino, Beatrice

Severino, Paolo

Severson, Aaron F.

Sevigny, Mary

Sevilla, Michael

Sevostyanova, Victoria

Seydel, Tilo

Seyoum, Berhane

Sferruzzi Perri, Amanda

Sgambato, Alessandro

Sgarbossa, Antonella

Sgodda, Malte

Sgouros, Joseph

Sgrignani, Jacopo

Shabbir, Waheed

Shackelford, Julia

Shafahi, Ali

Shafer, William M.

Shafiq, Sarfraz

Shafique, Michael

Shah, Ajit

Shah, Dilip

Shah, Furqan A.

Shahbaaz, Mohd

Shahzad, Babar

Shaik, Anver Basha

Shaikh, Sibhghatulla

Shakibaei, Mehdi
Shakya, Akhilesh

Shakya, Anisha

Shamay, Yosef

Shamieh, Said

Shanbhag, Vinit

Shanmugam, Gnanendra

Shanmugam, Gobinath

Shannon, Oonagh

Shao, Fangwei

Shao, Haipeng

Shao, Lijian

Shao, Wei

Shao, Wen Hai

Shapiro, Jason W.

Shapiro, Joseph I

Sharaf, Kariem

Sharan, Shyam

Sharbeen, George

Sharif, Jafar

Sharma, Anchal

Sharma, Anket

Sharma, Anurag

Sharma, Arishya

Sharma, Bhesh Raj

Sharma, Dileep

Sharma, Geetanjali

SHARMA, HIMANSHU

Sharma, Isha

Sharma, Krishna

Sharma, Neeru

Sharma, Prasant

Sharma, Preeti

Sharma, Priyanka

Sharma, Ritin

Sharma, Sanjiv

Sharma, Shweta

Sharma, Sudha

Sharma, Sumana

Sharma, Sunil K.

Sharman, Edward H.

Sharova, Natalia

Sharshov, Kirill

Shaulsky, Evyatar

Shavandi, Amin

Shavrukov, Yuri

Shaw, Subrata

Shcharbin, Dzmitry

Shcherbakova, Larisa A.

Shcherban, Andrey B.

She, Maoyun

Sheardy, Richard 
Shearer, Aiden Eliot

Shearer, Jason

SHEATS, Katie

Shehadeh, Lina A.

Shei, Ren-Jay

Shekhova, Elena

Shekhtman, Alexander

Sheldrake, Helen

Shelenkov, Andrey

Shelke, Ganesh Vilas

Shellard, Adam

Shelomi, Matan

Shelton, Holly

Shen, Che-Kun James

Shen, Haihong

Shen, Jianqiang

Shen, Lishuang

Shen, Ming-Yi

Shen, Rong

Shen, Yang

Shen, Yi

Sheng, Kuanwei

Sheng, Yue

Sheng, Zhanwu

Shenkman, Boris $S$.

Shenolikar, Shirish

Sheppard, Hilary (Billy)

Sher, Yuh-Pyng

Sherbet, Gajanan V.

Sherman, Jonathan

Sherr, David H.

Sheshadri, Namratha

Shete, Varsha

Shetty, Shishir

Sheu, Jim

Sheu, Joen-Rong

Sheu, Shey-Shing

Sheval, Eugene

Shewan, Louise G.

Shi, Chunlin

Shi, Gongjun

Shi, Haitao

Shi, Jian

Shi, Junchao

Shi, Junfeng

Shi, Kuangyu

Shi, Lei

Shi, Wen

Shi, Xiaolei

Shiao, Chih-Chung

Shiao, Young-Ji
Shiba, Kiyotaka

Shibaev, Andrey

Shibata, Atsushi

Shibata, Hiroshi

Shibuya, Akira

Shidoji, Yoshihiro

Shieh, Jeng-jer

Shiina, Takashi

Shikov, Alexander

Shikov, Alexander N.

Shiku, Hitoshi

Shim, Jae-Hyuck

Shim, Joon W.

Shima, Seigo

Shimada, Ichio

Shimada, Yasuhito

Shimada, Yohta

Shimamura, Takeshi

Shimba, Shigeki

Shimbo, Takashi

Shimek, Joanna

Shimizu, Aneko

Shimizu, Kazuyuki

Shimizu, Shunichi

Shimizu, Takeyuki

Shimizu, Tatsuya

Shimizu, Yuji

Shimizu-Ibuka, Akiko

Shimmi, Osamu

Shimo, Tsuyoshi

Shimoda, Hiroshi

Shimojo, Masahito

Shimosawa, Tatsuo

Shimose, Shigeo

Shimura, Tatsuo

Shin, Dong-Hyun

Shin, Meong Cheol

Shin, Sehyun

Shin, Young Joo

Shinde, Aparna

Shinde, Arti V.

Shinde, Suhas

Shingu, Takashi

Shingu, Yasushige

Shinohara, Akira

Shinohara, Mitsuru

Shinohara, Shogo

Shinomiya, Nariyoshi

Shinozaki, Junichi

Shin-Yi, Marzano

Shioda, Norifumi 
Shiomi, Daisuke

Shippy, Teresa

Shirahata, Tatsuya

Shirai, Yasuhito

Shirasuna, Koumei

Shiraz, Parveen

Shirmanova, Marina V.

Shirokikh, Nikolay E.

Shirole, Nitin

Shirvani, Arash $\mathrm{H}$

Shishido, Tânia K.

Shishova, Maria F.

Shivapurkar, Narayan

Shivram, Haridha

Shiwaku, Hiroki

Shkolnik, Doron

Shmarakov, Igor

Shmatov, Mikhail

Shmidt, Eugenia

Shnyder, Steve

Shoaib, Muhammad

Shojaei, Shahla

Shorter, James

Shree, Sonal

Shridhar, Surekha

Shtam, Tatiana

Shu, Sherry

Shuh Ichi, Nishikawa

Shui, Hao-ai

Shukla, Arvind

Shukla, Pushp Sheel

Shukla, Siddharth

Shukla, Surendra

Shulman, Adrian

Shults, Nataliia V.

Shutang, Tan

Shuvaev, Anton N.

Siano, Francesco

Siatkowski, Idzi

Siaugue, Jean-Michel

Sibon, Ody C.M.

Sicari, Daria

Siciliano, Carlo

Siddappa, Byrareddy

Siddharth, Sumit

Siddharthan, Venkatraman

Siddiqi, Muhammad Zubair

Siddiqui, Imran

Siddiqui, Rafat A.

Sidelmann, Johannes Jakobsen

Sidles, Sara J.
Sidorenko, Viktoriya S.

Sidorkiewicz, Iwona

Sidoti, Antonina

Sieber, Alisa-Naomi

Siebert, Hans-Christian

Siegel, Amanda

Siegers, Gabrielle M.

Siegman, Marion J.

Siemer, Ansgar B.

Siemianowski, Oskar

Sieni, Elisabetta

Sieniawska, Elwira

Sieprawska, Apolonia

Sier, Cornelis

Sierecki, Emma

Siesto, Gabriella

Signore, Giovanni

Signore, Michele

Signorile, Anna

Signorini, Cinzia

Sikalidis, Angelos

Sikazwe, Donald

Sikora, Mariusz

Sil, Payel

Sil, Susmita R

Silachev, Denis

Silaghi, Ciprian N.

Silas Fernandes, Eto

Šiler, Branislav

Siligardi, Giuliano

Siliqi, Dritan

Siljamäki, Elina

Sillence, Dan J.

Sillerud, Laurel O.

Sillo, Fabiano

Silva, Adriana Ribeiro

Silva, Alexandre R

Silva, Catarina

Silva, Nuno A.

Silva, Nuno Helder Da Cruz Simões

Silva, Paula

Silva, Pedro Jorge

Silva, Sara

Silva, Simone S.

Silva, Susana N.

Silva, Susana Nunes

Silva-Abreu, Marcelle

Silvagno, Francesca

Silvas, Jesus A.

Silvestre, Miguel Angel

Silvestri, Brigida 
Silvestri, Cristian

Sima, Livia

Simal, Jesus

Simard, Marc

Simeoli, Raffaele

Simeone, Pasquale

Simeonova, Iva

Simes, Dina

Simioni, Carolina

Simirgiotis, Mario J.

Simitzi, Chara

Simkin, Andrew

Simko, Fedor

Simkó, Myrtill

Simlat, Magdalena

Simmen, Rosalia C.M.

Simmons, Peter A.

Simo, Ladislav

Simões, Marta Filipa

Simoes, Sandra

Simon, Istvan

Simon, István

Simon, Jorge

Simon, Michel

Simon, Thomas

Šimoník, Ondřej

Simonini, Sara

Simonsen, Anja Hviid

Simopoulos, Artemis

Simopoulou, Mara

Simova-Stoilova, Lyudmila Petrova

Simpson, A. John

Simpson, Julie E.

Simpson, Melanie Rae

Sims, Natalie

Sims-Lucas, Sunder

Sinanoglou, Vassilia

Since, Marc

Sinclair, David

Šindlerová, Lenka

Sing, Andreas

Singan, Vasanth R.

Singaraju, Aditya

Singh, Abhishek

Singh, Amit

Singh, Amrita

Singh, Anurag

Singh, Arashdeep

Singh, Ashok K.

Singh, Brijesh Kumar

Singh, Chandra
Singh, Deo

Singh, Harpal

Singh, Kamalendra

Singh, Manjit

Singh, Manjul

Singh, Naveena

Singh, Nisha

Singh, Pradeep

Singh, Puspendra P.

Singh, Ratnakar

Singh, Rupesh Kumar

Singh, Sandeep

Singh, Sarbjit

Singh, Seema

Singh, Shakti

Sinha, Amit

Sinha, Debottam

Sinha, Sangita

Sini, Maria Cristina

Sinico, Chiara

Sinjari, Bruna

Sinko, Patrick

Sinkovits, György

Sinner, Debora I.

Sinreih, Maša

Sintim, Herman

Sioofy-Khojine, Amirbabak

Siotto, Mariacristina

Sipos, Katalin

Sips, Patrick

Siracusa, Rosalba

Siramshetty, Vishal B.

Sirangelo, Ivana

Sirbu, Ovidiu

Sireci, Guido

Sirenko, Oksana

Sirko, Agnieszka

Sirotkin, Alexander

Sirtori, Cesare

Sirvent, Audrey

Sissung, Tristan M.

Sisto, Francesca

Sisu, Cristina

Sitarek, Przemysław

Sitia, Roberto

Sitzia, Clementina

Sivakumar, Mani

Sivakumar, Sushama

Sivalingam, Periyasamy

Sivanesan, Iyyakkannu

Sivarajan, Sajeevan Radha 
Sivathanu, Vivek

Siwek, Agata

Sizochenko, Natalia

Sjöberg, Bror Folke

Sjoholm, Ake

Sjöqvist, Sebastian

Sjöström, Jesper

Skała, Ewa

Skalioti, Chrysanthi

Skalska-Kamińska, Agnieszka

Skalski, Paweł

Skandalis, Spyros

Skarżyńska, Ewa

Skarzynski, Piotr Henryk

Skelding, Kathryn A.

Skellam, Elizabeth

Skelly, Deanne

Skerlavaj, Barbara

Skladany, Lubomir

Sklirou, Aimilia

Skogman, Barbro H.

Skonieczna, Magdalena

Skonieczna-Żydecka, Karolina

Skórczewska, Katarzyna

Skorokhod, Oleksii

Skotak, Maciej

Skouras, Athanasios

Skowrońska, Agnieszka

Skowrońska, Katarzyna

Skrabana, Rostislav

Škrabišová, Mária

Skretas, Georgios

Skruzny, Michal

Skrzypek, Edyta

Skrzypek, Klaudia

Skrzypski, Marek

Skuja, Sandra

Skundric, Dusanka S.

Skuratovskaia, Daria

Skurikhin, Evgenii Germanovich

Slade, Dea

Slade, Neda

Slavik, Roger

Slavokhotova, Anna

Slechta, Deborah

Śledziński, Tomasz

Sleigh, James

Sleigh, Jamie

Slim, Smaoui

Śliwa, Paweł

Śliwińska-Mossoń, Mariola
Śliwińska-Wilczewska, Sylwia

Śliwka, Jadwiga

Sloan, Erica K.

Słoczyńska, Karolina

Słomka, Artur

Slota, Jessy A.

Slotkin, R. Keith

Słowińska, Mariola

Slukvin, Igor

Sluyter, Ronald

Smaaland, Rune

Smaga, Irena

Smahel, Michal

Smalla, Karl-Heinz

Smalley, Joshua L.

Smani, Tarik

Smedler, Erik

Smertenko, Andrei

Šmída, Michal

Smietana, Michael

Śmigiel, Robert

Sminia, Peter

Smirnova, E.A

Smith Callahan, Laura A.

Smith, Andrew

Smith, Ann

Smith, Brittany L.

Smith, Caroline

Smith, G. Jason

Smith, Gaynor Ann

Smith, Jared B.

Smith, Jason

Smith, Julianne

Smith, Lachlan

Smith, Lloyd M.

Smith, Loren E.

Smith, Michael P.

Smith, Micholas

Smith, Micholas Dean

Smith, Rachel

Smith, Stephen B.

Smith, Wendy S.

Smolle, Maria Anna

Smuder, Ashley J.

Smykal, Petr

Smyrnias, Ioannis

Smyth, James

Sniadecki, Marcin

Snyder, Orman L.

Snytnikova, Olga A.

So, Jonathan 
Soares, Ana Raquel

Soares, Marcelo

Sobacchi, Cristina

Sobhani, Navid

Sobierajska, Katarzyna

Sobocanec, CSandra

Sobota, Radoslaw M.

Sobotta, Łukasz

Sobreira, Tiago

Sobrido-Cameán, Daniel

Socol, Yehoshua

Socorro, Silvia

Soddu, Silvia

Söderberg, Magnus P.

Söderberg-Naucler, Cecilia

Soderhall, Irene

Soe, Kent

Soengas, Pilar

Sofue, Tadashi

Sofy, Ahmed R.

Soggiu, Alessio

Sohal, Sukhwinder

Soica, Codruta

Sokol, Elizabeth

Sokolov, Alexey

Sokolova, Ekaterina A.

Sokolovskaya, Alisa

Solanki, Sumeet

Solano, María Emilia

Solcan, Carmen

Soldan, Samantha S.

Soldin, Steven J.

Solé Cañadas, Carla

Solé, Montserrat

Solecki, Wojciech Barnaba

Soleimani, Manoocher

Soler Valls, Ana Josefa

Solesio Torregrosa, Maria De La

Encarnacion

Solimando, Antonio G.

Solimando, Antonio Giovanni

Solingapuram Sai, Kiran Kumar

Soll, Dieter

Sollazzo, Manuela

Soloski, Mark J.

Solovieva, Anastasiya

Solovjeva, Olga N.

Solovyev, Nikolay

Somasundarama, Dinesh Babu

Somlyai, Gábor

Sommariva, Elena
Son, Ahjeong

Son, Yeonghoon

Sonah, Humira

Sone, Hideko

Song, Byeongwoon

Song, Dae Kyu

Song, Dan

Song, Hailong

Song, Jaewhan

Song, Junqi

Song, Kunhua

Song, Liujiang

Song, Lusheng

Song, Minjung

Song, Min-Suk

Song, Myoung-Chong

Song, Rui

Song, Wook

Song, Xinhua

Song, Youngsup

Song, Ziyi

Songsasen, Nucharin

Soni, Chetna

Soni, Kiran Kumar

Sonkin, Dmitriy

Sonnino, Sandro

Sonoike, Kintake

Sonowal, Himangshu

Sopper, Sieghart

Sorbi, Flavia

Sorg, Olivier

Sorge, Matteo

Soria, Federico N.

Soria-Gómez, Edgar

Soriano, Jose Miguel

Soriano, Salvador

Soriano-Arroquia, Ana

Sorice, Maurizio

Soriente, Alessandra

Sorokin, Andrey

Sorokin, Vitaly

Sorrentino, E.

Sorrentino, Francesco Saverio

Sorrentino, Nicolina Cristina

Sorriento, Daniela

Sortino, Maria Angela

Sotgia, Salvatore

Sotgiu, Stefano

Soto, Enrique

Soto-Heras, Sandra 
Soto-Hermida, Angel

Sotomayor, Camilo G.

Soto-Reyes, Ileana

Sottero, Barbara

Souazé, Frederique

Souček, P.

Soufan, Othman

Soufan, Walid

Soukup, Ales

Soukupova, Jana

Soundararajan, Meera

Soundararajan, Prabhakaran

Soundharrajan, Ilavenil

Soung, Nak-Kyun

Sousa Valente, João De

Sousa, Sérgio F.

Sousa, Sérgio Filipe

Sousa, Sílvia A.

Sousa, Sofia

Souza, Pedro

Sóvágó, Imre

Soverini, Simona

Sowa, Ireneusz

Sowndhararajan, Kandhasamy

Soyfoo, Muhammad

Spada, Alberto

Spadaro, Francesca

Spadiut, Oliver

Spagnol, Gaëlle

Spagnuolo, Rocco

Spallarossa, Andrea

Spanakis, Marios

Spang, Christoph

Spanier, Britta

Sparagna, Genevieve C.

Sparkenbaugh, Erica M.

Sparling, David

Sparrer, Konstantin M.J.

Spartalis, Eleftherios

Spartalis, Michael

Sparvoli, Francesca

Spassieva, Stefka D.

Spassov, Sashko

Specjalski, Krzysztof

Spencer, Charles T.

Spencer, Gaynor E.

Spencer, Peter S.

Spengler, Gabriella

Spengler, Ulrich

Sperti, Cosimo

Spetz, Carl Jonas Jorge
Spiegler, Juliane

Spiers, James

Spinelli, Domenico

Spinelli, Francesca Romana

Spirina, Liudmila V.

Spizzichino, Valeria

Spizzirri, U Gianfranco

Spletter, Maria

Splichal, Igor

Splichalova, Alla

Spradley, Frank T.

Spratt, Donald E.

Sprenger, Georg A.

Sprio, Andrea Elio

Sprung, Carl N.

Squadrito, Mario Leonardo

Squassina, Alessio

Squillacioti, Caterina

Squire, John

Sreedasyam, Avinash

Sreedharan, Sreejesh

Sreerama, Subramanya

Srinivas, Janaswamy

Srinivasan, Bharani

Srinivasan, Bhuvanesh

Srinivasan, Rahul

Srinivasan, Sowmya

Sripathy, Smitha

Srivastava, Akhil

Srivastava, Akriti

Srivastava, Ritesh

Srivastava, Sarika

Srivastava, Saurabh

Srivastava, Vaibhav

Srivenugopal, Kalkunte

Sroda-Pomianek, Kamila

Sroka, Zbigniew

Sroka-Bartnicka, Anna

Srotin, Igor S.

St Hilaire, Melissa A.

St John, James A.

St. Louis, Irina

Stacey, David

Stacey, Michael C.

Stachowiak, Michal K.

Stachowicz, Aneta

Stachowicz-Stencel, Teresa

Stack, Gary

Stacpoole, Peter W.

Stadlbauer-Köllner, Vanessa

Stádník, Luděk 
Stafford, James L.

Stafforst, Thorsten

Stafuzza, Nedenia Bonvino

Stäheli, Peter

Stakos, Dimitrios

Staley, Christopher

Stambuk, Nikola

Stamova, Boryana

Stana, Anca - Daniela

Stančiaková, Lucia

Stanciu, Gabriela-Dumitrita

Stanciu, Oana

Stanculeanu, Dana Lucia

Stanculescu, Ioana

Stanford, Stephanie

Stanga, Serena

Stange, Katja

Staniak, Mariola

Stanicová, Jana

Staninska-Pięta, Justyna

Stanislav, Vosolsobě

Stanisz, Beata

Stankevičius, Vaidotas

Stanković, Dalibor

Stankovic, Sanda

Stanley, Jone A.

Stanojevic, Dragana M.

Starek, Małgorzata

Stark, Amy L.

Starkuviene, Vytaute

St-Arnaud, Rene

Staruschenko, Alexander

Starz-Gaiano, Michelle

Starzyńska, Anna

Starzyński, Rafał R.

Stasi, Alessandra

Stasiak, Andrzej

Stasiak, Magdalena

Stasiak-Różańska, Lidia

Stasiolek, Mariusz

Staško, Ján

Staszewski, Ori

Stathopoulos, Constantinos

Stathopoulou, Konstantina

Stathopulos, Peter

Staudacher, Alexander

Stawikowski, Maciej J.

Stawski, Robert

Stebelova, Katarina

Stecca, Barbara

Stecco, Carla
Steel, Laura F.

Steele, John W

Steelman, Andrew

Stefanelli, Claudio

Stefani, Ambra

Stefania Longoni, Silvia

Stefania, Pucciarelli

Štefanić, Petra Peharec

Stefanidou, Maria E.

Stefanon, Bruno

Stefanovic, Ivan

Stefanowicz, Joanna

Štefulj, Jasminka

Steger, Gerhard

Steger, Gertrud

Stegner, David

Stehle, Felix

Stein, Christoph

Stein, Colleen

Stein, Matthias

Steinberg, Eliana Ruth

Steinberg, Simon

Steinle, Jena

Stella, Giulia M.

Stellavato, Antonietta

Stelzer, Franz

Stender, Stefan

Stepanenko, Aleksei A.

Stepanov, Gennady V.

Stepanova, Ekaterina

Stephen, Michael Rajesh

Stephens, Edward

Stephens, Gary

Stephenson, Mary C

Stepien, Ewa

Stepien, Karolina M.

Stępień, Łukasz

Stępnik, Maciej

Stepp, Mary Ann

Sterk, Geert Jan

Sterner, Olov

Štětina, Rudolf

Steven, Andre

Stevens, Rebecca

Stevens, Victoria L.

Stevenson, Leo

Stevenson-Lerner, Heather

Steverding, Dietmar

Steward, Robert

Stewart, Adele

Stewart, Delisha 
Stewart, James

Stewart, Jason

Stewart, Matthew

Stewart-Ornstein, Jacob

Stiburkova, Blanka

Stieber, Juliane

Stieger, Bruno

Stiles, Ashlee

Stilli, Donatella

Stillo, Francesco

Stimpfel, Martin

Stocco, Gabriele

Stocker, Reto

Stockmann, Christian

Stockmeier, Craig A.

Stoian, Dana

Stoiber, Dagmar

Stojak, Marta

Stojanović, Sanja

Stojiljkovic, Maja

Stoker, Andrew William

Stolfi, Carmine

Stolp, Helen

Stolz, Andreas

Stone, Kari L.

Stopka, Tomáš

Štorchová, Helena

Storchova, Zuzana

Storici, Francesca

Storici, Paola

Storjord, Elin

Storlazzi, Aurora

Storling, Joachim

Störmann, Sylvère

Stornaiuolo, Mariano

Stossi, Fabio

Stott, Katherine

Stotz, Henrik U.

Stout, Michael J.

Stout, Randy

Stove, Christophe P.

Stowell, Kathryn

Stoyanova, Albena

Stoychev, Georgi

Stradal, Theresia

Stragliotto, Giuseppe

Straiker, Alex

Strankowska, Justyna

Strappe, Padraig

Strasser, Richard

Straten, Per Thor
Strati, Areti

Strati, Francesco

Stratmann, Bernd

Stratton, Jo Anne

Stratton, Margaret M.

Strauss, Johannes

Strauss, Laura

Strauss, Sarah

Stravopodis, Dimitrios J.

Streckfuß-Bömeke, Katrin

Street, Maria Elisabeth

Strekalova, Tatyana

Strelnikov, Vladimir V.

Streubel, Jana

Strianese, Maria

Strich, Randy

Strick, Reiner

Strickland, Jason

Strickley, Robert

Strippoli, Raffaele

Strle, Drago

Strnad, Pavel

Strnadel, Jan

Stroffekova, Katarina

Strongin, Robert

Strudwick, Xanthe

Strugała, Paulina

Strungaru, Stefan-Adrian

Struszczyk, Marcin Henryk

Strzalkowski, Nick

Strzelecka-Kiliszek, Agnieszka

Strzemiecka, Beata

Stuart, Jeffrey

Stuckey, Ruth

Studzinski, George P.

Stuhlmann, Heidi

Stührwohldt, Nils

Stulberg, Michael

Stupka, Nicole

Stur, Elaine

Šturala, Jiří

Sturiale, Carmelo Lucio

Sturm, Brigitte N.

Sturm, Eva Maria

Sturm, Heinz

Stushnoff, Cecil

Stylli, Stanley S.

$\mathrm{Su}, \mathrm{Chia-Hao}$

$\mathrm{Su}$, Chinh Tran-To

$\mathrm{Su}$, Dan

$\mathrm{Su}$, Dong-Ming 
Su, Siyuan

$\mathrm{Su}$, Tao

Su, Xinming

$\mathrm{Su}$, Yichi

$\mathrm{Su}$, Yong

$\mathrm{Su}, \mathrm{Yu}-\mathrm{Kai}$

Subbannayya, Yashwanth

Subbian, Selvakumar

Subburaju, Sivan

Subhi, Yousif

Subramani Paranthaman, Balasubramani

Subramaniam, Nathan

Subramaniam, Srinivasa

Subramanian, Arul

Subramanian, Sowmyalakshmi

Subramanyam, Subhashree

Subrata, Sabui

Suciu, Bogdan Andrei

Sud'ina, Galina F.

Suda, Kenichi

Sudano, Mateus J.

Sudhan, Dhivya

Sudheeran, Pradeep Kumar

Suehiro, Eiichi

Suen, Der-Fen

Suenaga, Hikaru

Suetsugu, Noriyuki

Suga, Hirotaka

Sugawara, Tatsuya

Sugaya, Makoto

Sugier, Piotr

Sugii, Hideki

Sugimoto, Masahiro

Sugimura, Haruhiko

Sugisaki, Kenji

Sugishima, Masakazu

Sugiyama, Munetaka

Sugiyama, Tetsuya

Suh, Dae-yeon

Suh, Hyunsuk

Suhm, Martin A.

Suhr, Frank

Sukawa, Yasutaka

Sukharev, Sergei

Sukhov, Vladimir

Sukhovershin, Roman

Sukocheva, Olga

Sukumaran, Sunitha

Šulc, Petr

Sulek, Anna

Sulentic, Courtney
Suliman, Hagir B

Sullivan, Con

Sullivan, David

Sullivan, M. L.

Sumara, Grzegorz

Sumi, Shoichiro

Sumida, Yoshio

Sumiyshi, Tomiki

Summavielle, Teresa

Summers, Katie Lynn

Summers, Matthew

Sun, Bo

Sun, Chengwen

Sun, Chongkui

Sun, Deqiang

Sun, Fengjie

Sun, Gongqin

Sun, Guohui

Sun, Haiyan

Sun, Junjiang

Sun, Mujun

Sun, Qian

Sun, Ramon

Sun, Tian-Hu

Sun, Wenfei

Sun, Wenyue

Sun, Wujin

Sun, Xiaofei

Sun, Xiaoming

Sun, Xuepeng

Sun, Ye

Sun, Zhixiong

Sunami, Yoshiaki

Sundar, Gangadhara

Sundar, Krishna M.

Sundaram, Sivaraj Mohana

Sundaramoorthy, Pasupathi

Sundaresan, Alamelu

Sundvik, Maria

Suñer, Damian Heine

Sung, Jong Hyuk

Sung, Pil Soo

Sung, Sibum

Sung, Tzu-Cheng

Sung, Wen-Wei

Sungthong, Rungroch

Sun-Hee, Cheong

Supattapone, Surachai

Supuran, Claudiu

Šuput, Dušan

Sur, Subhayan 
Surana, Priyanka

Surapaneni, Krishna Mohan

Surapathrudu, Kanakala

Surazynski, Arkadiusz

Surber, Christian

Surendran, Kameswaran

Surmacz, Eva

Surmenev, Roman

Suryavanshi, Santosh

Suryawanshi, Vasantika

Susi, Petri

Šušor, Andrej

Sussman, Mark A.

Sustkova-Fiserova, Magdalena

Sustr, Marek

Sutter, Thomas

Sutton, Granger

Suwan, Keittisak

Suzuki, Aussie

Suzuki, Hideo

Suzuki, Hiroetsu

Suzuki, Keiko

Suzuki, Maiko

Suzuki, Masaaki

Suzuki, Masako

Suzuki, Masataka G.

Suzuki, Motofumi

Suzuki, Nobuhiro

Suzuki, Norio

Suzuki, Toru

Suzuki, Toshikazu

Suzuki, Yuichiro J.

Švandová, Eva Budisova

Švastová, Eliška

Sven, Gläsker

Svensson, Maria K.

Svetlov, Maksim

Svingen, Terje

Sviridov, Denis

Svirshchevskaya, Elena

Svoboda, Jan

Svobodová, Jana

Swami, Umang

Swamy, Ganesh

Swanson, Kenneth D.

Swartz, Steven Zachary

Swartz, Talia H.

Swarup, Ranjan

Swayne, Leigh-Anne

Sweadner, Kathleen J.

Sweetman, Martin
Swelum, Ayman A

Swenson, Nathan G.

Świątek, Piotr

Świątkiewicz, Iwona

Swiatkowska, Agata

Swida-Barteczka, Aleksandra

Swiecilo, Agata

Swiercz, Aleksandra

Świerczek, Jan

Swierczynski, Julian

Swieringa, Frauke

Świerniak, Andrzej

Świętnicki, Wiesław

Syahputra, Khairul

Sybilski, Adam J.

Syed, Farooq

Syed, Naeem

Syed, Nelofer

Syeda, Aisha

Syed-Ab-Rahman, Sharifah Farhana

Syranidou, Evdokia

Syrjanen, Stina

Syrris, Petros

Sytykiewicz, Hubert

Szabo, Andras

Szabó, András

Szabo, Aron

Szabó, Kornélia

Szabó, László

Szabo, Milan

Szabó, Renáta

Szachniuk, Marta

Szacon, Elzbieta

Szade, Krzysztof

Szafrańska, Katarzyna

Szakiel, Anna

Szalai, Gabriella

Szaleniec, Maciej

Szállási, Árpád

Szanto, Magdolna

Szaryńska, Magdalena

Szászi, Katalin

Szatkiewicz, Jin P.

Szatmari, Erzsebet Maria

Szczegielniak, Jadwiga

Szczepanek, Joanna

Szczepanowska, Karolina

Szczesny, Spencer

Szebeni, Gábor János

Széchenyi, Aleksandar

Szedlacsek, Stefan 
Szeiffova Bacova, Barbara

Szeliga, Monika

Széll, Márta

Szentesi, Péter

Szepesi, Agnes

Szepesi, Ágnes

Szepietowska, Barbara

Szerdahelyi, Emőke

Szeto, Savio Yim Tong

Szeto, Yim-Tong

Szewczuk, Myron R.

Szewczyk, Katarzyna

Szewczyk-Golec, Karolina

Szilágyi, András

Szkaradkiewicz, Andrzej

Szklarz, Grazyna

Szklarzewicz, Janusz

Szoboszlai, Norbert

Szöllősi, Attila Gábor

Szöllősi, Dániel

Szondy, Zsuzsa

Szoor, Arpad

Szopa, Aleksandra

Szóstek-Mioduchowska, Anna

Sztanke, Krzysztof

Szteyn, Kalina

Sztiller-Sikorska, Malgorzata

Sztul, Elizabeth

Szulc-Dąbrowska, Lidia

Szuts, David

Szweda, Piotr

Szwengiel, Artur

Szychlinska, Marta Anna

Szyfter, Krzysztof

Szymańki, Paweł

Szymańska, Grażyna

Szymańska, Katarzyna Patrycja

Szymborski, Tomasz

Taank, Vikas

Taanman, Jan-Willem

Tabares, Lucia

Tabary, Olivier

Tabęcka-Łonczyńska, Anna

Tabler, Tom

Tabuchi, Masashi

Tacconelli, Stefania

Tachibana, Shinjiro

Tachibanaki, Shuji

Tacke, Frank

Tada, Rui

Tada, Seiichi
Tada, Yayoi

Tadepalli, Sirimuvva

Tadié, Jean-Marc

Tadini Buoninsegni, Francesco

Tadyszak, Krzysztof

Tae-Jin, Kim

Tafani, Marco

Tagad, Harichandra

Tagang, Aluwong

Taghibiglou, Changiz

Tagliaferri, Pierosandro

Tagliatesta, Pietro

Tagliavia, Marcello

Taglietti, Angelo Maria

Tago, Kenji

Taguchi, Yoshihiro

Taha, Muhamed Kheir

Taha, Muhammad

Tahara, Hidetoshi

Tahir, Ammar

Tahrioui, Ali

Taibi, Khaled

Tailhades, Julien

Taillandier, Daniel

Taishi, Nakamura

Tajeddine, Nicolas

Taji, Teruaki

Tajiri, Kazuto

Tajiri, Naoki

Tajuelo, Javier

Tak, Eunyoung

Takač, Iztok

Takács-Vellai, Krisztina

Takada, Akihiko

Takada, Tappei

Takagi, Junichi

Takagi, Masatoshi

Takahashi, Akihisa

Takahashi, Akiko

Takahashi, Daisuke

Takahashi, Hidekazu

Takahashi, Junko

Takahashi, Ken

Takahashi, Melissa K

Takahashi, Misa

Takahashi, Shinya

Takahashi, Taku

Takahashi, Toshio

Takahashi, Yutaka

Takai, Atsushi

Takai, Kazuyuki 
Takai, Shinji

Takai, Yasushi

Takam Kamga, Paul

Takami, Akiyoshi

Takano, Hudson

Takano, Junpei

Takano, Katsura

Takano, Tomomi

Takasawa, Shin

Takashi, Ishida

Takashi, Yuichi

Takata, Keiichi

Takata, Minoru

Takatani-Nakase, Tomoka

Takaya, Junji

Takaya, Tomohide

Takayama, Kazuo

Takayama, Kenichi

Takebayashi, Shigeo

Takeda, Makio

Takeda, Mamoru

Takeda, Yoshiyu

Takemori, Hiroshi

Takemoto, Shinji

Takenaka, Mizuki

Takenaka, Nobuyuki

Takeshita, Fumitaka

Takeshita, Kazutaka

Takeuchi, Hideyuki

Takeuchi, Jun

Takeuchi, Koh

Takeuchi, Tomoharu

Takeuchi, Yasuhiro

Takhter, Ramandeep S.

Takino, Junichi

Takito, Jiro

Takizawa, Shinobu

Takuma, Kazuhiro

Tala, Joana A.

Talaat, Iman M.

Talagas, Matthieu

Talavera, Karel

Talbert, Erin

Talesa, Vincenzo Nicola

Talhada, Daniela

Tallini, Luciana R.

Talora, Claudio

Talukdar, Prabhat K.

Talukder, Poulami

Taly, Antoine

Tam, Roger Y.
Tamagawa-Mineoka, Risa

Tamagnone, Luca

Tamaki, Tetsuro

Tamasi, Gabriella

Tamayo, Esther

Tamba, Bogdan Ionel

Tambe, Mitali

Tambuwala, Murtaza

Tame, Jeremy

Tamkovich, Svetlana

Tampa, Mircea

Tamura, Koji

Tamura, Yasuhisa

Tan, Aaron C.

Tan, Bee K

Tan, Bee Kang

Tan, Daniel

Tan, Du-Xian

Tan, Howe-Siang

Tan, Joanne T.M.

Tan, Karen

Tan, Longzhi

Tan, Nguan Soon

Tan, Shyh Han

Tan, Sih Min

Tan, Yuqi

Tanabe, Hideyuki

Tanabe, Katsuyuki

Tanabe, Kenji

Tanabe, Shihori

Tanaka, Atsushi

Tanaka, Hiroyoshi Y.

Tanaka, Hiroyuki

Tanaka, Hisashi

Tanaka, Ichidai

Tanaka, Kiwamu

Tanaka, Masaru

Tanaka, Miyako

Tanaka, Naoro

Tanaka, Nobuhiro

Tanaka, Nobuyuki

Tanaka, Satoshi

Tanaka, Shigeyuki

Tanaka, Takuji

Tanaka, Toshiya

Tanaka, Yasuko

Tanaka, Yuka

Tancini, Brunella

Tanda, Enrica Teresa

Tanda, Naoko

Tang, Chih-Hsin 
Tang, Damu

Tang, Fengru

Tang, Ho Lam

Tang, Ho Man

Tang, Jing

Tang, Jingjing

Tang, Jonathan

Tang, Kai

Tang, Ming-Jer

Tang, Xiaoli

Tang, Xiaoyun

Tang, Yao

Tang, Zhihua

Tangadanchu, Vijai Kumar Reddy

Tani, Akio

Tani, Eleni

Tanic, Nikola

Tanida, Takashi

Taniguchi, Hiroaki

Tanimizu, Naoki

Tanini, Damiano

Tanini, Marco

Tanino, Karen

Taniuchi, Ichiro

Tankiewicz-Kwedlo, Anna

Tanner, Nathan

Tannock, Lisa R.

Tanonaka, Kouichi

Tanphaichitr, Nongnuj

Tansi, Felista L.

Tanti, Goutam Kumar

Tantos, Ágnes

Tao, Feng

Tao, Junyan

Tao-Cheng, Jung-Hwa

Taoka, Yousuke

Tapia, Jose A.

Tapio, Soile

Tappia, Paramjit

Tar, Krisztina

Tarakhovskaya, Elena

Tarallo, Antonietta

Tarallo, Roberta

Tarang, Shikha

Taranta-Janusz, Katarzyna

Tarantino, Giovanni

Tarantino, Umberto

Tarasova, $\mathrm{Ol}^{\prime} \mathrm{Ga} \mathrm{A}$.

Tarcea, Monica

Tarn, Woan-Yuh

Tarone, Aaron
Tarozzi, Andrea

Tarsitano, Achille

Tasca, Carla

Tash, Joseph S.

Tashima, Kimihito

Tashima, Toshihiko

Tashiro, Etsu

Tashiro, Mitsuru

Tasso, Bruno

Tasso, Roberta

Tate, Rothwelle

Tate, Shin-Ichi

Tateishi, Kensuke

Tatischeff, Irène

Tattikota, Sudhir Gopal

Tattini, Massimiliano

Tatu, Alin Laurenţiu

Taub, Mary

Taube, Joseph

Tauber, Eran

Tauber, James

Taubert, Helge

Tauriello, Daniele

Tautermann, Christofer S

Tavakoli, Javad

Tavares, Anthony

Tavares, Luciana

Taves, Matthew

Tavío, María M.

Tavolari, Simona

Tayebati, Seyed Khosrow

Tayebi, Nahid

Tayeh, Nadim

Taylan, Fulya

Taylor, Alison

Taylor, Allen

Taylor, Erika A.

Taylor, Isaiah

Taylor, Keith

Taylor, Mark S.

Taylor, Matthew

Tazawa, Hiroshi

Tchertanov, Luba

Tchetina, Elena V.

Tchoghandjian-Auphan, Aurélie

Te Velthuis, Aartjan

Teale, William

Tedeschi, Tullia

Tee, James

Teerds, Katja J.

Tegeder, Irmgard 
Teijeira, Álvaro

Teisseyre, Andrzej

Teixeira, Rita

Tejedor, Rosa M.

Telegina, Darya V.

Téletchéa, Stéphane

Tella, Sri Harsha

Tellez-Isaias, Guillermo

Tellez-Lance, Angela

Tellier, Michael

Tembrock, Luke R.

Temel, Aslihan

Tempesta, Maria

Tempfer, Herbert

Tenbrock, Klaus

Tenenbaum, Liliane

Teng, Ba-Bie

Teng, Peng

Teng, Yang D.

Teng, Yong

Tenkorang, Mavis

Tenkumo, Taichi

Tennstedt, Pierre

Tenore, Gian

Tentler, Dmitri

Teo, Jeremy

Teodorescu, Mihaela

Teotia, Arun Kumar

Teotia, Sachin

Téoulé, Evelyne

Ter Kuile, Benno

Teranishi, Katsunori

Teras, Riho

Terasaki, Michishige

Terayama, Hayato

Terenin, Ilya M.

Terent'ev, Alexei

Terentes-Printzios, Dimitrios

Terentyev, Vasily V.

Terenzi, Alessio

Terlikowska, Katarzyna

Terracciano, Daniela

Terracciano, Rosa

Terradas, Mariona

Terret, Marie-Emilie

Terrinoni, Alessandro

Teruel-Montoya, Raúl

Teruyama, Ryoichi

Terzaghi, William

Terzaghi, William Bryan

Terzi, Valeria
Terzibasi, Tozzini Eva

Terzić, Janoš

Terzuoli, Lucia

Tesarik, Jan

Teschke, Rolf

Tesfaye, Dawit

Teshima, Takanori

Tesic, Zivoslav

Tessarz, Peter

Tessem, Jeffery $S$.

Tessema, Gezahegn Girma

Testa, Ugo

Testolin, Raffaele

Tétard-Jones, Catherine

Tetens, Jens

Teti, Gabriella

Tettamanti, Gianluca

Tetz, George

Tevosian, Sergei G.

Texido Bartes, Robert

Thackray, Alana

Thaker, Youg Raj

Thakur, Basant Kumar

Thakur, Garima

Thakur, Mathew

Thakur, Shilpa

Thalamuthu, Anbupalam

Thalhammer, Anja

Thalhammer, Theresia

Thalheim, Torsten

Thalmann, Matthias

Thammina, Chandra Sekhar

Than, Nandor Gabor

Than, Tin A.

Thangavel, Chellappagounder

Thanigaimani, Shivshankar

Thankachan, Stephen

Thannickal, Thomas C.

Tharkeshwar Raghunath, Arun Kumar

Thavasyappan, Thambi

The, Erlinda

Theile, Dirk

Theiss, Carsten

Then, Cornelia

Therese, Riedemann

Théron, Laëtitia

Theus, Michelle Hedrick

Thevenod, Frank

Thewke, Douglas P.

Thiel, Cora S.

Thiel, Gerald 
Thieme, René

Thierry, Hauet

Thies, Stephan

Thirunavukkarasu, Shyamala

Thoene, Jess

Thomale, Jürgen

Thomas, Bobby

Thomas, Carole D.

Thomas, Clément

Thomas, Debby

Thomas, Julie

Thomas, Noel

Thomas, Shane

Thomas, Thresia

Thomas, Wayne

Thompson, Alexander

Thompson, Joyce

Thompson, LaDora

Thompson, Peter

Thomson, David M.

Thongprayoon, Charat

Thornton, Sophie

Thorpe, Stephen

Thounaojam, Menaka Chanu

Threlkeld, Steven

Thrivikraman, Greeshma

Thuillier, Raphaël

Thulstrup, Peter W.

Thurotte, Adrien

Thurston, John H.

Thussagunpanit, Jutiporn

Thyele, Geoffrey

Tian, Furong

Tian, Kun Viviana

Tian, Li

Tian, Shiliang

Tian, Ya-Chung

Tiao, Mao-Meng

Tiapko, Oleksandra

Tiberini, Antonio

Ticconi, Carlo

Tidow, Henning

Tien, Lu-Tai

Tiera, Marcio

Tietze, Alesia

Tiganescu, Ana

Tigyi, Gabor

Tijing, Leonard

Tikellis, Christos

Tikhanovich, Irina

Tikhenko, Natalia D.
Tikhomirova, Irina

Tikhonova, Irina

Tikkanen, Ritva

Tilbrook, Alan

Tillekeratne, L.m. Viranga

Tilli, Tatiana M

Tilocca, Bruno

Timashev, Peter

Timchenko, Tatiana

Timko, Michael

Timmerman, Kyle

Timmins, Joanna

Timofeev, Edward N.

Timonen, Juri

Timoshenko, Alexander V.

Timoshevskiy, Vladimir A.

Timpani, Cara

Timpano, Massimiliano

Timperio, Anna Maria

Timsit, Youri

Ting, Richard C. H.

Tinganelli, Walter

Tinoco, Arthur D.

Tintera, Jaroslav

Tintino, Saulo

Tiperciuc, Brindusa

Tirado-corbala, Rebecca

Tiranti, Valeria

Tirino, Virginia

Tirrò, Elena

Tischkau, Shelley A.

Tisi, Renata

Tisoncik-Go, Jennifer

Tissier, Paul Le

Titeca-Beauport, Dimitri

Tittarelli, Andrés

Titus, Mark

Titz, Bjoern

Tiwari, Rakesh K.

Tiwari, Shashikant

Tjernberg, Ivar

Toan, Sam

Tobita, Morikuni

Tocmo, Restituto

Toczylowska-Maminska, Renata

Toda, Tomohisa

Todaka, Daisuke

Todd, Lauren

Todd, Nevins W.

Todi, Sokol

Todorov, Svetoslav Dimitrov 
Togashi, Yuichi

Togawa, Atsushi

Toghraee, Davood

Tognini, Paola

Tognolini, Massimiliano

Togo, Fumiharu

Tohidnezad, Mersedeh

Toietta, Gabriele

Toillon, Robert-Alain

Tojima, Ichiro

Tokar, Erik

Tokarek, Tomasz

Tokars, Valerie L.

Tokarz, Paulina

Tokas, Theodoros

Tokmakov, Alexander A.

Tokuda, Shinsaku

Tokumaru, Yoshihisa

Tokumoto, Toshinobu

Tokunaga, Fuminori

Tokuraku, Kiyotaka

Tolba, René Hany

Tolga, Karsili

Toljan, Karlo

Tolomelli, Alessandra

Tolwinski, Nicholas

Toma, Claudio

Toma-Fukai, Sachiko

Tomasetti, Carmine

Tomasetti, Marco

Tomasetto, Catherine

Tomašovičová, Natália

Tomassetti, Antonella

Tomas-Zapico, Cristina

Tomaszewska-Zaremba, Dorota

Tomaszkiewicz, Marta

Tomatsu, Shunji

Tomaz, Cândida Teixeira

Tomei, Alice

Tomescu, Mirela Cleopatra

Tominaga, Kana

Tominaga-Wada, Rumi

Tomita, Kozo

Tomita, Shunsuke

Tomiyama, Arata

Tomkin, Gerald H

Tomkinson, Nicholas

Tommasino, Francesco

Tommasino, Massimo

Tommonaro, Giuseppina

Toms, Derek
Tomuleasa, Ciprian

Tona, Franceso

Tonazzini, Ilaria

Tondi, Donatella

Tondo, Mireia

Tong, Mancy

Tong, Mingming

Tong, qiang

Tong, Wei

Tönges, Lars

Tonhajzerova, Ingrid

Toniolo, Antonio Q.

Toniolo, Luana

Tonolo, Giancarlo

Top, Janetta

Topf, Ulrike

Topin, Jeremie

Toplak, Ivan

Tordai, Attila

Toriba, Taiyo

Toriello, Filippo

Torigoe, Kanjiro

Törn, Carina

Tornillo, Giusy

Toro, Mario Damiano

Toro, Roberta

Török, Katalin

Torra, Joel

Torralba-Cabeza, Miguel-Ángel

Torras, Juan

Torras-Ambròs, Joan

Torre, Marialuisa

Torreggiani, Massimo

Torregrosa, Ruben

Torrens, Francisco

Torres Barcelo, Clara

Torres Sangiao, Eva

Torres, Adrian Gabriel

Torres, Magdalena

Torres-Lagares, Daniel

Torres-Mendoza, Daniel

Torres-Ruiz, Raul

Torres-Sanchez, Sonia

Torsello, Barbara

Torson, Alex

Torti, Mauro

Toru, Ichiseki

Toruner, Gokce Altay

Torzewska, Agnieszka

Tósaki, Árpád

Toscani, Anita 
Toscani, Denise

Toseland, Christopher P.

Tostivint, Hervé

Totah, Rheem

Toth, Andras Jozsef

Tóth, Gergő

Tóth, Melinda Erzsébet

Tóth-Molnár, Edit

Tóthová, Lubomíra

Toto, Angelo

Totsingan, Filbert

Totta, Pierangela

Tõugu, Vello

Touriol, Christian

Toussaint, Ariane

Tovo, Pier-Angelo

Tovoli, Francesco

Tower, John

Towse, Clare-Louise

Toyoda, Masao

Toyoda, Masashi

Tozawa, Yuzuru

Tozer, Gillian

Tőzsér, József

Tozza, Stefano

Tozzi, Maria Grazia

Trabocchi, Andrea

Traiffort, Élisabeth

Traina, Giovanna

Traina-Dorge, Vicki L.

Traini, Tonino

Traka, Maria

Trammell, Samuel

Tramontano, Donatella

Tramontano, Marco

Tran, Anh Nhat

Tran, Phu

Tranbarger, Timothy John

Tranebjærg, Lisbeth

Trapani, Dario

Trapani, Ivana

Trapani, Valentina

Trapero, Isabel

Trask, Sydney

Travelli, Cristina

Travlos, Ilias

Trcek, Janja

Treder, Jadwiga

Treeck, Moritz

Trefely, Sophie

Treglia, Giorgio
Trejtnar, Frantisek

Tremblay, Jean Christophe

Tremblay, Michel

Tremblay, Nicolas

Trendelenburg, Marten

Trentini, Alessandro

Trevisan, Andrea

Triaca, Viviana

Triantafillidis, John $\mathrm{K}$

Tribulova, Narcis

Tricarico, Domenico

Trifonov, Vladimir

Trigatti, Bernardo

Trimarco, Bruno

Trinchera, Marco

Trinchese, Giovanna

Trindade, Alexandre

Trindle, Carl

Tripathi, Amit

Tripathi, Diwaker

Tripathi, Jaindra Nath

Tripathi, Jitendra Kumar

Tripathi, Manish

Tripathi, Manisha

Tripi, Gabriele

Tripodi, Pasquale

Trippel, Stephen B.

Tripp-Valdez, Miguel A.

Trisolino, Giovanni

Triunfo, Stefania

Trivanovic, Drenka

Troedsson, Mats H.T.

Trofimov, Aleksei V.

Trögl, Josef

Trohman, Richard

Troitsky, Alexey

Trojanowicz, Bogusz

Trojanowski, Damian

Trombik, Tomasz

Tromp, Angelino T.

Trompier, Doriane

Tronchere, Helene

Troncoso-Ponce, Adrian

Troppmair, Jakob

Trotsko, Nazar

Trotta, Francesco

Trubiani, Oriana

Trujillo-Rodríguez, María J.

Trullas, Ramón

Truncaitè, Lidija

Trunk, Katharina 
Trus, Ivan

Trusca, Georgeta Violeta

Truskey, George A

Trybus, Kathleen M.

Tryggestad, Jeanie

Tsaballa, Aphrodite

Tsai, Ang-Chen

Tsai, Feng-Chou

Tsai, Jaw-Shiun

Tsai, Jen-Ning

Tsai, Kun-Lin

Tsai, Kun-Ling

Tsai, Ming-Tzu

Tsai, Rongkung

Tsai, Rong-Kung

Tsai, Shiao-Wen

Tsai, Shih-Jen

Tsai, Shu-Huai

Tsai, Tsung-Hua

Tsai, Wan-Chi

Tsai, Ying-Chieh

Tsai, Yuan-Chen

Tsakmakidis, Ioannis A.

Tsakovska, Ivanka

Tsampras, Nikolaos

Tsang, Stephen H.

Tsaniklidis, Georgios

Tsapogas, Panagiotis

Tsarouhas, Konstantinos

Tsarouhas, Vasilios

Tsartsalis, Stergios

Tsatsakis, Aristidis

Tsaturyan, Andrey

Tsay, Gregory J.

Tscheliessing, Rupert

Tschernig, Thomas

Tse, Brian

Tse, William K.F.

Tseng, Fan-Gang

Tseng, Jen-Chih

Tseng, Wei-Cheng

Tseng, Yu-Hsin

Tsergouli, Katerina

Tsermpini, Evangelia Eirini

Tsiani, Evangelia

Tsiarli, Maria

Tsiartas, Panagiotis

Tsigkou, Olga

Tsika, Elpida

Tsim, Karl

Tsiolaki, Paraskevi L.
Tsirigotis, Panagiotis

Tsitsekian, Dikran

Tsitsopoulos, Parmenion P.

Tskhovrebov, Alexander G.

Tsodikov, Oleg

Tsoi, James Kit-hon

Tsolaki, Anthony G.

Tsolakis, Apostolos V.

Tsouh Fokou, Patrick Valere

Tsubaki, Masanobu

Tsuboi, Hirohito

Tsubomura, Miyoko

Tsubota, Akihito

Tsubota, Takuya

Tsuchida, Kunihiro

Tsuchida, Sachio

Tsuchiya, Hiroyuki

Tsuchiya, Masahiko

Tsuchiya, Yuichi

Tsuda, Takeshi

Tsugawa, Hitoshi

Tsui, Janice

Tsui, Kuan Hao

Tsuji, Gaku

Tsuji, Hayato

Tsuji, Hiroaki

Tsukahara, Tamotsu

Tsukahara, Toshifumi

Tsukamoto, Osamu

Tsukamoto, Tetsuo

Tsukaya, Hirokazu

Tsukita, Sachiko

Tsuruda, Toshihiro

Tsutsui, N.

Tsutsui, Shigeyuki

Tsutsumi, Hiroshi

Tsykunova, Galina

Tsytsarev, Vassiliy

$\mathrm{Tu}$, Min

$\mathrm{Tu}$, Thomas

Tubek, Stanislaw

Tucker, Matthew

Tucker, Torry A.

Tudela, Jose

Tufarelli, Vincenzo

Tukaj, Stefan

Tulotta, Claudia

Tůma, Zdeněk

Tuna, Bilge Guvenc

Tunaru, Sorin

Tundo, Silvio 
Tuomanen, Elaine

Tuominen, Hannele

Tupler, Rossella

TURANLI, Beste

Turano, Mimmo

Turano, Paola

Turato, Cristian

Turcan, Şevin

Turck, Christoph W.

Turdo, Alice

Turetta, Matteo

Turk, Dusan

Turkmen, Serhat

Türkösi, Edina

Turkowski, Kati

Turnbull, Bruce

Turnbull, John

Turner, Arlener D.

Turner, Jason D.

Turner, Jonathan

Turner, Neill

Turner, Ronald B.

Turoverov, Konstantin K.

Turowski, Tomasz W.

Turrini, Eleonora

Turula, Holly

Turunen, Mikko P.

Tuszynski, Jack A.

Tutone, Marco

Tuukkanen, Juha

Tuuminen, Tamara

Tuvi-Arad, Inbal

Twarużek, Magdalena

Twizere, Jean-Claude

Tyagi, Ashish

Tyagi, Mudit

Tyan, Yu-Chang

Tylee, Karen

Tylki-Szymańska, Anna

Tyrka, Mirosław

Tyszka-Czochara, Malgorzata

Tyunin, Alexey $\mathrm{P}$

Tyurin-Kuzmin, Pyotr

Tzanakakis, George

Tzeng, Shu-Ling

Tzima, Aliki K.

Tzur, Yonatan B.

Ubaldi, Massimo

Uchida, Shizuka

Udagawa, Nobuyuki

Uddin, Golam Mezbah
Uddin, Sardar M.Zia

Udumula, Venkata

Uebanso, Takashi

Uebe, René

Ueda, Akihiro

Ueda, Motoki

Ueda-Nakanishi, Takako

Ueffing, Marius

Ueguchi-Tanaka, Miyako

Uehara, Yoshinari

Ueno, Masaki

Ueno, Tasuku

Ueno, Yuji

Uesawa, Yoshihiro

Uetake, Hiroyuki

Uezumi, Akiyoshi

Ugolini, Francesca

Uhde-Stone, Claudia

Uhl, Eberhard

Uhm, Jae-Sun

Uhrig, R. Glen

Ukken, Fiona

Ulasov, Ilya

Uldrijan, Stjepan

Ulicna, Livia

Ulisse, Salvatore

Ulivieri, Cristina

Ullah, Imran

Ullah, Md Ashik

Ullah, Mujib

Ullmann, Enrico

Ullmann, Reinhard

Ulmer, Candice Z.

Ulrich, Benjamin

Ulrich, Craig

Um, Min Young

Um, Soyoun

Umagiliyage, Arosha Loku

Umanah, George K. E.

Umansky, Samuil

Umansky, Viktor

Umapathy, Ganesh

Umapathy, Nagavedi Siddaramappa

Umehara, Mikihisa

Umekita, Kunihiko

Umemori, Juzoh

Umemura, Akira

Umemura, Tsukuru

Unden, Gottfried

Underhill, Suzanne M.

Underwood, Mark D. 
Ungaro, Paola

Ungefroren, Hendrik

Ungureanu, Camelia

Ungurianu, Anca

Unković, Nikola

Ünlü, Cüneyt $\mathrm{H}$.

Unno, Tatsuya

Unseld, Matthias

Untergasser, Gerold

Uosaki, Hideki

Upadhya, Manoj Atmaramji

Upadhyay, Chitra

Upadhyay, Rakesh K.

Uppugunduri, Chakradhara Rao

Uppulury, Karthik

Ur Rehman, Muhammad Atiq

Uraki, Ryuta

Urbach, Valerie

Urban, Jill

Urban, Milan

Urban, Otmar

Urban, Philippe

Urbanc, Brigita

Urbanek, Konrad

Urbanek, Olga

Urbaniak, Alicja

Urbaniak, Jacek

Urbaniak, Monika

Urbano, Ana Margarida

Urbanska, Ewa M.

Urbański, Arkadiusz

Urban-Wojciuk, Zuzanna

Ureshino, Hiroshi

Urgel, Manuel Espinosa

Uribe, Juan Esteban

Urick, Mary Ellen

Urra, José Miguel

Urtenov, Makhamet

Urzi, Clara

Usama, Syed Muhammad

Ushakova, Nina A.

Ushimaru, Takashi

Usmani, Abul

Usui, Noriyoshi

Usui, Tatsuya

Usui, Yoshihiko

Utkin, Yuri N.

Uusitupa, Matti

Uversky, Vladimir

Uyeda, Taro

Uygun, Sahra
Uysal-Onganer, Pinar

Uzbekov, Rustem E.

Uzbekova, Svetlana

Uziel, Orit

V Ivanov, Alexander

Vaamonde, Carlos

Vacca, Fabrizio

Vaccarezza, Mauro

Vach, Kirstin

Vachtenheim, Jiri

Vackova, Irena

Vaclavikova, Radka

Václavíková, Radka

Vafiadaki, Elizabeth

Vaibhav, Kumar

Vaidotas, Stankevičius

Vaiman, Daniel

Vainonen, Julia

Vairetti, Mariapia

Vaiselbuh, Sarah R.

Valable, Samuel

Valadkhan, Saba

Valasi, Irene

Valcke, Roland

Valdés López, Oswaldo

Valdivielso, Pedro

Valdivieso, Henar

Vale, Carmen

Vale, Paulo

Valensin, Daniela

Valente, Joana

Valente, Sabrina

Valentina, Villari

Valentini, Federica

Valentino, Mario

Valentová, Kateřina

Valentovic, Monica

Valentukeviciene, Marina

Valenzuela, Juan Luis

Valenzuela, Stella

Valerio, Alessandra

Valiante, Salvatore

Valicherla, Guru Raghavendra

Valihrach, Lukas

Valis, Martin

Valiveti, Chaitanya

Valko, Marian

Valle, Luisa Dalla

Vallejo-Illarramendi, Ainara

Vallesi, Adriana

Vallespin, Elena 
Vallet, Sonia

Valletti, Alessio

Valli, Adrian Alejandro

Vallone, Daniela

Valotassiou, Varvara

Valsesia-Wittmann, Sandrine

Valtorta, Silvia

Valverde, Diana

Valverde, Miguel Ángel

Van Ballegooijen, Hanne

Van Bergen, Tine

Van Beuningen, Henk

Van Beusechem, Victor

Van Beusechem, Victor W.

Van Beusecum, Justin P.

Van Calsteren, Marie-Rose

Van Cruchten, Steven J.

Van Cutsem, Pierre

Van Dam, Anne-Marie

Van Dam, Peter

Van De Ven, Rieneke

Van De Vijver, Koen

Van Den Akker, Guus

Van Den Berg, Bernard

Van Den Berg, Marco Alexander

Van den Brekel, Michiel Wilhelmus Maria

Van Den Ende, Wim

Van Den Hoogen, Lucas

Van Den Meiracker, Anton H.

Van Der Does, Anne M.

Van Der Kant, Rik

Van Der Merwe, Marie

Van Der Nest, Magriet A

Van Der Pluijm, I.

Van Der Sluis, Renée M.

Van Der Vaart, Michiel

Van Der Vorst, Emiel

Van Der Wel, Myrtle J.

Van Dyke, Mark

Van Dyke, Michael

Van Eijk, Marco

Van Erp, Piet

Van Esch, Betty C. A. M.

Van Etten, Ellis S.

Van Gisbergen, Marike W.

Van Gool, Stefaan W.

Van Haute, Lindsey

Van Hook, M.J.

Van Horn, Wade

Van Houten, Ben
Van Krieken, Richard

Van Loon, Barbara

Van Meurs, Jan C.

Van Muiswinkel, Willem B.

Van Noorden, Cornelis Johannes

Forrindinis

Van Nunen, Sheryl

Van Parys, Alexander

Van Rechem, Capucine

Van Ryn, Joanne

Van Saen, Dorien

Van Sanford, David

Van Schependom, Jeroen

Van Solingen, Coen

Van Straalen, Nico M.

Van Veluw, Susanne J.

Van Vliet, Sandra

Van Wijk, Richard

Van Wuytswinkel, Olivier

VanBuren, Robert

Vancheri, Federico

Vandekerckhove, Bart

Vanden Eynde, Jean Jacques

Vandenbroeck, Koen

Vandier, Christophe

Vandooren, Jennifer

Vangala, Janakiram R.

Vangeli, Dimitra

Vanham, Guido

Vanhie, Arne

Vankayala, Raviraj

Vankova, Radomira

Vanmierlo, Tim

Vann, Willie

Vanneste, Steffen

Vannucchi, Maria-Giuliana

Vanoni, Simone

VanSaun, Michael

Vanscheeuwijck, Patrick

Vanthuyne, Nicolas

Vaquero, Monica

Váradi, Csaba

Varadwaj, Pradeep

Varallyay, Eva

Varani, Katia

Varela, Monica

Varela-Nieto, Isabel

Varese, Giovanna Cristina

Varga, Máté

Varma, Vijaykumar B.

Varna, Mariana 
Varoni, Elena Maria

Varotto, Claudio

Varra, Michela

Varrassi, Giustino

Vartiainen, Maria K.

Vas, Virag

Vasaikar, Suhas V.

Vascotto, Carlo

Vaseva, Irina I.

Vasic, Vesna

Vasilchenko, Alexey

Vasile, Francesca

Vasilescu, Catalin

Vasiljeva, Olga

Vasilyev, Alexander

Vaškelytė, Jolanta

Vasquez, Marlen

Vassallo, Josanne

Vassallo, Neville

Vassaux, Georges

Vassilaki, Niki

Vassilakos, Nikon

Vassiliadis, Themistoklis

Vassiliou, Aliki Georgia

Vasu, Srividya

Vasudevan, Sreelakshmi

Vatter, Hartmut

Vaury, Chantal

Vauthey, Eric

Vaz, Daniela C.

Vázquez-Domínguez, Evaristo

Vazquez-Hernandez, Maria

Vázquez-Tato, M. Pilar

Vázquez-Ucha, Juan C.

Vecchio, Eleonora

Vecchio, Michele

Vecerek, Branislav

Vechetti, Ivan

Vecino Cordero, Elena

Vecoli, Cecilia

Vécsei, László

Veenstra, Richard

Veerabagu, Manikandan

Veeramani, Suresh

Veeramani, Vediyappan

Veeravalli, Vijayabhaskar

Vega Alvarez, Jose Antonio

Vega-Rubín-De-Celis, Silvia

Vegeto, Elisabetta

Veglia, Gianluigi

Vegvari, Akos
Veit, Guido

Veith, Paul

Velagapudi, Ravikanth

Velasco, Roberto

Velasco-Hernández, Talía

Velayudhan, Latha

Velazquez, Miguel

Veldhuizen, Edwin

Veldhuizen, Ruud A. W.

Velegzhaninov, Ilya O.

Velena, Astrida

Veletza, Stavroula

Vélez, Marisela

Velez-Cruz, Renier

Velicky, Philipp

Velikova, Tsvetelina

Velikova, Violeta

Velisek, Libor

Velizarov, Svetlozar G.

Vellante, Federica

Vellecco, Valentina

Vemula, Harika

Venditti, Massimo

Venerando, Andrea

Venere, Monica

Venier, Paola

Venkat, Poornima

Venkatasubramanian, Sambasivan

Venkatesan, Jagadeesh K

Venkateshaiah, Sathisha U.

Venkateshwaran, Muthusubramanian

Venkateswaran, Suresh

Vennekens, Rudi

Venneri, Mary Anna

Venter, Henrietta

Ventura, Natascia

Ventura-Clapier, Renée E.

Venturin, Marco

Venturini, Carola

Venugopal, Dhamodharan

Venuti, Valentina

Vera Rojas, Jaime

Verbanac, Donatella

Verbeek, D.S.

Verbeke, Kristin

Verde, Federico

Verdeguer, Francisco

Verdijk, Robert M.

Verdoucq, Lionel

Verdú, Enrique

Veres, Szilvia 
Verestiuc, Liliana

Vergadi, Eleni

Vergani, Laura

Vergara, Alessandro

Vergely, Catherine

Verger, Stéphane

Verges, Marcel

Vergés, Marcel

Verginelli, Fabio

Verhaeghe, Johan

Verheyen, Esther

Verhoeven, Aswin

Verkhovtsev, Alexey

Verleih, Marieke

Verma, Anil

Verma, Dinesh Kumar

Verma, Rati

Verma, Suresh

Verma, Vikash

Vermaas, Joshua V.

Verna, Giulio

Vernardou, Dimitra

Vernhettes, Samantha

VERNİ, Fiammetta

Verona, Guglielmo

Veronico, Pasqua

Verpeut, Jessica

Verrecchia, Franck

Verri, Tiziano

Versacci, Paolo

Versino, Elisabetta

Verstockt, Bram

Verstraeten, Aline

Verstraeten, Inge

Verza, Simone Gasparin

Verzola, Daniela

Verzoni, Elena

Vesa, Stefan Cristian

Veselska, Oleksandra

Veskoukis, Aristidis S.

Vetere, Amedeo

Vetere, Gisella

Vetrano, Ignazio Gaspare

Vetsika, Eleni-Kyriaki

Vetvicka, Vaclav

Vezzani, Bianca

Vianello, Andrea

Viapiana, Agnieszka

Viappiani, Cristiano

Viazzi, Francesca

Vicario, Nunzio
Vicentini, Caterina

Vickstrom, Casey R

Victoni, Tatiana

Victor, Bruno

Viczian, Andras

Vidal, Luciano

Vidal, Miguel

Vidal, Rene

Vidalakis, Georgios

Vidana Mateo, Beatriz

Videau, Patrick

Videtta, Walter

Vidic, Jasmina

Vidovic, Bojana

Vidovic, Sinisa

Viehmannová, Iva

Vielba, Jesús María

Viennet, Thibault

Vietinghoff, Sibylle Von

Vieyra, Adalberto

Vieyra, Jorge Paredes

Vig, Komal

Viganò, Marco

Vigh, Laszlo

Vígh, László

Vigna, Elisa

Vignard, Julien

Vigneron, Aurelien

Vignes, Michel

Vigodner, Margarita

Vigouroux, Corinne

Viguera, Ana Rosa

Viguié, Catherine

Vijay, Geraldine Vidhya

Vijayakumar, Sarath

Vijayan, Madhuvanthi

Vijayan, Murali

Vijayavenkataraman, Sanjairaj

Vikas, Praveen

Vilasi, Silvia

Vilgelm, Anna E.

Villa, Anna

Villa, Chiara

Villa, Federica

Villafaina, Santos

Villani, Rosanna

Villanova, Valeria

Villaseñor, Alma

Vimberg, Vladimir

Viña, Dolores

Vinall, Ruth 
Viñas, Miguel

Vincenzi, Colombina

Vincenzi, Fabrizio

Vincenzo, De Leo

Vinci, Cristina

Vinciguerra, Manlio

Vindigni, Vincenzo

Vingrys, Algis

Vinjamur, Divya

Vinogradov, Evguenii

Vinuesa, Teresa

Viola, Giampietro

Virag, Jitka A.I.

Viranaicken, Wildriss

Virgilio, Antonella

Virsolvy, Anne

Virto, Mailo

Virumbrales-Muñoz, María

Visai, Livia

Visconte, Valeria

Visentin, Michele

Vishe, Mahesh

Vishnoi, Kanchan

Visser, Jenny A.

Visser, Lydia

Vistoli, Giulio

Viswanathan, Sowmya

Viswanathan, Vignesh

Vita, Federico

Vita, Roberto

Vitale, Chiara

Vitale, Fabrizio

Vitale, Giovanni

Vitek, Libor

Vitek, Michael

Vitiello, Pietro Paolo

Vitorica, Javier

Vitorino, Rui Miguel Pinheiro

Vitrac, Heidi

Vittorioso, Paola

Vives-Peris, Vicente

Vivian-Smith, Adam

Vivo, Maria

Vizoso, Francisco J.

Vizoso-Vázquez, Angel

Vlad, Cătălin Ioan

Vladimirov, Vladimir

Vladislavić, Nives

Vlahopoulos, Spiros

Vlahos, Nikos F.

Vlahovic, Tracey
Vlainić, Josipa

Vlase, Gabriela

Vlasiou, Manos

Vliagoftis, Harissios

Vlk, Martin

Vo, Dat

Vo, Tommy V

Voběrková, Stanislava

Vochita, Gabriela

Voelkers, Mirko

Voellger, Benjamin

Vogel Ciernia, Annie

Vogel, Christoph

Vogel, Michael

Vogel, Monique

Vögele, Martin

Vogiatzis, Georgios

Vogler, Georg

Vogt, Andreas

Vogt, Megan

Voisin, Maud

Voisset, Cecile

Vojta, Lea

Vokurka, Aleš

Volante, Andrea

Volcho, Konstantin

Voliani, Valerio

Volicer, Ladislav

Volkov, Vadim

Vollbrecht, Claudia

Volny, Michael

Volpato, Milene

Volpe, Elisabetta

Volta, Mattia

Von Ameln-Mayerhofer, Andreas

Von Der Haar, Tobias

Von Der Thüsen, Jan

Von Döbeln, Ulrika

Von Hänisch, Carsten

Von Heideken, Johan

Von Horsten, Hans Henning

Von Knethen, Andreas

Von Kopylow, Kathrein

Von Kügelgěn, Ivar

Von Morze, Cornelius

Von Schacky, Clemens

Vondrasek, Jiri

Voort, Peter Van Der

Voortman, Jens

Vora, Lalitkumar

Vormann, Jürgen 
Vorotelyak, Ekaterina

Voshkin, Andrey A.

Vostinaru, Oliviu

Vostrikova, Kira E.

Vourc'h, Patrick

Vrbacký, Marek

Vrecl, Milka

Vreeswijk, Maaike

Vriesekoop, Frank

Vrolijk, Misha F.

Vrzal, Radim

$\mathrm{Vu}$, Lam Dai

Vugrek, Oliver

Vujanovic, Lazar

Vujosevic, Stela

Vukasinovic, Nemanja

Vukicevic, Slobodan

Vukojevic, Katarina

Vulturar, Romana

Vuts, Jozsef

Vutukuri, Rajkumar

Vychytilova-Faltejskova, Petra

Vyhnánek, Tomáš

Vykoukal, Jody

Vylkova, Slavena

Vysotski, Eugene S.

Waberski, Dagmar

Wachten, Dagmar

Wackerlig, Judith

Wade, John

Wadzinski, Thomas

Wagler, Jörg

Wagner, Gerd

Wagner, Hanna Jeannine

Wagner, Kay Dietrich

Wahab, Fazal

Wakabayashi, Sadao

Wakamatsu, Kazumasa

Wałajtys-Rode, Elżbieta

Walcheck, Bruce

Wälchli, Sébastien

Walczak-Jędrzejowska, Renata

Waldner, Maximilian

Waldum, Helge

Wali, Jibran

Waligorski, Piotr

Waligórski, Piotr

Walker, Anthony L.

Walker, Chandler L.

Walker, Douglas G.

Walker, Jessica
Walker, Lori A.

Walker, Robert

Walker, Robert P.

Walker, Valerie

Wallace, David R.

Wallace, Graham R.

Wallace, Heather M.

Wallin, Anders

Wallin, Cecilia

Walline, Heather

Walling, Jason G.

Walne, Amanda J.

Walsh, Pamela

Walter, Ingrid

Walter, Maggie C.

Walter, Ulrich

Walters, Eugene Haydn

Walters, Judith

Wan, Dehui

Wan, Timothy Ming Hun

Wang, Zhenping

Wang, Ailin

Wang, Bang-An

Wang, Bin

Wang, Chao

Wang, Chi Chiu

Wang, Chia-Yih

Wang, Chihuei

Wang, Chrong-Reen

Wang, Chun

Wang, Chunxia

Wang, David Q.

Wang, Feng-Sheng

Wang, Fuyi

Wang, Guan

Wang, Guixue

Wang, Guliang

Wang, Guorong

Wang, Hai

Wang, Hanjun

Wang, Haolu

Wang, Hongbing

Wang, Hsiuying

Wang, Huaishan

Wang, Hui-min

Wang, Jiafu

Wang, Jie (Jane)

Wang, Jin'An

Wang, Jing

Wang, Jing-Quan

Wang, Jinkai 
Wang, Jinxiang

Wang, Jiongwei

Wang, Jiun-Lin

Wang, Joshua Jianxin

Wang, Junfeng

Wang, Kai

Wang, Kankan

Wang, Lei

Wang, Leyi

Wang, Li

Wang, Liang-Chao

Wang, Liang-Chun

Wang, Lihua

Wang, Lirong

Wang, Lu

Wang, Mingbo

Wang, Nan-kai

Wang, Pa-Chun

Wang, Peipei

Wang, Peng

Wang, Pengcheng

Wang, Peng-Hui

Wang, Qingde

Wang, Qingjun

Wang, Qinyi

Wang, Richard

Wang, Richard R.-C

Wang, Shaobin

Wang, Shenqi

Wang, Shike

Wang, Shuaishuai

Wang, Shue

Wang, Sishuo

Wang, Songlin

Wang, Tsung-Jen

Wang, Wei

Wang, Wen-Der

Wang, Wenjing

Wang, Wenxin

Wang, Xiao Qi

Wang, Xiaodong

Wang, Xiaohong

Wang, Xiaoling

Wang, Xinkun

Wang, Xue

Wang, Xuewen

Wang, Yan

Wang, Yang

Wang, Yang-Kao

Wang, Ying

Wang, Ying-Jan
Wang, Yiwei

Wang, Yongqiang

Wang, Youjin

Wang, Yu-Chieh

Wang, Yumeng

Wang, Yun

Wang, Zhanxiang

Wang, Zhaoning

Wang, Zhigang

Wang, Zilong

Wanger, Samuel

Wanigatunga, Amal Asiri

Wanke, Dierk

Wann, Angus

Wanrooij, Paulina

Ward, Brian M.

Ward, Mark

Ward, Roberta J.

Wardowska, Anna

Warnakulasuriya, Saman

Warnes, Gary

Warzecha, Zygmunt

Wase, Nishikant

Waseem, Ahmad

Washino, Satoshi

Wasilewski, Tomasz

Wasniewski, Marine

Wasson, Christopher

Wasylishen, Amanda

Waszczak, Cezary

Waszkiewicz, Ewa

Waszkiewicz, Napoleon

Watała, Cezary

Watanabe, Eiichi

Watanabe, Hiroshi

Watanabe, Masayuki

Watanabe, Mikiko

Watanabe, Mutsumi

Watanabe, Naoki

Watanabe, Satoru

Watanabe, Takafumi

Watanabe, Toshio

Watanabe, Yoichi

Watanabe, Yuichiro

Wątek, Marzena

Waters, Michael J.

Watnick, Randolph S.

Watson, Zachary

Watterson, Daniel Martin

Watts, Jennifer

Watzky, Murielle 
Wauters, Lucas

Wautier, Jean-Luc

Wawiórka, Leszek

Wawruch, Martin

Wawrzycka, Donata

Wazir, Umar

Wcisło, Piotr

Wculek, Stefanie K

Weake, Vikki M.

Weaver, Connie

Weaver, Samantha R.

Webb, Carol F.

Webb, Lauren M.

Webba Da Silva, Mateus

Webber, Caleb

Webber, Christine

Weber, Bernhard H.F.

Weber, Daniel

Weber, John

Weber, Rebekka

Webster, Jack M.

Webster, Nicholas J.G.

Wędzony, Maria

Weger, Stefan

Weghofer, Andrea

Weglarz, Wladyslaw

Wegrzyn, Alicja

Węgrzyn, Grzegorz

Wegwitz, Florian

Wehling, Peter

Wehrhan, Falk

Wei, David Changli

Wei, Gong-Hong

Wei, Kai-Che

Wei, Wei-Chyou

Wei, Wenyi

Wei, Yonglong

Wei, Yufeng

Wei, Zhishun

Weickert, Martin O.

Weidner-Glunde, Magdalena

Weigand, Julia E.

Weigmann, Benno

Weihua, Zhang (John)

Weinberg, Annelie-Martina

Weingartner, Magdalena

Weingärtner, Oliver

Weinhold, Arne

Weinstein, David A.

Weinzierl, Robert

Weisel, John W.
Weiser, Brian P.

Weiser, Douglas C.

Weiss, Johanna

Weiss, Julia

Weiss, Martin

Weißenbacher, Annemarie

Weissert, Robert

Weisz, Alessandro

Weldon, Sinead

Wellberg, Elizabeth

Wellinger, Ralf

Wellinger, Ralf-Erik

Wellington, Cheryl

Wellington, Melanie

Wells, Tim

Welsh, C. Jane

Welsh, Jane

Welsh, Joshua

Welsh, Michael

Weltens, Nathalie

Wempe, Michael

Wen, Jianjun

Wen, Wei

Wen, Xiaoyan

Wen, Yu-Ching

Wenbin, Zhan

Wende, Adam R.

Weng, Ching Feng

Weng, Xiaoyu

Wenger, Roland H

Weraduwage, Sarathi M.

Werbrouck, Stefaan P.O.

Werlé, Christophe

Werneburg, Sebastian

Werner, Craig T.

Werstuck, Geoff

Wesołowska, Olga

Wess, Tim

West, Nicholas

West, Rachel

Westhaus, Sandra

Westhoff, Mike-Andrew

Westmark, Cara

Westphal, Adrie

Wetering, Koen Van De

Weyand, Nathan

Whale, Alexandra

Wheeler, Nicole E

Whelan, Fiona

Whirl Carrillo, Michelle

Whitaker, Brian D 
Whitcomb, David

White, Christine

White, Elizabeth

White, James P.

White, John

White, Ryan M.

White, Stormi Pulver

Whitehead, Debra E.

Whiteside, Theresa L.

Whitfield, Phil

Whiting, Rebecca

Whyard, Steve

Whyte, Claire

Wibowo, David

Wickner, Reed B

Wicky Collaud, Chantal

Widemann, Emilie

Widera, Darius

Widłak, Wiesława

Wie, Myung-Bok

Wieckiewicz, Mieszko

Wieczfińska, Joanna

Wieczorek, Edyta

Wiedlocha, Antoni

Wiedman, Gregory

Wiegmans, Adrian P.

Wieland, Thomas

Wielockx, Ben

Wierichs, Richard

Wierońska, Joanna

Wierzbicka, Justyna

Wiese, Maria

Wiesner, Rudolf

Wiggs, Michael

Wight, Thomas

Wigle, Jeffrey

Wiglusz, Katarzyna

Wijaya, Ramesh

Wijtmans, Maikel

Wikel, Stephen

Wiktorska, Katarzyna

Wilczek, Piotr

Wilde, Andrew

Wilde, H. Dayton

Wildemann, Britt

Wilding, Matthew

Wiley, Luke

Wilhelm, Kerstin

Wilhelm, Therese

Wilk, Aleksandra

Wilkins, Charles L.
Wilkins, Heather

Wilkinson, Adam C.

Wilkinson, Mark D.

Wilkinson, Miles F.

Wille, Holger

Willemsen, Viola

Willett, Julia

Williams, David L.

Williams, Jessica A.

Williams, John

Williams, Stephen J.

Williams, Trevor

Willige, Björn C.

Willing, Alison E.

Willis, Rohan

Willmann, Matthew R

Willner, Paul

Wilm, Matthias

Wilmowicz, Emilia

Wilson, Anne M.

Wilson, Callum

Wilson, Debbie L.

Wilson, George D.

Wilson, Iain

Wilson, Jamie L.

Wilson, Joyce

Wilson, Michael

Wilson, Peter

Wilson, Philippe B.

Wilting, Jorg

Wilton, Steve

Wilusz, Jeff

Wimmer, Zdenek

Wimmers, Klaus

Winchester, Lee

Winckler, Thomas

Windberger, Ursula

Windhorst, Sabine

Windler, Eberhard

Windshügel, Björn

Winfree, Christopher J.

Winiarczyk, Krystyna

Winiarska-Mieczan, Anna

Winkler, Johannes

Winkler, Lars

Winn, Nathan

Winneke, Gerhard

Winocur, Ephraim

Winter, Elizabeth

Winter, Lilli

Winther, Ole 
Winum, Jean-Yves

Wiraja, Christian

Wirsching, Hans-Georg

Wirth, Markus

Wirths, Oliver

Wirz, Lisa

Wise-Draper, Trisha M.

Wiśniewski, Adam

Wisniewski, Marek

Wisniewski, Michael E.

Witczak, Carol A.

Witkin, Jeffrey M.

Witkowska, Danuta

Witkowska-Piłaszewicz, Olga

Witrick, Katherine

Witt, Stephan

Witte, Claus-Peter

Wittig, Ilka

Witting, Paul K.

Wittner, Lucia

Witwer, Kenneth

Witzany, Guenther

Witzel, Katja

Wlaz, Piotr

Wlaź, Piotr

Włodarczyk, Maciej

Włodarczyk, Marta

Włoga, Dorota

Wnuk, Agnieszka

Wnuk, Maciej

Woad, Kathryn J.

Wohlleber, Dirk

Wohlmuth, Christoph

Wójcik-Jagła, Magdalena

Wójcikowska, Barbara

Wójcik-Pszczoła, Katarzyna

Wojdyło, Aneta

Wojtas, Bartosz

Wojtunik-Kulesza, Karolina Anna

Wojtyczka, Robert

Wolach, Ofir

Wołejko, Elżbieta

Wolf, Petra

Wolff, Anette Susanne Bøe

Wolfgang, Schreiner

Wolfinger, Michael Thomas

Wolfs, Tim G.A.M.

Wolińska, Agnieszka

Wollebo, Hassen S.

Wołowiec, Stanisław

Wolski, Karol
Wolthers, Kirsten

Wołyniec, Wojciech

Wong, Chin-Chean

Wong, Ching-On

Wong, Chung

Wong, Clarence T. T.

Wong, Danny K. Y.

Wong, Hovy Ho-Wai

Wong, Lee Lee

Wong, Man-kin

Wong, Maurice

Wong, Wing

Wong, Yvette

Woo Lee, Gun

Woo, So-Youn

Wood, Jason

Wood, Scott T.

Woodbury-Smith, Marc

Woodrow, Pasqualina

Woods, Daniel F.

Worch, Remigiusz

Workman, Victoria

Wormit, Alexandra

Worrall, Margaret

Worwa, Gabriella

Woscholski, Rudiger

Wosczyna, Michael

Wöstemeyer, Johannes

Wozniak, Michal

Woźniak-Budych, Marta

Wozniak-Knopp, Gordana

Wree, Andreas

Wright, Catherine

Wrobel, Maria

Wróbel, Tomasz

Wrona, Ewa

Wrzesinski, Jan

Wrześniok, Dorota

Wrzosek, Małgorzata

$\mathrm{Wu}, \mathrm{Cen}$

Wu, Chang-Yi

Wu, Chen-Chi

$\mathrm{Wu}$, Chiao-En

Wu, Chin-Chung

$\mathrm{Wu}$, Chi-Rei

$\mathrm{Wu}, \mathrm{Chun}-\mathrm{Chi}$

$\mathrm{Wu}$, ChungChun

$\mathrm{Wu}$, Chun-Te

$\mathrm{Wu}$, Gongwei

Wu, Guan-James

Wu, Hsien-Ming 
$\mathrm{Wu}$, Hsien-Tsai

$\mathrm{Wu}$, Hsuan-Chen

$\mathrm{Wu}$, Jender

$\mathrm{Wu}$, Jian-Yong

$\mathrm{Wu}$, Jin-Ming

$\mathrm{Wu}$, John

$\mathrm{Wu}$, Jun

$\mathrm{Wu}, \mathrm{Kai}$

$\mathrm{Wu}$, Kejun

Wu, Mengxi

$\mathrm{Wu}$, Meng-Yu

$\mathrm{Wu}, \mathrm{Nan}$

$\mathrm{Wu}$, Peixin

$\mathrm{Wu}$, Ping-Hsun

$\mathrm{Wu}$, Qingyu

$\mathrm{Wu}$, Sheng-Nan

$\mathrm{Wu}, \mathrm{Shu}-\mathrm{Ju}$

$\mathrm{Wu}$, Tzong-Yuan

$\mathrm{Wu}$, Wei-Li

Wu, Wen Jeng

$\mathrm{Wu}$, Xianfang

$\mathrm{Wu}$, Ying

$\mathrm{Wu}$, Youjun

$\mathrm{Wu}, \mathrm{Yu}$-Jen

$\mathrm{Wu}$, Zeguang

Wuebbles, Ryan

Wullaert, Andy

Wylie, Benjamin J.

Xanthou, Georgina

Xaplanteri, Panagiota

Xavier, Cristina Pinto Ribeiro

Xavier, Nuno M.

Xi, Guifa

$\mathrm{Xi}_{\mathrm{i}}$, Xinping

$X_{i}$, Zhengxiong

$\mathrm{Xia}$, Jianguo

Xia, Tian

Xiao, Li

Xiao, Xia

Xiao, Xinxin

Xiao, Zhenna

Xiaobin, Huang

Xiaohan, Wang

Xiaoli, Alus

Xie, Hang

Xie, Jennifer

Xie, Lishi

Xie, Min

Xie, Mouzhe

Xie, Ruili

Xie, Ting
Xie, Wei-dong

Xie, Zhiqun

Xie, Zhongqiu

Xie, Zhong-Ru

Xilouri, Maria

$X$ in, Yin

Xing, Gengmei

Xing, Minli

Xiong, Jia

Xiong, Xin

Xodo, Luigi

$\mathrm{Xu}$, Chuan

$\mathrm{Xu}$, Chuanming

$X u$, Gege

$\mathrm{Xu}$, Hongyan

$\mathrm{Xu}$, Jian

$\mathrm{Xu}$, Jin

$\mathrm{Xu}$, Jinbin

$\mathrm{Xu}$, Junwang

Xu, Kui

$\mathrm{Xu}, \mathrm{Lu}$

$\mathrm{Xu}$, Peng

$\mathrm{Xu}$, Suowen

$\mathrm{Xu}$, Wayne

$\mathrm{Xu}$, Wenyan

$\mathrm{Xu}$, Xiaojiang

$\mathrm{Xu}$, Xingbo

$\mathrm{Xu}$, Yang

Xu, Yi-Jun

Xuan, Shouhu

Xuan, Zhenyu

Xue, Meilang

Xue, Xiang

Xue, Xingjian

Xue, Xufeng

Xynias, Ioannis N.

Yadav, Dhananjay

Yadav, Dharmendra K.

Yadav, Hemraj

Yadav, Marshleen

Yadav, Pramod

Yadav, Sharda

Yadav, Vikash Kumar

Yadav, Vinita

Yadavalli, Nataraja Sekhar

Yager, Eric

Yagi, Hiroshi

Yagi, Naoto

Yagihashi, Soroku

Yagishita, Yoko

Yahiroda, Hideki 
Yaish, Mahmoud

Yakavets, Ilya

Yakimovich, Artur

Yakovlev, Igor

Yakovlev, Vasily

Yakymovych, Ihor

Yamada, Hiroyuki

Yamada, Kenji

Yamada, Masanori

Yamada, Masumi

Yamada, Sohsuke

Yamada, Taketo

Yamada, Yohei

Yamaguchi, Akira

Yamaguchi, Kiyoshi

Yamaguchi, Naohiro

Yamaguchi, Soichiro

Yamaguchi, Yoshihiro

Yamakawa, Hiroyuki

Yamakoshi, Hiroyuki

Yamamoto, Suguru

Yamamoto, Toshiharu

Yamamoto, Yusuke

Yamamura, Hisao

Yamamura, Soichiro

Yamanaka, Kenya

Yamanaka, Ryuya

Yamane, Kyoko

Yamanishi, Kiyofumi

Yamaoka, Yusuke

Yamasaki, Masahiro

Yamashiro, Sawako

Yamashita, Akira

Yamashita, Atsushi

Yamashita, Michiaki

Yamato, Masayuki

Yamato, Takehiko

Yamauchi, Akira

Yamauchi, Yoshio

Yamawaki, Yosuke

Yamazaki, Hiroyuki

Yan, An

Yan, Chunhong

Yan, Dayun

Yan, Haidong

Yan, Hongbin (Tony)

Yan, Liang-jun

Yan, Xia

Yan, Yong-Bin

Yan, Ziying

Yanaka, Saeko
Yanda, Murali

Yáñez-Mo, María

Yang, Ai-Lun

Yang, Chang-Hao

Yang, Chao-Hsun

Yang, Chao-Yie

Yang, Chen-I

Yang, Chih-Jen

Yang, Chih-Yu

Yang, Chin-Ying

Yang, Chun Zhang

Yang, Chunhua

Yang, Chunzhang

Yang, Daiwen

Yang, Deng-Ho

Yang, Deying

Yang, Guangdong

Yang, Hung-Chi

Yang, Jee Myung

Yang, Jiale

Yang, Jiann-Jou

Yang, Jimin

Yang, Jun

Yang, Junhua

Yang, Kai-Chiang

Yang, Kevin Y.

Yang, Kuang-Yao

Yang, Kun

Yang, Lili

Yang, Lingli

Yang, MinoYang

Yang, Pingfang

Yang, Qien

Yang, Qiyuan

Yang, Rong-Cai

Yang, Sejung

Yang, Seung Hwan

Yang, Seunghwan

Yang, Shengming

Yang, Shun-Chin

Yang, Shun-Fa

Yang, Soo Jin

Yang, Sung Ho

Yang, Tzu-Sen

Yang, Weibing

Yang, Yannan

Yang, Ying-Ying

Yang, Yongkang

Yang, Young Mok

Yang, Yue

Yang, Yueh-Hsun Kevin 
Yang, Zhihong

Yann, Audic

Yanniccari, Marcos

Yano, Hajime

Yano, Masato

Yano, Shozo

Yano, Shuya

Yano, Yoshiaki

Yano, Yoshihiko

Yao, Humphrey H.

Yao, Jiayi

Yao, Qizhi Cathy

Yao, Wei

Yao, Zhenqiang

Yap, Desmond

Yap, Kiryu K.

Yapa, Asanka Sajini

Yaron, Jordan R.

Yarus, Michael J.

Yarushkina, Natalia I

Yasoda, Akihiro

Yasuda, Nina

Yasuhara, Takao

Yasuhiko, Koga

Yasuko, Manabe

Yates, Nathanael J.

Yatoh, Shigeru

Yatsunyk, Liliya

Yavin, Eylon

Yazawa, Kenjiro

Yazawa, Takashi

Yazdi, Iman

Yazdimamaghani, Mostafa

Yazlovitskaya, Eugenia M.

Ye, Haobin

Ye, Jiangchuan

Ye, Lin

Ye, Wenlei

Ye, Yu

Ye, Zhiwei

Yee, Callista Stephanie

Yee, Douglas

Yeger, Herman

Yeh, Chih-Ko

Yeh, Ching-Hui

Yeh, Chun-Nan

Yeh, Elizabeth S.

Yeh, Jun-Jun

Yeh, Kuang-Sheng

Yeh, Shu-Lan

Yeh, Te-Huei
Yehya, Nadir

Yélamos, José

Yeliseev, Alexei

Yellapu, Nanda Kumar

Yen, B. Linju

Yen, Frances T.

Yen, Kelvin

Yen, Meng-Chi

Yen, Yi-Ting

Yengo-Kahn, Aaron M.

Yeo, Seonju

Yeo, Syn

Yepuri, Nageshwar R.

Yeremenko, Nataliya

Yerushalmi, Rinat

Yeste, Marc

Yetnikoff, Leora

Yevtushenko, Dmytro

Yi, Ae-Kyung

Yi, Gibum

Yi, Sang Ah

Yi, Tao

Yi, Young-Su

Yilmaz, Mehmet

Yim, Soon-Ho

Yimlamai, Dean

Yin, Chuntao

Yin, Guowei

Yin, Kingsley

Yin, Wenqing

Yin, Yin

Ying, Hao

Ying, Le

Ying, Mingyao

Yinnon, Tamar A.

Yip, Bon Ham

Yip, Ping K.

Ylostalo, Joni H.

Yochum, Gregory S.

Yoder, Kristine

Yohda, Masafumi

Yohe, Marielle E.

Yokawa, Ken

Yokoi, Fumiaki

Yokosyo, Kengo

Yokota, Takafumi

Yoneda, Toshiyuki

Yong, Jeongsik

Yoo, Hah Young

Yoo, Jung Sun

Yoo, So Young 
Yoo, Yeong-Min

Yoo, Yung Joon

Yoon, Do-Young

Yoon, Hyeonseok

Yoon, Hyonok

Yoon, Il

Yoon, Joon-Kee

Yoon, Minjoong

Yoon, Sung-Soo

Yoong, Michael

Yorgan, Timur

Yoshida, Akihiro

Yoshida, Hiroshi

Yoshida, Kaoru

Yoshida, Katsunori

Yoshida, Kentaro

Yoshida, Manabu

Yoshida, Naofumi

Yoshida, Naoyasu

Yoshida, Shinichiro

Yoshida, Takuya

Yoshida, Toshiko

Yoshida, Yukari

Yoshie, Mikihiro

Yoshigaki, Junko

Yoshikuni, Yasuo

Yoshimatsu, Mitsuhiro

Yoshimoto, Francis K.

Yoshimura, Masami

Yoshinaga, Arata

Yoshinari, Kouichi

Yoshino, Hironori

Yoshino, Miya

Yoshioka, Ken-ichi

Yoshioka, Taiyo

Yoshioka, Wataru

Yosten, Gina

Yotova, Iveta Y.

You, Frank M.

You, Hong

You, Hye Jin

You, Hyun Ju

Younès, DELLERO

Young, Arabella

Young, Eric M.

Young, Howard A.

Young, Jason C.

Young, Jessica

Young, John S.

Young, Michael

Young-Jin, Son
Youngstrom, Daniel W.

Younis, Shady

Yousefi, Morteza

Youssef, Zahraa

Youssefian, Leila

Yruela, Inmaculada

$\mathrm{Yu}$, Cheng-Chia

$\mathrm{Yu}, \mathrm{Chia-Li}$

$\mathrm{Yu}$, Chunsong

$\mathrm{Yu}$, Fengqun

$\mathrm{Yu}$, Gang

$\mathrm{Yu}$, Hsu-Sheng

$\mathrm{Yu}$, Il-Jeoung

Yu, Jian Q.

$\mathrm{Yu}$, Jingyou

Yu, Jiujiu

Yu, Kitaoka

$\mathrm{Yu}, \mathrm{Lu}$

Yu, Meifang

$\mathrm{Yu}$, Minzhong

$\mathrm{Yu}$, Tianzheng

$\mathrm{Yu}$, Weiling

Yu, Xiang

Yu, Yanbo

Yu, Yanlei

$\mathrm{Yu}$, Yanping

$\mathrm{Yu}$, Yong

Yu, Yung-Luen

Yuan, Chiun-Jye

Yuan, Hao

Yuan, Jian

Yuan, Kuo-Ching

Yuan, Peiguo

Yuan, Shyng Shiou F.

Yuan, Xinxu

Yuan, Xuegang

Yuchun, Du

Yudina, Lyubov

Yudoh, Kazuo

Yue, John K.

Yuemeng, Jia

Yuh, Chiou-Hwa

Yui, Kunio

Yuk, Heung Joo

Yukl, Erik T.

Yumoto, Hiromichi

Yumoto, Tetsuya

Yun, Hongseok

Yun, Hyung-Mun

Yun, Jun-Won

Yung, Hong-Wa 
Yung, Ken Kin Lam

Yura, Kei

Yura, Yoshiaki

Yurchenko, Ekaterina

Yushina, Irina D.

Yuzhalin, Arseniy

Yvergnaux, Florent

Yves, Combarnous

Yvonne, Börgeling

Zaballos, Miguel

Zabetakis, Ioannis

Zabuchi, Giuliano

Zaccagnini, Germana

Zaccaroni, Annalisa

Zacchia, Miriam

Zaccolo, Manuela

Zacharias, Helena U.

Zacharias, Martin

Zachariou, Zacharias

Zachos, George

Zachut, Maya

Żaczek, Anna J.

Zádor, Ernő

Zádori, Zoltán

Zaenker, Kurt

Zafrakas, Menelaos

Zagidullin, Naufal

Zagórska, Agnieszka

Zagrebelsky, Marta

Zaharia, Catalin

Zaheer, Rahat

Zahid, Maliha

Zaia, Joseph

Zaini, Paulo

Zaiou, Mohamed

Zaiss, Dietmar

Zaitlin, David

Zaitsev, Aleksey

Zając, Mirosław

Zakany, Roza

Zakaraya, Razia

Zakharova, Lucia

Zakhartchouk, Alexander

Zaki, Md. Hasan

Zakrocka, Izabela

Zakrzewska, Malgorzata

Zalewska, Anna

Zaltariov, Mirela-Fernanda

Zaltzman, Boris

Zalubovskis, Ravis

Zalzman, Michal
Zaman, Farasat

Zamaratskaia, Galia

Zamboni, Anita

Zambrano, Byron A.

Zamfir, Carmen Lăcrămioara

Zamocky, Marcel

Żamojć, Krzysztof

Zamora-León, S. Pilar

Zamorano-Leon, José Javier

Zamostny, Petr

Zampieri, Stefania

Zamponi, Gerald W.

Zamyatina, Alla

Zamyatnin, Andrey

Zander, Mark

Zanetti, Maurizio

Zanetti, Stefania

Zangari, Maurizio

Zangemeister-Wittke, Uwe

Zanghellini, Jürgen

Zani, Bianca Maria

Zanlungo, Silvana

Zannella, Carla

Zanón-Moreno, Vicente Calixto

Zanotti, Ilaria

Zanut, Alessandra

Zapata, Agustin

Zapata, Carlos

Zapata, Fabiola

Zapico, Sara C.

Zapico, Sara Casado

Zapolski, Tomasz

Zappavigna, Silvia

Zarà, Marta

Zaraca, Francesco

Zaravinos, Apostolos

Zaremba-Czogalla, Magdalena

Zaric, Svetislav

Zarnowska, Iwona Maria

Zarnowski, Tomasz

Zarrelli, Armando

Zarza, Xavier

Zastrow, Melissa L.

Zatsepin, Timofei

Zatterale, Federica

Zaunders, John

Zavattari, Patrizia

Zavattaro, Elisa

Zavodnik, Ilya

Zavyalova, Elena G.

Zawacka-Pankau, Joanna 
Zawilska, Jolanta B.

Zawrotniak, Marcin

Zbigniew, Brzozka

Zbroch, Edyta

Zdanowicz, Magdalena

Ždralević, Maša

Zdrowowicz, Magdalena

Zdzisińska, Barbara

Żebrowska, Aleksandra

Zedník, Jiří

Zeeman, Samuel C.

Zefferino, Roberto

Zegzouti, Hicham

Zehentmayr, Franz

Zehner, Richard

Zeidi, Mahdi

Zeier, Zane

Żelaszczyk, Dorota

Zelepuga, Elena

Zelles, Tibor

Zelli, Riccardo

Zeltner, Nadja

Zeman, Michal

Zemanova, Zuzana

Zemkova, $\mathrm{H}$.

Zemmour, David

Zenaro, Elena

Zenarruzabeitia, Olatz

Zenclussen, Ana Claudia

Zeng, Huawei

Zeng, Jia

Zeng, Melody

Zeni, Olga

Zenkevich, Irog G.

Zentner, Gabriel

Zerbini, Gianpaolo

Zerdes, Ioannis

Zernii, Evgeni Yu.

Zerze, Gül

Zettler, Lawrence W.

Zgarian, Roxana Gabriela

Zhai, Kui

Zhai, Tianrui

Zhan, Wenbo

Zhang, Aiping

Zhang, Baohong

Zhang, Cankui

Zhang, Changyi

Zhang, Chi

Zhang, Dan

Zhang, Daniel Xin
Zhang, Dawei

Zhang, Deliang

Zhang, Dianbao

Zhang, Diming

Zhang, Dong

Zhang, Duo

Zhang, Ershuai

Zhang, Fei

Zhang, Ge

Zhang, Guannan

Zhang, Gui-Rong

Zhang, Guofang

Zhang, Hongqiao

Zhang, Jianhui

Zhang, Jianmin

Zhang, Jianying

Zhang, Jilei

Zhang, Jinwei

Zhang, Jixiang

Zhang, Ke

Zhang, Keqiang

Zhang, Liping

Zhang, Liqiang

Zhang, Lu-Pei

Zhang, Qiang

Zhang, Qiangzhe

Zhang, Qiong

Zhang, Qiuyang

Zhang, Quan

Zhang, Rui

Zhang, Shixiong

Zhang, Shuang

Zhang, Shuling

Zhang, Sicong

Zhang, Ting

Zhang, Weijie

Zhang, Wencai

Zhang, Wenheng

Zhang, Wenjun

Zhang, Wenli

Zhang, Wujie

Zhang, Xiang

Zhang, Xiaohui

Zhang, Xiaotao

Zhang, Xiaoyu

Zhang, Xin

Zhang, Xingmin

Zhang, Xinxing

Zhang, Xuexin

Zhang, Yi

Zhang, Yifan 
Zhang, Yin Hua

Zhang, Yixiang

Zhang, Yixuan

Zhang, Yonghong

Zhang, Yongliang

Zhang, Yumiao

Zhang, Zhanhui

Zhang, Zhen

Zhao, Baoyin

Zhao, Chao

Zhao, Chaoyang

Zhao, Chen

Zhao, Gexin

Zhao, Hongbo

Zhao, Jie Jane

Zhao, Jinping

Zhao, Linbo

Zhao, Peishen (Elva)

Zhao, Qingnan

Zhao, Xiaoai

Zhao, Yang

Zhao, Yingxin

Zhao, Yuguang

Zhao, Yunfeng

Zhao, Yuqi

Zhao, Zhenwen

Zhdanova, Irina V.

Zhelankin, Andrey V.

Zheleva-Dimitrova, Dimitrina

Zheng, Wenwei

Zheng, Yi

Zheng, Yun-Wen

Zheng, Zhong

Zhong, Haizhen Andrew

Zhong, Jinshun

Zhong, Wei-Zhu

Zhong, Xiaobo

Zhong, Zhaohua

Zhou, Bangjun

Zhou, Beiyan

Zhou, Carol L. Ecale

Zhou, Chengji

Zhou, Chi

Zhou, Dingying

Zhou, Dong

Zhou, Hai-bing

Zhou, Hao

Zhou, Jia

Zhou, Jiajing

Zhou, Jian

Zhou, Jichun
Zhou, Jing-Jiang

Zhou, Jingying

Zhou, Lei

Zhou, Linli

Zhou, Man

Zhou, Mei

Zhou, Min

Zhou, Phoebe

Zhou, Qimin

Zhou, Rong

Zhou, Shan

Zhou, Shuanhu

Zhou, Shuntai

Zhou, Tianhao

Zhou, Weijie

Zhou, Wenchao

Zhou, Yiqun

Zhou, Zhidong

Zhou, Zhijun

Zhouravleva, Galina A.

Zhu, Bing

Zhu, Cheng

Zhu, Chris

Zhu, Dandan

Zhu, Hua

$\mathrm{Zhu}$, Jie

Zhu, Jingen

Zhu, Jinsheng

Zhu, Junjie

Zhu, Li (Licky)

Zhu, Lin

Zhu, Luchang

Zhu, Michael X.

Zhu, Qian-Hao

Zhu, Qing

Zhu, Qiuyu Martin

Zhu, Shiwei

Zhu, Tianyu

Zhu, Wei

Zhu, Wufu

$\mathrm{Zhu}, \mathrm{Yu}$

Zhu, Zhou

Zhu, Zihua

Zhu, Ziwen

Zhuang, Xiaoxuan

ZhuGe, Ronghua

Zhukov, Igor

Zhuo, Chunliu

Zhuo, Zhu

Zia, Kashif

Ziaee, Ahmad 
Žiarovská, Jana

Zidar, Nina

Žídek, Lukáš

Zidovec Lepej, Snjezana

Ziegler-Graff, Véronique

Zieleniak, Andrzej

Zielenkiewicz, Piotr

Zielonka, Stefan

Ziemann, Mark

Ziemnicka, Katarzyna

Zieniuk, Bartłomiej

Zienkiewicz, Agnieszka

Zienkiewicz, Krzysztof

Zientara-Rytter, Katarzyna

Zieseniss, Anke

Ziganshina, Ayrat

Zigo, Michal

Zilli, Thomas

Zilliox, Michael J.

Zimmer, Jacques

Zimmer, Klaus Peter

Zimmer, Marc

Zimmer, Sebastian

Zimmer-Bensch, Geraldine

Zimmerlin, Ludovic

Zimmermann, Sabine Dagmar

Zimmermannová, Olga

Zimowska, Małgorzata

Zimta, Alina-Andreea

Zingg, Jean-Marc

Zironi, Isabella

Zisapel, Nava

Zitman-Gal, Tali

Zitta, Sabine

Zivcak, Marek

Zloh, Mire

Zmora, Pawel

Zoete, Vincent

Zofall, Martin

Zoghbi, María Elena

Zoidis, Evangelos

Żok, Tomasz

Żołądek, Teresa

Zoladz, Jerzy A.

Žoldák, Gabriel

Żołek, Teresa
Zoli, Angelo

Zollino, Marcella

Zollner, Gernot

Żołnowska, Beata

Zolotukhin, Igor A.

Zondler, Lisa

Zoratti, Mario

Zorec, Robert

Zorena, Katarzyna

Zorov, Dmitry B

Zorzan, Simone

Zosky, Graham

Zottini, Michela

Zou, Chunbin

Zou, Junjie

Zou, Wei

Zou, Xueqing

Zou, Zhongwei

Zovkic, Iva

Zsurka, Gabor

Zubarev, Andrey

Zubareva, Ekaterina V

Zuba-Surma, Ewa

Zubiaga, Ana

Zubieta, Chloe

Zucconi, Laura

Zuffo, Michela

Zugaza, José Luis

Zujovic, Zoran

Zuna, Jan

Žunar, Bojan

Zuñiga, Sonia

Zúñiga-Hernández, Jessica

Zunke, Friederike

Zuo, Li

Županič, Anže

Zurawa-Janicka, Dorota

Żurawek, Magdalena

Żurawska-Płaksej, Ewa

Żurek, Grzegorz

Zurita, Mario

Zuryn, Steven

Zwacka, Ralf

Zweigerdt, Robert

Zyromska, Angieszka

Żyżelewicz, Dorota 